\title{
Dense-core vesicle maturation at the Golgi-endosomal interface in Caenorhabditis elegans
}

\author{
PhD Thesis
}

in partial fulfilment of the requirements

for the degree "Doctor rerum naturalium (Dr. rer. nat.)“

in the Molecular Biology Program

at the Georg August University Göttingen,

Faculty of Biology

Submitted by

Mandy Hannemann

Born in

Lauchhammer, Germany 
I hereby declare that the $\mathrm{PhD}$ thesis entitled, "Dense-core vesicle maturation at the Golgi-endosomal interface in Caenorhabditis elegans", was written independently and with no other sources and aids than quoted.

Göttingen, $19^{\text {th }}$ March, 2012

Mandy Hannemann

Thesis Committee Members:

Dr. Stefan Eimer

Prof. Dr. Reinhard Jahn

Prof. Dr. Nils Brose
European Neuroscience Institute Göttingen

Max Planck Institute for Biophysical Chemistry

Max Planck Institute for Experimental Medicine 


\section{Table of Contents}

Table of Contents

List of Figures

List of Tables

xi

Abbreviations

xii

Acknowledgement

xiv

\section{Abstract}

II. General Introduction

II.1 Characteristics of neuropeptides 3

II.2 Coexistence of neuropeptides and neurotransmitters in neurons 4

II.3 Mechanisms of SSV and LDCV exocytosis 5

II.4 Mode of action of neuropeptides 6

II.5 Dense-core vesicles (DCVs) biogenesis $\quad 7$

II.5.1 Lipids necessary for vesicle budding at the TGN 10

II.5.2 Granins and its role in aggregation within DCVs 10

II.6 Models for DCV formation $\quad 11$

$\begin{array}{ll}\text { II.7 Regulation of DCV biogenesis } & 14\end{array}$

II.8 Role of RAB-2 and its effector RIC-19 in DCV maturation
in C. elegans

II.9 The family of small Rab GTPases $\quad 18$

II.9.1 The conserved protein structure of Rab GTPases 19

II.9.2 Regulation of Rab GTPase activity 22

II.9.3 Function of Rab GTPases in vesicular transport 23

II.9.4 Specific functions of Rab GTPases in neurons $\quad 26$

II.10 Studying DCV function in C. elegans 27

\section{Chapter 1: Regulation of the small GTPase RAB-2 during dense-core vesicle maturation}

\section{Specific Introduction}

III.1. The Rab GAP family: TBC-domain containing proteins 32

III.2. Mode of action of Rab GAPs by a dual-finger mechanism 33

III.3. Functions of Rab GAPs $\quad 34$

III.4. Regulation of GAPs $\quad 35$

III.5. TBC-domain containing proteins in C. elegans 36

III.6. Aims of this study $\quad 40$ 


\section{Results}

IV.1 tbc-8 mutants display DCV maturation defects

IV.2 TBC-8 and RAB-2 are involved in the same genetic pathway

IV.3 TBC-8 is not involved in neuropeptide processing

IV.4. tbc-8 mutants show no morphological defects in motoneurons and no SV defects

IV.5. $t b c-8$ codes for an evolutionarily conserved Rab GAP specifically expressed in neurons

IV.6. TBC-8 shows no defects in postendocytic trafficking and is not required for degradation of apoptotic cell corpses

IV.7. TBC-8 localizes to the Golgi-endosomal interface

IV.8. TBC-8 full length and fragments of TBC-8 are not soluble when expressed in E. coli strains or insect cells

IV.9. TBC-8 is a putative RAB-2 GAP

V.1. TBC-8 is a putative neuron specific RAB-2 GAP

V.2. TBC-8 is conserved throughout evolution

V.3. TBC-8 regulates DCV maturation

V.4. Unlike unc-108/rab-2 mutants, tbc-8 deletion mutant worms display no movement defects

\section{Chapter 2: Role of a RAB-2 effector complex during dense-core vesicle maturation}

VI.1 Role of RAB-2 and its effector RIC-19 in DCV maturation in C. elegans

VI.2. Aims of this study

VII.1. TBC-8 interacts with the RAB-2 effector RIC-19/ICA69

VII.2. The novel factor, RUND-1, involved in DCV function

VII.3. RUND-1 interacts with RAB-2 in a yeast two-hybrid analysis 91

VII.4. RUND-1 interacts with the putative RAB-2 GAP, TBC-8 93

VII.5. RUND-1 interacts with the RAB-2 effector, RIC-19 96

VII.6. CCCP-1 is likely to be another RAB-2 effector important for DCV trafficking

VIII.1. RUND-1 and CCCP-1 are novel effectors of the small GTPase RAB-2 for DCV maturation in C. elegans 
VIII.2. The function of different protein domains during DCV maturation

VIII.3. Regulation of the putative RAB-2 GAP, TBC-8, during DCV maturation 106

VIII.4. The RAB-2 effector complex might be highly dynamic 107

VIII.5. The role of the RAB-2 effector complex during DCV maturation 108

\section{Chapter 3: Role of the retrograde transport pathway in dense-core vesicle maturation}

IX. Specific Introduction

IX.1. Role of tethering factors in intracellular transport 113

IX.2. Multi-subunit tethering complexes $\quad 115$

IX.3. The GARP complex in retrograde trafficking 118

IX.4. The role of the GARP subunit, Vps51, in C. elegans 120

IX.5. Aims of this study 120

X. Results

X.1. The GARP complex is involved in DCV trafficking 122

X.2. GARP subunits interact with individual RAB GTPases in Y2H 126

X.3. The GARP subunit VPS-51 interacts with RAB-2 effectors, RIC-19 and RUND-1 as well as with the putative RAB-2 GAP, TBC-8 128

X.4. The GARP subunit VPS-51 and RUND-1 act in the same pathway 129

XI.1. Function of the retrograde transport during DCV maturation

XI.2. Specific role of VPS-51 in DCV maturation

XI.3. An extended model for DCV maturation

XIII.2 Molecular biological methods

XIII.2.1 Maintenance of E. coli

XIII.2.2 Preparation of chemical competent E. coli 139

XIII.2.3 Molecular cloning 140

XIII.2.3.1 Polymerase Chain Reaction (PCR) 140

XIII.2.3.2 Digestion of DNA 140

XIII.2.3.3 Agarose gel electrophoresis $\quad 140$

XIII.2.3.4 Ligation 141

XIII.2.3.5 Chemical transformation of E. coli 141

XIII.3 Protein biochemical methods 141

XIII.3.1 Expression and solubility test of recombinant proteins 141 
XIII.3.2 SDS PAGE and Western blot analysis 142

XIII.4 C. elegans based methods 143

XIII.4.1 Maintenance of C. elegans strains 143

XIII.4.2 Freezing and recovery of C. elegans stocks 143

XIII.4.3 Synchronization or decontamination of C. elegans

(egg preparation) 144

XIII.4.4 Crossing of C. elegans 144

XIII.4.5 Generation of transgenic C. elegans worm lines via microinjection 144

XIII.4.6 Single worm PCR / Worm PCR 145

XIII.4.7 Movement assay 145

XIII.4.8 Co-immunoprecipitation of proteins from C. elegans extracts 145

XIII.4.9 RNA interference (RNAi) by feeding 146

XIII.4.10 Microscopic analysis of C. elegans 147

XIII.4.11 High pressure freezing/Freeze substitution/
Electron microscopy

XIII.4.12 Texas red-conjugated BSA endocytosis assay 149

XIII.4.13 ssGFP endocytosis assay 149

XIII.5 Tissue culture based methods $\quad 150$

XIII.5.1 Maintenance of cell lines $\quad 150$

XIII.5.2 Transfection of cell lines 150

XIII.5.3 Co-immunoprecipitation of proteins from transfected HEK293 cells 150

XIII.6 Yeast based methods $\quad 151$

$\begin{array}{ll}\text { XIII.6.1 Maintenance of yeast strains } & 151\end{array}$

XIII.6.2 Yeast tansformation 151

XIII.6.3 Yeast-Two Hybrid system 151

XIII.7 Bioinformatics 152

$\begin{array}{lr}\text { XIV. Bibliography } & 153\end{array}$

$\begin{array}{lr}\text { XV. Appendix } & 174\end{array}$

$\begin{array}{ll}\text { XV.1 Materials and Reagents } & 174\end{array}$

XV.1.1 Laboratory equipment $\quad 174$

XV.1.2 Chemicals 175

XV.1.3 Kits/Enzymes/Reagents 176

XV.1.4 Antibodies 177

XV.2 Strains and cell lines 177

$\begin{array}{ll}\text { XV.3 Oligonucleotides } & 180\end{array}$

$\begin{array}{lll}\text { XV.4 Plasmids } & 182\end{array}$

$\begin{array}{ll}\text { XV.5 Media } & 187\end{array}$

$\begin{array}{ll}\text { XV.6 Buffers and Solutions } & 188\end{array}$

$\begin{array}{lll}\text { XV.7 } & \text { Supplementary Figures } & 193\end{array}$

$\begin{array}{ll}\text { Curriculum Vitae } & 195\end{array}$

$\begin{array}{ll}\text { List of Publication } & 197\end{array}$ 


\section{List of Figures}

Figure II.1. Subcellular localization of neuropeptides at central synapses in mouse and rat by double-immunogold labeling of CGRP/SP

Figure II.2. Simplified representation of possible modulations by neuropeptides at synapses

Figure II.3. Steps involved in DCV biogenesis and maturation

Figure II.4. Protein domain structure of CgA and SgIII

Figure II.5. Key steps for neuropeptide sorting mediated by SgIII and cholesterol-rich membrane at the TGN, Step 1, Step 2, Step 3

Figure II.6. Regulation of DCV biogenesis

Figure II.7. The small GTPase RAB-2 is involved in the retention of cargo during DCV maturation

Figure II.8. The intracellular localization of Rab GTPases

Figure II.9. General protein structure of Rab GTPases

Figure II.10. General protein structure of Rab GTPases

Figure II.11. Prenylation of the C-terminus of Rab proteins leads to reversible membrane localization

Figure II.12. The Rab cycle

Figure II.13. Rab GTPase functions in vesicle trafficking

Figure II.14. Cholinergic motoneurons of the ventral nerve cord (VNC) project to the dorsal nerve cord (DNC) in C. elegans

Figure II.15. DCVs can be labeled by expressing the proneuropeptide NLP-21 fused to VENUS in cholinergic motoneurons in C. elegans

Figure II.16. NLP-21-VENUS assay to detect DCV trafficking and secretion defects 
Figure III.1. The Rab cycle

Figure III.2. Protein structure of the transition state of the TBC-GAP, Gyp1p, in complex with Rab33-GDP-AlF 3

Figure III.3. Mechanisms to regulate RAB GAP activity or localization

Figure III.4. Molecular dendrogram of human (Homo sapiens) and nematode (C. elegans) TBC proteins

Figure III.5. Structure of TBC-domain containing proteins in C. elegans

Figure IV.1. Analysis of mutants of TBC-domain containing GAPs in C. elegans for DCV trafficking defects of the NLP-21-VENUS marker

Figure IV.2. $\quad t b c-8(t m 3802)$ mutants show decreased NLP-21-derived VENUS levels in the dorsal nerve cord and coelomocytes

Figure IV.3. Downregulation of $t b c-8$ expression leads to decreased fluorescence levels of VENUS derived from NLP-21 in the dorsal nerve cord 43

Figure IV.4. $\quad t b c-8(t m 3802)$ showed decreased NLP-21-derived VENUS levels 44

Figure IV.5. $\quad t b c-8(t m 3802)$ mutants show similar DCV trafficking defects as unc-108/rab-2(n501) mutants

Figure IV.6. The VENUS-tag derived from other neuropeptides than NLP-21 was also lost in $t b c-8(t m 3802)$ mutants

Figure IV.7. IDA-1-GFP trafficking is not affected in $t b c-8(t m 3802)$ mutants

Figure IV.8. TBC-8 is involved in the same pathway as UNC-108/RAB-2

Figure IV.9. Loss of the soluble VENUS cargo in $t b c-8(t m 3802)$ mutants is rescued by overexpression of constitutively active, GTP-bound RAB-5 (Q78L)

Figure IV.10. TBC-8 is not involved in the generation of active NLP-21 neuropeptides

Figure IV.11. $t b c-8(t m 3802)$ mutants do not display movement defects 
Figure IV.12. Unlike egl-3(gk238); unc-108/rab-2(n501) mutants, double mutants of egl-3(gk238) and tbc-8(tm3802) display no additive movement defects

Figure IV.13. $t b c-8(t m 3802)$ mutants display no defects in synaptic vesicle trafficking

Figure IV.14. $t b c-8(t m 3802)$ mutants do not show morphological changes

Figure IV.15. $t b c-8(t m 3802)$ mutants display no defects in synaptic vesicle trafficking and localization

Figure IV.16. Gene structure of $t b c-8$

Figure IV.17. TBC-8 is evolutionarily conserved

Figure IV.18. $t b c-8$ is expressed in the nervous system of $C$. elegans

Figure IV.19. Steady-state endocytosis of coelomocytes in $t b c-8$ mutants is unaffected

Figure IV.20. The fluid-phase endocytosis is unaffected in $t b c-8$ mutants

Figure IV.21. Fluid-phase endocytosis of Texas red-BSA was unaltered in tbc-8(tm3802) mutants

Figure IV.22. $t b c-8(t m 3802)$ mutants do not show defects in degradation of apoptotic cell corpses in the germ line

Figure IV.23. TBC-8 localizes to the Golgi-endosomal interface

Figure IV.24. Representation of TBC-8 fragments that were used to test expression and solubility in E. coli

Figure IV.25. TBC-8 fragments are not soluble when expressed in E. coli

Figure IV.26. TBC-8 is a putative RAB-2 GAP

Figure IV.27. TBC-8 contains a conserved catalytic arginine finger within the TBC-domain

Figure IV.28. TBC-8 is a putative RAB-2 GAP

Figure IV.29. Interactions of TBC-8 with RAB-2 and RAB-19 are GTP-dependent 
Figure IV.30. rab-19(ok1845) mutants do not display defects in DCV trafficking of the NLP-21-VENUS marker in the DNC

Figure V.1. Schematic representation of neuronal DCV maturation in C. elegans

Figure VI.1. RAB-2 and its effector, RIC-19 are involved in DCV maturation

Figure VII.1. The RAB-2 effector, RIC-19/ICA69 genetically interacts with the putative RAB-2 GAP, TBC-8

Figure VII.2. TBC-8 and RIC-19 co-localize in neurons

Figure VII.3. The RAB-2 effector, RIC-19, and the putative RAB-2 GAP, TBC-8, interact in yeast two-hybrid analysis and co-immunoprecipitation

Figure VII.4. Domain mapping of TBC-8 for interaction with RIC-19

Figure VII.5. Schematic representation of the gene rund-1 and the protein domain structure of RUND-1

Figure VII.6. rund-1 mutants display movement defects

Figure VII.7. RUND-1 is involved in DCV trafficking

Figure VII.8. RUND-1 specifically interacts with constitutively active, GTP-bound RAB-2 in $\mathrm{Y} 2 \mathrm{H}$

Figure VII.9. RUND-1 might interact with RAB-2 via its RUN-domain

Figure VII.10. RUND-1 does not interact with other small GTPases in a $\mathrm{Y} 2 \mathrm{H}$ analysis

Figure VII.11. RUND-1 interacts with the putative RAB-2 GAP, TBC-8

Figure VII.12. Domain mapping of TBC-8 for interaction with RUND-1 
Figure VII.13. RUND-1 interacts with the RAB-2 effector, RIC-19 in a co-IP experiment

Figure VII.14. Schematic representation of detected interactions of proteins involved in DCV trafficking

Figure VII.15. Double mutant analysis of TBC-8 and the RAB-2 effectors, RIC-19 and RUND-1 using the NLP-21-VENUS DCV assay

Figure VII.16. Schematic representation of protein domains detected in CCCP-1

Figure VII.17. CCCP-1 interacts with RAB-2 in a GTP-dependent manner

Figure VII.18. CCCP-1 does not interact with TBC-8 nor to the RAB-2 effectors, RIC-19 and RUND-1 in a Y2H analysis

Figure VIII.1. Schematic representation of protein domains found in DCV factors, TBC-8, CCCP-1, RUND-1 and RIC-19

Figure VIII.2. The C. elegans genome encodes for six RUN-domain containing proteins

Figure VIII.3. Schematic representation of a novel RAB-2 effector required for DCV maturation in C. elegans neurons

Figure IX.1. Transport of cargo-loaded vesicles between organelles occurs in four distinct steps

Figure IX.2. Classification of tethering factors based on their structure

Figure IX.3. The CATCHR subgroup of multi-subunit tethering complexes is structurally related

Figure IX.4. Different transport pathways are mediated by multi-subunit tethering complexes

Figure IX.5. The GARP complex interacts with small GTPases and SNARE proteins during retrograde trafficking 
$\begin{array}{lll}\text { Figure X.1. GARP mutants display movement defects } & 123\end{array}$

Figure X.2. GARP mutants show defects in DCV trafficking in the DNC 124

Figure X.3. GARP mutants show less fluorescence of endocytosed VENUS in the coelomocytes

Figure X.4. GARP mutants display no obvious defects in synaptic vesicle trafficking

Figure X.5. The GARP subunits VPS-51 and VPS-53 interact with different RAB GTPases in a Y2H screen

Figure X.6. The GARP subunit VPS-51 interacts with TBC-8, RIC-19 and RUND-1 in $\mathrm{Y} 2 \mathrm{H}$ and in co-IP experiments

Figure X.7. VPS-51 interacts with the RUN-domain of TBC-8 in Y2H and in co-IP experiments

Figure X.8. VPS-51 and RUND-1 act in the same pathway during DCV trafficking, whereas VPS-51 and TBC-8 do not

Figure XI.1. Graphic representation of neuronal DCV maturation in C. elegans 136

Figure XIII.1. RNAi feeding vector L4440

Figure XV.1. Protein sequence alignment of TBC-8 with its orthologs SGSM1 (H. sapiens) and CG32506-PC (D. melanogaster)

Figure XV.2. TBC-8 does not interact with RAP proteins in a yeast two-hybrid analysis 


\section{List of Tables}

Table III.1. List of C. elegans tbc genes and available mutant alleles

Table IV.1. Different constructs used for solubility test of recombinant TBC-8

Table IV.2. Expression and solubility test of recombinant TBC-8 fragments used in insect cells

Table XIII.1: Different constructs used for expression and solubility test of recombinant TBC-8

Table XV.1. Laboratory equipment used in this study

Table XV.2. Chemicals used in this study

Table XV.3. Enzymes and kits used in this study

Table XV.4. Antibodies used in this study

Table XV.5. Strains and cell lines used in this study

Table XV.6. C. elegans strains used in this study

Table XV.7. Transgenic arrays used in this assay

Table XV.8. DNA sequences of primers used in this study

Table XV.9. Deletion alleles with respective worm PCR primers used in this study

Table XV.10. Plasmids used in this study 


\section{Abbreviations}

aa

AD

AMPK

AP-1

Arf

BAR

$\mathrm{BD}$

bp

CAPS

CATCHR

$\mathrm{CC}$

$\mathrm{CCV}$

$\mathrm{CgA} / \mathrm{B}$

CGRP

COG

CORVET

CPE

DAG

(L)DCV

$\mathrm{ddH}_{2} \mathrm{O}$

DENN

DIC

DNC

Ds11

EEA-1

ER

FLP

GAP

GARP

GDF

GDI

GDP

GEF

GFP

GGA

GLUT4

GPCR

GRAM

GST

GTP

HEK293
Amino acid

Gal4p DNA activation domain fusion

AMP-activated protein kinase

Adapter protein-1

ADP-ribosylation factor

Bin/Amphiphysin/Rvs

Gal4p DNA binding domain fusion

Base pairs

$\mathrm{Ca}^{2+}$-dependent activator protein for secretion

Complex associated with tethering containing helical rods

Coiled coil

Clathrin coated vesicle

Chromogranin A/B

Calcitonin gene-related peptide complex

Conserved oligomeric

Class C core vacuole/endosome tethering complex

Carboxypeptidase E

Diacylglycerol

(Large) Dense core vesicle

Double distilled water

Differentially expressed normal versus neoplastic

Differential interference contrast

Dorsal nerve cord

Dependence on SLY1-20

Early endosomal antigen-1

Endoplasmic reticulum

FMRFamide like peptides

GTPase activating protein

Golgi associated retrograde protein complex

GDI dissociation factor

GDP dissociation inhibitor

Guanosine diphosphate

Guanine nucleotide exchange factors

Green fluorescent protein

Golgi associated, $\gamma$ ear containing, ADP ribosylation factor binding protein

Glucose transporter 4

G-protein coupled receptor

Glucosyltransferases, Rab-like GTPase activators and Myotubularins

Glutathione-S-transferase

Guanosine triphosphate

Human Embryonic Kidney 293 


\begin{tabular}{|c|c|}
\hline His & Histidine \\
\hline HOPS & Homotypic vacuole protein sorting complex \\
\hline HPF-EM & High pressure freeze-electron microscopy \\
\hline IA-2 & Insulinoma-associated protein 2 \\
\hline IC & ER-Golgi intermediate compartment \\
\hline iDCV & Immature dense core vesicle \\
\hline INS & Insulin-like neuropeptide \\
\hline IP3 & Inositol $(1,4,5)$ trisphosphate \\
\hline IPTG & Isopropyl $\beta$-D-thiogalactopyranoside \\
\hline LB & Luria Bertani \\
\hline MALDI TOF & Matrix-assisted laser desorption/ionization \\
\hline MANNS & Mannosidase II \\
\hline $\mathrm{mDCV}$ & Mature dense core vesicle \\
\hline MTC & Multi-subunit tethering complexes \\
\hline NGM & Nematode growth medium \\
\hline NLP & Neuropeptide like proteins \\
\hline NMJ & Neuro-muscular junction \\
\hline OD & Optical density \\
\hline PA & Phosphatidic acid \\
\hline PC1-3 & Proprotein convertase $1-3$ \\
\hline $\mathrm{PH}$ & Pleckstrin homology \\
\hline $\mathrm{P}_{\mathrm{i}}$ & Inorganic phosphate \\
\hline PTB & Polypyrimidine-tract binding protein / Phosphotyrosine-binding \\
\hline $\mathrm{Rab}$ & Ras genes from rat brain \\
\hline RHOD & Rhodanese homology domain \\
\hline RT & Room temperature \\
\hline RUN & RPIP8, UNC-14, NESCA \\
\hline SgII-IV & Secretogranin II-IV \\
\hline SH3 & Src homology domain 3 \\
\hline SNAREs & Soluble N-ethylmaleimide-sensitive factor attachment protein receptors \\
\hline SP & Substance P \\
\hline STYKc & Protein kinase domain of undetermined specificity \\
\hline$(\mathrm{S}) \mathrm{SV}$ & (Small clear) Synaptic vesicle \\
\hline $\mathrm{TBC}$ & Tre-2/Cdc16/Bub2 \\
\hline $\mathrm{TF}$ & Tethering factor \\
\hline TGN & Trans-Golgi network \\
\hline TLDc & TBC and LysM domain-containing \\
\hline $\mathrm{TM}$ & Transmembrane domain \\
\hline TR-BSA & Texas-red conjugated to bovine serum albumin \\
\hline TRAPP & Transport protein particle complex \\
\hline $\mathrm{VNC}$ & Ventral nerve cord \\
\hline Vps & Vacuolar protein sorting \\
\hline WT & Wild type \\
\hline $\mathrm{Y} 2 \mathrm{H}$ & Yeast two-hybrid \\
\hline
\end{tabular}




\section{Acknowledgement}

First of all I would like to thank my supervisor Stefan Eimer for sharing his time and knowledge with me. I was happy to obtain such an exciting research topic for my $\mathrm{PhD}$. I definitely learned for life during the last three years.

Secondly I would also like to thank my thesis committee members Prof. Reinhard Jahn and Prof. Nils Brose for their interest, advices and guidance over the last years. I am especially thankful to Prof. Reinhard Jahn for his great advice how to improve a certain method. Without this help my project would not have been gone so far. I am also very grateful to Prof. Nils Brose for his personal advices for me.

I am thankful to Dr. Steffen Burkhardt and Kerstin Grüniger from the Molecular Biology program coordination office for their endless support during my PhD years. In this aspect I would also like to thank Christiane Becker and Dr. Synnöve Beckh from the administration and coordination office at the European Neuroscience Institute (ENI) for all the administrative work and support. Many thanks to the ENI/UMG for continuous financial support during my $\mathrm{PhD}$ years.

I would like to express my appreciation for all former and present members of the lab, especially to Nora, Christian, Maike, Ling, Janny and Christoph for their support, team spirit and friendship. Working with you guys was a great experience. In this regard I would like to especially thank Dr. Marija Sumakovic for her guidance and help in the beginning of the project and Dr. Nikhil Sasidharan for just being a terrific guy! Nikhil, thank you for your friendship and your great supervision during the last years. It was a pleasure to work with you and to share thoughts about Germany, India and just life! You know whenever you need a senior scientist in your lab, I am your man! Stay just the way you are! 
Furthermore, I appreciate the helpful technical assistance provided by Katrin and Sabine. I would like to thank Ali and Matthias for awesome IT-support and Magda and Frank for providing practical help when needed.

Now I would like to thank people who provided support, results, discussion and advices during my $\mathrm{PhD}$ and therefore, pushed my projects forward. I thank Dr. Jan Hegermann for electron microscopy experiments. I am thankful to Heiko Röhse for his support and sharing his knowledge about confocal microscopy. Special thanks to Dr. John Chua for his advices for co-immunoprecipitation experiments and sharing reagents. I am also grateful to Dr. Michael Ailion for discussions and sharing results and strains. Many thanks to Prof. Henning Urlaub and his team for analyzing my samples by mass spectrometry. I am also thankful to the team of the protein expression and purification core facility at the EMBL, Heidelberg for their continuous work on protein purification. Special thanks to Prof. Janet Richmond for critical discussions on my project. I would like to acknowledge Lena Kutscher and Katharina Lindner for the help during their lab rotations. It was a pleasure to supervise you.

I would like to thank all my friends from my former periods of life for their support and fantastic distraction from work. Special thanks to Lenka and Sebastian for living with me. I am particular thankful to Dr. Peleg Shahaf for the great mensa-time and "for getting on my nerves whenever you could do so"! I loved to kick your backside in bowling and table tennis. I am thankful to my buddies Christian, Alex and Tilo for the funny daily email contact. I love you guys and miss you so much! I am especially thankful to Romy, Ines, Kathrin and Mandy for being my best friends. Thank you for always having an open ear for me!

Last but not least, I would not have survived the last years without the love and continuous support from Peer and my family (especially my parents). Thank you for your advices, encouragement and faith in me. I love your pride for me. Thank you Peer for your unbelievable patience and making every weekend more beautiful. Thank you so much for all the driven kilometers. 


\section{Dedicated to my grandfather}




\section{Abstract}

Fast synaptic transmission is mediated by the triggered release of neurotransmitters from synaptic vesicles (SVs). To regulate synaptic transmission and neuronal activity, neurons also release neuropeptides and hormones from dense-core vesicles (DCVs). While SVs can be recycled, DCVs have to be newly synthesized in the cell body after release. DCVs are believed to be generated at the trans-Golgi network (TGN) as immature DCVs, which subsequently undergo a maturation process through clathrin-mediated membrane remodeling events. This maturation process is required for efficient processing of neuropeptides within DCVs and removal of factors that would otherwise interfere with DCV release. It has been shown that only mature DCVs are able to undergo stimulus-dependent exocytosis.

Previously, we showed that the small Rab GTPase, RAB-2, and its effector, RIC19/ICA69, are involved in neuronal DCV maturation in Caenorhabditis elegans. In rab-2 mutants, specific cargo is lost from maturing DCVs and mis-sorted into the endosomallysosomal degradation route. This cargo loss could be prevented by blocking endosomal delivery. This suggested that RAB-2 is involved in retention of DCV components during the sorting process at the Golgi-endosomal interface. To understand how RAB-2 activity is regulated at the Golgi, we screened for RAB-2 specific GTPase activating proteins (GAPs). We identified a potential RAB-2 GAP, TBC-8, which is exclusively expressed in neurons, and when depleted, shows similar DCV maturation defects as rab-2 mutants. We could demonstrate that RAB-2 binds to its putative GAP, TBC-8.

Furthermore, we found novel factors involved in DCV maturation, RUND-1 and CCCP-1. Interestingly, both RAB-2 effectors, RUND-1 and RIC-19, interacted with the 
negative regulator of RAB-2, the GAP TBC-8, in binding studies. Therefore, RAB-2 might recruit its own GAP via its effector complex, which would enhance its deactivation. This suggests that the regulation of RAB-2 at the Golgi is highly-dynamic during DCV maturation. This negative feedback loop might represent a novel mechanism to regulate Rab function.

Moreover, we have shown for the first time that retrograde trafficking is also required during DCV maturation by analyzing the involvement of the multi-subunit Golgi-associated retrograde protein (GARP) tethering complex. We propose that active RAB-2 might facilitate the reception of retrograde trafficking vesicles delivered back to the maturing DCV compartment at the Golgi-endosomal interface.

All these findings indicate that DCV maturation is a highly-regulated process that relies on the cooperation of various proteins, such as Rab GTPases and large multisubunit tethering complexes. 


\section{General Introduction}

\section{II.1 Characteristics of neuropeptides}

Like low-molecular-weight neurotransmitters, neuropeptides are neuronal signaling molecules. They are small polypeptides consisting of 3-100 amino acids and are about 50 times larger than classical, low-molecular-weight neurotransmitters. Therefore, neuropeptides contain usually more recognition sites for receptors than neurotransmitters (Salio et al, 2006). As a consequence, they show a higher binding affinity and selectivity for their own receptors compared to neurotransmitters resulting in downstream biological effects, even at lower concentrations (Salio et al, 2006). Previously, it has been shown that neuropeptides have a high half-life in the extracellular space suggesting that their activity is longer lasting than that of classical neurotransmitters (Mens et al, 1983).

The most important task of neuropeptides is to play an essential role in direct neuronal communication and in modulating chemical neurotransmission (Salio et al, 2006). Furthermore, neuropeptides contribute in morphological plasticity in the nervous system (Theodosis et al, 1986). In addition to regulating neuronal functions in close proximity to the site of release, neuropeptides are also particularly important for regulating overall behaviors of an organism, such as growth, reproduction and satiety (Hagan \& Niswender, 2012; Kumar, 2011; Messinis et al, 2010) and also act during inflammation (Hopkins \& Rothwell, 1995; Rothwell \& Hopkins, 1995). 


\section{II.2 Coexistence of neuropeptides and neurotransmitters in neurons}

Coexistence of two or more transmitters is a common feature of neurons (Hokfelt, 1991; Lundberg, 1996; Merighi, 2002). It has been shown that different neuropeptides can coexist with each other or together with other neurotransmitters. In general, neurons produce a combination of one (or more) low-molecular-weight transmitter(s) and one (or more) neuropeptide(s), whereby the classical neurotransmitter is generally believed to be the main fast-acting messenger (Salio et al, 2006).

Due to the discovery of coexistence, two classes of vesicles have been reported, small clear synaptic vesicles (SSVs) for classical transmitters and large dense-core vesicles (LDCVs) for neuropeptides (Fried et al, 1985; Zhu et al, 1986). In some cases LDCVs also contain neurotransmitters, whereby peptides have never been detected within SSVs (Pelletier et al, 1981) (Figure II.1). Furthermore, it has been shown that a neuron that expresses two (or more) types of neuropeptides, stored this mixture within the same LDCVs and did not selectively package them into different LDCV subpopulations [reviewed in (Salio et al, 2006)] (Figure II.1).

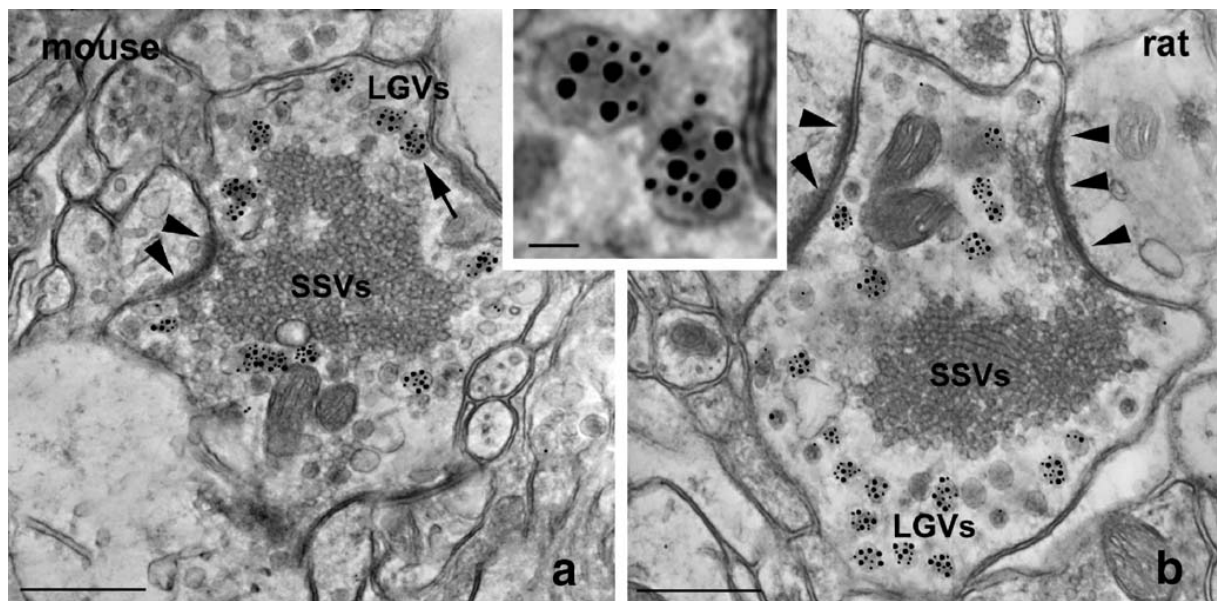

Figure II.1. Subcellular localization of neuropeptides at central synapses in mouse (a) and rat (b) by double-immunogold labeling of CGRP/SP. A glomerulus is shown that contain both SSVs and LDCVs, whereby only in LDCVs both neuropeptides were detected (double-labeling of large and small gold particles). SSVs were unlabeled. Furthermore, all LDCVs were never seen to cluster at synaptic densities (arrowheads), away from SSVs. Insert: Magnification of the costorage of CGRP (large 20-nm gold) and SP (small 10-nm gold) within the two LDCVs indicated by the arrow in (a). CGRP: calcitonin gene-related peptide, SP: substance P. Bars represent 500 $\mathrm{nm}$ (insert $50 \mathrm{~nm}$ ). Source: picture taken from (Salio et al, 2006), Figure 2, (C) Springer-Verlag 2006, with kind permission from Springer Science and Business Media. 


\section{II.3 Mechanisms of SSV and LDCV exocytosis}

This coexistence of LDCVs filled with neuropeptides and SSV containing neurotransmitters in a single neuron is a general characteristic of central and peripheral neurons (Hokfelt, 1991; Lundberg, 1996; Merighi, 2002). The release of both, neurotransmitters and neuropeptides, from one neuron allows fast $(2-5 \mathrm{~ms})$ and slow (100-500 ms) synaptic communication, respectively (Salio et al, 2006). SSVs are abundant in neurons, clustering close to the plasma membrane. LDCVs are less frequently observed at synapses and show a spatially distribution throughout the synapse (Bruns \& Jahn, 1995) (Figure II.1). Whereas a certain amount of SSVs are docked to the pre-synaptic density, known as the readily releasable pool, LDCVs are usually located distant from the pre-synaptic membrane (Salio et al, 2006) (Figure II.1). Interestingly, it was observed that LDCVs can be released at plasma membranes without any synaptic specialization (Buma, 1988; De Camilli \& Jahn, 1990; Karhunen et al, 2001; Zhu et al, 1986). In general, vesicle release is triggered by an intracellular increase of $\mathrm{Ca}^{2+}$ concentration, whereby a focal increase in $\mathrm{Ca}^{2+}$ at the synaptic density releases SSVs and diffuse elevation of $\mathrm{Ca}^{2+}$ inside the synaptic terminal leads to release of LDCVs (Verhage et al, 1991).

Two different mechanisms of transmitter release were described for SSV and LDCV exocytosis (Artalejo et al, 1998; Harata et al, 2001; Tsuboi \& Rutter, 2003). Beside the classical exocytosis event resulting in the complete fusion of the vesicle with the plasma membrane, a "kiss and run" mechanism was observed at synapses. Here, the vesicle undergoes incomplete fusion and releases its content by the formation of a transient pore. This mechanism in LDCVs would only allow the secretion of small amine transmitters that may be present in these vesicles. Neuropeptides remain trapped inside the vesicle because of their larger size relative to the formed pore and thus are released slowly from LDCVs (Balkowiec \& Katz, 2000; Barg et al, 2002). For these vesicles, it could be shown that a complete fusion of LDCVs is usually required to release the neuropeptide content into the synaptic cleft (Barg et al, 2002). Therefore, it is believed that "kiss and run" may be an important mechanism only for SSVs. 


\section{II.4 Mode of action of neuropeptides}

It is believed that neuropeptides have diverse direct or modulatory effects on its target cell, and when co-released with neurotransmitters, the effects increase dramatically (Kupfermann, 1991). The different types of possible modulations are depicted in Figure II.2. It is possible that both transmitters from one single neuron act on different postsynaptic targets (Figure II.2A) (Yang et al, 1996). However, both types of transmitters normally act on the same postsynaptic membrane (Figure II.2B-D). Usually, neuropeptides bind to G protein-coupled receptors (GPCRs), whereas neurotransmitters activate either GPCRs or ligand-gated ion channels both of which are located in the postsynaptic membrane. Neuropeptides can modulate neurotransmitter triggered postsynaptic responses by altering the gating properties of the ligand-gated ion channels or their response to further signals (Figure II.2B). These changes occur either by direct interaction with the channel or by the activation of second messengers. Furthermore, neuropeptides may alter the number of receptors at the post-synapse or the affinity of the receptor to other (simultaneously) released neurotransmitter (Figure II.2C). In addition to modulating the properties of receptors in the post-synapse, neuropeptides can also regulate the release properties of neurotransmitters and neuropeptides from the presynapse (Figure II.2D). The localization of specific neuropeptide receptors on the presynapse allows for such a feedback regulation of neuropeptide release (Malcangio \& Bowery, 1999) and or the release of the co-transmitter (Glowinski et al, 1993). 

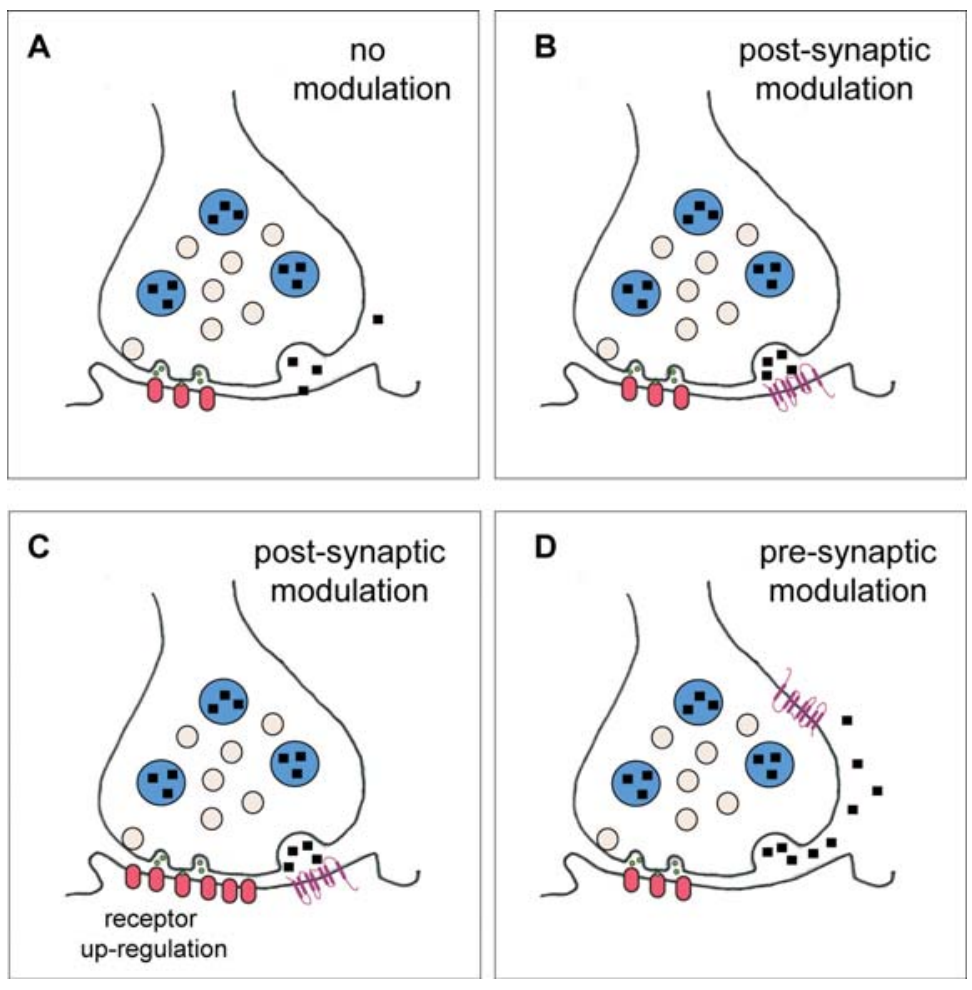

Figure II.2. Simplified representation of possible modulations by neuropeptides at synapses. (A) The post-synaptic membrane lacks neuropeptide specific GPCRs, and thus no modulation at the post-synapse occurs. It is likely that the released neuropeptide acts on a neighboring synapse. (B) For both neurotransmitters and neuropeptides, receptors are located at the postsynaptic membrane and direct interaction or via second messenger can occur. In this way, the gating properties of the neurotransmitter specific channel or its response to further signals can be altered. (C) Neuropeptides may alter the number of receptors at the post-synapse or the affinity of the receptor to other (simultaneously) released neurotransmitter. (D) Activation of pre-synaptic neuropeptide autoreceptors alters release of one or both co-transmitters. Large dense core vesicles depicted in blue, neuropeptides in black squares; synaptic vesicles containing neurotransmitter shown in green. Receptors for neurotransmitter are shown in red. GPCR, G protein-coupled receptor (depicted in purple). Source: picture based on (Salio et al, 2006), Figure 3, (C) SpringerVerlag 2006, with kind permission from Springer Science and Business Media.

\section{II.5 Dense-core vesicles (DCVs) biogenesis}

Contrary to SVs that can be recycled locally at the site of release after exocytosis, DCVs have to be synthesized de novo in the cell body after release (Kim et al, 2006). Despite their importance for the modulation of neurotransmission, neuronal DCV biogenesis is not well understood. Much of our knowledge about neuronal DCVs is based on studies of secretory granules (SG) released from endocrine cells [also known as dense- 
core secretory granules (DCSG), or dense-core granules (DCG)], which are thought to be similar to neuronal DCVs. Figure II.3 summarizes all steps necessary to obtain functional DCVs.

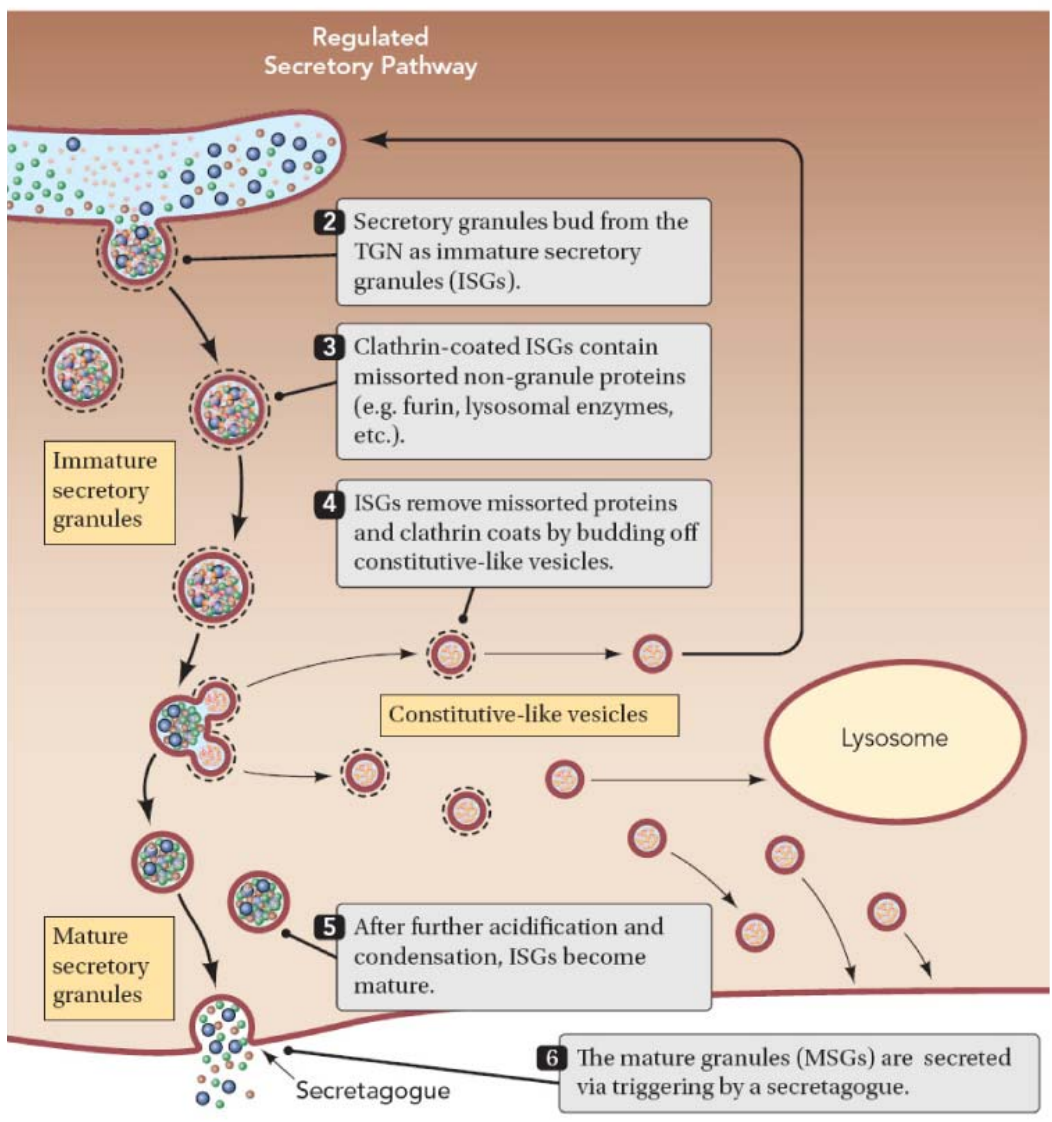

Figure II.3. Steps involved in DCV biogenesis and maturation. General steps during DCV biogenesis in (neuro)endocrine cells are depicted. Source: picture taken from (Kim et al, 2006). Copyright (C) 2006, The American Physiological Society.

Neuropeptides are generated as large precursors at the rough endoplasmic reticulum (rER), inserted into the ER cisternae, where they are properly folded by ERspecific chaperones (Anelli \& Sitia, 2008). Subsequently, they are transported through the Golgi apparatus until they reach the trans-Golgi network (TGN). Here, the precursors are packaged into immature DCVs (iDCVs). These iDCVs are also loaded with processing enzymes and other proteins such as granins (Huttner et al, 1991) (section II.5.2), processing enzymes (e.g. carboxypeptidase E (CPE), proprotein convertases (PC1/3, PC2) (Fricker, 1988; Halban \& Irminger, 1994; Milgram et al, 1997; Seidah \& 
Chretien, 1999), $\mathrm{H}^{+}$V-ATPase (Schoonderwoert \& Martens, 2001), phogrin, and IA-2 (Lu et al, 1996; Wasmeier \& Hutton, 1996).

After the budding of iDCVs from the TGN, a number of maturation steps follow to obtain mature, exocytosis-competent DCVs (mDCVs). During this maturation process the size of iDCVs increases (Tooze et al, 1991), by means of syntaxin 6 and synaptotagmin IV dependent homotypic fusion events of iDCVs to form a functionally distinct compartment from the Golgi (Morvan \& Tooze, 2008).

Furthermore, continuous acidification of DCVs occurs to activate the enzymes (PCs, CPE), which are required for processing of proneuropeptides. The $\mathrm{pH}$ decrease from the TGN ( $\mathrm{pH} \sim 6.5-6.2$ ) via iDCVs ( $\mathrm{pH} \sim 6.3-5.7$ ) to $\mathrm{mDCVs}$ ( $\mathrm{pH} \sim 5.5-5.0$ ) is achieved by increases in the density of $\mathrm{H}^{+}$V-ATPase pumps and by decreases in $\mathrm{H}^{+}$ permeability through the membranes (Wu et al, 2001a). It has been shown that an acidic $\mathrm{pH}$ is not only crucial to activate processing enzymes, but also required for the formation of DCVs (Tanaka et al, 1997; Taupenot et al, 2005).

It has been shown that syntaxin 6 and synaptotagmin IV, along with mis-sorted lysosomal enzymes, furin and other proteins such as constitutive secretory pathway proteins are removed by budding of clathrin coated vesicles from maturing DCVs (Kim et al, 2006; Tooze et al, 1991). Clathrin binding from DCVs is dependent on the adaptor complex AP-1 whose recruitment is mediated by the small GTPase Arfl (Dittie et al, 1996). Previously, it was observed that other clathrin adaptor proteins such as GGAs (Golgi associated, $\gamma$ ear containing, ADP ribosylation factor binding protein) are also involved in this remodeling step (Kakhlon et al, 2006). The fate of these removed, constitutive vesicles is diverse (Kim et al, 2006): In some cases, these vesicles undergo constitutive secretion, whereas in other cases they are destined to fuse with endosomes and some are transported back to the TGN, which carry TGN-resident processing enzymes, like furin (Dittie et al, 1997; Molloy et al, 1994).

This clathrin-dependent membrane remodeling process functions as a proofreading mechanism to ensure proper DCV content and correct membrane composition of mDCVs (Morvan \& Tooze, 2008). This is necessary, since it has been shown that only mDCVs are able to undergo efficient, stimulus-dependent exocytosis (Eaton et al, 2000). 
The final steps of DCV maturation, prior to fusion, are condensation of cargo molecules by further acidification and removal of water (Kim et al, 2006). After all maturation steps, mDCVs are transported along microtubules from the cell body to their releasing sites, where they are stored until they undergo stimulus dependent exocytosis (Park et al, 2009).

\section{II.5.1 Lipids necessary for vesicle budding at the TGN}

It has been shown that lipids like diacylglycerol (DAG) and phosphatidic acids (PAs) are important to initiate vesicle budding. Due to the conical molecular shape of these molecules at conditions found in the TGN provides sufficient force to induce a negative curvature of the Golgi membrane (Corda et al, 2002; Shemesh et al, 2003). Moreover, phosphoinositides are also contributors to vesicular budding by recruiting adaptor proteins whose interaction facilitates membrane traffic (Godi et al, 2004). A significant role in vesicle formation plays cholesterol. Together with other lipids such as sphingomyelin, it forms "lipid rafts" microdomains, from which vesicles bud at the TGN (Dhanvantari \& Loh, 2000).

\section{II.5.2 Granins and its role in aggregation within DCVs}

Granins represent an important protein family that is crucial during vesicle formation. These proteins are abundant in DCVs. Well-studied representatives are chromogranin A (CgA), B (CgB), and secretogranin II-IV (SgII-IV) (Taupenot et al, 2002). Granins are rich in acidic amino acids, possess a high binding capacity for $\mathrm{Ca}^{2+}$, and self-aggregate at mildly acidic $\mathrm{pH}(\mathrm{pH} 5-6)$ and high $\mathrm{Ca}^{2+}$ concentrations (10-40 $\mathrm{mM}$ ) (Kim et al, 2006). These conditions are found in the TGN (Colomer et al, 1996; Tooze et al, 1991). This aggregation behavior of granins is essential in the sorting of DCV cargo, since it was shown that cargo proteins can be efficiently incorporated into the granin aggregates, whereas constitutively secreted proteins are excluded (Colomer et al, 1996; Tooze et al, 1991). 
Previously, it has been shown that secretogranin III (SgIII) plays an essential role in DCV biogenesis. It possesses three functional domains: a cholesterol-binding domain, a CgA-binding domain, and a carboxypeptidase E (CPE)-binding domain (Figure II.4), allowing simultaneously binding to $\mathrm{CgA}$, cholesterin-rich membrane and CPE (Hosaka et al, 2005). In addition to its processing activity, CPE is predicted to be a sorting receptor for DCVs (Loh et al, 2002). By utilizing all these three domains, SgIII may function as a molecular bridge between the aggregated core and the cholesterol-rich membrane of DCVs (Hosaka \& Watanabe, 2010) (section II.6.).

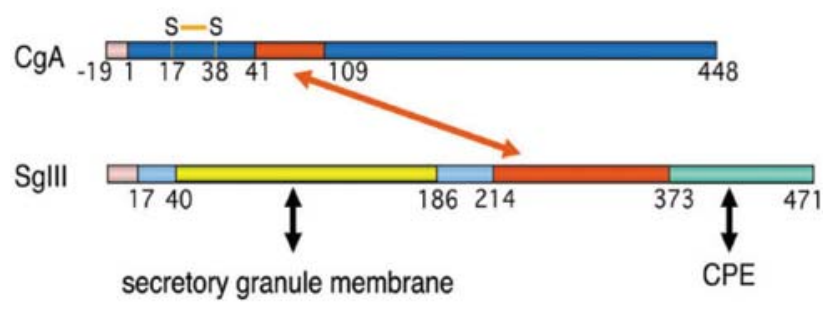

Figure II.4. Protein domain structure of CgA and SgIII. Rat SgIII (471 aa) contains at least three functional domains, including a cholesterol-binding domain (aa 40-186, yellow), a CgAbinding domain (aa 214-373, red), and a CPE-binding domain (aa 374-471 turquois). The CgAbinding domain of SgIII is specifically associated with an SgIII-binding domain within the rat CgA molecule (CgA 41-109). CgA, chromogranin A; SgIII, secretogranin III; CPE carboxypeptidase E. Source: picture taken from (Hosaka \& Watanabe, 2010). Copyright (C) Endocrine Journal 2010.

\section{II.6. Models for DCV formation}

Two main models have been proposed to explain how the appropriate cargos are sorted into newly generated DCVs: sorting by entry and sorting by retention. The sorting by entry model assumes the existence of sorting signals and receptors that would actively sort cargo into forming DCVs (Arvan \& Castle, 1998; Borgonovo et al, 2006; Kim et al, 2006; Tooze et al, 2001). Such short signal motifs have been identified in DCV cargos such as provasopressin, pro-oxytocin, pro-opiomelanocortin, $\mathrm{CgA}$, and $\mathrm{CgB}$, which are sufficient for DCV targeting (Cool et al, 1995; Glombik \& Gerdes, 2000; Huttner et al, 1991; Tooze, 1998). In contrast, the sorting by retention model suggests that DCV cargo could passively enter maturing DCVs and then be retained during DCV maturation either 
by active retention in lipid domains or by its aggregation within iDCVs (Glombik \& Gerdes, 2000; Hosaka \& Watanabe, 2010). It has been shown in the case of SgIII that there are direct interactions between aggregated DCV cargos and cholesterol-rich membrane domains of DCVs (Hosaka \& Watanabe, 2010). In this way, SgIII binds to forming micro-aggregates containing $\mathrm{CgA}$ and proneuropeptides through protein-protein interactions and keeps these aggregates at the cholesterol-rich membrane, which contains processing enzymes (Figure II.5, step 1). In this way, proneuropeptides can be easily transferred from the premature aggregate to the neighboring processing enzymes. After processing, mature neuropeptides aggregate and are incorporated into the neighboring aggregates, which are tethered to the lipid raft (Figure II.5, step 2). Thus, the aggregates increase in size and density. After formation of a vesicle is completed, aggregates are further concentrated inside the DCV forming the dense-core (Figure II.5, step 3).

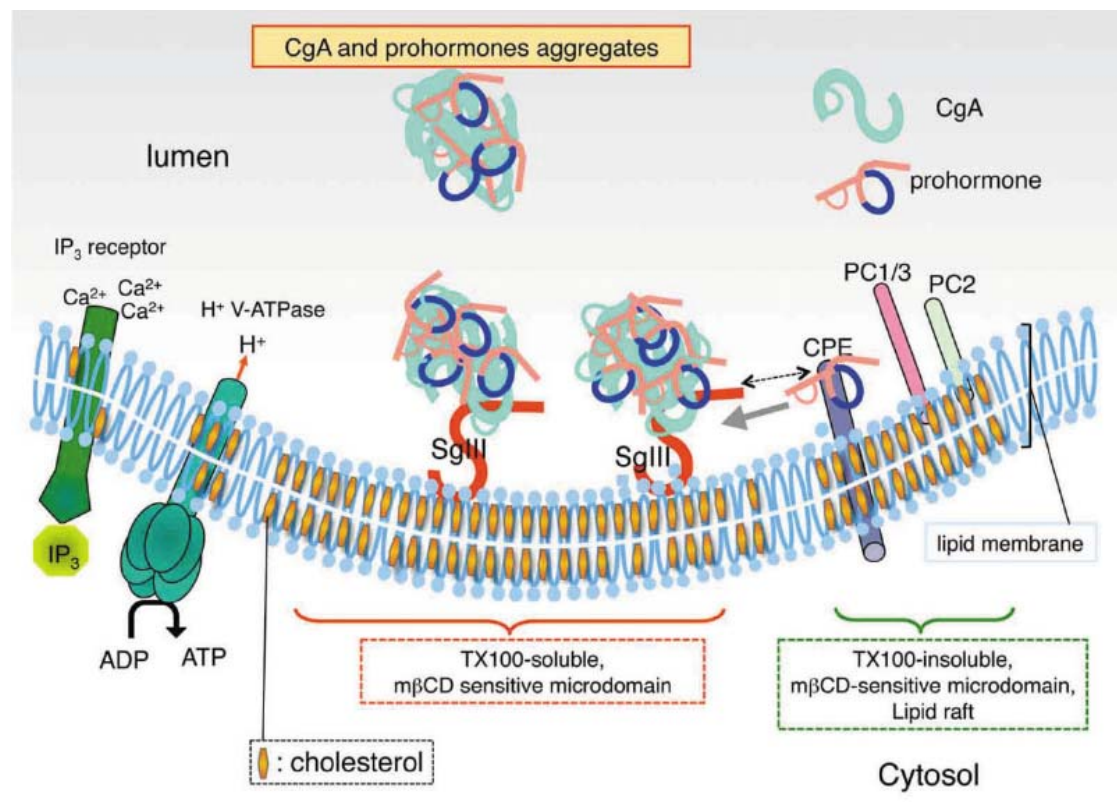

Figure II.5. Key steps for neuropeptide sorting mediated by SgIII and cholesterol-rich membrane at the TGN.

STEP 1. Micro-aggregates, including neuropeptides/prohormone and $\mathrm{CgA}$, can be tethered to a cholesterol-rich membrane domain at the TGN or the immature SG with SgIII, which can bind to both $\mathrm{CgA}$ and cholesterol. In parallel, processing enzymes (CPE, PC1-3) are also recruited to the raft-like microdomains. These function as a membrane platform, on which the processing enzymes and a large amount of prohormones in the micro-aggregate are closely gathered. Source: picture taken from (Hosaka \& Watanabe, 2010). Copyright (C) Endocrine Journal 2010. 


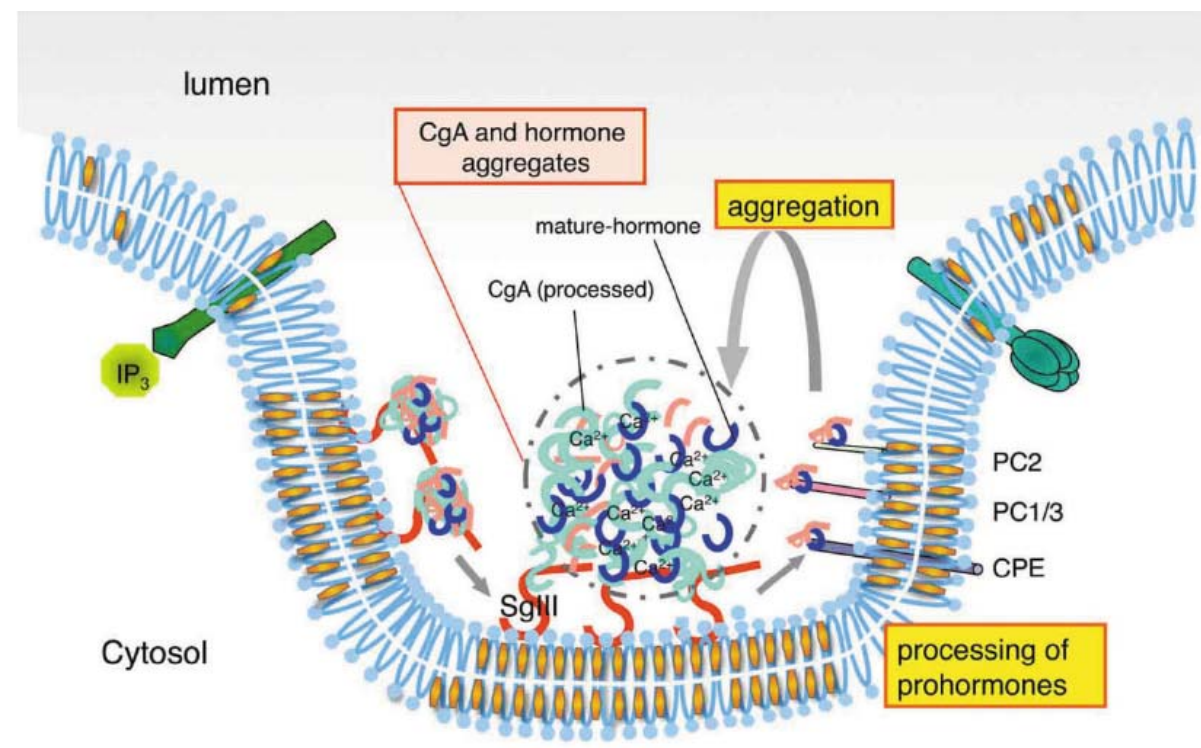

STEP 2. On the cholesterol-rich membrane platform, prohormones in the premature aggregates are transferred to the neighboring processing enzymes and then processed to small fragments. In turn, processed mature hormones are incorporated into the neighboring premature aggregates, including $\mathrm{CgA}$, tethered to the membrane domain with SgIII. As the processed mature hormones and granin fragments are incorporated, the aggregate matures to be larger in size and concentrated with hormones. Source: picture taken from (Hosaka \& Watanabe, 2010). Copyright (C Endocrine Journal 2010.

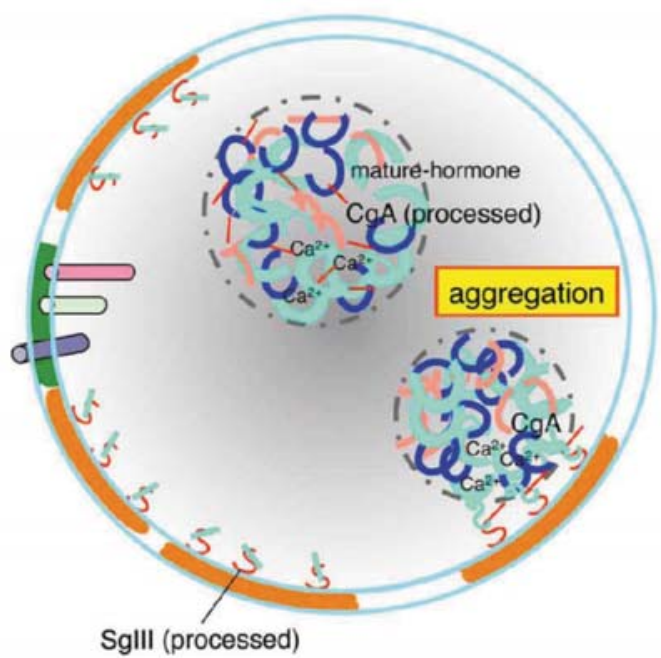

STEP 3. After the secretory granule is established as an independent compartment, mature hormones and granin fragments move from the periphery to the inside of the secretory granules where they are further concentrated to form a dense core aggregate. Within the mature aggregate, neuropeptides/hormones are ready to be released upon a membrane fusion event. Source: picture taken from (Hosaka \& Watanabe, 2010). Copyright (C) Endocrine Journal 2010. 


\section{II.7 Regulation of DCV biogenesis}

After stimulus-dependent exocytosis of DCVs, the DCV pool has to be replenished by the generation of newly synthesized DCVs in the (neuronal) cell bodies (Tooze, 1991). Regulation of DCV biogenesis occurs at different various levels ranging from the initial transcription of DCV related genes to the final maturation of DCVs (Kim et al, 2006). Each regulatory step influences the quantity of available DCV proteins in the cell and therefore, determines the amount of formed DCVs.

It could be observed that after secretion, the expression of cargo molecules (neuropeptides) and proteins involved in DCV biogenesis are upregulated (Kim et al, 2006). It has been shown that signals that trigger exocytosis, called secretogogs, activate the transcription of mRNAs encoding DCV proteins in the nucleus, possibly through a feedback mechanism (Eiden et al, 1984; Mahapatra et al, 2003; Mahata et al, 2003; Tang et al, 1997).

Besides this transcriptional regulation of DCV proteins, a post-transcriptional control mechanism was observed. For many 3'-UTRs of DCV mRNAs, a binding site for polypyrimidine-tract binding protein (PTB) was identified (Knoch et al, 2004). It was reported that such a binding stabilizes the mRNA, protects it against early degradation and results in increased translation of DCV proteins and DCV formation in insulinsecreting $\beta$-cells (Knoch et al, 2004).

Interestingly, $\mathrm{CgA}$ itself might be actively involved in the regulation of DCV formation by an unknown mechanism (Kim et al, 2006; Kim et al, 2005; Mahapatra et al, 2005). It has been shown that $\mathrm{CgA}$ protects against active degradation of DCV proteins in the Golgi by induction of a protease inhibitor, protease nexin-1 (PN-1) (Kim \& Loh, 2006).

Recent studies indicate that another protein, insulinoma-associated protein 2 (IA-2 or ICA512), is involved in the regulation of formation of DCVs in insulin-secreting $\beta$ cells (Harashima et al, 2005). This transmembrane protein is present on DCV membranes and upon secretion of DCV, IA-2 is inserted into the plasma membrane where its cytosolic tail is cleaved off in a $\mathrm{Ca}^{2+}$-dependent manner. This fragment is then 
translocated into the nucleus, inducing insulin expression in $\beta$-cells (Harashima et al, 2005).

All the described findings indicate that DCV biogenesis in (neuro)endocrine cells is regulated at transcriptional, post-transcriptional, and post-translation levels, which are summarized in Figure II.6.

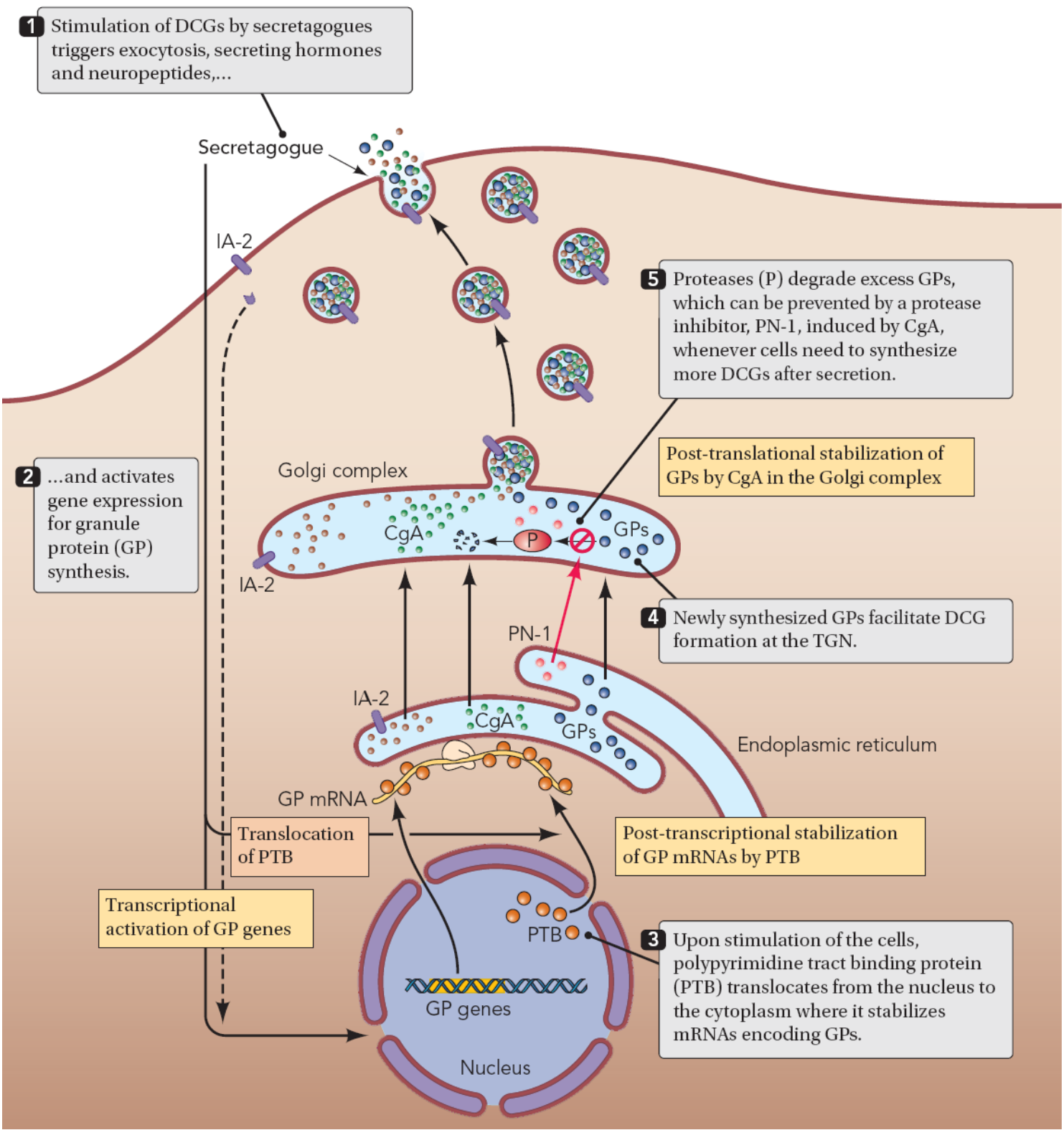

Figure II.6. Regulation of DCV biogenesis. To maintain the steady-state pool of DCVs as well as to replenish amount of stored DCVs after secretion, DCV biogenesis is regulated at different levels: transciptional, post-transcriptional, and post-translational. Source: picture taken from (Kim et al, 2006). Copyright (C) 2006, The American Physiological Society. 


\section{II.8 Role of RAB-2 and its effector RIC-19 in DCV maturation in C. elegans}

Previously, our lab and others have demonstrated that the small GTPase RAB-2 is crucial for DCV maturation in C. elegans (Edwards et al, 2009; Sumakovic et al, 2009). These studies suggested a role of RAB-2 for the retention of soluble and transmembrane cargos within maturing DCVs. Loss of RAB-2 function resulted in mis-sorting of cargo into the endosomal-lysosomal pathway (Edwards et al, 2009; Sumakovic et al, 2009) (Figure II.7A). Blockage of this pathway by over-expressing a constitutively active, GTPbound RAB-5 (Q79L) could rescue the loss of cargo (Sumakovic et al, 2009) (Figure II.7B). The same effect was observed when endosomal PI(3)P was sequestered by expressing the FYVE domain of EEA-1 (early endosome antigen-1), which leads to blockage of early-endosomal function (Edwards et al, 2009). These data indicate that inhibition of the constitutive-like secretory pathway from maturing DCVs during biogenesis suppresses DCV maturation defects in unc-108/rab-2 mutants.

A

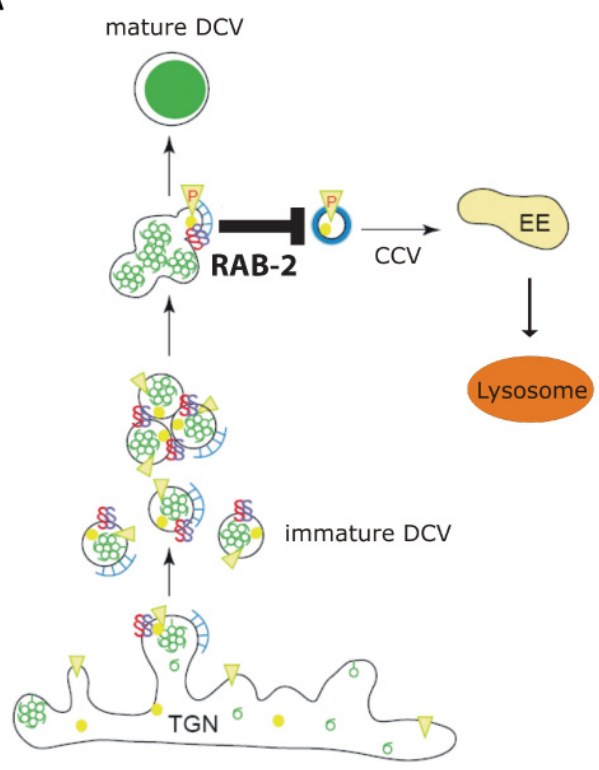

B

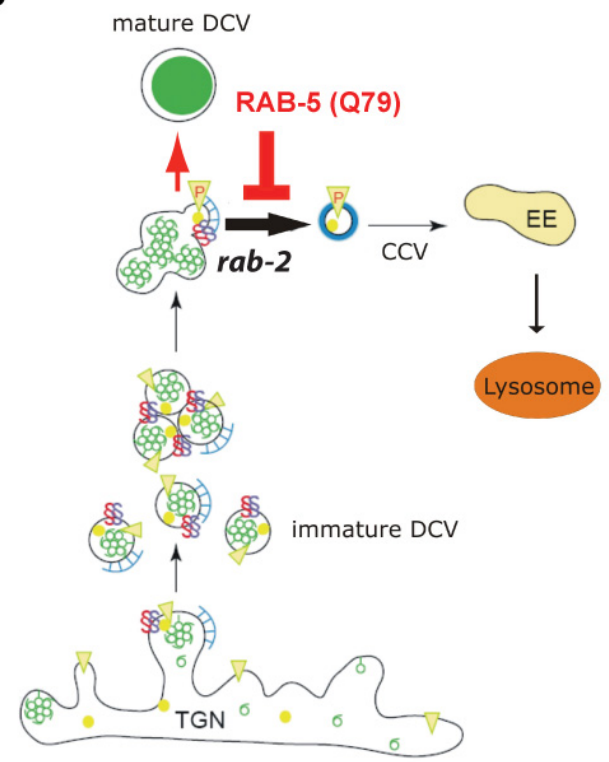

Figure II.7. The small GTPase RAB-2 is involved in the retention of cargo during DCV maturation. (A) RAB-2 prevents the loss of cargo during DCV maturation into the endosomallysosomal pathway. (B) Loss of RAB-2 (indicated by rab-2) leads to loss of cargo into endosomal-lysosomal pathway. Over-expression of constitutively active, GTP-bound RAB-5 (Q79L) inhibits early endosomal function and suppresses loss of cargo in rab-2 mutants (red arrow). Source: picture adapted from (Tooze et al, 2001), with permission from Elsevier. 
Co-localization studies revealed that RAB-2 is localized to the Golgi complex and cannot be detected at synapses, indicating that RAB-2 function is mainly restricted to the neuronal cell body (Sumakovic et al, 2009).

Interestingly, RAB-2 was assigned to have a role in acidification of phagosomes during the degradation of engulfed apoptotic cells in the germ line (Mangahas et al, 2008). However, it is unlikely that RAB-2 is also involved in the acidification of DCVs, since processing enzymes are only active under acidic $\mathrm{pH}$ conditions and neuropeptides are fully processed in unc-108/rab-2 mutants, evaluated by a Matrix-assisted laser desorption/ionization time of flight (MALDI TOF) mass spectrometry analysis (Sumakovic et al, 2009). Therefore, RAB-2 likely does not have a role in neuropeptide processing.

Furthermore, EM analysis discovered no changes in DCV numbers at synapses, indicating that RAB-2 might not be involved in the formation of DCV at the Golgi (Sumakovic et al, 2009).

It was shown that the C. elegans ICA69 homolog, RIC-19, is an effector of RAB2 (Sumakovic et al, 2009). The localization of RIC-19 is dependent on RAB-2. Only GTP-bound RAB-2 recruits RIC-19 to Golgi membranes, whereas GDP-bound RAB-2 causes cytosolic distribution of RIC-19 in neurons (Sumakovic et al, 2009). Interestingly, ric-19 mutants displayed similar DCV maturation defects to unc-108/rab-2 mutants. By utilizing their BAR (Bim/amphiphysin/Rvs) domains, RIC-19 and its human homolog ICA69 are predicted to bind to curved membranes found on vesicular structures (Gallop \& McMahon, 2005; Habermann, 2004; Zimmerberg \& McLaughlin, 2004). Therefore, RAB-2 together with RIC-19 are predicted to create and stabilize membrane domains on maturing DCVs that might be important to maintain specific cargo within these vesicular structures (Sumakovic et al, 2009). 


\section{II.9 The family of small Rab GTPases}

Rab2 belongs to the family of small Rab GTPases. With over 60 members in humans, 28 members in C. elegans and 11 members in yeast, small Rab GTPases constitute the largest family of the Ras superfamily (Hutagalung \& Novick, 2011; Zerial \& McBride, 2001). This Ras superfamily consists of five families: Arf, Rab, Ran, Ras, and Rho GTPases (Wennerberg et al, 2005). Rab (Ras-like proteins in rat brain) GTPases are master regulators of intracellular membrane trafficking, mediating all steps of vesicle transport (section II.11). It has been shown that Rab proteins localize to distinct compartments in the cell and regulate vesicle transport between organelles of the endocytic and secretory pathways (Zerial \& McBride, 2001) (Figure II.8).

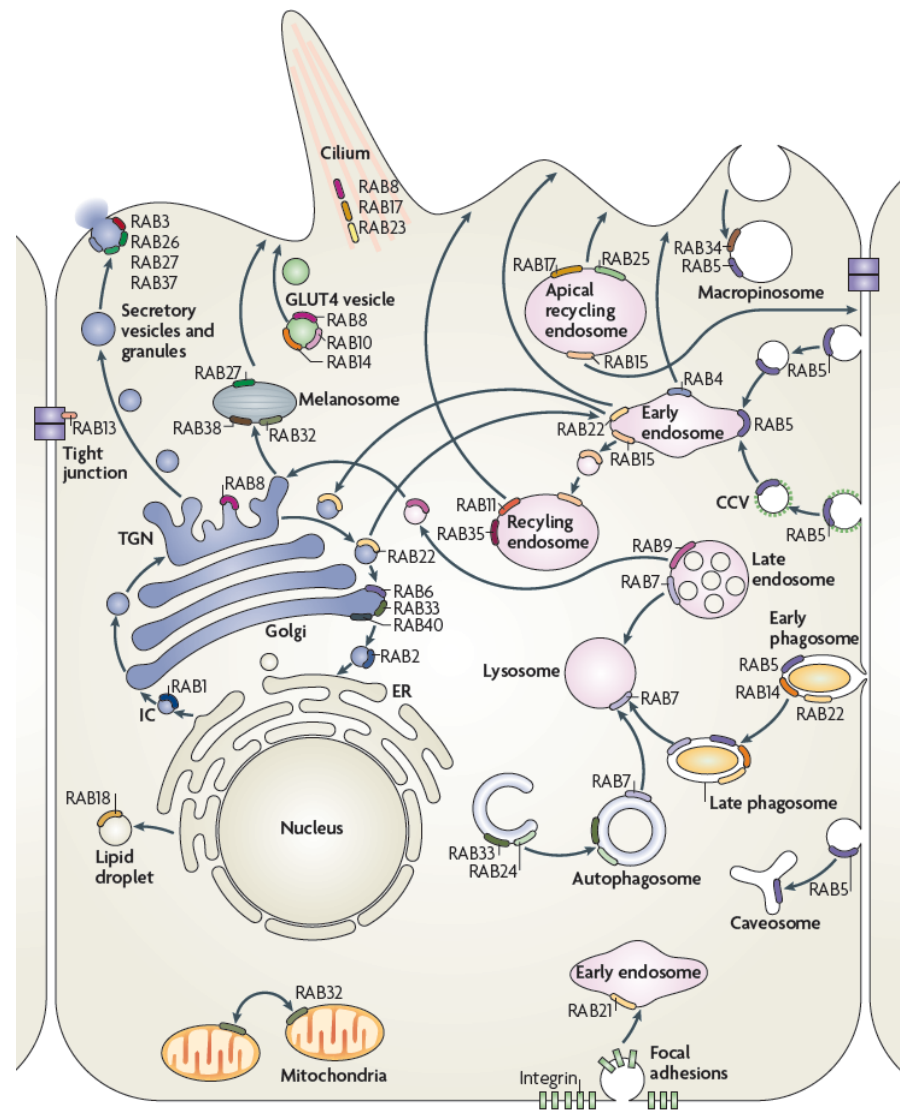

Figure II.8. The intracellular localization of Rab GTPases. They regulate vesicular transport between organelles. Their localizations label distinct intracellular compartments in eukaryotic cells. For detailed information on each Rab function, please see (Hutagalung \& Novick, 2011). $\mathrm{CCV}$, clathrin-coated vesicle; ER, endoplasmic reticulum; IC, ER-Golgi intermediate compartment; TGN, trans-Golgi network. Source: picture reprinted by permission from Macmillan Publishers Ltd: (Stenmark, 2009). 
Ras (모t sarcoma) proteins act within signaling networks in the regulation of gene expression, cell proliferation, differentiation, and survival (Wennerberg et al, 2005). The Rho (ㅁas homologous) family also plays an essential role in extracellular-stimulusmediated signaling networks in order to control actin organization, cell cycle progression and gene expression (Etienne-Manneville \& Hall, 2002). Ran (ass-like nuclear) proteins are required for the nucleocytoplasmic transport of both proteins and RNA (Weis, 2003). Furthermore, Ran GTPases are involved in mitotic spindle assembly, DNA replication and nuclear envelope assembly ( $\mathrm{Li}$ et al, 2003). Like Rab GTPases, Arf (ADPribosylation factor) proteins are also involved in the regulation of vesicular transport by recruiting coat proteins to membranes (Wennerberg et al, 2005).

\section{II.9.1 The conserved protein structure of Rab GTPases}

Protein crystallization studies of different Rab GTPases revealed similarities and differences in the protein structure of Rab GTPases (Figure II.9). Like the other members of the Ras superfamily, Rab proteins possess a common GTPase fold, consisting of sixstranded $\beta$-sheet surrounded by five $\alpha$-helices (Itzen $\&$ Goody, 2011). The N-terminus of $\mathrm{Rab}$ proteins contains the so-called P-loop, which is required for binding phosphate and coordinating $\mathrm{Mg}^{2+}$ (Dumas et al, 1999).

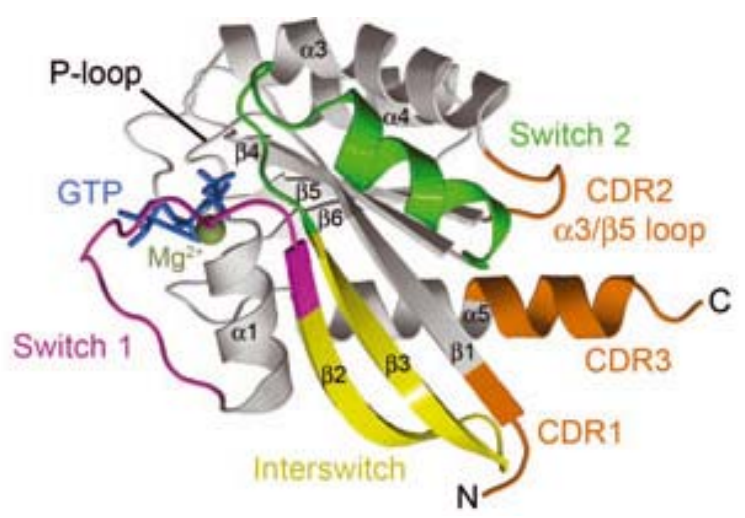

Figure II.9. General protein structure of Rab GTPases. Overall structure of the Rab3 GTPase with its functional regions that are indicated by different colors. Source: picture taken with permission from (Lee et al, 2009). DOI: 10.1111/j.1600-0854.2009.00942.x. C 2009 John Wiley \& Sons $\mathrm{A} / \mathrm{S}$. 
Further important regions of the GTPase fold are the switch I and II regions that are in contact with the $\gamma$-phosphate of the GTP (Hutagalung \& Novick, 2011). Dependent on the nucleotide-binding state, these switches undergo dramatic changes in their conformation (Milburn et al, 1990; Schlichting et al, 1990). While the switch regions show a disordered structure when bound to GDP (Figure II.10A), upon GTP binding these regions change to an ordered structure (Figure II.10B).

A

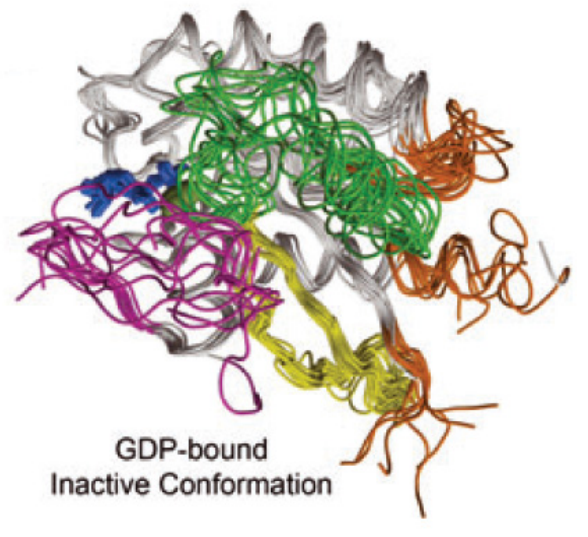

B

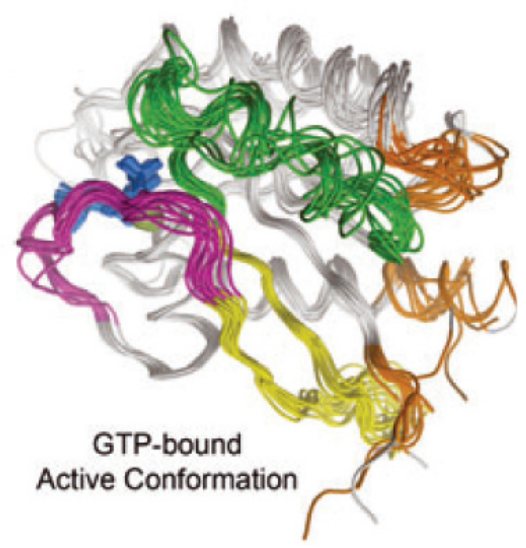

Figure II.10. General protein structure of Rab GTPases. (A) Comparison of inactive GDPbound Rab GTPase structures after superposition with Rab2. Note that both switch regions (purple and green) are poorly ordered. (B) Comparison of active Rab GTPase structures (bound to non-hydrolysable analog of GTP, GppNHp) after superposition with Rab3. Both switch regions adopt stable active conformations. Note that switch II exhibits large conformational differences between Rab GTPases. For the color codes of domains, see Figure II.9. Source: picture taken with permission from (Lee et al, 2009). DOI: 10.1111/j.1600-0854.2009.00942.x. C 2009 John Wiley \& Sons A/S.

Besides both switch domains, the $\alpha 3 / \beta 5$ loop (Figure II.9,10, brown) that is located adjacent to the switch II region shows the highest structural heterogeneity of the Rab structure (Hutagalung \& Novick, 2011). The variation in this loop is likely to account for the recruitment of the diverse set of effectors to each Rab protein. Close to the GTPase fold, a hyper-variable region followed by the CAAX box is found in the 
protein structure of Rab GTPases (Hutagalung \& Novick, 2011). This CAAX box normally includes two cysteine residues, which geranylgeranyl moieties are covalently linked to. The hyper-variable region is essential for the regulated localization of Rab GTPases to specific membranes, whereas the prenyl moieties allow the reversible insertion of these proteins into membranes (Hutagalung \& Novick, 2011) (Figure II.11).

A

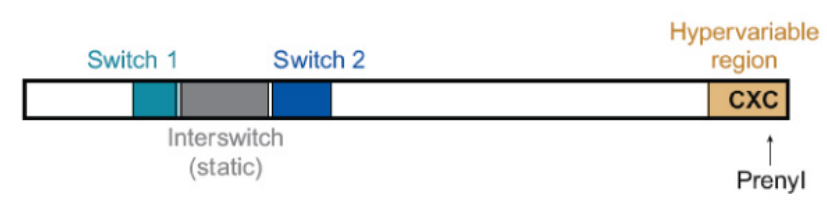

B

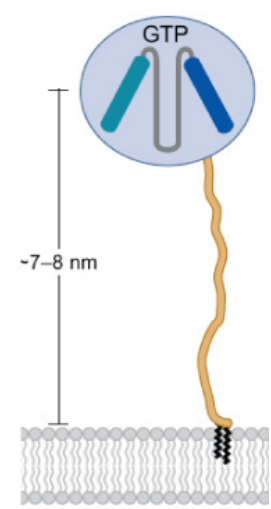

Figure II.11. Prenylation of the C-terminus of Rab proteins leads to reversible membrane localization. (A) Schematic protein domain representations of a typical Rab GTPase. Rab proteins are modified with prenyl groups at their $\mathrm{C}$-terminal cysteines allowing for insertion into membranes (B). Their extended hyper-variable region (brown) connecting the protein to the lipid anchor allows bound effectors to move further from the bilayer. Source: picture adapted from (Gillingham \& Munro, 2007) with permission from ANNUAL REVIEW OF CELL AND DEVELOPMENTAL BIOLOGY.

Additional conserved stretches of amino acids, RabF1-F5, were identified in Rab proteins, which are specific to this Ras-like family and are not found in other GTPases (Pereira-Leal \& Seabra, 2000). Furthermore, subfamily specific regions (RabSF1-4) have also been discovered, which define ten groups of Rab subfamilies (Pereira-Leal \& Seabra, 2000). It is believed that RabF stretches are important for effectors and regulators to discriminate between the nucleotide-binding state of the Rab, while RabSF stretches are required for specificity of effector and regulator binding (Pereira-Leal \& Seabra, 2000). 


\section{II.9.2 Regulation of Rab GTPase activity}

The ability of Rab GTPases to exist either in an inactive guanosine nucleotide diphosphate (GDP)-bound state or an active guanosine nucleotide triphosphate (GTP)bound state, allows them to act as molecular switches (Figure II.12). Dependent on the bound guanosine nucleotide, Rab proteins undergo dramatic changes in their threedimensional protein structure. In their active, GTP-bound state Rab proteins can intact with effector proteins, whose interaction is temporally and spatially regulated by the (intrinsic and catalyzed) rates of nucleotide exchange and hydrolysis of the Rab protein. Diverse effector proteins for Rab GTPases have been identified, ranging from tethering factors, kinases, phosphatases to motor proteins [reviewed in (Hutagalung \& Novick, 2011)].

In general, Rab proteins have a low intrinsic GTP binding and GTP hydrolysis activity (Pfeffer, 2005). Therefore, accessory proteins are needed to either activate or inactivate Rab GTPases (Figure II.12). The exchange of GDP with GTP is catalyzed by the action of guanine nucleotide exchange factors (GEFs). GEFs bind to the switch regions and facilitate GDP release. Due to the high cytosolic concentration of GTP in the cell, it immediately binds to the Rab, after GDP has been released from the nucleotidebinding pocket. The intrinsic GTP-hydrolysis of Rab proteins is accelerated by GTPaseactivating proteins (GAPs) leading to the release of inorganic phosphate $\mathrm{P}_{\mathrm{i}}$ (Pfeffer, 2005) (Figure II.12).

GDP-bound Rab proteins are recognized by Rab GDP dissociation inhibitors (GDI) that chaperones the geranylgeranyl moieties within the cytosol and mediates the transport of Rab GDP to their destined donor compartment (Soldati et al, 1994; Ullrich et al, 1994; Ullrich et al, 1993) (Figure II.12). 


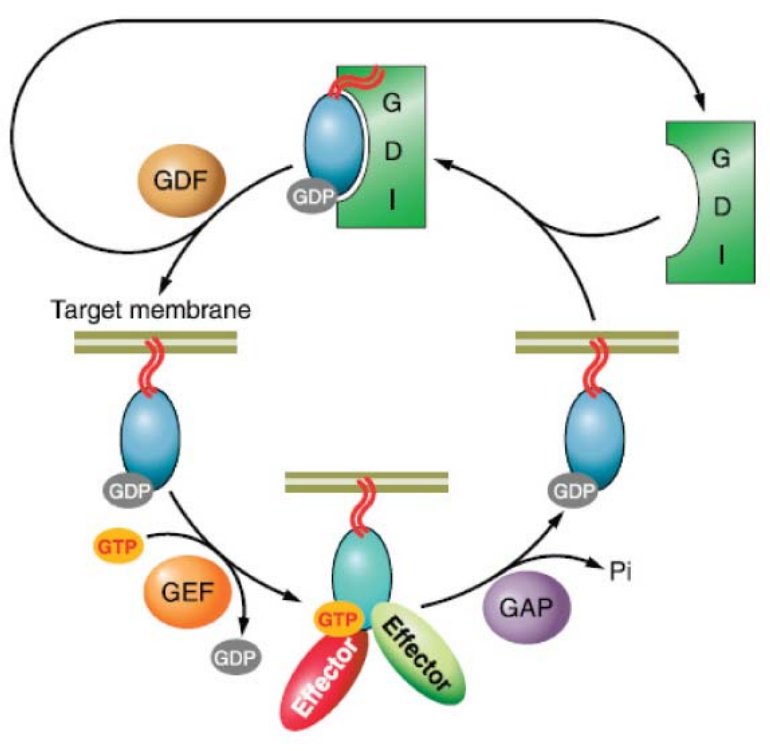

Figure II.12. The Rab cycle. Rab GTPases cycle between an active, GTP-bound state and an inactive, GDP-bound state. Activation of the Rab leads to interaction with effector proteins that mediate membrane traffic in the pathway regulated by its associated Rab. For the exchange of GDP with GTP guanine nucleotide exchange factors (GEFs) are required in order to activate Rab proteins. GTP hydrolysis is accelerated by GTPase activating proteins (GAPs) leading to inactivation of the Rab. The inactivated $\mathrm{Rab}$ is removed from the membrane by guanine nucleotide dissociation inhibitor (GDI) and is transported to the target membrane. By the action of GDI dissociation factor (GDF) the Rab is released from GDI and inserted into the target membrane to start a new cycle. The C-terminus located prenyl tail is indicated by red wave lines. Source: picture adapted from (Hutagalung \& Novick, 2011). Copyright (C) 2011 the American Physiological Society.

\section{II.9.3 Function of Rab GTPases in vesicular transport}

It is well established that an active Rab cycle is important for Rab function. In the active state, Rab proteins interact with various effector proteins. These effectors function during all aspects of vesicular membrane traffic: a) vesicle formation, b) vesicle uncoating, c) vesicle movement, d) vesicle tethering, and e) vesicle fusion (Stenmark, 2009) depicted in Figure II.13. 


\section{a) Cargo selection and vesicle formation}

Several Rab proteins have been implicated in the process of recruiting coat complexes to membranes and facilitating vesicle formation (Hutagalung \& Novick, 2011). Rab9, for example, regulates retrograde transport of mannose-6-phosphate receptors from late endosomes to the TGN (Lombardi et al, 1993). Activated Rab9 interacts with the effector TIP47 that binds to the cytoplasmic tail of mannose-6phosphate receptors. This interaction is essential for the retrograde transport of the receptor to the TGN (Aivazian et al, 2006; Carroll et al, 2001; Diaz \& Pfeffer, 1998). During vesicle formation, the binding between Rab9 and TIP47 enhances the affinity of TIP47 for the receptor (Carroll et al, 2001).

\section{b) Vesicle uncoating}

Uncoating is an important step before vesicle fusion with the target membrane can occur. It has been previously shown that Rab5 is involved in uncoating of clathrin coated vesicles (CCVs) [reviewed in (Hutagalung \& Novick, 2011)]. Furthermore, Ypt1, the yeast homolog of Rab1, is required for ER-to-Golgi traffic by recruiting factors important for uncoating of COPII vesicles (Lian et al, 1994; Moyer et al, 2001).

\section{c) Vesicle motility}

Rab proteins are also involved in the motility of vesicles along cytoskeletal structures like actin filaments or microtubules. Rab6 interacts with its effector Rabkinesin-6, a kinesin-like protein, which is important for microtubule-dependent transport of vesicles and organelles (Echard et al, 1998). It was shown that Rab11 regulates plasma membrane recycling through its interaction with its effector, Rab11FIP2, which binds to myosin Vb (Hales et al, 2002). Furthermore, Rab27a is involved in the transport of melanosomes to the plasma membrane by recruiting its effector 
melanophilin/Slac2-a that interacts with the actin motor, myosin Va (Bahadoran et al, 2001; Hume et al, 2001; Strom et al, 2002; Wu et al, 2001b).

d) Vesicle tethering

Before vesicles fuse, they have to be tethered to the target membrane. Tethering complexes are important in this step, because they ensure fidelity of transport pathways (Hutagalung \& Novick, 2011). Many studies show that different tethering complexes are effectors of Rab GTPases regulating fidelity of vesicle fusion [reviewed in (Hutagalung \& Novick, 2011)].

e) Vesicle fusion

Rab proteins are also involved in the last step of vesicle transport, in vesicle fusion. They regulate SNARE-(soluble N-ethylmaleimide-sensitive factor attachment protein receptors) mediated fusion of the vesicles to the target membrane. Effectors of Rab GTPases are either SNARE proteins (Schardt et al, 2009) or proteins that mediate SNARE function [reviewed in (Hutagalung \& Novick, 2011)]. 


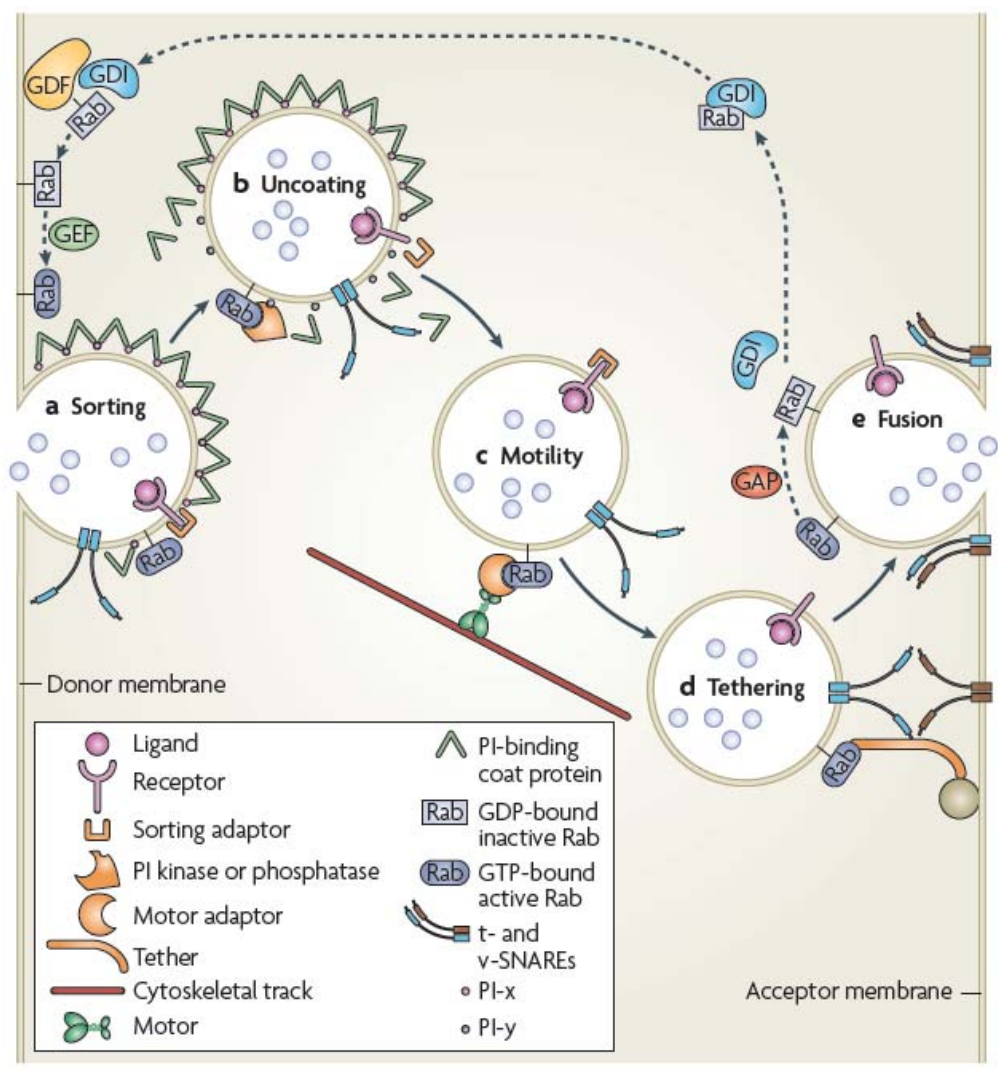

Figure II.13. Rab GTPase functions in vesicle trafficking. Distinct membrane trafficking steps that can be controlled by Rab GTPases (blue) and their effectors (orange). (a) Rab proteins recruit an adaptor protein to sort a receptor into a budding vesicle. (b) Through recruitment of effectors, $\mathrm{Rab}$ proteins cause uncoating through the dissociation of coat proteins. (c) Rab GTPases can mediate vesicle transport along actin filaments or microtubules (cytoskeletal tracts) by recruiting motor proteins or motor adaptors. (d) Rab GTPases can mediate vesicle tethering by recruiting tethering complexes. (e) Rab proteins (in)directly interact with SNAREs to mediate SNAREdependent fusion of vesicles with the plasma membrane. Source: picture reprinted by permission from Macmillan Publishers Ltd: (Stenmark, 2009).

\section{II.9.4 Specific functions of Rab GTPases in neurons}

Neurons are highly-specialized cells that have a demand for highly regulated membrane trafficking. Cargo that is required for synaptic transmission, remodeling and neurite outgrowth has to be generated in the cell body and transported over long distances to the axons. 
It has been shown that Rab GTPases are involved in several aspects of neuron specific trafficking processes, like polarized outgrowth of neurites, neuronal anterograde transport, axonal endocytosis, retrograde transport, and SV exocytosis (Ng \& Tang, 2008). Different Rab proteins could be identified on purified SVs from rat brain, including Rab3a/b/c, and Rab27b (exocytotic Rab machinery of SVs) and Rab4b, Rab5a/b, Rab10, Rab11b, and Rab14 (endocytic Rab machinery of SVs) (Pavlos et al, 2010). The involvement of Rab3 and Rab27 for tethering SVs to the synaptic density and exocytosis has been shown before (Geppert et al, 1994; Gracheva et al, 2008; Li et al, 1994; Mahoney et al, 2006; Ng \& Tang, 2008).

Previously, we and others could show that Rab GTPases are also required for DCV trafficking. As described above, Rab2 and Rab5 play a role in neuronal DCV maturation in C. elegans (Edwards et al, 2009; Sumakovic et al, 2009) (section II.8). Interestingly, the isoform Rab3D is localized to SGs in non-neuronal tissues, like pancreatic cells. Since Rab3D is not expressed in neurons, it was hypothesized that in secretory cells Rab3D might have the same function as Rab3A in neurons (Morvan \& Tooze, 2008). However, it appears that Rab3D is not involved in SG exocytosis, but in SG maturation (Riedel et al, 2002). Rab3D-deficient mice showed an increased size of SGs in pancreatic cells, whereby the intragranular protein concentration seems to be unchanged (Riedel et al, 2002). In C. elegans an involvement in SV trafficking has been shown for RAB-3 (Richmond, 2005), however, whether it has also a role in DCV maturation has to be elucidated, since only one RAB-3 isoform exists in C. elegans (www.wormbase.org).

\section{II.10 Studying DCV function in C. elegans}

The nervous system of C. elegans is less complex than that one of higher organisms, which makes it advantageous to study DCV function in C. elegans. The cell bodies of (cholinergic) motoneurons are located in the ventral nerve cord (VNC) and project to the dorsal site, where they innervate dorsal muscle cells (Figure II.14). In this way, the cell bodies, where DCVs are generated and their synapses, where DCVs are 
released, are clearly distinguishable from each other, allowing the study of DCV transport.

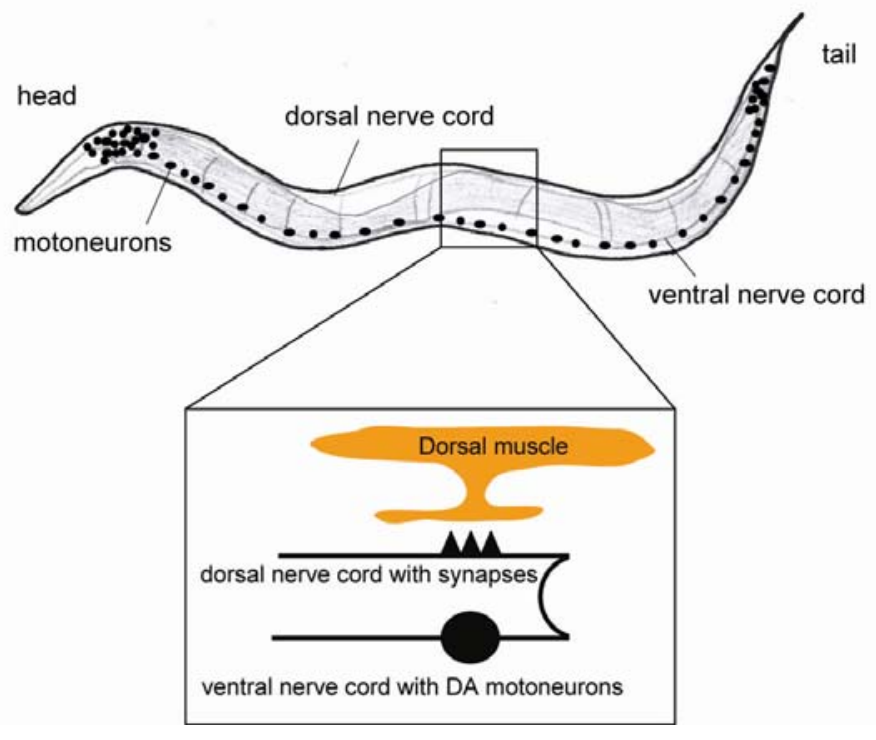

Figure II.14. Cholinergic motoneurons of the ventral nerve cord (VNC) project to the dorsal nerve cord (DNC) in C. elegans. This enables the follow of DCV trafficking from the neuronal cell body, the location where they are generated to the sites where they are released, at the DNC.

In order to analyze DCV function, we used an integrated C. elegans strain [nuIs183] stably expressing fluorescently-labeled proneuropeptide, NLP-21-VENUS, specifically in the cell bodies of DA and DB cholinergic motoneurons (Sieburth et al, 2007). NLP-21-VENUS is a fusion protein of the proneuropeptide NLP-21 with the yellow fluorescent protein VENUS. It has been shown that the proneuropeptides NLP-21VENUS is packaged into DCVs together with their processing enzymes (Figure II.15), transported to axons of the dorsal nerve cord (DNC), and released into the body cavity (Sieburth et al, 2007). Once secreted, NLP-21-derived VENUS is subsequently endocytosed by six macrophage-like scavenger cells (called coelomocytes), which constantly filter the body fluid by bulk endocytosis (Fares \& Greenwald, 2001a) (Figure II.16). 


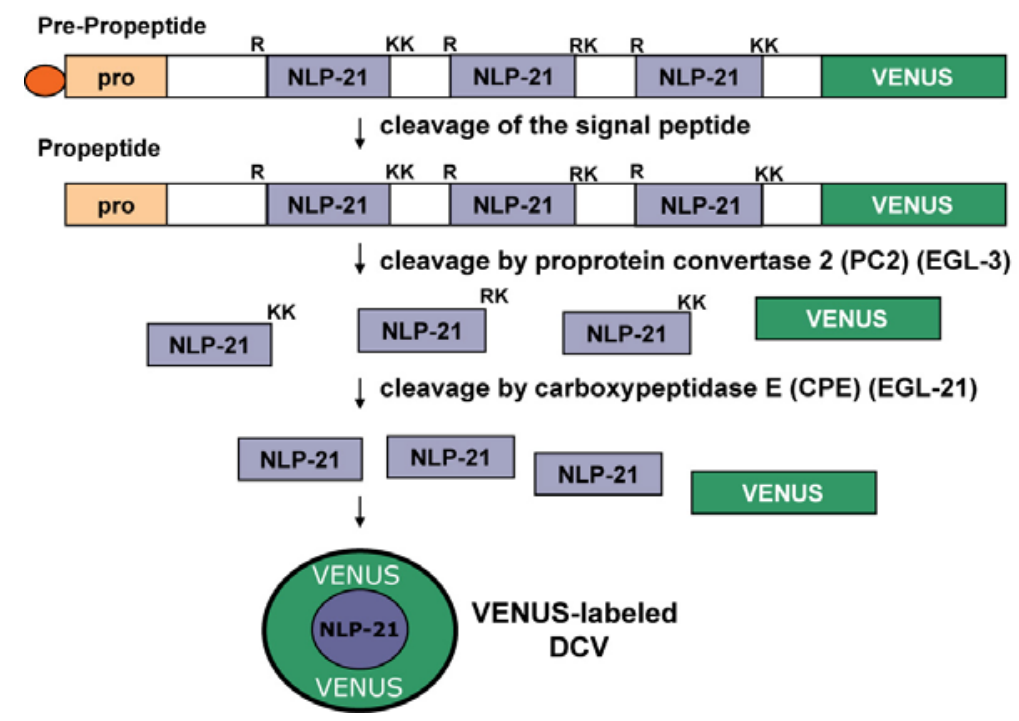

Figure II.15. DCVs can be labeled by expressing the proneuropeptide NLP-21 fused to VENUS in cholinergic motoneurons in C. elegans. This precursor protein is packaged together with its processing enzymes (EGL-3, EGL-21) into DCVs. After processing into bioactive neuropeptides NLP-21, peptides aggregate and form the dense-core, whereas VENUS is present in the soluble fraction of DCVs.

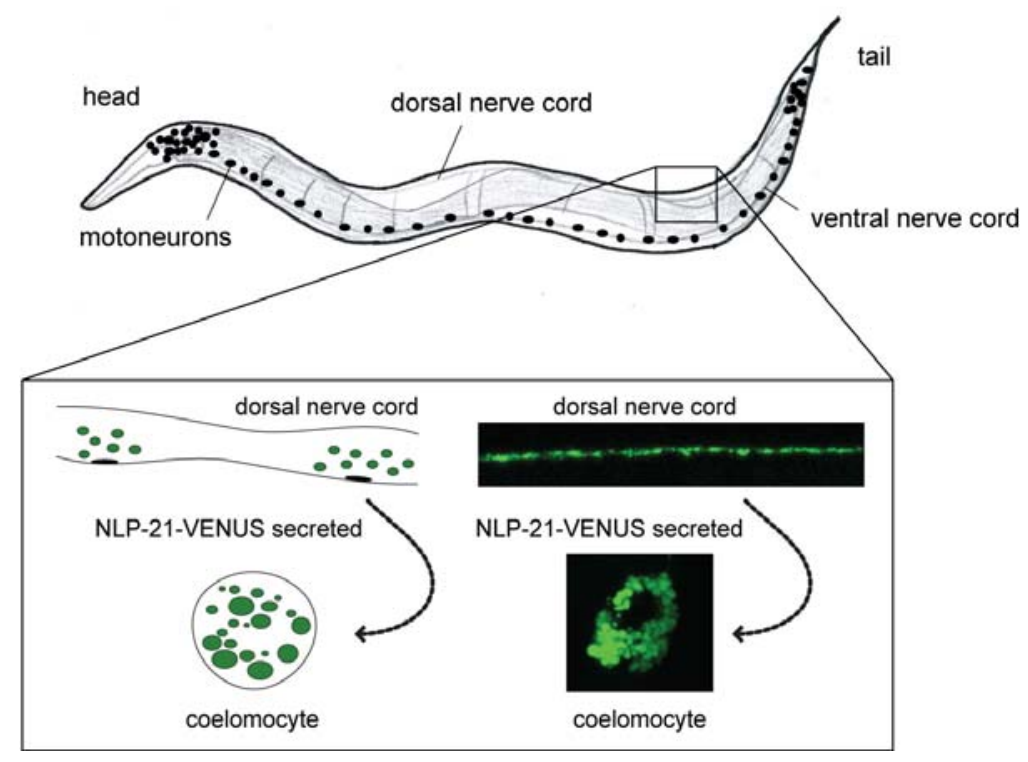

Figure II.16. NLP-21-VENUS assay to detect DCV trafficking and secretion defects. Schematic representation of DCV assay used in this study. The proneuropeptide NLP-21-VENUS fusion protein is expressed in dorsally projecting DA and DB cholinergic motoneurons. VENUS labeled DCVs are transported to the DNC where VENUS is secreted into the body cavity (Gracheva et al, 2007; Sieburth et al, 2007). Here, VENUS is taken up by coelomocytes of $C$. elegans (Fares \& Greenwald, 2001b). 
In this study, the DCV assay was utilized to identify further proteins involved in $\mathrm{DCV}$ biogenesis and maturation in C. elegans. This $\mathrm{PhD}$ thesis is separated into three chapters, which all cope with RAB-2-related DCV maturation in C. elegans. The first chapter focuses on the identification of a TBC-domain containing GAP, which was found to specifically regulate RAB-2 activity. The second and third chapters elucidate the functions of novel molecules, which were found to work alongside RAB-2 to regulate the biogenesis/maturation of DCVs. Individual aims are explained at the start of the respective chapters. 


\section{Chapter 1}

\section{Regulation of the small GTPase RAB-2 during dense-core vesicle maturation}

\section{Specific Introduction}

Rab GTPases are important regulators of membrane trafficking. They act as molecular switches. In their active, GTP-bound form, they initiate downstream events by recruitment of effector proteins to specialized membrane domains (Hutagalung \& Novick, 2011). The interaction between Rab GTPases and effector proteins is spatially and temporally tightly controlled by regulating proteins, guanine nucleotide exchange factors (GEFs) and GTPase activating proteins (GAPs). Whereas GEFs mediate activation of Rab GTPases, GAPs terminate the activity of Rab proteins (Stenmark, 2009) (Figure III.1). Therefore it is believed that GEFs control the location at which a Rab is activated and GAPs control the lifetime of the activated state of the Rab (Rybin et al, 1996; Stenmark, 2009). 


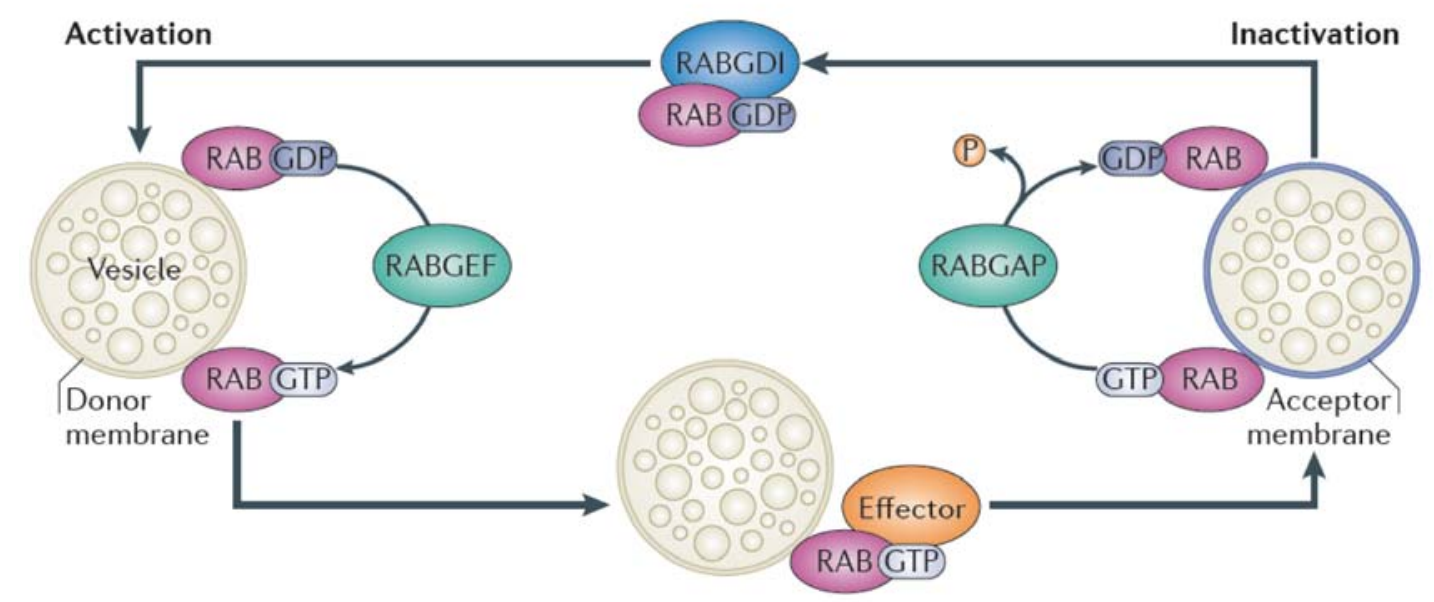

Figure III.1. The Rab cycle. The recruitment of inactive, GDP-bound Rab from the acceptor to the donor membranes is facilitated by the Rab-specific GDP dissociation inhibitor (RABGDI). The inactive Rab associates with the donor membrane through its prenylated C-terminus (not shown). A Rab-specific GEF (RABGEF) activates the Rab by accelerating the exchange of the bound GDP for GTP. Active, GTP-bound Rab interacts with effectors. Rab-specific GTPase activating proteins (RABGAPs) stimulate GTP hydrolysis, and GDP-bound Rab is delivered for the next cycle. Source: Reprinted by permission from Macmillan Publishers Ltd: [Nature Reviews Molecular Cell Biology] (Frasa et al, 2012), copyright 2012.

\section{III.1 The Rab GAP family: TBC-domain containing proteins}

Most of the mammalian Rab GAPs identified so far contain a conserved TBCdomain (Tre-2/Bub2/Cdc16), except for Rab3GAP (Fukui et al, 1997). The TBC-domain consists of approximately 200 amino acids and harbors the catalytic motif 'IxxDxxR' (Barr \& Lambright, 2010; Neuwald, 1997). It was shown that the TBC-domain alone was sufficient to conduct GAP activity in vitro (Albert et al, 1999).

In mammals about 44 TBC-domain containing proteins (referred as TBC proteins hereafter) were identified until now (Frasa et al, 2012), whereas over 60 Rab proteins (including splice variants) exists in humans (Pereira-Leal \& Seabra, 2000). This indicates either that additional non-TBC-domain containing GAPs may exist, or that multiple GAPs may exhibit activity towards more than one Rab. Interestingly, it was also shown that a single Rab can be regulated by two different GAPs, depending on its localization (Barr \& Lambright, 2010). The yeast Rab, called Ypt1p, is inactivated by Gyp8p (్AP 
for $\underline{\text { Ypt) }}$ at the ER membranes (De Antoni et al, 2002; Haas et al, 2007), whereas Gyp1p has GAP activity towards Ypt1p at the Golgi complex (De Antoni et al, 2002; Du \& Novick, 2001). This suggests that Ypt1p is required for different membrane trafficking steps and is, therefore, controlled by distinct GAPs localized at specific sites (Barr \& Lambright, 2010).

Noteworthy to mention is the fact that Rab proteins can also bind to Rab GAPs, even though they are not substrates for these GAPs (Fukuda, 2011). This interaction normally occurs via a domain outside of the TBC-domain: The Rab GAP, EVI5, binds to Rab10 via a coiled-coil-domain (Fukuda et al, 2008) and GAPCENA binds to Rab36 via its PTB-domain (Kanno et al, 2010) (see also Figure III.3). This suggests that TBCdomain Rab GAPs can also be Rab effectors at the same time. Thus, several Rab GAPs might be recruited by a specific Rab GTPase to inactivate the Rab from a previous trafficking step or exclude another Rab GTPase from its domain. Through these mechanisms directionality during membrane tracking and sorting can be achieved (Rivera-Molina \& Novick, 2009).

\section{III.2 Mode of action of Rab GAPs by a dual-finger mechanism}

Several signature motifs within TBC-domains have been identified for Rab GAPs (Neuwald, 1997). These sequences include the motifs "IxxDxxR" and "YxQ" (whereby $x$ can be any amino acid) (Neuwald, 1997). Mutational analysis of the "IxxDxxR" motif defined the catalytic "arginine finger", which is also essential for GAPs of Ras and Rho GTPases (Albert et al, 1999; Du \& Novick, 2001). The crucial role of the arginine was further confirmed by the crystal structure analysis of the GAP Gyp1p in complex with Rab33 and the transition state mimic $\mathrm{AlF}_{3}$ (Pan et al, 2006) (Figure III.2A). It revealed that the arginine inserts into the nucleotide site to stabilize the GDP- $\mathrm{AlF}_{3}-\mathrm{H}_{2} \mathrm{O}$ transition state by mediating hydrogen bonding interactions with GDP and $\mathrm{AlF}_{3}$ (Barr \& Lambright, 2010; Pan et al, 2006) (Figure III.2B). Furthermore, the glutamine in the DxxGQ motif of Rab GTPases engages the backbone of the TBC-domain, allowing the glutamine finger of 
the "YxQ" motif to mediate polar interaction with the $\mathrm{AlF}_{3}-\mathrm{H}_{2} \mathrm{O}$ moiety (Pan et al, 2006). Since both arginine and glutamine fingers are essential for GAP activity, the mode of action of Rab GAPs is referred to as “dual-finger mechanism” (Pan et al, 2006).

There is one Rab GAP identified so far, which does not possess a TBC-domain, Rab3GAP. It shows specificity towards the small GTPase Rab3 (Fukui et al, 1997; Nagano et al, 1998). However, it is believed that it might also act on other GTPases of the Ras superfamily, since plants have a Rab3GAP homolog, but lack Rab3 (Barr \& Lambright, 2010). Although, there is no crystallization data available for Rab3GAP, it is believed that this GAP uses a similar arginine-finger catalytic mechanism to conduct GAP activity that is described above (Clabecq et al, 2000).

A

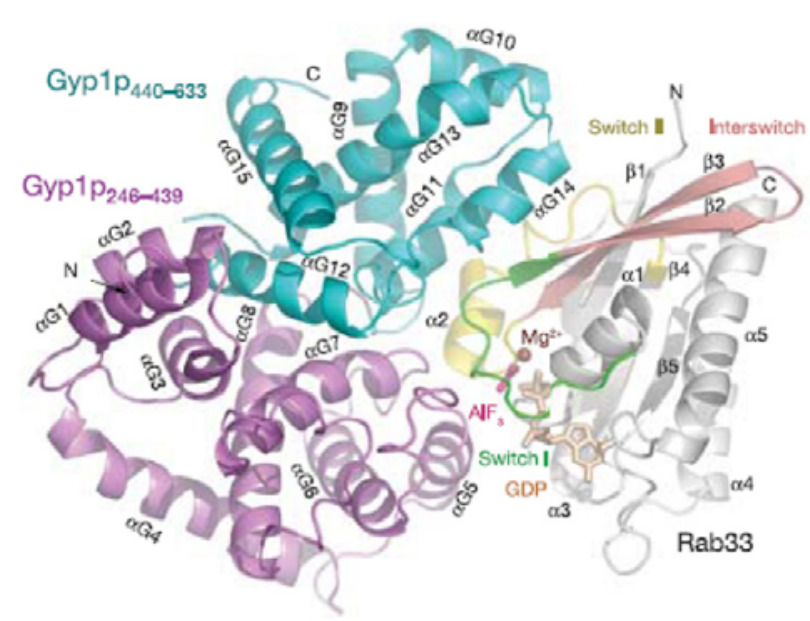

B

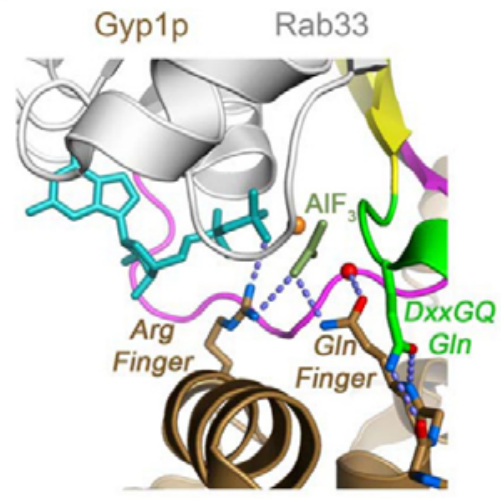

Figure III.2. Protein structure of the transition state of the TBC-GAP, Gyp1p, in complex with Rab33-GDP-AlF 3 . (A) Ribbon representation of the complex with the TBC-domain of Gyp1p and functional regions of Rab33. Source: Reprinted by permission from Macmillan Publishers Ltd. [Nature] (Pan et al, 2006), copyright 2006. (B) Enlargement into the nucleotide site of the complex with GDP and $\mathrm{AlF}_{3}$. Source: Reprinted from (Barr \& Lambright, 2010), with permission from Elsevier.

\section{III.3 Functions of Rab GAPs}

It has been shown that Rab GAPs are not only negative regulators of Rab proteins, but also regulate cellular processes such as autophagy (Behrends et al, 2010; Itoh et al, 2011), cytokinesis (Faitar et al, 2005), and macropinocytosis (Frittoli et al, 2008) by 
integrating signaling between different Rabs or between Rabs and other small GTPases (Frasa et al, 2012). Therefore, TBC Rab GAPs might be required for the crosstalk between different small GTPase families during intracellular trafficking (Frasa et al, 2012). For a detailed list of identified functions of each mammalian TBC GAP, see review (Frasa et al, 2012). Furthermore, an emerging role of Rab GAPs in diseases has been shown (Frasa et al, 2012; Hutagalung \& Novick, 2011). Mutations in the Rab3GAP lead to neurodevelopmental and ophthalmological phenotypes summarized as the Warburg Micro and Martsolf syndromes (Aligianis et al, 2005; Aligianis et al, 2006).

\section{III.4 Regulation of GAPs}

When the first Rab GAPs were identified, it was surprising that many GAPs showed little substrate specificity in vitro (Albert \& Gallwitz, 1999; Albert et al, 1999). It was difficult to assign a single GAP to a specific trafficking pathway in vitro, because it was often the case that an individual GAP would stimulate Rabs functioning in opposing pathways (Albert et al, 1999). This indicated that specificity of GAPs towards their Rab proteins had to be further regulated in vivo, either by their subcellular localization or by additional regulatory mechanism like phosphorylation (Barr \& Lambright, 2010). The finding that functional fragments of TBC-domains were more active than their full-length proteins further supported the idea that these GAPs harbor regions important for regulating activity through interactions with other proteins (Albert et al, 1999).

In summary, Rab GAPs can be regulated by various mechanisms: alteration of their localization, auto-inhibition or regulation of catalytic activity (Frasa et al, 2012). These mechanisms can be accomplished by direct protein-protein interactions that can be mediated by additional protein domains within Rab GAPs (Frasa et al, 2012; Fukuda, 2011) (Figure III.3). For a list of all detectable protein domains in C. elegans Rab GAPs, see Table III.1 and Figure III.4. Interestingly, for one of these domains [RUN-(RPIP8, UNC-14, and NESCA) domain], it has been shown that they are required for interaction with other small GTPases (Callebaut et al, 2001; Yang et al, 2007). 

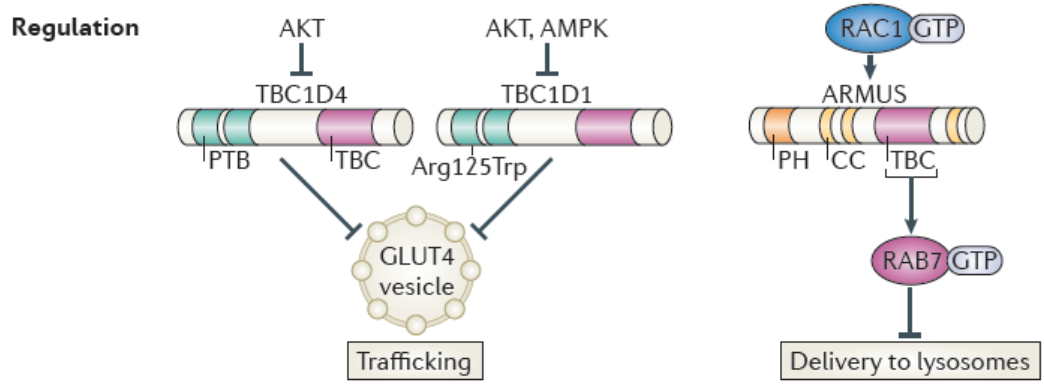

Localization
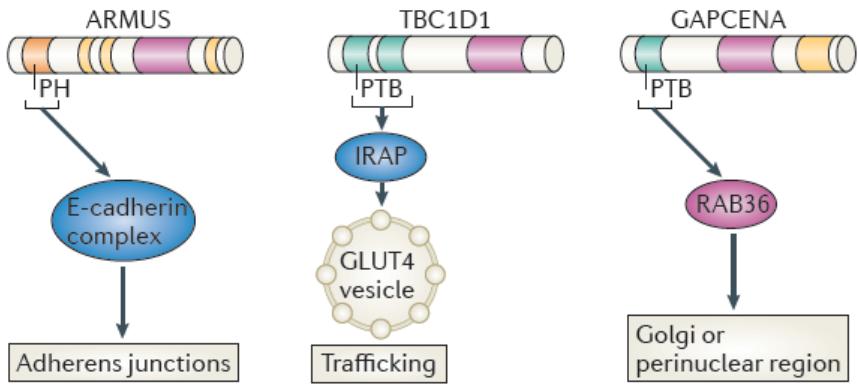

Figure III.3. Mechanisms to regulate RAB GAP activity or localization. Phosphorylation of TBC1D4 and TBC1D1 leads to their inactivation, which consequently allows for the translocation of GLUT4-containing vesicles. The Rab GAP, ARMUS, is activated by binding to Rac1, which in turn inactivates Rab7 and regulates the transport of cadherin-containing cargos to lysosomes. The localization of RAB GAPs to different intracellular compartments can be regulated by other domains: PH-domains (ARMUS) or PTB domains (TBC1D1, GAPCENA). Note, interaction of GAPCENA with Rab36 is GTP independent, thus Rab36 might be an effector rather than a substrate. AMPK, AMP-activated protein kinase; CC, coiled-coil; GLUT4, glucose transporter 4; IRAP, insulin-regulated aminopeptidase; $\mathrm{PH}$, pleckstrin homology; PTB, phosphotyrosinebinding. Source: Reprinted by permission from Macmillan Publishers Ltd: [Nature Reviews Molecular Cell Biology] (Frasa et al, 2012), copyright 2012.

\section{III.5 TBC-domain containing proteins in C. elegans}

Utilizing knockout animals is a great tool to analyze protein function in vivo. However, no TBC knockout (KO) mice have been reported so far [whereas KO mice exist for the catalytic domain p130 of the Rab3GAP, which does not possess a TBCdomain (Sakane et al, 2006)]. Contrary to this, more than a half of all tbc-genes in $C$. elegans mutant alleles exist (www.wormbase.org) (Table III.1). Furthermore, knockdown of gene expression by RNAi is a well-established technique in C. elegans (Xu \& Kim, 2011). These readily available genetic tools make it reasonable to study the function of TBC-containing RAB GAP in C. elegans. 
Table III.1. List of $C$. elegans tbc genes and available mutant alleles.

\begin{tabular}{|c|c|c|c|}
\hline $\begin{array}{l}\text { Gene / } \\
\text { sequence name }\end{array}$ & Alleles & $\begin{array}{l}\text { Expression pattern } \\
\text { (wWw.wormbase.org) }\end{array}$ & $\begin{array}{l}\text { Additional } \\
\text { domains }\end{array}$ \\
\hline$t b c-1 / F 20 D 1.2$ & $t m 2282$ & n.a. & RHOD-domain \\
\hline$t b c-2 / Z K 1248.10$ & $\begin{array}{l}q \times 20 \\
\operatorname{tm} 2241\end{array}$ & $\begin{array}{l}\text { Ubiquitously expression in the embryo, expression } \\
\text { in pharyngeal muscle, hypodermis and intestine, } \\
\text { gonadal sheath cells, neuronal expression }\end{array}$ & $\begin{array}{l}\text { PH-domain } \\
\text { CC domain }\end{array}$ \\
\hline$t b c-3 / F 32 B 6.8$ & $x h 23$ & $\begin{array}{l}\text { Expression in pharynx, several unidentified } \\
\text { neurons in the head, distal tip cell, seam cell, and } \\
\text { several cells in the tail region }\end{array}$ & \\
\hline$t b c-4 / T 24 D 11.1$ & $\begin{array}{l}\text { tm3255 } \\
\text { ok2928 } \\
\text { ok3041 }\end{array}$ & $\begin{array}{l}\text { Expression in pharynx, pharyngeal-intestinal } \\
\text { valve, nervous system }\end{array}$ & CC domain \\
\hline$t b c-5 / r b g-3 / B 0393.2$ & $\operatorname{tm} 1910$ & $\begin{array}{l}\text { Specific expression in amphid neurons and } \\
\text { phasmid neurons }\end{array}$ & \\
\hline$t b c-6 / C 04 A 2.1$ & n.a. & n.a. & \\
\hline$t b c-7 / C 31 H 2.1$ & $\begin{array}{l}\text { tm1856 } \\
\text { (lethal) }\end{array}$ & n.a. & TLDc-domain \\
\hline$t b c-8 / C 38 H 2.1$ & tm3802 & Neuronal expression & RUN domain \\
\hline$t b c-9 / Y 45 F 10 \mathrm{~A} .6$ & n.a. & Expression in pharynx and intestine & $\begin{array}{l}\text { GRAM domain } \\
\text { CC domain }\end{array}$ \\
\hline$t b c-10 / R 06 B 10.5$ & n.a. & $\begin{array}{l}\text { Expression in pharynx, intestine, reproductive } \\
\text { system, vulval muscle, hypodermis, nervous } \\
\text { system }\end{array}$ & \\
\hline$t b c-11 / F 35 H 12.2$ & ok2576 & $\begin{array}{l}\text { Expression in pharynx, intestine, reproductive } \\
\text { system, uterus, spermatheca, uterine valve, } \\
\text { nervous system }\end{array}$ & $\begin{array}{l}\text { PTB-domain } \\
\text { CC domain }\end{array}$ \\
\hline$t b c-12 / R 11 B 5.1$ & $g k 362$ & n.a. & \\
\hline tbc-13/F45E6.3 & ok1812 & Expression in intestine, hypodermis & \\
\hline tbc-14/E01G4.1 & n.a. & n.a. & \\
\hline$t b c-15 / Y 48 E 1 C .3$ & n.a. & n.a. & \\
\hline$t b c-16 / F 49 E 10.1$ & n.a. & Expression in nervous system & \\
\hline tbc-17/Y46G5A.1 & n.a. & n.a. & \\
\hline tbc-18/F41D9.1 & ok2374 & Expression in nervous system. & $\begin{array}{l}\text { SH3-domain } \\
\text { RUN-domain }\end{array}$ \\
\hline$t b c-19 / C 31 E 10.8$ & n.a. & n.a. & PH domain \\
\hline$t b c-20 / Y 54 E 2 A .12$ & n.a. & n.a. & TM domain \\
\hline tbck-1/C33F10.2 & $\begin{array}{l}\text { ok2038 } \\
\text { ok2064 }\end{array}$ & n.a. & STYKc domain \\
\hline
\end{tabular}

Available expression pattern of $t b c$ genes are given. Some of TBC-domain containing proteins also possess additional domains, which are listed. For abbreviations see Figure legend III.5. n.a., not available. Note $t b c-14$ and $t b c-15$ might be one gene. RHOD-domain of TBC-1 is predicted to be inactive. For further details, see www.wormbase.org. Abbreviations: CC, coiled-coil domain; RHOD, rhodanese homology domain; SH3, Src homology domain 3; STYKc, protein kinase domain of undetermined specificity; TLDc, TBC and LysM domain-containing; TM, transmembrane; TBC, Tre-2/Bub2/Cdc16; PH, Pleckstrin homology; RUN, RPIP8, UNC-14, and NESCA; PTB, phosphotyrosine-binding domain; GRAM, Glucosyltransferases, Rab-like GTPase activators and Myotubularins. 
In C. elegans, 20 TBC-domain containing proteins have been identified (Table III.1) and almost half of these have homologs in humans (Fukuda, 2011) (Figure III.4).

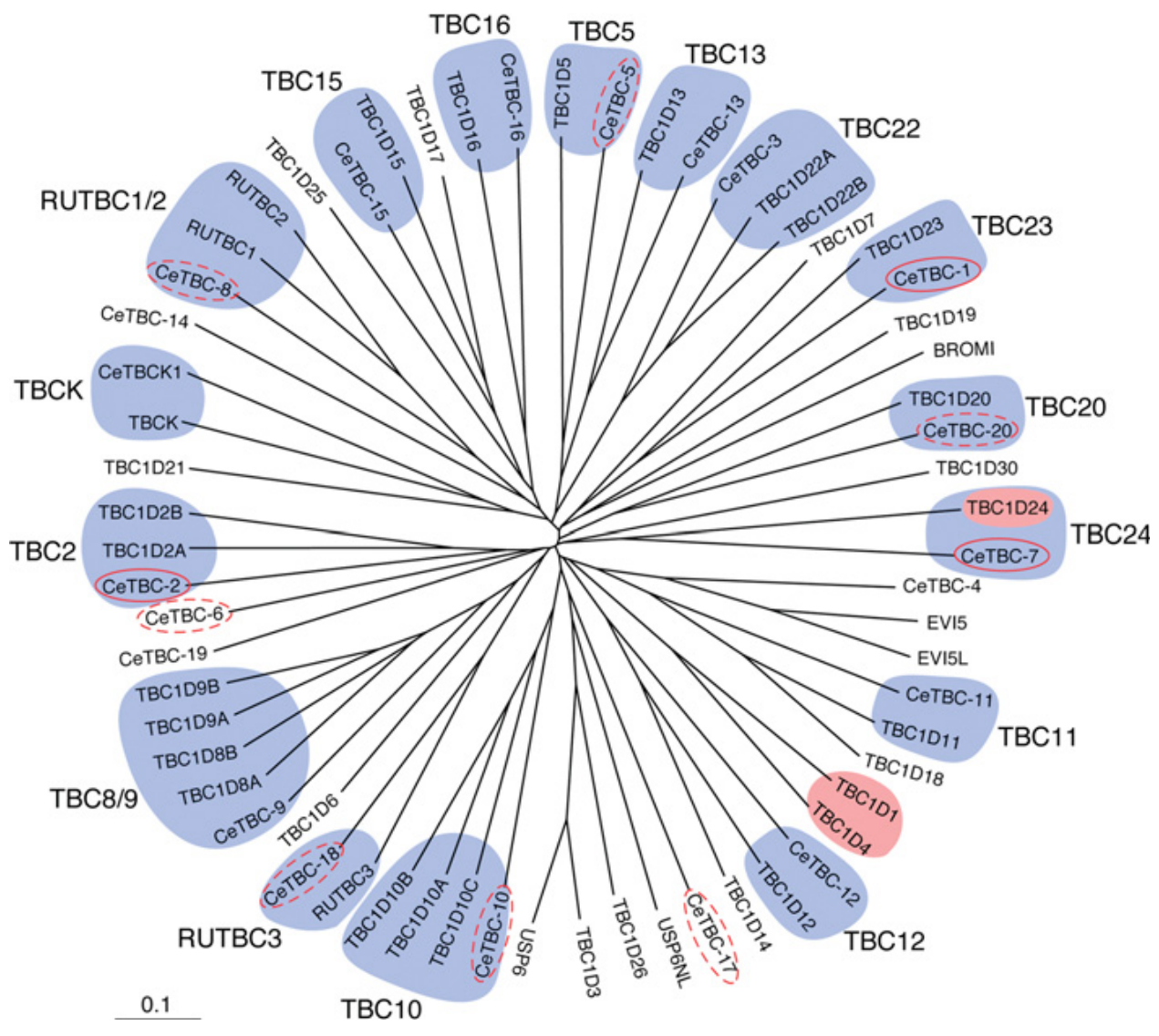

Figure III.4. Molecular dendrogram of human (Homo sapiens) and nematode (C. elegans) TBC proteins. TBC proteins that are conserved between humans and C. elegans are shown in blue. Human TBC proteins associated with diseases are highlighted by a red background, and solid red circles and broken red circles indicate nematode TBC proteins, whose deficiency causes lethality and certain abnormalities respectively (www.wormbase.org). Ce, C. elegans. Source: reproduced with permission from (Fukuda, 2011), (c) the Biochemical Society.

Protein structures of all predicted C. elegans TBC-proteins are shown in Figure III.5. TBC-2 and TBC-5 are the only TBC-proteins in C. elegans that have been described so far (Chotard et al, 2010; Mukhopadhyay et al, 2005). It could be shown that TBC-2 is a putative RAB-5 GAP (Chotard et al, 2010). 


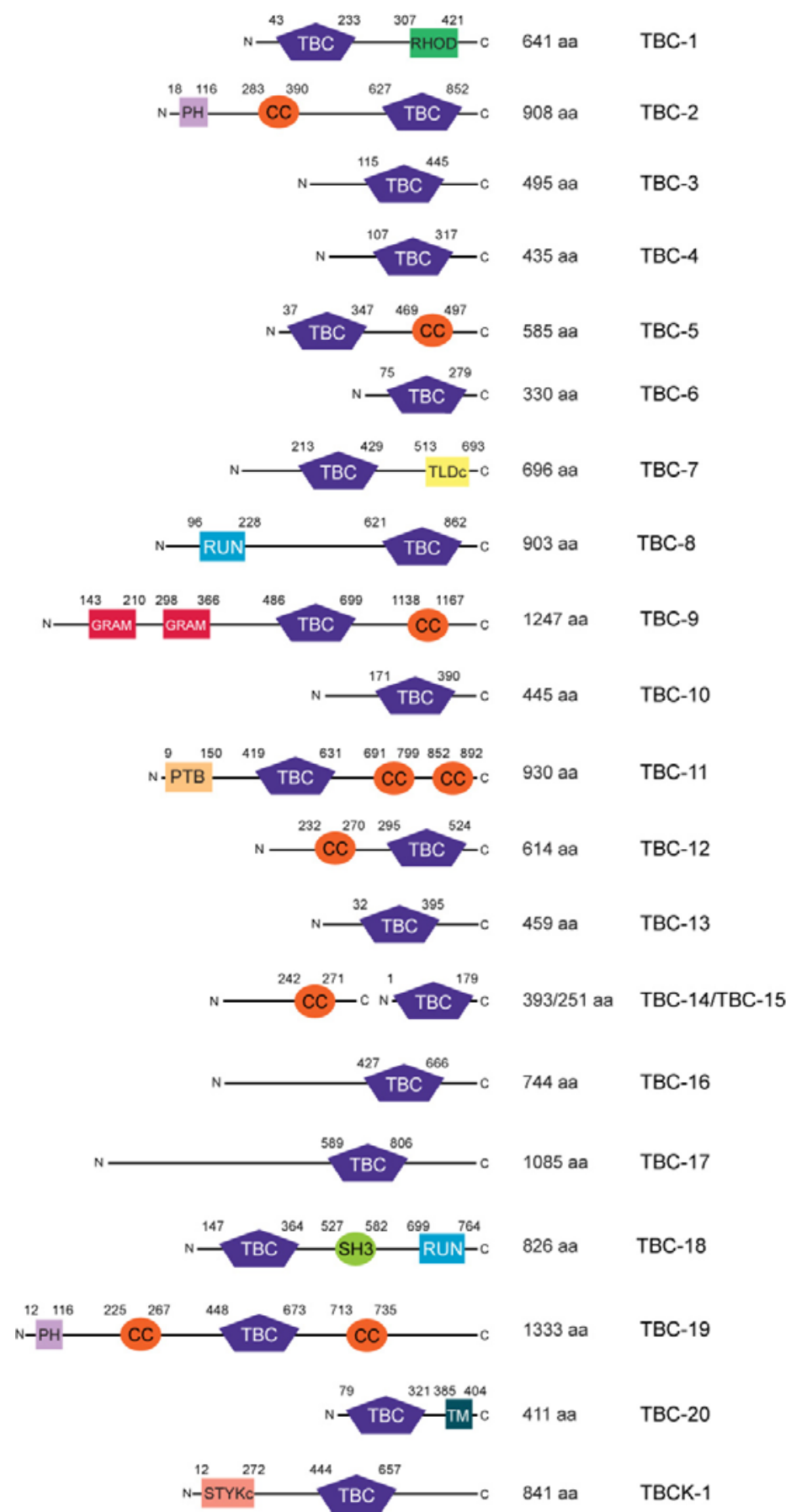

Figure III.5. Structure of TBC-domain containing proteins in C. elegans. Protein domains of C. elegans TBC-domain containing proteins were identified by using the SMART (Simple Modular Architecture Research Tool) program (http://smart.embl-heidelberg.de/). Protein and domain sizes are indicated. Abbreviations: CC, coiled-coil domain; RHOD, rhodanese homology domain; SH3, Src homology domain 3; STYKc, protein kinase domain of undetermined specificity; TLDc, TBC and LysM domain-containing; TM, transmembrane; TBC, Tre2/Bub2/Cdc16; PH, Pleckstrin homology; RUN, RPIP8, UNC-14, and NESCA; PTB, phosphotyrosine-binding domain; GRAM, Glucosyltransferases, Rab-like GTPase activators and Myotubularins. Note: gene product of $t b c-14$ and $t b c-15$ might be one protein. 


\section{III.6 Aims of this study}

DCV biogenesis is an important and highly regulated mechanism in neuronal and endocrine cells. An established DCV assay allowed the identification of various protein families, which are implicated in the biogenesis and regulation of DCVs. In this assay the fluorescence levels of the transported VENUS cargo derived from the neuropeptide NLP21 at its axonal release sites in the DNC in C. elegans can be measured. By utilizing this assay the small GTPase RAB-2 was identified to be involved in DCV maturation (Edwards et al, 2009; Sumakovic et al, 2009). There is accumulating evidence that a cycling of RAB-2 between a GDP-bound and a GTP-bound state is especially required for DCV maturation (Sumakovic et al, 2009). Due to the low intrinsic nucleotide exchange and hydrolysis rate of Rab2 (Sumakovic et al, 2009), it is obvious that accessory regulatory proteins such as GTPase hydrolyzing proteins (GAPs) and guanine nucleotide exchange factors (GEFs) must exist that regulate Rab2 function during DCV maturation. Therefore, we attempted to identify regulators for RAB-2, which are also involved in DCV maturation in C. elegans. After the identification of a putative (TBCdomain containing) RAB-2 GAP, we studied its role in DCV maturation in cholinergic motoneurons and its function in other non-neuronal tissues. We observed similarities and differences in phenotypes between mutants of RAB-2 and the GAP of interest indicating the presence of additional GAPs in neurons and other tissues.

This study was performed with the help of other members of the lab: Electron microscopy (EM) analysis was performed by Dr. Jan Hegermann and protein expression tests in bacteria were conducted by Dr. Sabine König. The "protein expression and purification core facility” at the EMBL, Heidelberg supported us with the protein expression in insect cells. 


\section{Chapter 1}

\section{Results}

\section{IV.1 tbc-8 mutants display DCV maturation defects}

To identify the Rab GAP that regulates RAB-2 during neuronal DCV maturation, we systematically tested mutants of TBC-domain containing GAPs in $C$. elegans. We concentrated on $t b c$ genes that are predicted to be expressed in neurons and on those where mutants were available (www.wormbase.org; Table III.1). These $t b c$ mutants were analyzed for trafficking defects of the DCV marker NLP-21VENUS (Figure IV.1).

\section{NLP-21-VENUS in dorsal nerve cord}

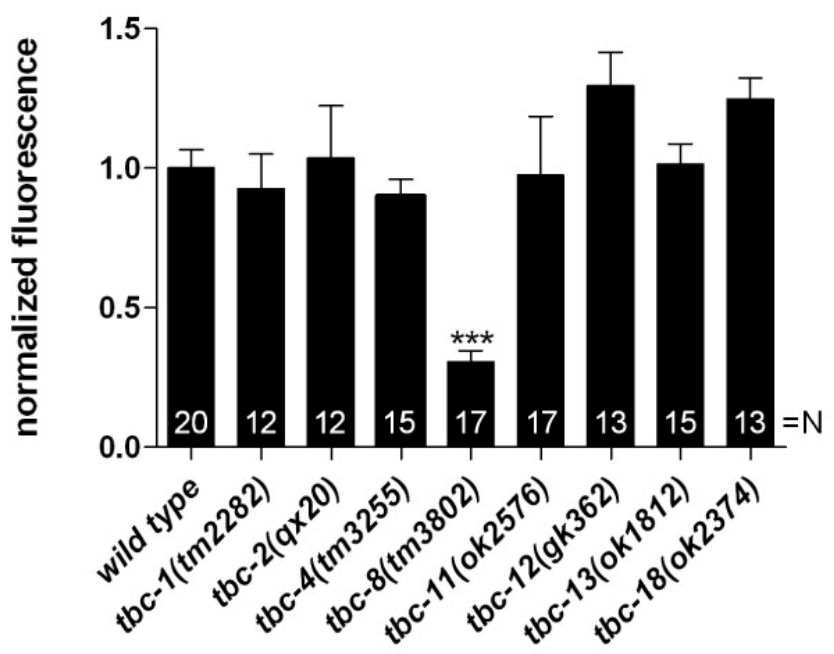

Figure IV.1. Analysis of mutants of TBC-domain containing GAPs in C. elegans for DCV trafficking defects of the NLP-21-VENUS marker. This assay revealed that from the set of mutants analyzed only $t b c-8(t m 3802)$ deletion mutants displayed decreased fluorescence levels of VENUS derived from NLP-21 in the dorsal nerve cord similar to unc108/rab-2 mutants (Figure IV.7). Error bars $=$ s.e.m. (***, P < 0.0001; ANOVA with Bonferroni post test). Source: (Hannemann et al, 2012). 
In this screen, only $t b c-8(t m 3802)$ deletion mutants displayed decreased NLP21-derived VENUS levels in the DNC axons, similar to unc-108/rab-2 mutants (Edwards et al, 2009; Sumakovic et al, 2009) (Figure IV.1; IV.8). Interestingly, all other tested $t b c$ mutants showed wild type like or slightly (non-significantly) increased VENUS fluorescence in the DNC axons (Figure IV.1). Inactivation of TBC8 led to a $74.95 \pm 3.51 \%$ decrease of NLP-21-derived VENUS fluorescence in axons compared to wild type (Figure IV.2).

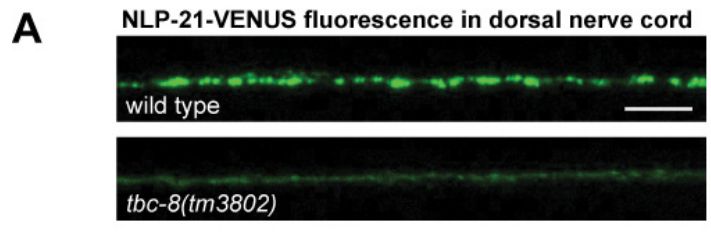

NLP-21-VENUS in dorsal nerve cord

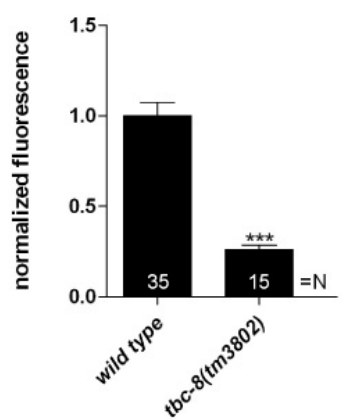

B NLP-21-VENUS fluorescence in coelomocytes
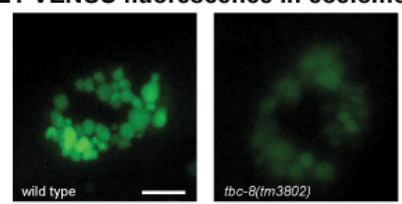

NLP-21-VENUS

in coelomocytes

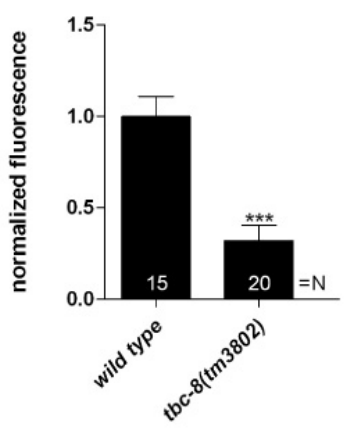

Figure IV.2. $t b c-8(t m 3802)$ mutants show decreased NLP-21-derived VENUS levels in the dorsal nerve cord and coelomocytes. Inactivation of TBC-8 led to a $74.95 \pm 3.51 \%$ and $68.17 \pm 6.94 \%$ decrease of NLP-21-derived VENUS fluorescence in (A) axons and (B) coelomocytes, respectively, compared to wild type. Scale bar represents $5 \mu \mathrm{m}$. Error bars = s.e.m. (***, P < 0.0001; Student's t-test). Source: (Hannemann et al, 2012).

Currently, there is no other mutant $t b c-8$ allele available besides the deletion mutant $t b c-8(t m 3802)$. The databank "Wormbase” (www.wormbase.org) lists another allele, $t b c-8(g k 2897)$, which harbours a silent mutation. Therefore, we did not include this allele in this study. However, to independently test whether TBC-8 is involved in DCV maturation, we analyzed tbc-8 knockdown by RNAi in C. elegans. Downregulation of $t b c-8$ expression led to a similar DCV trafficking defect as seen in tbc-8(tm3802) deletion mutants (Figure IV.3). 

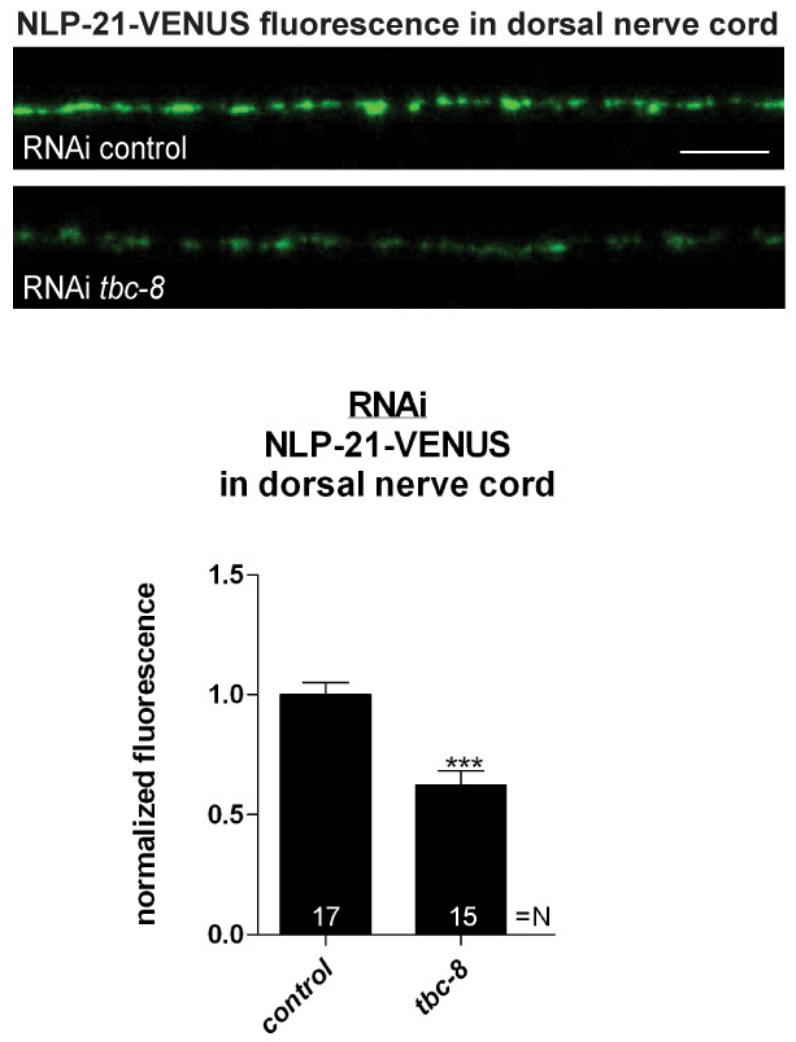

Figure IV.3. Downregulation of $t b c-8$ expression leads to decreased fluorescence levels of VENUS derived from NLP-21 in the dorsal nerve cord. RNAi of $t b c-8$ expression in an eri-1(mg366); nuIs183 background caused a decreased VENUS fluorescence level in the dorsal nerve cord by $37.67 \pm 5.80 \%$ compared to the control strain. Control: The mock vector (L4440) was used. Similar results were observed in $t b c-8(t m 3802)$ deletion mutants (Figure IV.2). Scale bar represents $5 \mu \mathrm{m}$. Error bars $=$ s.e.m. (***, $\mathrm{P}<0.0001$; Student's t-test). Source: (Hannemann et al, 2012).

Similar to the decreased fluorescence levels of VENUS in the DNC of $t b c-$ 8(tm3802) mutants, the accumulation of secreted VENUS in coelomocytes was accordingly decreased by $68.17 \pm 6.94 \%$ (Figure IV.4) suggesting that less VENUS was secreted from the DNC. To determine whether the observed DCV phenotype was caused by TBC-8 malfunction in the nervous system and is, therefore, cellautonomous, we expressed $t b c-8$ cDNA pan-neuronally under the control of the $r a b-3$ promoter. Both, pan-neuronal expression of $t b c-8$ as well as expression of $t b c-8$ under the DA and DB cholinergic motoneuron specific unc-129 promoter (2641 bp promoter fragment upstream of the start ATG of unc-129) was able to rescue the NLP-21derived VENUS fluorescence levels in DNC and in coelomocytes (Figure IV.4). This demonstrated that TBC-8 is cell-autonomously required in cholinergic motoneurons for proper DCV trafficking. Furthermore, the catalytic GAP activity of TBC-8 was 
also shown to be crucial for DCV trafficking as expression of a catalytically inactive TBC-8 (R697A) was unable to rescue the NLP-21-VENUS trafficking phenotype (Figure IV.4) (Pan et al, 2006). This result highlights that TBC-8 acts as a RAB GAP required for effective DCV trafficking.

A

NLP-21-VENUS fluorescence in dorsal nerve cord
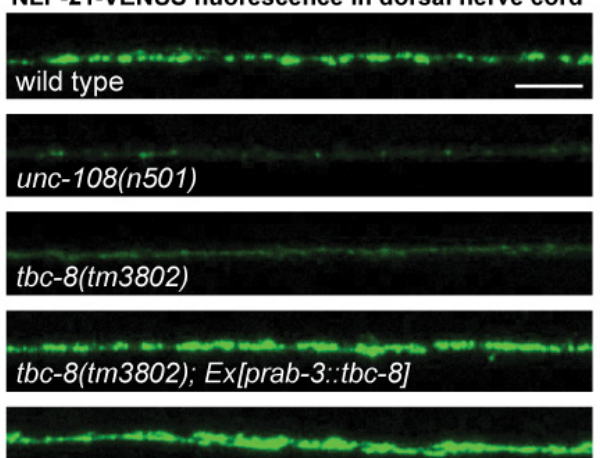
tbc-8(tm3802); Ex[punc-129::tbc-8]

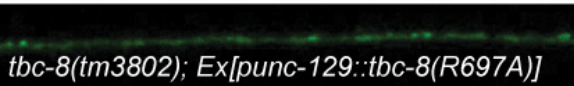

B

NLP-21-VENUS fluorescence in coelomocytes

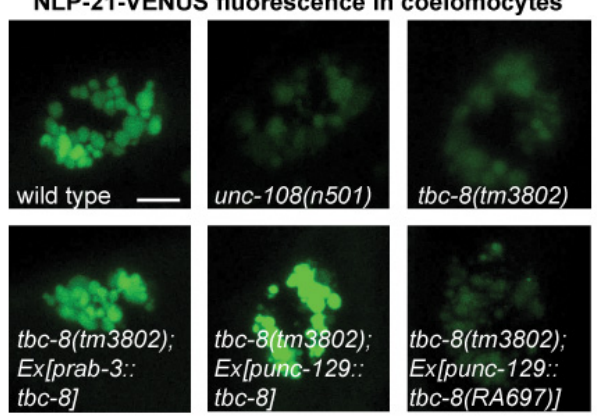

NLP-21-VENUS in dorsal nerve cord

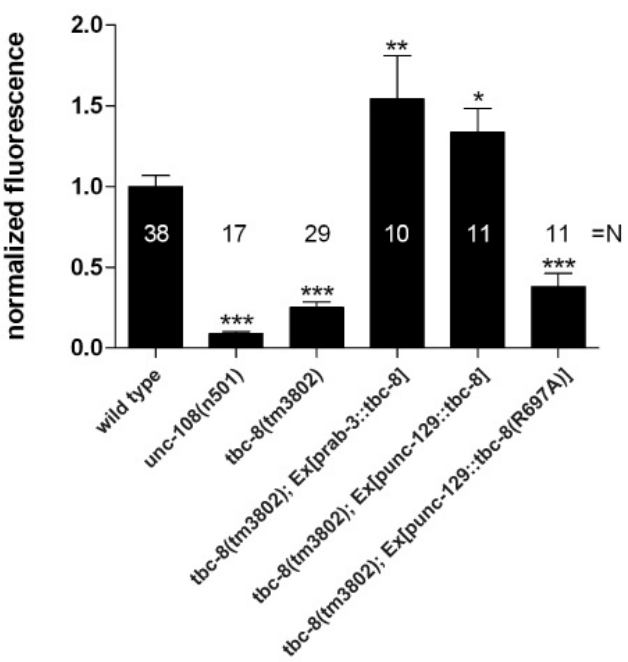

NLP-21-VENUS in coelomocytes

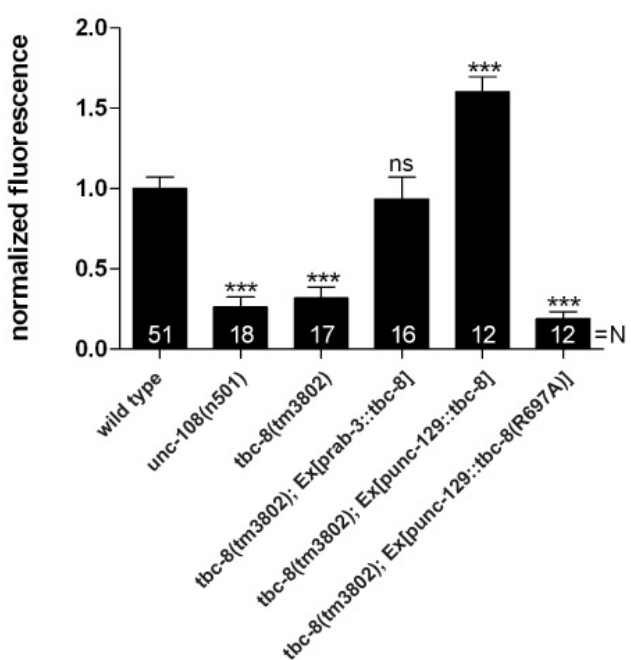

Figure IV.4. $t b c-8(t m 3802)$ showed decreased NLP-21-derived VENUS levels in the DNC (A) and in the coelomocytes (B), similar to unc-108/rab-2(n501) mutants. NLP-21-derived VENUS levels were rescued by expressing tbc-8 pan-neuronally (rab-3 promoter) as well as specifically in DA and DB cholinergic motoneurons (unc-129 promoter) demonstrating that TBC-8 acts cell autonomously. Strikingly, the catalytically inactive TBC-8 (R697A) (Pan et al, 2006) protein was not able to rescue the DCV phenotype, emphasizing that its GAP activity is responsible for decreased NLP-21-derived VENUS levels at the axons and in coelomocytes. Scale bars in DNC and coelomocytes represent $5 \mu \mathrm{m}$. Error bars $=$ s.e.m. ${ }^{* * *}$, $\mathrm{P}<0.0001$; **, $\mathrm{P}<0.001$; *, $\mathrm{P}<0.05$; Student's t-test). Source: (Hannemann et al, 2012). 
Previously, the role of RAB-2 in DCV maturation was examined (Edwards et al, 2009; Sumakovic et al, 2009). It was shown that in unc-108/rab-2 mutants NLP21-VENUS-derived cargo is partially lost in the endosomal-lysosomal degradation route during DCV maturation and therefore, less cargo is packaged into mature DCVs (Edwards et al, 2009; Sumakovic et al, 2009). These trafficking defects resulted in aberrant NLP-21-derived VENUS accumulation in the cell bodies of unc-108(n501) mutants. In order to test whether tbc-8(tm3802) mutants displayed the same trafficking defects as $u n c-108 / \mathrm{rab}-2$ mutants, we analyzed the size distribution of VENUS positive vesicles in the cell bodies. Similar to unc-108(n501) mutants, tbc8(tm3802) deletion mutant animals contained more of these large VENUS positive vesicles in the cell bodies of the VNC, whereas smaller vesicles filled with NLP-21derived VENUS are less present compared to wild type animals (Figure IV.5). These results indicate that $t b c-8(t m 3802)$ mutants have comparable DCV trafficking defects like unc-108/rab-2 mutants.

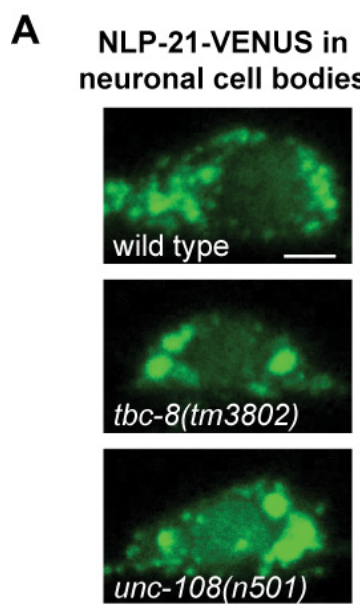

B cell bodies in ventral nerve cord

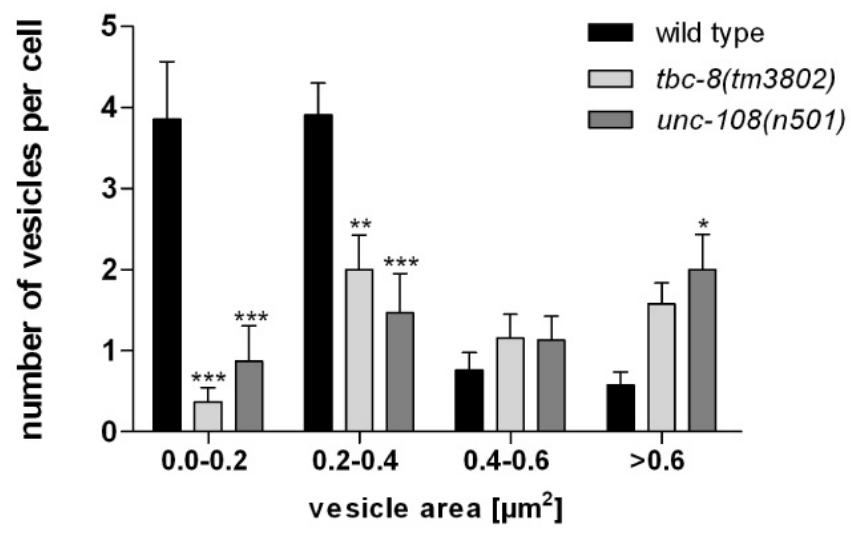

Figure IV.5. $t b c-8(t m 3802)$ mutants show similar DCV trafficking defects as unc108/rab-2(n501) mutants. (A) Representative pictures of NLP-21-derived VENUS fluorescence in the cell bodies of cholinergic motoneurons in the ventral nerve cord of wild type worms, $t b c-8(t m 3802)$ and unc-108(n501) mutants are shown. Scale bar represents $2 \mu \mathrm{m}$. Size distribution of VENUS positive vesicles in these mutants were analyzed in (B). Error bars $=$ s.e.m $(\mathrm{N}=15-21) .(* * *, \mathrm{P}<0.0001 ; * *, \mathrm{P}>0.001$, ANOVA with Bonferroni post test). Source: (Hannemann et al, 2012). 
To determine whether neuropeptides other than NLP-21 are also affected in $t b c-8(t m 3802)$, we tested the fluorescence levels of FMRFamide related peptide FLP3-derived VENUS and the insulin-like neuropeptide INS-22-VENUS in the DNC (Figure IV.6). FLP-3-derived VENUS levels in the DNC of $t b c-8$ mutants were decreased by $87.90 \pm 1.19 \%$, suggesting that VENUS derived from other neuropeptides was also lost (Figure IV.6A). However, analysis of INS-22-derived VENUS fluorescence levels was unchanged in tbc-8(tm3802), like in unc-108/rab-2 mutants (Edwards et al, 2009; Sumakovic et al, 2009) (Figure IV.6B).

A

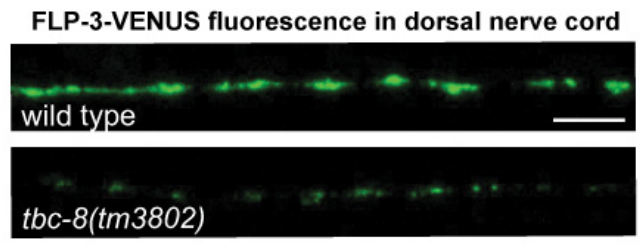

FLP-3-VENUS in dorsal nerve cord

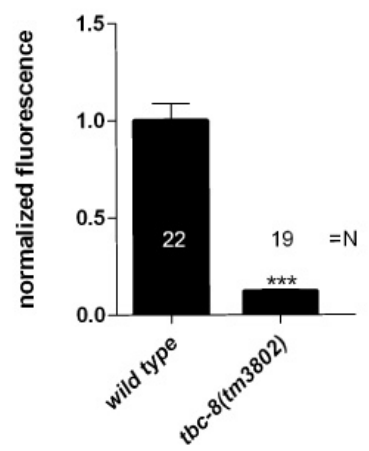

B

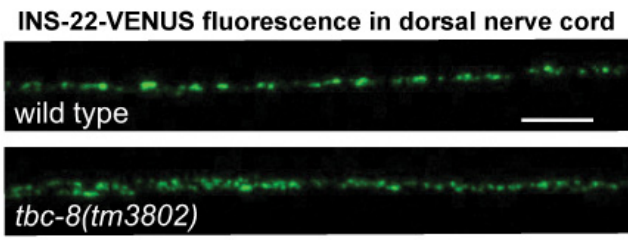

INS-22-VENUS in dorsal nerve cord

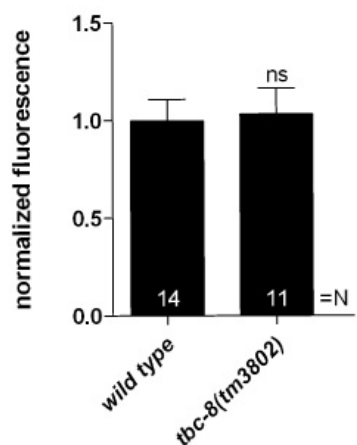

Figure IV.6. The VENUS-tag derived from other neuropeptides than NLP-21 was also lost in $\boldsymbol{t b c - 8 ( t m 3 8 0 2 )}$ mutants (A) The FMRFamide related peptide FLP-3-derived VENUS fluorescence was decreased in $t b c-8(t m 3802)$. (B) Fluorescence intensity of VENUS-tagged insulin-like neuropeptide, INS-22, was unchanged in $t b c-8(t m 3802)$ mutants. Scale bars in DNC represent $5 \mu \mathrm{m}$. Error bars $=$ s.e.m. $\left({ }^{* * *}, \mathrm{P}<0.0001\right.$; Student's t-test). Source: (Hannemann et al, 2012).

In addition to different neuropeptides, we also tested a transmembrane protein localized on DCVs, IDA-1-GFP, in the DNC. IDA-1-GFP levels were unchanged in the $t b c-8$ deletion mutants when compared to wild type animals, suggesting no changes in the numbers of DCVs in $t b c-8$ mutants (Figure IV.7). 
IDA-1-GFP fluorescence in dorsal nerve cord

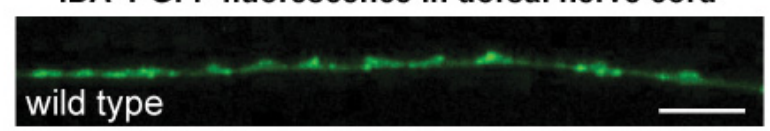

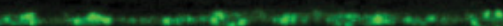

tbc-8(tm3802)
IDA-1-GFP

in dorsal nerve cord

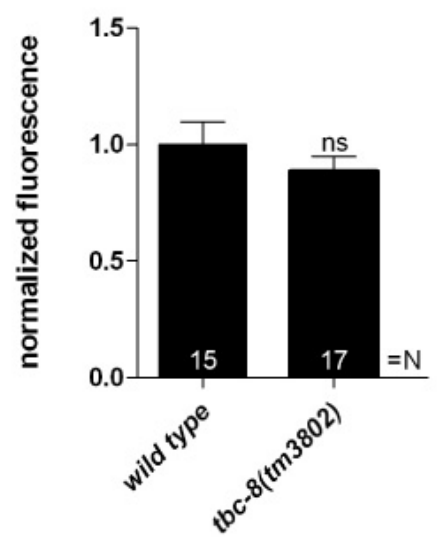

Figure IV.7. IDA-1-GFP trafficking is not affected in $t \boldsymbol{b c - 8}(\mathbf{t m} 3802)$ mutants. The fluorescence level of the transmembrane protein, IDA-1-VENUS, was unaltered in $t b c$ 8(tm3802) deletion mutant worms. Scale bars in DNC represent $5 \mu \mathrm{m}$. Error bars = s.e.m. (ns, $\mathrm{P}>0.05$, Student's t-test). Source: (Hannemann et al, 2012).

This observation was verified by a high-pressure freeze electron microscopy (HPF-EM). This analysis revealed that the DCV numbers and distribution at synapses in $t b c-8$ mutants were unaltered compared to wild type (Figure IV.15B). Therefore, we conclude that in $t b c-8$ mutants, DCVs are loaded with less soluble NLP-21-derived VENUS, as it has also been shown for unc-108/rab-2 mutants (Edwards et al, 2009; Sumakovic et al, 2009). Thus, DCV maturation is disrupted in tbc-8 mutants in a manner similar to unc-108/rab-2 mutants.

\section{IV.2 TBC-8 and RAB-2 are involved in the same genetic pathway}

In order to analyze whether TBC-8 acts in the same genetic pathway as RAB2, we compared $t b c-8(t m 3802)$ with different $u n c-108 / r a b-2$ mutants. We utilized two different alleles of RAB-2, (n501) D122N, which is dominant active and constitutively bound to GTP (Sumakovic et al, 2009), and the deletion (nu415) that serves as a molecular null allele since the protein product could not be detected on Western blots (Chun et al, 2008). In unc-108/rab-2(n501) NLP-21-derived VENUS fluorescence levels in the DNC were more strongly decreased as compared to the 
deletion allele (nu415) by $91.13 \pm 1.38 \%$ and $43.89 \pm 5.59 \%$, respectively (Figure IV.8). If TBC-8 is the GAP for RAB-2, then in $t b c-8$ mutants, RAB-2 should predominantly be in the constitutively GTP-bound form and therefore, $t b c-8$ mutants should resemble the dominant unc-108/rab-2(n501) mutant. Consistent with this hypothesis, the tbc8(tm3802) deletion allele indeed showed a decrease in NLP-21-derived VENUS fluorescence $(74.00 \pm 2.51 \%)$, similar to unc-108(n501) mutants $(91.13 \pm 1.38 \%)$ (Figure IV.8). Comparatively, the decrease in NLP-21-derived VENUS fluorescence was not as pronounced in the unc-108(nu415) null allele (43.89 $\pm 5.59 \%)$ (Figure IV .8). This result suggests that RAB-2 is indeed in the active GTP-bound form in $t b c$ 8 mutants. Therefore, inactivation of RAB-2 in a $t b c-8$ mutant background should lead to a weaker NLP-21-VENUS phenotype, identical to the unc-108/rab-2(nu415) null allele. In agreement with this hypothesis, unc-108(nu415); tbc-8(tm3802), a combination of both deletion alleles, showed an identical phenotype (46.88 $\pm 5.20 \%)$ as

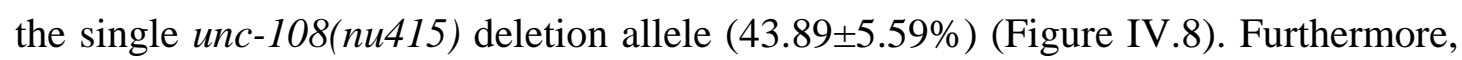
unc-108(n501); tbc-8(tm3802), a double mutant with unc-108/rab-2 gain of function allele, (91.84 $\pm 1.37 \%)$ also resembled the single unc-108(n501) allele (91.13 $\pm 1.38 \%)$ (Figure IV.8). Both double mutants followed the phenotype of the respective unc-108 single mutant indicating that both proteins are involved in the same genetic pathway.
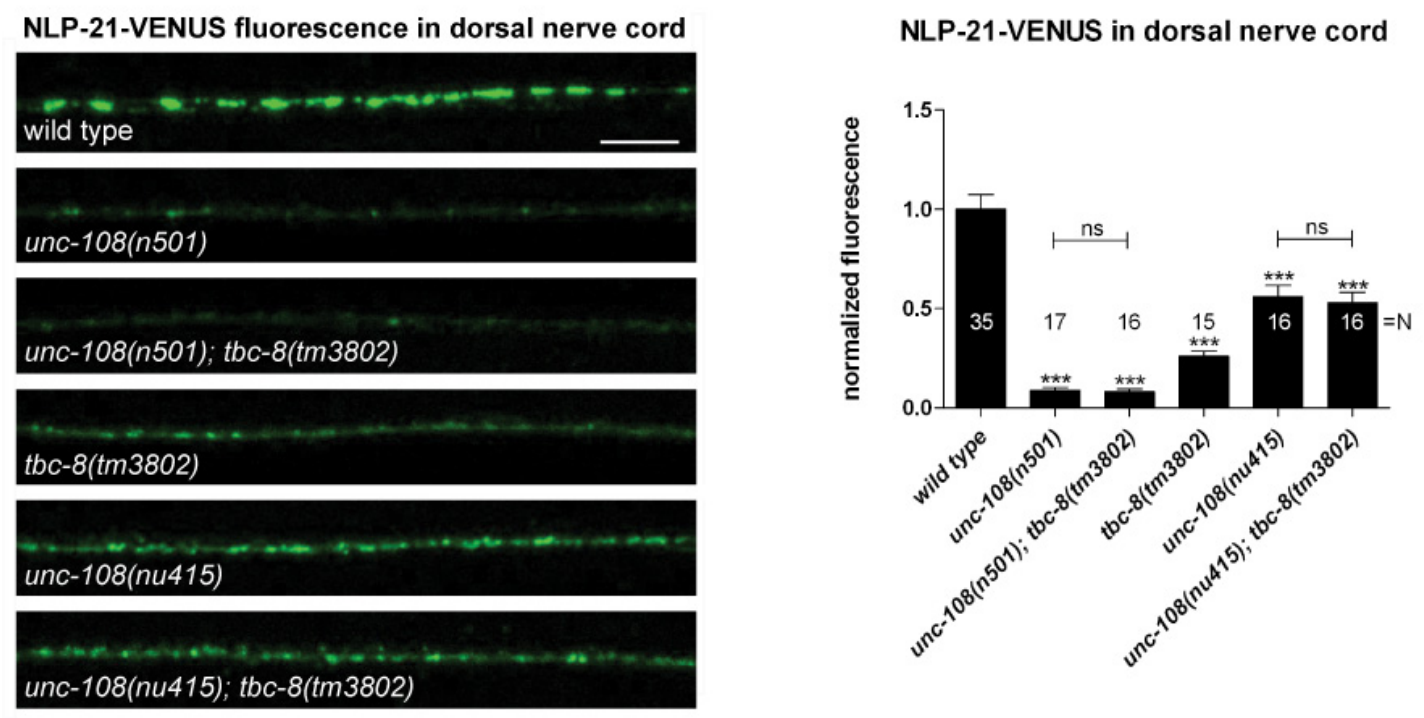

Figure IV.8. TBC-8 is involved in the same pathway as UNC-108/RAB-2. Double mutants of $t b c-8(t m 3802)$ and dominant active unc-108/rab-2(n501) or null allele unc-108/rab2(nu415), respectively, followed the phenotype of the unc-108/rab-2 single mutants. Scale bar represents $5 \mu \mathrm{m}$. Error bars $=$ s.e.m. ${ }^{* * *}, \mathrm{P}<0.0001$; Student's t-test). Source: (Hannemann et al, 2012). 
Previously, we and others have shown that cargo was lost in the endosomallysosomal pathway in unc-108/rab-2 mutants (Edwards et al, 2009; Sumakovic et al, 2009). In order to block this loss of DCV cargo to early endosomes, we overexpressed a constitutively GTP-bound form of RAB-5 (Q79L) in neurons and could rescue the DCV phenotype in the unc-108(n501) mutants by returning NLP-21-derived VENUS fluorescence levels back to wild type levels in the DNC (Sumakovic et al, 2009). Since TBC-8 and RAB-2 function in the same pathway, expression of RAB-5 (Q78L) should also rescue the decreased VENUS levels in the DNC in the tbc-8(tm3802) deletion allele. Consistent with this hypothesis, expression of RAB-5 (Q78L) in the neurons of $t b c-8(t m 3802)$ mutants restored the decreased NLP-21-derived VENUS fluorescence levels at the synapses up to $86.47 \pm 10.90 \%$ (Figure IV.9). This result indicates that TBC-8, like RAB-2, is required for retaining specific cargo in maturing DCVs by preventing its entry to the late endosomal system.
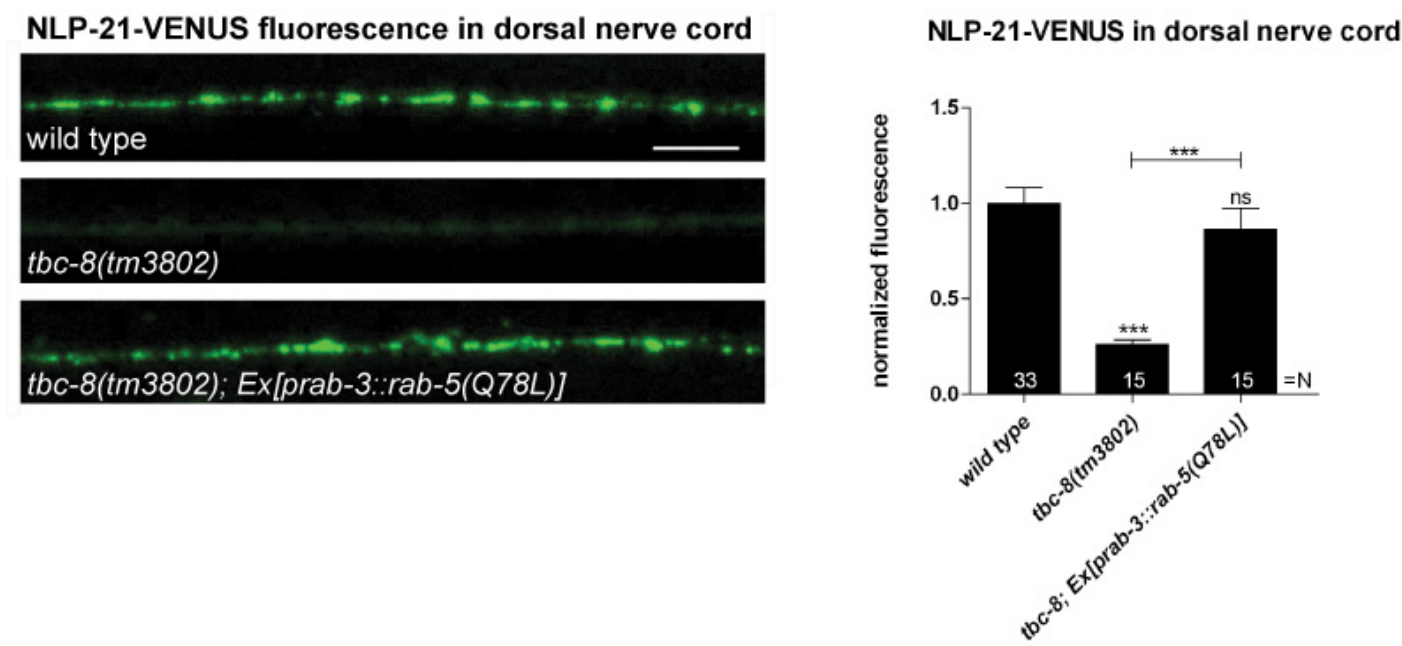

Figure IV.9. Loss of the soluble VENUS cargo in $t b c-8(t m 3802)$ mutants is rescued by overexpression of constitutively active, GTP-bound RAB-5 (Q78L), like in unc-108/rab-2 mutants (Sumakovic et al, 2009). Interfering with the endosomal-lysosomal pathway by overexpression of RAB-5 (Q78L) in a $t b c-8(t m 3802)$ mutant background restored the NLP21-derived VENUS fluorescence at the DNC back to wild type level. Scale bar represents 5 $\mu \mathrm{m}$. Error bars $=$ s.e.m. $\left({ }^{* * *}, \mathrm{P}<0.0001\right.$; Student's t-test). Source: (Hannemann et al, 2012). 


\section{IV.3 TBC-8 is not involved in neuropeptide processing}

Neuropeptides are synthesized as larger inactive proneuropeptides, which are processed by the action of different enzymes such as proprotein convertases EGL3/PC2. It was previously shown that EGL-3/PC2 is the major convertase in the biogenesis of active neuropeptides (Husson et al, 2006; Kass et al, 2001). In egl-3 mutant worms no active FMRFamide neuropeptides and neuropeptide-like proteins (NLP) could be detected by mass spectrometry (Husson et al, 2006). Due to absence of the proprotein convertase EGL-3, egl-3 mutants display defects in neuropeptide signaling like movement defects (Sumakovic et al, 2009) and defects in mechanosensory responses (Kass et al, 2001) like egg-laying behavior (Trent et al, 1983).

In order to test whether TBC-8 is involved in neuropeptide processing, we generated a double mutant of the prohormone convertase PC2, egl-3(gk238), and tbc8(tm3802), and tested it for defects in DCV maturation (Figure IV.10). The data was compared with the respective single mutants to identify possible genetic interactions. If TBC-8 and EGL-3 function in the same pathway, one would expect that double mutants of $t b c-8$ and egl-3 display the same phenotype like egl-3 single mutants. However, in egl-3(gk238); tbc-8(tm3802) double mutants, NLP-21-derived VENUS levels were restored to wild type levels (72.50土9.90\%) (Figure IV.10). Previously, it has been shown that the loss of NLP-21-derived VENUS from maturing DCVs in unc-108/rab-2 mutants could also be rescued by inactivation of EGL-3 (Sumakovic et al, 2009). Thus, the lack of proneuropeptide processing retains NLP-21-VENUS into the insoluble dense core of DCVs of egl-3(gk238); unc-108/rab-2 and egl-3(gk238); $t b c-8(t m 3802)$ double mutants. These findings indicate that primarily soluble NLP-21derived VENUS is lost in unc-108/rab-2 and tbc-8(tm3802) mutants. 
NLP-21-VENUS fluorescence in dorsal nerve cord
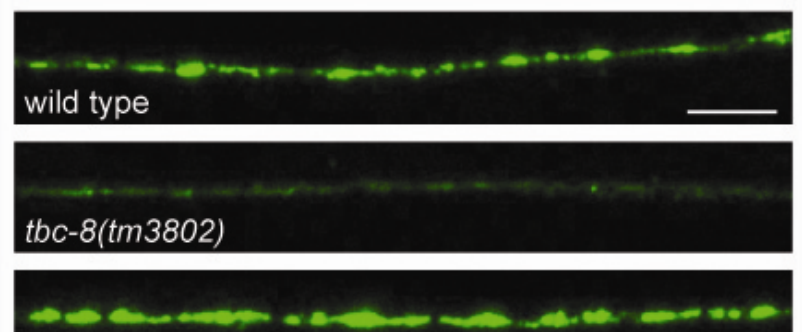
egl-3(gk238)

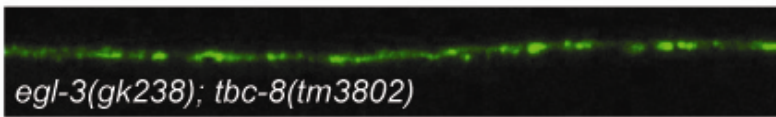

NLP-21-VENUS in dorsal nerve cord

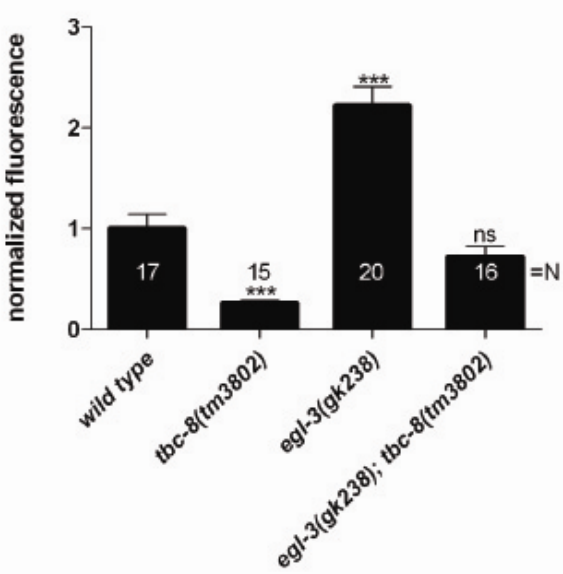

Figure IV.10. TBC-8 is not involved in the generation of active NLP-21 neuropeptides. The double mutant egl-3(gk238); tbc-8(tm3802) restored NLP-21-derived VENUS cargo in the DNC back to wild type levels. Scale bar represents $5 \mu \mathrm{m}$. Error bars $=$ s.e.m. $\left({ }^{* * *}, \mathrm{P}<\right.$ 0.0001; Student's t-test). Source: (Hannemann et al, 2012).

Although unc-108/rab-2; egl-3(gk238) double mutants had restored levels of NLP-21-derived VENUS in the DNC, analysis of the movement phenotype in the double mutants revealed that they were more severely uncoordinated than either single mutant (Sumakovic et al, 2009). This result suggests that, despite rescuing the NLP-21-derived VENUS marker in the DNC, the combined loss of soluble cargo and functional neuropeptides in DCVs leads to additive locomotion defects in unc108/rab-2; egl-3(gk238) double mutants. Interestingly, tbc-8(tm3802) mutants do not display a movement defect (Figure IV.11) and double mutants of tbc-8(tm3802); egl3(gk238) do not yield animals that are similarly uncoordinated to unc-108/rab-2; egl3(gk238) mutants (Figure IV.12) (Sumakovic et al, 2009). These data suggest that role of RAB-2 in regulating movement may predominantly occur in a subset of neurons that do not express $t b c-8$, but most likely express another GAP. 


\section{Movement Assay}

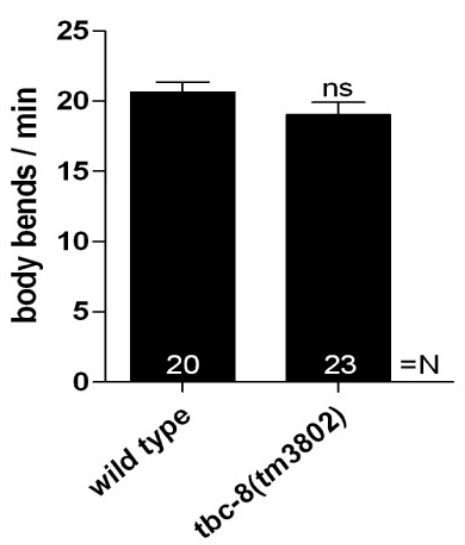

Figure IV.11. $t b c-8(t m 3802)$ mutants do not display movement defects. Young adult worms of each strain were transferred to non-seeded plates, adjusted for several minutes before the number of body bends per min of each worm was recorded. tbc- 8 mutants displayed normal rate of locomotion when compared to wild type animals. Error bars $=$ s.e.m. (ns, P > 0.05; Student's t-test). Source: (Hannemann et al, 2012).

\section{Movement Assay}

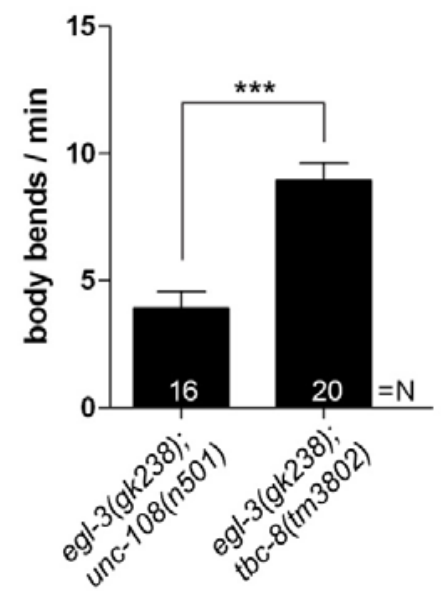

Figure IV.12. Unlike egl-3(gk238); unc-108/rab-2(n501) mutants, double mutants of egl$3(g k 238)$ and $t b c-8(t m 3802)$ display no additive movement defects. It was previously shown that the combined loss of soluble cargo and functional neuropeptides in DCVs leads to additive locomotion defects in unc-108/rab-2(n501); egl-3(gk238) double mutants (Sumakovic et al, 2009). However, double mutants of tbc-8(tm3802); egl-3(gk238) do not show similarly uncoordinated movement to unc-108/rab-2(n501); egl-3(gk238) mutants. Error bars $=$ s.e.m. $\left({ }^{* * *}, \mathrm{P}<0.0001\right.$; Student's t-test $)$.

All these findings highlight that TBC-8 is indeed in the same pathway as RAB-2 and thus, TBC-8 is a likely candidate to be a RAB-2 GAP. However, differences in movement defects between $t b c-8$ and unc-108/rab-2 mutants suggest 
the existence of additional GAPs, which may regulate RAB-2 activity in the absence of TBC-8.

\section{IV.4 tbc-8 mutants show no morphological defects in motoneurons and no SV defects}

For unc-108/rab-2 mutants, it has been shown that DCV maturation is specifically affected while SV trafficking and function are unaltered (Edwards et al, 2009; Sumakovic et al, 2009). To exclude that the lack of TBC-8 would lead to general membrane trafficking defects in neurons, we analyzed SV localization, distribution and morphology. We first examined synaptic morphology by analyzing the localization of the synaptic marker proteins, RAB-3 and Synaptobrevin-1 (SNB1), using integrated strains expressing YFP-RAB-3 and GFP-SNB-1 in cholinergic motoneurons (Sieburth et al, 2005). The fluorescence of YFP-RAB-3 (90.12 $\pm 11.96 \%)$ (Figure IV.13A) and GFP-SNB-1 (90.82 $\pm 9.32 \%$ ) (Figure IV.13B) was unchanged in the DNC of $t b c-8(t m 3802)$ when compared to wild type.
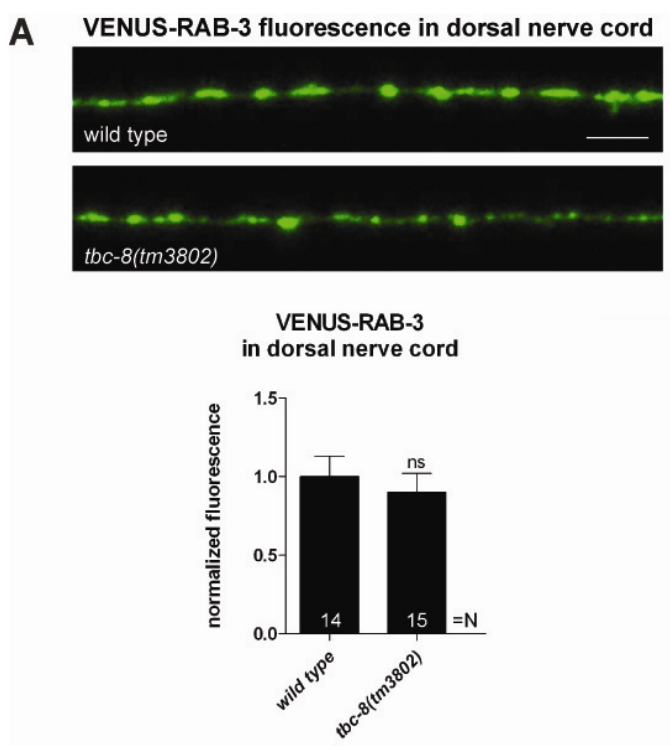

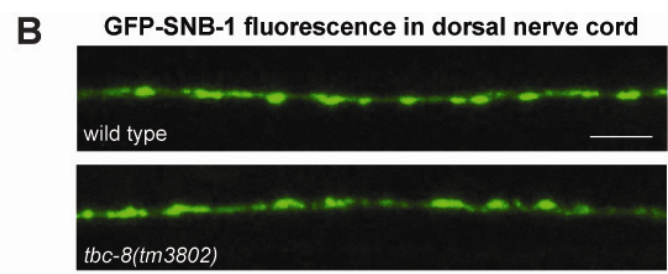

GFP-SNB-1 in dorsal nerve cord

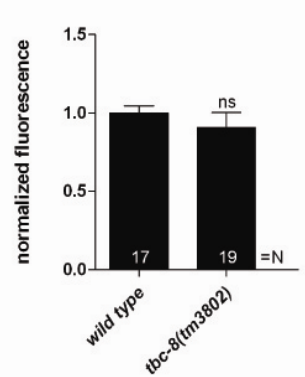

Figure IV.13. $t b c-8(t m 3802)$ mutants display no defects in synaptic vesicle trafficking. The transport of synaptic vesicles to the synapses in the DNC was visualized by VENUStagged RAB-3 (VENUS-RAB-3) (A) and GFP-tagged synaptobrevin (GFP-SNB-1) (B). No differences were observed in transport of both SV markers in tbc-8(tm3802) mutants. Scale bar represents $5 \mu \mathrm{m}$. Error bars $=$ s.e.m. (ns, P > 0.05; Student's t-test). Source: (Hannemann et al, 2012). 
Second, we used HPF-EM to detect obvious changes in the morphology of synapses, neuronal cell bodies and neuronal Golgi complexes in tbc-8(tm3802) mutants compared to wild type worms (Figure IV.14). Here, we did not observe any abnormal accumulation of vesicles or degeneration of the Golgi stacks in tbc8(tm3802) mutants compared to wild type animals.

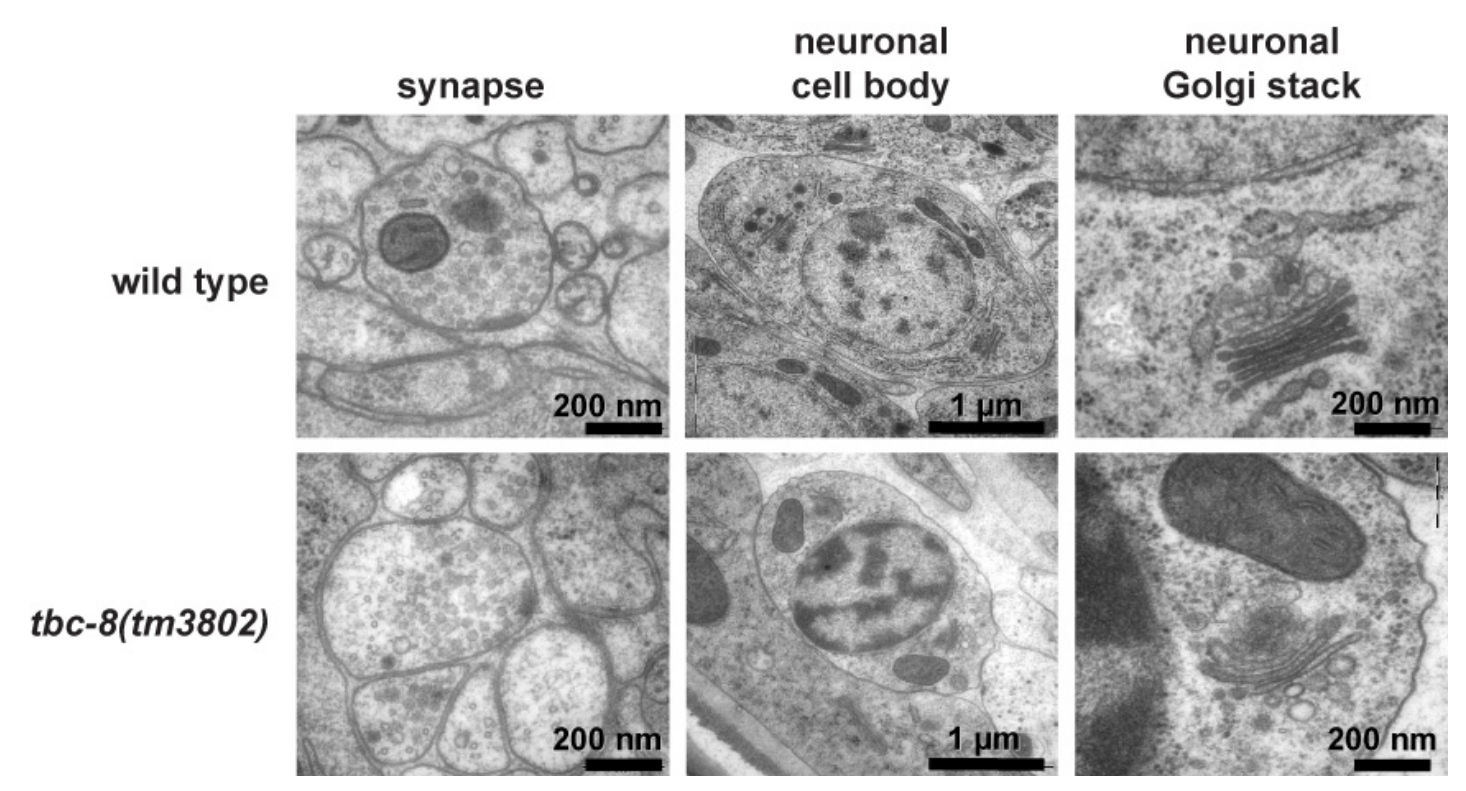

Figure IV.14. $t b c-8(t m 3802)$ mutants do not show morphological changes. High-pressure freeze electron microscopic analysis of $t b c-8(t m 3802)$ mutants revealed no obvious differences in the morphology of synapses, motoneuronal cell bodies and neuronal Golgi stacks compared to wild type. EM pictures were taken by Dr. Jan Hegermann. Source: (Hannemann et al, 2012).

Furthermore, we analyzed SV distributions surrounding the presynaptic dense projection of cholinergic motoneurons in $t b c-8(t m 3802)$ mutants by HPF-EM. There were no changes in SV distribution, SV numbers or SV diameter as compared to wild type (Figure IV.15). This analysis suggests that SV function is unaffected in $t b c$ 8(tm3802) mutants, similar to what has been reported for unc-108/rab-2 mutants (Edwards et al, 2009; Sumakovic et al, 2009). For unc-108/rab-2 gain of function mutants, it has been demonstrated that DCV diameters are more variable (Edwards et al, 2009; Sumakovic et al, 2009) (Figure IV.15B). However, this was not the case for tbc-8(tm3802) mutants, where DCV diameters are not significantly different from DCVs in wild type worms (Figure IV.15B). 
A synaptic vesicle distribution

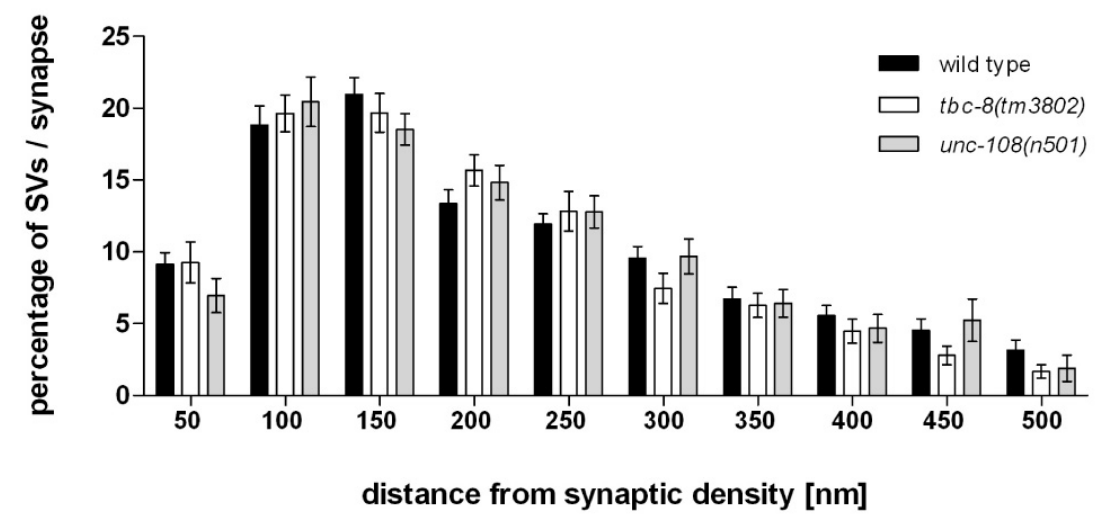

B

\begin{tabular}{lcccccc}
\hline Genotype & $\begin{array}{c}\text { Number of } \\
\text { profiles } I \\
\text { animals } \\
\text { analyzed }\end{array}$ & $\begin{array}{c}\text { Mean area } \\
\text { of presynaptic } \\
\text { terminal in } \\
\text { cross sections } \\
{\left[\mu^{2}\right]}\end{array}$ & $\begin{array}{c}\text { SV I } \\
\text { profile }\end{array}$ & $\begin{array}{c}\text { Mean diameter } \\
\text { of SV [nm] } \\
\text { (number of } \\
\text { analyzed SV) }\end{array}$ & $\begin{array}{c}\text { DCV I } \\
\text { profile }\end{array}$ & $\begin{array}{c}\text { Mean diameter } \\
\text { of DCV [nm] } \\
\text { (number of } \\
\text { analyzed DCV) }\end{array}$ \\
\hline wild type & $79 / 8$ & $0.21 \pm 0.01$ & $33.7 \pm 1.2$ & $29.6 \pm 0.7(89)$ & $2.1 \pm 0.2$ & $43.6 \pm 0.8(49)$ \\
tbc-8(tm2302) & $48 / 3$ & $0.19 \pm 0.01$ & $37.3 \pm 1.5$ & $30.0 \pm 0.4(41)$ & $2.0 \pm 0.4$ & $41.5 \pm 0.7(77) \mathrm{ns}$ \\
unc-108(n501) & $25 / 3$ & $0.24 \pm 0.04$ & $38.2 \pm 1.9$ & $27.8 \pm 0.7(97)$ & $2.3 \pm 0.3$ & $49.2 \pm 1.6(59){ }^{* *}$ \\
\hline
\end{tabular}

Figure IV.15. $t b c-8(t m 3802)$ mutants display no defects in synaptic vesicle trafficking and localization. (A) The synaptic vesicle distribution relative to the presynaptic density at synapses of $t b c-8(t m 3802)$ mutants was similar to wild type worms (Student's t-test). (B) SV and DCV analysis determined by HPF EM. Only significant differences of mutant strains compared to wild type worms are indicated. Mean \pm s.e.m. are shown $(* *, \mathrm{P}<0.001$; Student's t-test). The mean diameter of DCVs in unc-108(n501) mutants was more viable, which was shown previously (Sumakovic et al, 2009). EM was performed by Dr. Jan Hegermann. Source: (Hannemann et al, 2012).

IV.5 tbc-8 codes for an evolutionarily conserved Rab GAP specifically expressed in neurons

The $t b c-8$ gene $(\mathrm{C} 38 \mathrm{H} 2.1)$ of $C$. elegans is predicted to consist of 18 exons that are composed of 2712 base pairs (bp) (Figure IV.16). Based on the published genome sequence of $C$. elegans, $t b c-8$ is located on the third chromosome on the plus strand between the nucleotides 10,365,002 and 10,373,138 (Wilson et al, 1994) (www.wormbase.org). In this study the complete coding region of $t b c-8$ could be confirmed by sequencing of the $t b c-8$ cDNA. The immediate neighbors of $t b c-8$ are umps-1 (T07C4.1) and the gene C38H2.2, which encode an uridine-5'-monophosphate 
synthase and a core 1 UDP-Gal:GalNAc alpha1-Ser/Thr beta1,3-galactosyltransferase (EC2.4.1.122), respectively (www.wormbase.org).

The $t b c-8(t m 3802)$ deletion allele, which was used in this study leads to a stop codon after the $8^{\text {th }}$ exon (Figure IV.16), truncating the protein after aa 482 just before the TBC-domain starts (Figure IV.17).

\section{tbc-8}

length: $8137 \mathrm{bp}$

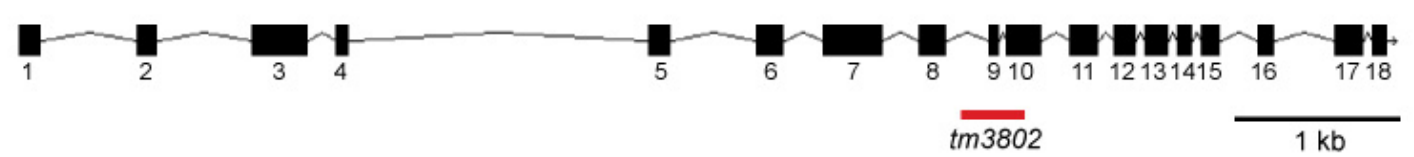

Figure IV.16. Gene structure of $\boldsymbol{t b c - 8}$. Exons are represented by black boxes and introns by black lines. The position of the $t m 3802$ deletion is indicated by a red line. The genomic region of the $t b c-8$ locus consists of $8137 \mathrm{bp}$, whereas the cDNA of $t b c-8$ consists of $2712 \mathrm{bp}$. This figure was created by the software "X-on”, developed by Dr. Christian Olendrowitz. Source: (Hannemann et al, 2012).

The gene $t b c-8$ encodes a 903 amino acid (aa) protein. This protein product has a predicted size of $104 \mathrm{kDa}$ and a theoretical pI of $6.5 \mathrm{TBC}-8$ is predicted to be a cytoplasmic protein as the TMHMM program did not find any transmembrane domains (http://www.cbs.dtu.dk/services/TMHMM/). TBC-8 possesses two predicted protein domains, a TBC domain and a RUN domain (http://smart.emblheidelberg.de/). The TBC-domain of TBC-8 is located at the C-terminus between aa 621 and 862 (Figure IV.17). The conserved RUN [after RPIP8 (RaP2 interacting protein 8)/UNC-14/NESCA(new molecule containing SH3 at the carboxyl-terminus)] domain is located at the N-terminus between aa 96 and 228 (Figure IV.17), which has been shown to bind to small GTPases of the Rab and Rap family (Callebaut et al, 2001; Janoueix-Lerosey et al, 1998). TBC-domain proteins with the same domain structure can be found in Drosophila melanogaster and the mammalian system (Fukuda, 2011) (Figure IV.17). The orthologs in D. melanogaster (CG32506-PC, FlyBase ID: FBpp0300194) and in H. sapiens (SGSM1 (Yang et al, 2007), accession number: NP_001035037) are about 250 aa longer than C. elegans TBC-8. The RUN domains of these proteins show a 64\% (SGSM1) and a 63\% (CG32506-PC) similarity to the RUN domain of TBC-8, whereas both TBC-domains are about 58\% (SGSM1) 
and 57\% (CG32506-PC) similar to the TBC-domain of C. elegans TBC-8. For full protein sequences of TBC-8 and its orthologs see Appendix (Figure XV.1).

C. elegans
TBC-8

H. sapiens SGSM1

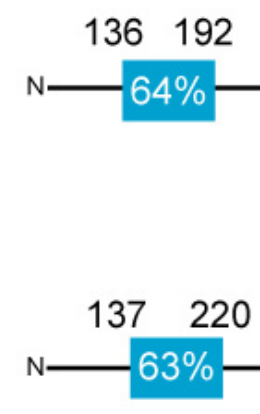

D. melanogaster CG32506-PC
890

$58 \%-c$
1148 aa

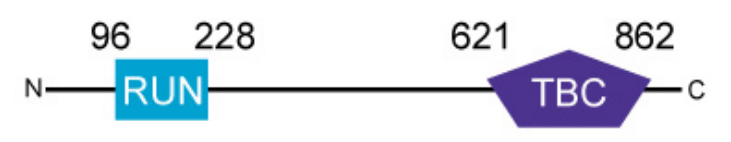

903 aa

1148 aa

In order to determine the expression pattern of $t b c-8$, a construct containing a 2873 bp genomic fragment of the tbc-8 promoter region was fused to $\mathrm{gfp}$ and injected into wild type worms (Figure IV.18A). The expression pattern using this transcriptional reporter revealed that $t b c-8$ is exclusively expressed in neurons including the head and tail neurons as well as neurons in the ventral nerve cord (VNC) (Figure IV.18B). Interestingly, analysis of the expression pattern of unc-108/rab-2 showed a similar high expression in neurons (Edwards et al, 2009; Sumakovic et al, 2009). 
A

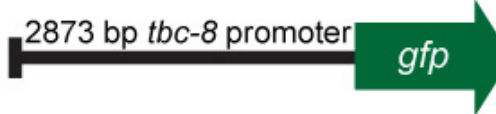

B
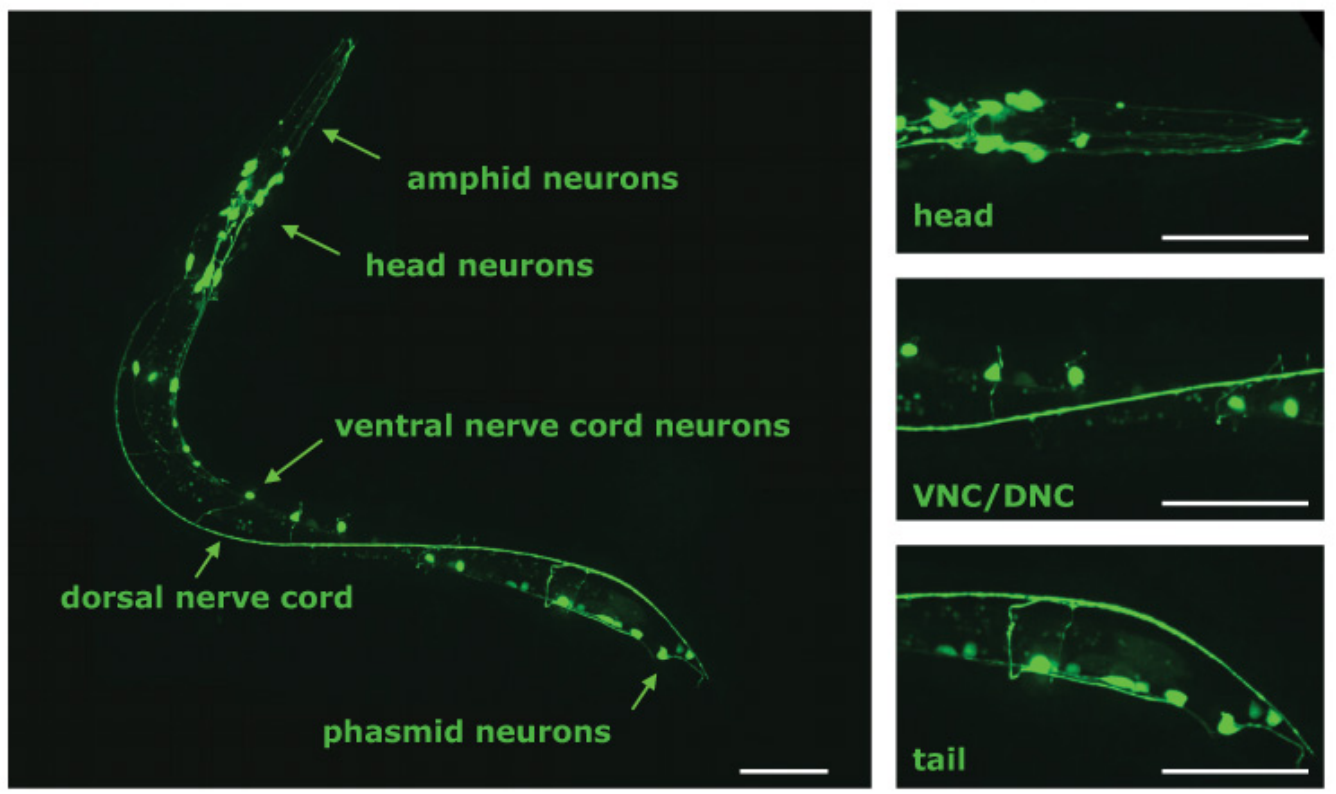

Figure IV.18. $t b c-8$ is expressed in the nervous system of $C$. elegans. (A) Schematic representation of a construct containing a 2873 bp tbc-8 promoter fused to $g f p$. This transcriptional reporter construct was injected into wild type worms and $g f p$ expression was imaged in stage three larval (L3) worms by confocal microscopy. (B) $t b c-8$ expression was observed in the neurons of the head including amphid neurons, the ventral nerve cord (VNC) neurons and in neurons of the tail (phasmid neurons). The right panel shows magnifications of the respective regions. Scale bars represent $50 \mu \mathrm{m}$. DNC, dorsal nerve cord. Source: (Hannemann et al, 2012).

IV.6 TBC-8 shows no defects in postendocytic trafficking and is not required for degradation of apoptotic cell corpses

Based on our expression analysis, we could show that $t b c-8$ is expressed in the nervous system (Figure IV.18). To exclude the possibility that our transcriptional reporter missed important regulatory regions and elements, we tested TBC-8 function in other tissues in which RAB-2 was shown to be required (Chun et al, 2008; Lu et al, 2008; Mangahas et al, 2008). We analyzed the possible role of TBC-8 in postendocytic trafficking in coelomocytes (Figure IV.19-21) and in the degradation of apoptotic cell corpses in the germ line (Figure IV.22). 
In order to observe defects in the steady-state endocytosis of coelomocytes, we used the integrated strain arIs37[pmyo-3::ssGFP], which continuously secretes ssGFP from muscle cells into the body cavity of $C$. elegans (Fares \& Greenwald, 2001). Subsequently, ssGFP is endocytosed by the macrophage-like coelomocytes, which continuously filter the body fluid. By imaging the endocytosed ssGFP fluorescence in coelomocytes, we did not detect any changes during the steady-state endocytosis of ssGFP in $t b c-8(t m 3802)$ mutants compared to wild type coelomocytes (Figure IV.19).

A

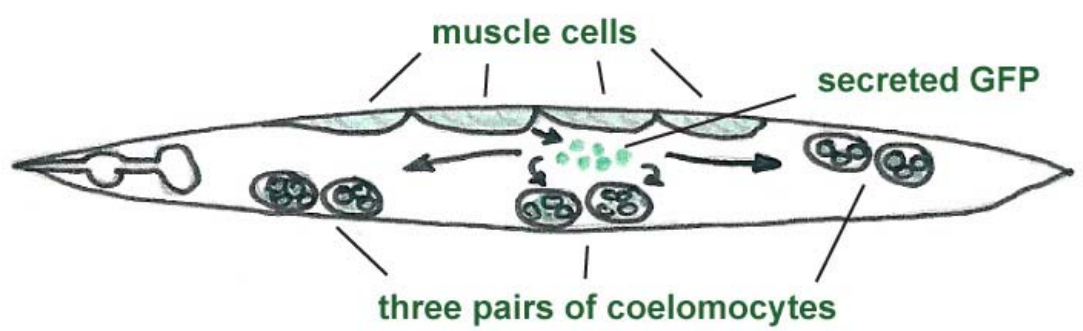

B
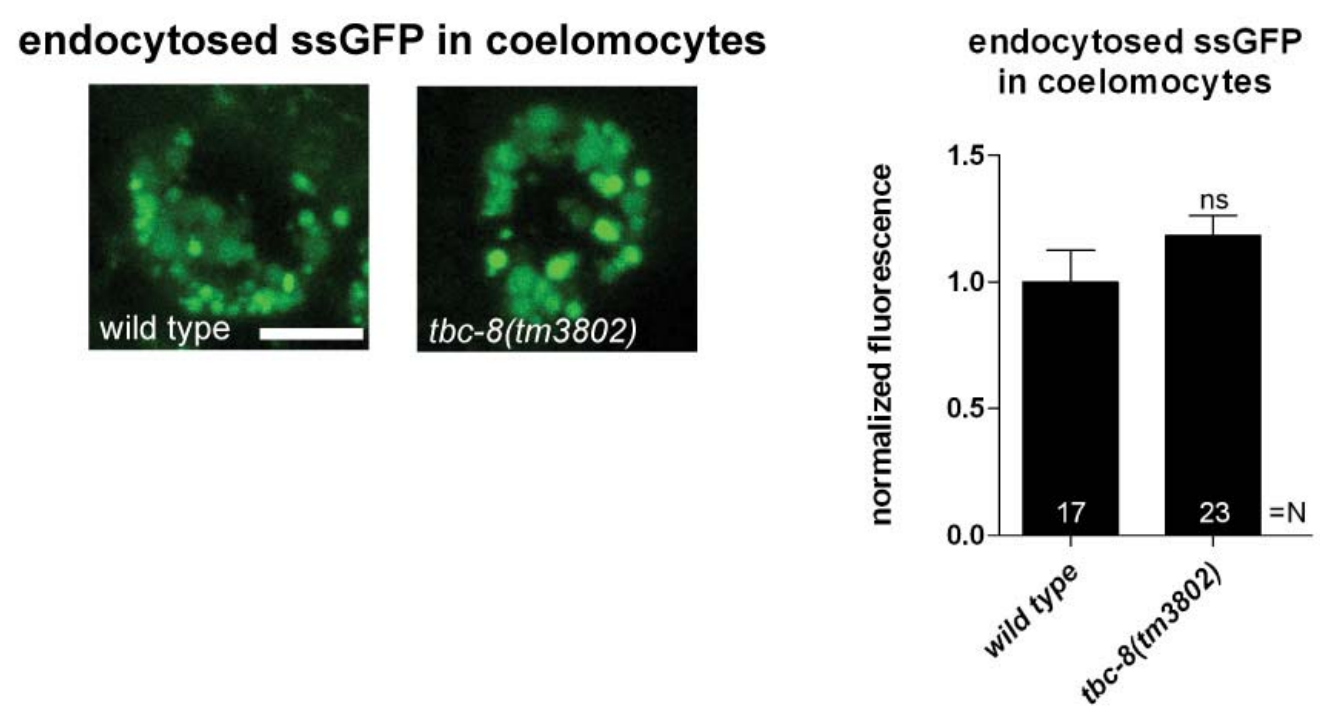

Figure IV.19. Steady-state endocytosis of coelomocytes in $t b c-8$ mutants is unaffected. (A) Schematic representation of the C. elegans strain arIs37[pmyo-3::ssGFP] (Fares \& Greenwald, 2001). This strain constitutively secretes ssGFP from muscle cells, which is endocytosed by the six coelomocytes of C. elegans. (B) $t b c-8(t m 3802)$ mutants were crossed into the strain arIs37[pmyo-3::ssGFP]. The fluorescence of endocytosed GFP in coelomocytes of $t b c-8$ mutants was imaged and compared to the fluorescence levels seen in a wild type background. Representative pictures of ssGFP in coelomocytes are shown. Scale bar represents $5 \mu \mathrm{m}$. Error bars $=$ s.e.m. (ns, P > 0.05, Student's t-test). Source: (Hannemann et al, 2012). 
In order to test the kinetics of ssGFP uptake and the degradation of endocytosed GFP in coelomocytes, we used a strain that expresses ssGFP under a heat-shock promoter (arIs36[phsp::ssGFP] (Fares \& Greenwald, 2001), which allowed us to perform an in-vivo pulse-chase experiment in C. elegans. In this strain, there is no $g f p$ expression at normal growth temperature of $20^{\circ} \mathrm{C}$. A heat shock at $33^{\circ} \mathrm{C}$ for $30 \mathrm{~min}$ results in the secretion of a finite pulse of GFP into the body cavity. Subsequently, the uptake and degradation of GFP can then be followed as a function of time by returning the heat-shocked worms back to $20^{\circ} \mathrm{C}$. While following the fate of secreted GFP within coelomocytes, no defects were observed in tbc-8(tm3802) mutants compared to wild type worms for all measured time points (Figure IV.20). This result indicates that, like the steady-state endocytosis, the rate of fluid-phase endocytosis in coelomocytes is also unaffected in $t b c-8$ mutant worms.

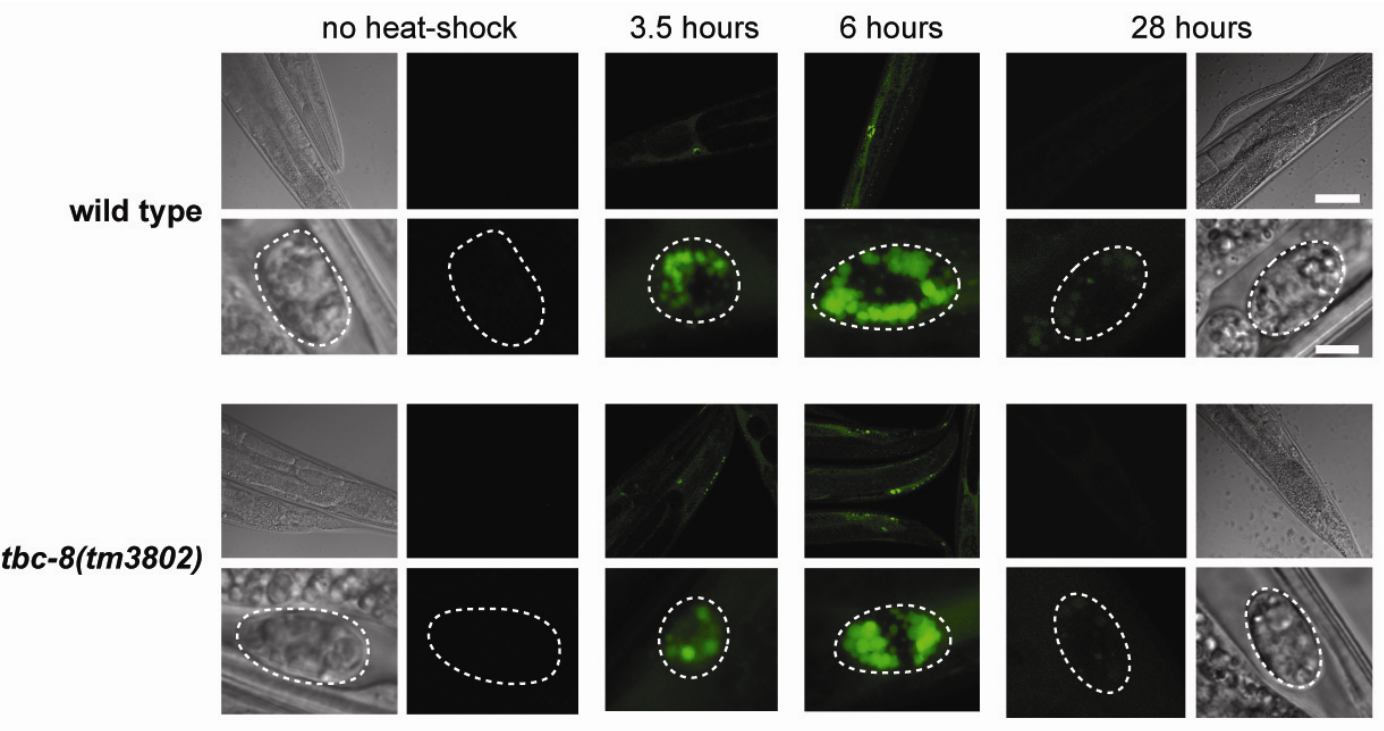

Figure IV.20. The fluid-phase endocytosis is unaffected in $t \boldsymbol{t b}-\boldsymbol{8}$ mutants. The strain arIs36[phsp::ssGFP] was crossed into tbc-8(tm3802). Both strains, arIs36 (wild type) (upper panel) and $t b c-8(t m 3802)$; arIs36 (lower panel) were grown at $20^{\circ} \mathrm{C}$, heat-shocked for $30 \mathrm{~min}$ at $30^{\circ} \mathrm{C}$ and returned at $20^{\circ} \mathrm{C}$ to allow recovery. After various time points (3.5 hours, 6 hours and 28 hours after heat-shock) both strains were monitored for uptake of ssGFP into coelomocytes and degradation of endocytosed GFP by confocal microscopy. All fluorescence pictures were taken with the same settings. Dashed lines indicate outlines of coelomocytes. Scale bar of worm sections represent $50 \mu \mathrm{m}$. Scale bar of coelomocytes represent $5 \mu \mathrm{m}$. Source: (Hannemann et al, 2012). 
In addition, we also examined postendocytic trafficking of the fluid-phase marker Texas red conjugated to the carrier protein bovine serum albumin (TR-BSA) in coelomocytes of $t b c-8(t m 3802)$ mutants. TR-BSA was microinjected into the pseudocoelom of young adult worms. Using a marker for endosomes, RME-8-GFP (Zhang et al, 2001), we analyzed the migration of injected TR-BSA through RME-8GFP positive vesicles into later postendocytic compartments (Figure IV.21). Within the first 5 to 10 min after injection, the dye TR-BSA is readily endocytosed by coelomocytes in wild type and $t b c-8$ mutant worms and accumulates in RME-8-GFP positive vesicles. After 30 min the marker leaves early endosomes and migrates into late endosomal compartments which are RME-8-GFP negative. This TR-BSA traffic was comparable in both wild type and tbc-8 mutants. Finally, after 50 min almost all TR-BSA accumulates in late compartments/lysosomes, in which Texas red is stable, and cannot be degraded (except for the BSA moiety). In summary, we did not see obvious defects at any time point in tbc-8(tm3802) mutants compared to wild type animals.

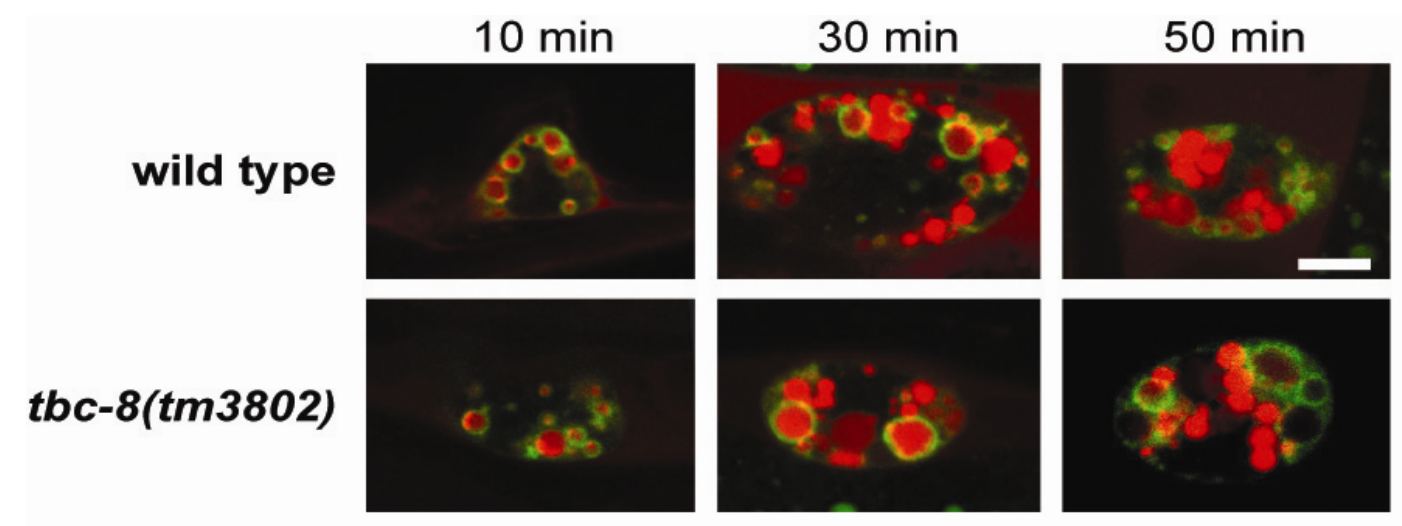

Figure IV.21. Fluid-phase endocytosis of Texas red-BSA was unaltered in $t b c-8(t m 3802)$ mutants. The endocytosis marker TR-BSA was injected into the body cavity of tbc$8(t m 3802)$ worms and its fate within coelomocytes was followed over time $(10 \mathrm{~min}, 30 \mathrm{~min}$, $50 \mathrm{~min}$ ). For this purpose, the strain bIs34[prme-8::rme-8-gfp], which labels RME-8 positive endosomes, was crossed into $t b c-8(t m 3802)$. After $10 \mathrm{~min}$, TR-BSA (red) was endocytosed and was visible in RME-8-GFP (green) positive vesicles in both $t b c-8$ (tm3802) mutants and in wild type worms. Therefore, endocytosis of the fluid-phase marker seemed to be unaffected in tbc-8(tm3802) mutants. Further observation of the kinetics in postendocytic trafficking of TRBSA (30 min, $50 \mathrm{~min}$ ) did not revealed any defects in tbc-8(tm3802) mutants. Scale bar represents $5 \mu \mathrm{m}$. Source: (Hannemann et al, 2012). 
Previously, it was shown that RAB-2 is also required for the degradation of apoptotic cell corpses in C. elegans (Lu et al, 2008; Mangahas et al, 2008). During this process, RAB-2 is crucial for the recruitment and fusion of lysosomes to phagosomes as well as the acidification of the phagosomal lumen ( $\mathrm{Lu}$ et al, 2008; Mangahas et al, 2008). To identify whether TBC-8 plays a role together with RAB-2 in the germ line, we assayed the presence of apoptotic cell corpses in $t b c-8(t m 3802)$ mutants (Figure IV.22). Unlike unc-108/rab-2 deletion mutants, tbc-8(tm3802) mutants showed similar numbers of apoptotic cell corpses (CED-1-GFP labeled) as wild type worms (Figure IV.22).
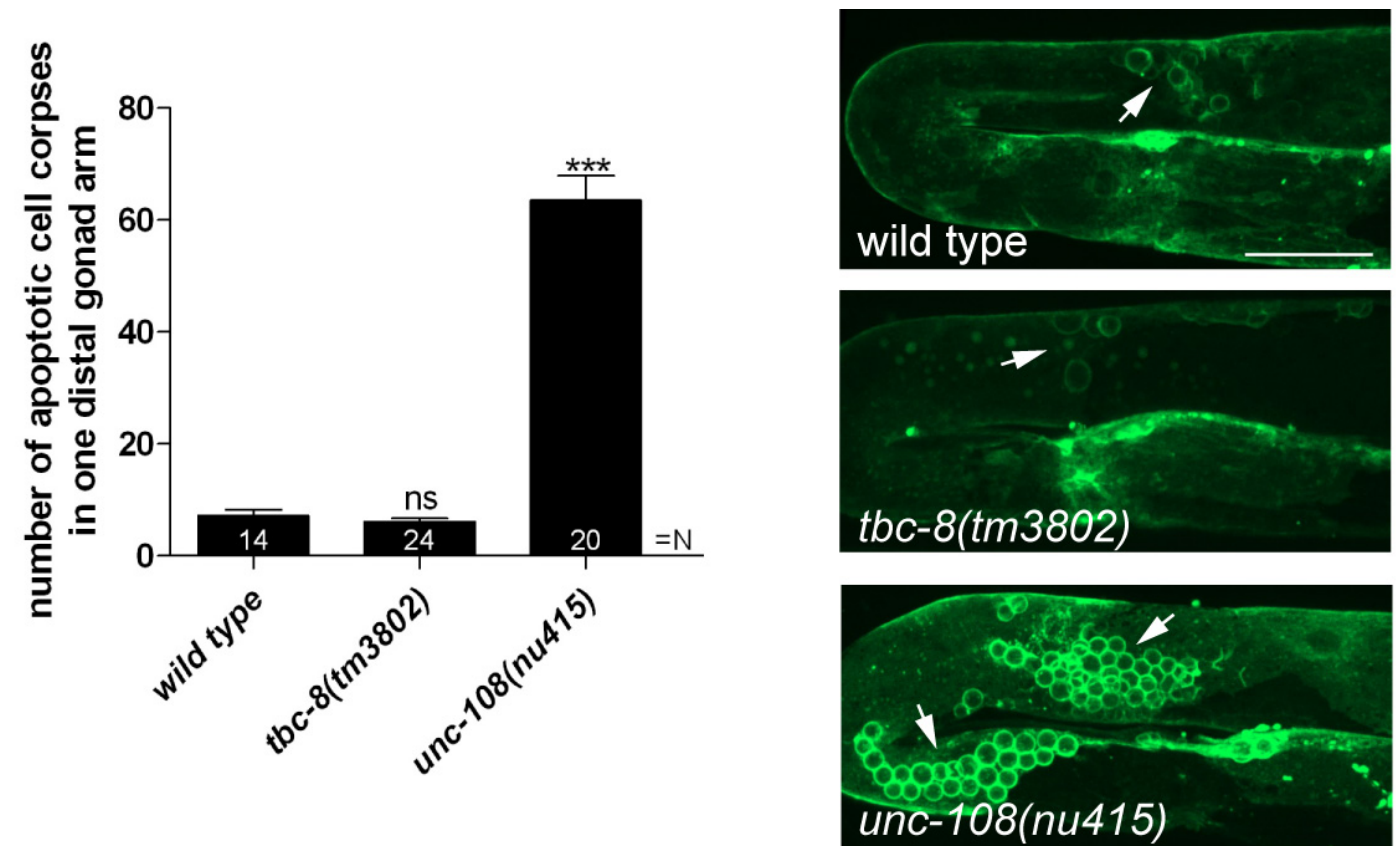

Figure IV.22. $t b c-8(t m 3802)$ mutants do not show defects in degradation of apoptotic cell corpses in the germ line. CED-1-GFP [bcIs39; (Schumacher et al, 2005)] was used as a marker to visualize apoptotic cell corpses in the germ line of $C$. elegans. Imaging stacks of one gonad arm were captured and the number of CED-1GFP positive apoptotic cell corpses was recorded. $t b c-8(t m 3802)$ mutants displayed similar numbers of apoptotic cell corpses like wild type worms, whereas unc108(nu415) mutants have defects in the degradation of apoptotic cell corpses resulting in high numbers of corpses in their gonad arms, which was described previously ( $\mathrm{Lu}$ et al, 2008; Mangahas et al, 2008). Scale bar represents $20 \mu \mathrm{m}$. Error bars = s.e.m. (***, P < 0.0001; Student's t-test). Source: (Hannemann et al, 2012). 
These postendocytosis data together with the expression analysis indicate that TBC-8 likely regulates RAB-2 function only in the nervous system of C. elegans. Therefore, it is tempting to speculate that RAB-2 is controlled by another GAP in non-neuronal tissues.

\section{IV.7 TBC-8 localizes to the Golgi-endosomal interface}

In order to examine where TBC-8 functions in the nervous system, we expressed fluorescently labeled TBC-8 under the $r a b-3$ pan-neuronal promoter and determined its sub-cellular localization in motoneurons of the VNC. Fluorescently labeled TBC-8 showed a cytosolic localization with some punctate membrane staining (Figure IV.23) reminiscent of RAB-2. In order to identify which subcellular compartments these cytoplasmic puncta correspond to, we co-localized fluorescentlylabeled TBC-8 with different markers in neurons (Figure IV.23). We observed partial localization of TBC-8 with medial (mannosidase II) and trans-Golgi (APT-9) markers and an almost complete co-localization with the early endosomal marker RAB-5. However, we could not observe any localization of TBC-8 to RAB-7-positive late endosomes. This suggests that TBC-8 acts at the Golgi-endosomal interface, similar to RAB-2, where DCV maturation is also believed to take place (Tooze et al, 2001). 

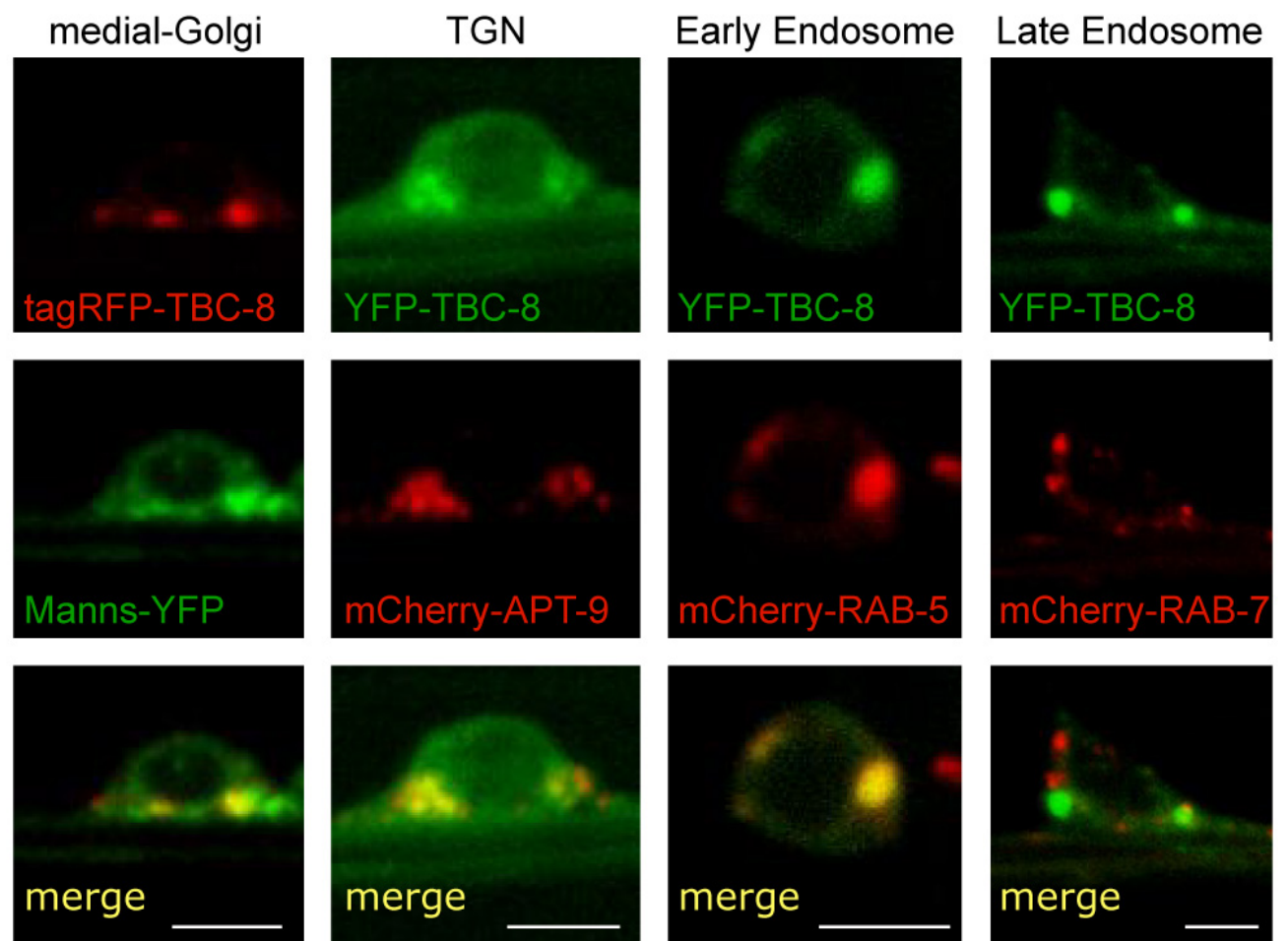

Figure IV.23. TBC-8 localizes to the Golgi-endosomal interface. The subcellular localization of fluorescently-labeled TBC-8 expressed in motoneurons was analyzed by confocal microscopy. Fluorescently-labeled-TBC-8 localized partially with medial and transGolgi markers and showed full co-localization with the early endosomal marker mCherryRAB-5. No co-localization to RAB-7-positive late endosomes was observed. Manns, mannosidase II. Scale bars represent $3 \mu \mathrm{m}$. Source: (Hannemann et al, 2012).

\section{IV.8 TBC-8 full length and fragments of TBC-8 are not soluble when expressed} in $E$. coli strains or insect cells.

The NLP-21 rescue experiments suggested that TBC-8 possess a catalytic GAP domain, represented by the TBC-domain, since a catalytic inactive TBC-8 (R697A) protein was not able to rescue the decreased NLP-21-derived fluorescence levels in $t b c-8$ deletion mutants. The proof-of principle experiment to determine GAP activity of TBC-8 towards RAB-2 would be an in-vitro GAP assay. Therefore, we attempted to express full-length TBC-8 or TBC-domain fragments of TBC-8 in E. coli. For this purpose, we cloned different constructs of TBC-8 (Figure IV.24) into expression vectors (Table IV.1). 


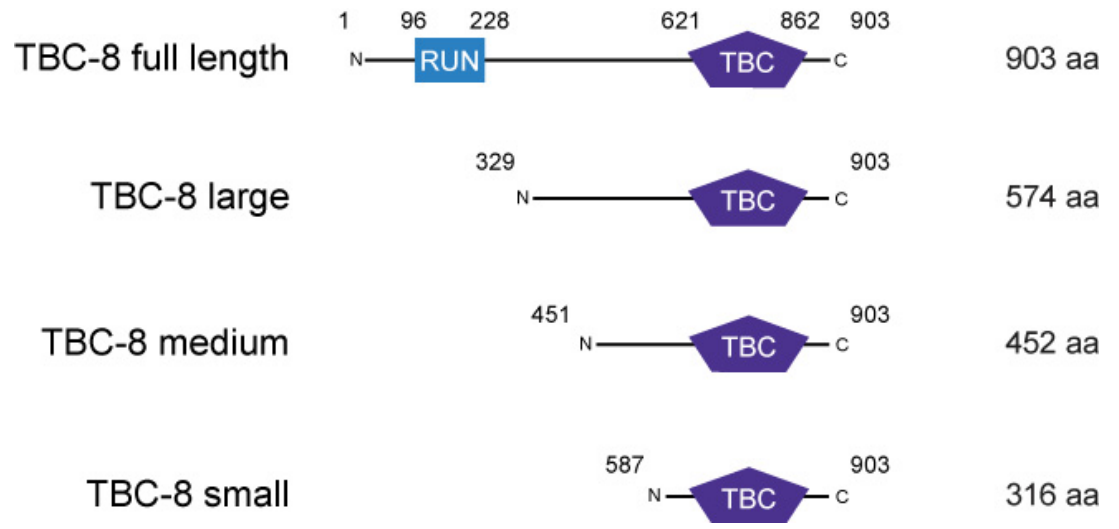

Figure IV.24. Representation of TBC-8 fragments that were used to test expression and solubility in $\boldsymbol{E}$. coli. Protein fragment sizes and lengths are depicted.

Table IV.1. Different constructs used for solubility test of recombinant TBC-8

\begin{tabular}{|c|c||c|c||}
\hline Construct & TBC-8 fragment & Fusion tag & $\begin{array}{c}\text { Predicted } \\
\text { GST-TBC-8 size }\end{array}$ \\
\hline \hline $\begin{array}{c}\text { pGST-tbc-8 full } \\
\text { length }\end{array}$ & $1-903$ aa & GST & $\sim 130 \mathrm{kDa}$ \\
\hline pGST-tbc-8 large & $329-903$ aa & GST & $\sim 94 \mathrm{kDa}$ \\
\hline pGST-tbc-8 medium & $451-903$ aa & GST & $\sim 80 \mathrm{kDa}$ \\
\hline pGST-tbc-8 small & $587-903$ aa & GST & $\sim 64 \mathrm{kDa}$ \\
\hline
\end{tabular}

For the expression and solubility test of all fragments, we transformed the according vectors into the E. coli BL21-CodonPlus(DE3)-RIL strain and tested different growth conditions. While expression was successful for all four TBC-8 fragments (Figure IV.25A), none of these proteins were found in the supernatant fraction, but were enriched in the pellet after sonication of E. coli, indicating that TBC-8 fragments were insoluble (Figure IV.25B). 
A Expression Test

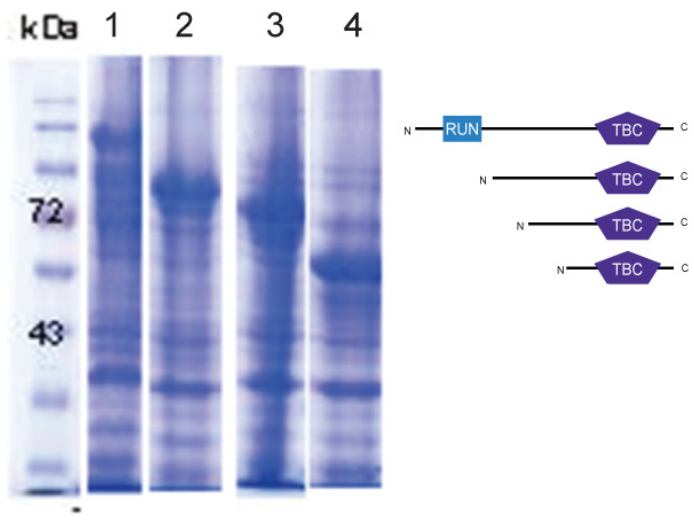

B Solubility Test

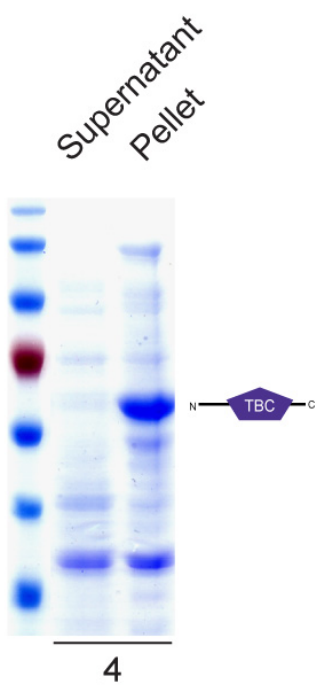

Figure IV.25.TBC-8 fragments are not soluble when expressed in E. coli. (A) Expression and (B) solubility test of TBC-8 fragments in E. coli. After expression, TBC-8 fragments (only TBC-8 small fragment is shown) were present in the pellet, indicating insolubility of TBC-8. This experiment was conducted by the author and Dr. Sabine König.

It is possible that posttranslational modifications were important for protein stability of TBC-8, which cannot be accomplished in bacteria. Therefore, we switched to the baculovirus expression system, which has been used for the production of properly post-translationally modified, recombinant proteins in insect cells. This work was done in cooperation with the Protein Expression and Purification Core Facility at the EMBL in Heidelberg (http://www.embl.de/pepcore/pepcore_services/index.html). For the expression of proteins in insect cells, we used His- and GST- tagged full length TBC-8 and the small TBC-8 (587-903 aa) fragment. Unfortunately, these protein fragments were also insoluble, when expressed in insect cells (Table IV.2). 
Table IV.2. Expression and solubility test of recombinant TBC-8 fragments used in insect cells.

\begin{tabular}{|ccc|}
\hline protein & overexpression & $\begin{array}{c}\text { small scale } \\
\text { purification }\end{array}$ \\
\hline $\begin{array}{c}\text { His-TBC-8 } \\
\text { His-TBC-8 } \\
(586-903 \text { aa })\end{array}$ & worked & insoluble \\
GST-TBC-8 & worked & insoluble \\
GST-TBC-8 & worked & insoluble \\
$(586-903$ aa $)$ & & insoluble \\
\hline
\end{tabular}

As a final attempt to obtain soluble TBC-8, we ordered a codon-optimized full-length TBC-8 for insect cells (from GeneArt), since the Protein Expression and Purification Core Facility at the EMBL had good experiences in improving the solubility of proteins by using codon-optimized proteins in the past. Unfortunately, using this $t b c-8$ codon-optimized gene did not result in obtaining soluble TBC-8 protein.

In summary, all our efforts to conduct an in-vitro GAP assay failed, since TBC-8 was not soluble. Therefore, we attempted to examine GAP activity of TBC-8 in vivo.

\section{IV.9 TBC-8 is a putative RAB-2 GAP}

Previously, RAB-2 was shown to localize to the Golgi (Sumakovic et al, 2009). Attempts to co-localize TBC-8 and RAB-2 were proven to be difficult, because when YFP-TBC-8 fusion proteins were co-expressed with mCherry-RAB-2 in motoneurons, we observed that RAB-2 was mainly cytosolic (Figure IV.26, cell bodies 2-4). However, in neurons where YFP-TBC-8 was not expressed due to the mosaic nature of extra-chromosomal arrays in C. elegans, a distinct Golgi staining of mCherry-RAB-2 was visible in these cell bodies (Figure IV.26, cell body 1). This is a strong indication that TBC-8, when overexpressed, inactivates RAB-2, which in turn would be redistributed from the Golgi to the cytosol. This co-localization experiment points to a possible GAP activity of TBC-8 towards RAB-2 in vivo and reinforces the idea that TBC-8 might be a RAB-2 GAP as evident from the genetic data. 

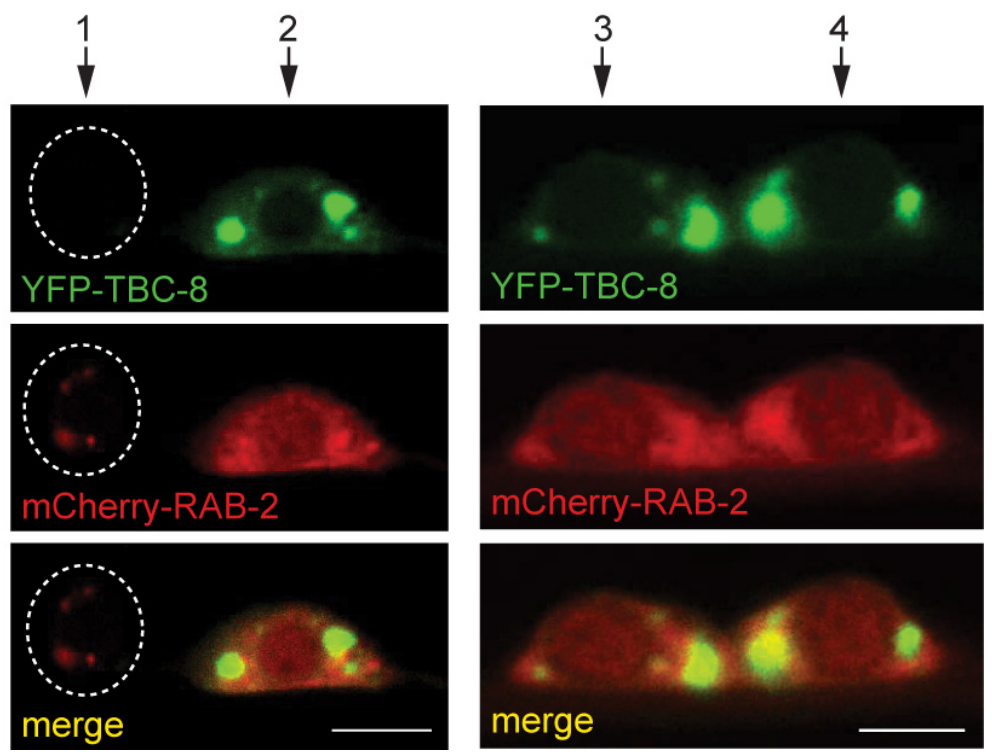

Figure IV.26. TBC-8 is a putative RAB-2 GAP. In motoneurons of the VNC, mCherryRAB-2 localizes to the Golgi (left cell body; cell boundary is highlighted by a dashed line). However, when YFP-TBC-8 is overexpressed, mCherry-RAB-2 is redistributed to the cytosol (cell bodies 2-4). Arrows one to four point to individual cell bodies of depicted motoneurons. Scale bars represent $3 \mu \mathrm{m}$. Source: (Hannemann et al, 2012).

It was previously demonstrated that TBC-domain containing Rab GAPs possess an essential arginine finger located within the TBC-domain, which is crucial for its catalytic GAP activity (Pan et al, 2006). When this catalytic arginine finger was mutated to alanine, the TBC-domain could still bind to its Rab partner but was unable to activate the intrinsic GAP activity of the Rab, which would lead to GTP hydrolysis (Pan et al, 2006). TBC-8 also contains a conserved arginine finger residue (R697) (Figure IV.27A) within the catalytic motif that is conserved among its orthologs in humans (SGSM1) and D. melanogaster (CG32506-PC) (Figure IV.27B).

A

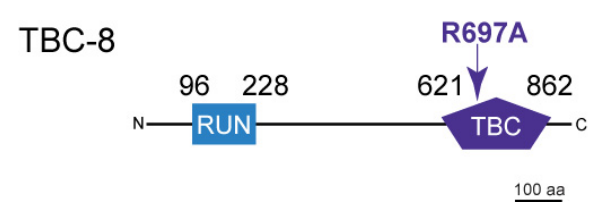

B

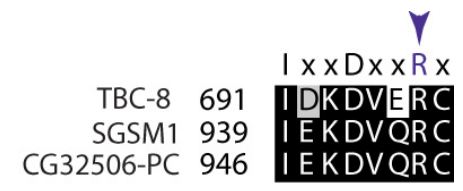

Figure IV.27. TBC-8 contains a conserved catalytic arginine finger within the TBCdomain. (A) Mutation of the catalytically active arginine (R697) to alanine within the TBCdomain renders the protein inactive. (B) Alignment of the catalytic motif of TBC-8 and its homologs in humans (SGSM1) and in Drosophila melanogaster (CG32506-PC) are shown. The arrowhead indicates the catalytic arginine residue necessary for GAP activity. Source: (Hannemann et al, 2012). 
If TBC-8 is the GAP specific for RAB-2, the interaction of both proteins should be detectable in the yeast two-hybrid system (Y2H). However, it was previously shown that interaction of wild type or GTP-bound Rab with its GAP was barely detectable using the Y2H system (Haas et al, 2005). The authors speculated that the interaction of the Rab GAP with its target GTP-bound Rab is transient and leads to a release of the Rab after triggering GTP hydrolysis. They solved this issue by introducing a mutation of the catalytic arginine finger within the TBC-domain of the GAP to alanine that made a detection of the Rab/GAP pair possible within the Y2H system (Haas et al, 2005).

In order to identify the RAB/TBC-8-GAP pair, we screened TBC-8 and the catalytically inactive TBC-8 (R697A) with all constitutively active, GTP bound $C$. elegans RAB proteins in a Y2H experiment (Figure IV.28). Interaction of TBC-8 with $\mathrm{RAB}$ proteins was captured by growth on histidine selection plates. As expected, RAB-2 (Q65L) interacted with the TBC-8 (R697A) and not with the wild type TBC-8 (Figure IV.28). This suggests that TBC-8 is a GAP for RAB-2 and the interaction between TBC-8 and RAB-2 is confined to the TBC-domain.

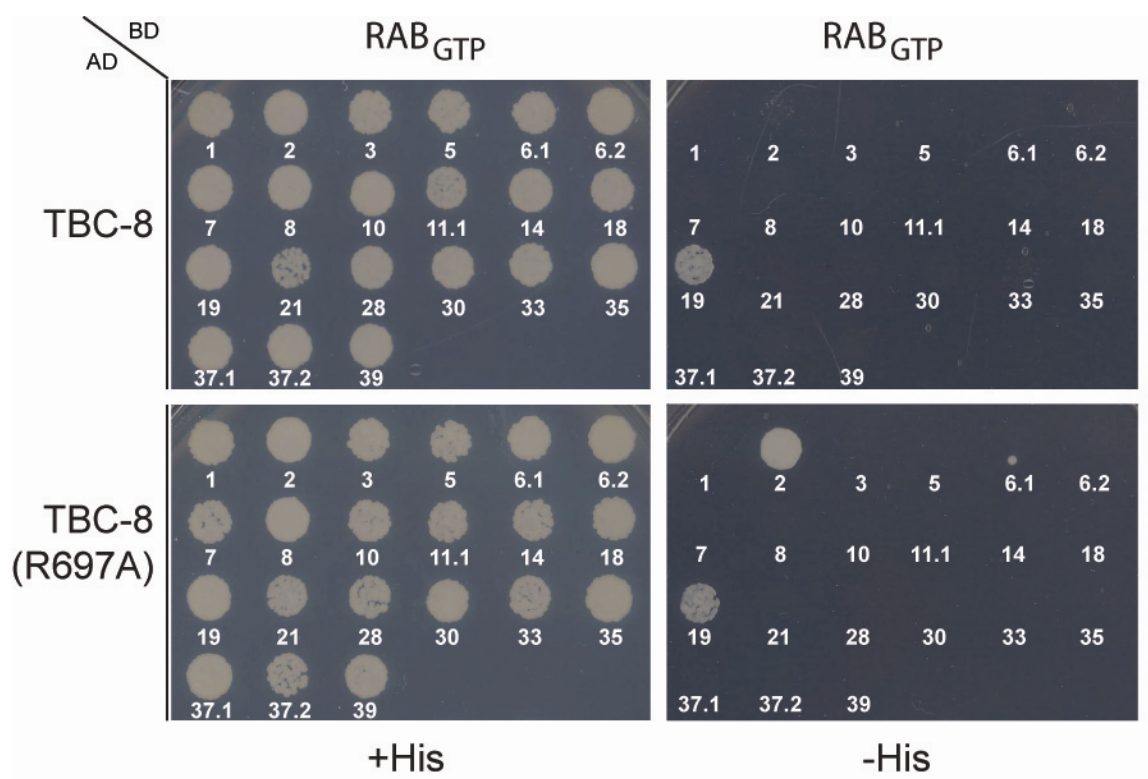

Figure IV.28. TBC-8 is a putative RAB-2 GAP. In a yeast two-hybrid assay, all C. elegans RABs in their constitutively GTP-bound form were tested against wild type TBC-8 (upper panel) and a catalytically inactive form of TBC-8 (R697A) (lower panel), respectively. Strikingly, RAB-2 (Q65L) interacted with TBC-8 (R697A) but not with wild type TBC-8, suggesting that TBC-8 is the GAP for RAB-2. Unlike RAB-2, RAB-19 (Q69L) interacted weakly with both forms of TBC-8, which makes it unlikely that TBC-8 is the GAP for RAB19 (Haas et al, 2005). AD, Gal4p DNA activation domain fusion; BD, Gal4p DNA binding domain fusion; His, histidine; $\mathrm{RAB}_{\mathrm{GTP}}$ : constitutively GTP-bound RAB GTPase. Source: (Hannemann et al, 2012). 
In this screen, we also detected a specific binding of the GTP-bound form of RAB-19 (Q69L) to TBC-8. However, in this case RAB-19 binds to both wild type TBC-8 and the catalytically inactive TBC-8 (R697A) in a GTP-dependent manner (Figure IV.29). This indicates that TBC-8 might be an effector and not a GAP of RAB-19.

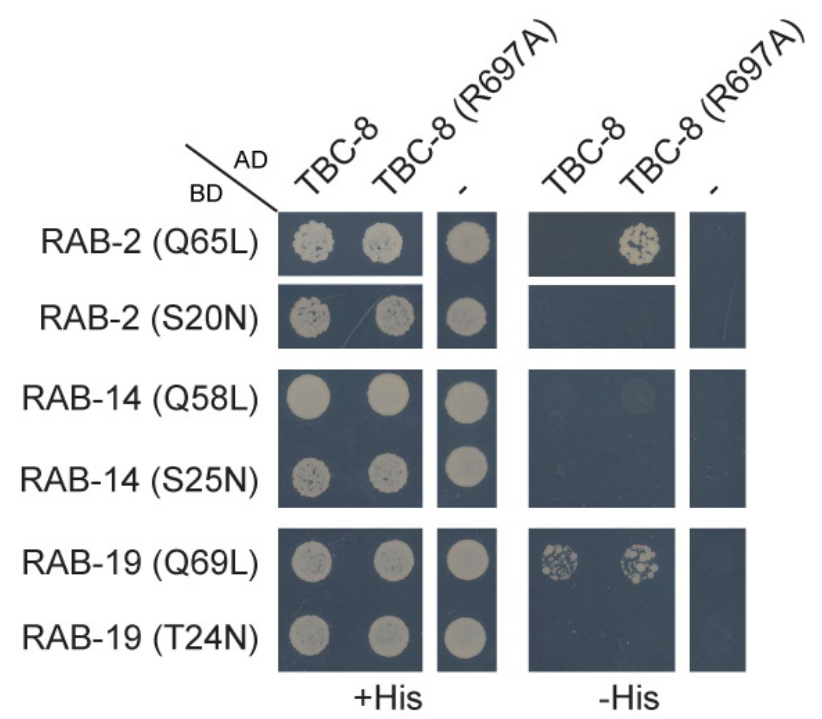

Figure IV.29. Interactions of TBC-8 with RAB-2 and RAB-19 are GTP-dependent. Constitutively active RAB-2 (Q65L) and RAB-19 (Q69L) interacted with TBC-8 whereas their dominant inactive forms [RAB-2 (S20N), RAB-19 (T24N)] did not. The closest paralog of RAB-2, RAB-14, did not show interaction with TBC-8 wild type or R697A in a yeast twohybrid analysis. AD, Gal4p DNA activation domain fusion; BD, Gal4p DNA binding domain fusion; His, histidine; $\mathrm{RAB}_{\mathrm{GTP}}$, constitutively GTP-bound RAB GTPase; “-“, empty vector pGADT7 was used for testing self-activation. Source: (Hannemann et al, 2012).

Furthermore, we could not detect defects in NLP-21-derived VENUS trafficking in rab-19(ok1845) deletion mutants (Figure IV.30) indicating that TBC-8 together with RAB-19 has another role besides DCV trafficking. 
NLP-21-VENUS fluorescence in dorsal nerve cord
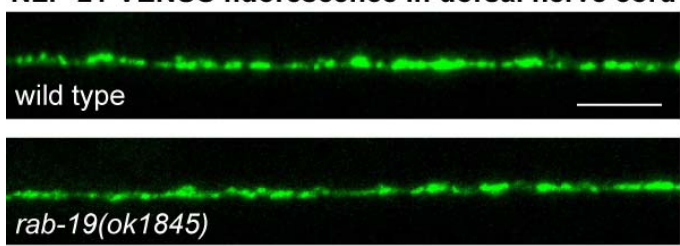

NLP-21-VENUS

in dorsal nerve cord

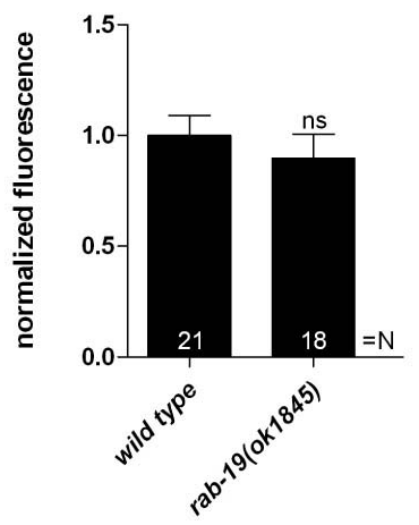

Figure IV.30. rab-19(ok1845) mutants do not display defects in DCV trafficking of the NLP-21-VENUS marker in the DNC. rab-19(ok1845) mutants have similar fluorescence levels of NLP-21-derived VENUS (89.83 $\pm 10.69 \%)$ in the DNC like wild type worms. Scale bar represents $5 \mu \mathrm{m}$. Error bars $=$ s.e.m. (ns, P > 0.05; Student's t-test). Source: (Hannemann et al, 2012).

All the data introduced in this chapter suggest that TBC-8 might be a putative neuronal GAP for the small GTPase RAB-2 in C. elegans. This hypothesis is mainly supported by the yeast two-hybrid analysis and by the co-localization studies of wild type TBC-8 and RAB-2 where we could show GAP activity of TBC-8 towards RAB2 in vivo. 


\section{Chapter 1}

\section{Discussion}

\section{V.1 TBC-8 is a putative neuron specific RAB-2 GAP}

Previously, we and others have shown that RAB-2 is involved in neuronal DCV maturation in C. elegans (Edwards et al, 2009; Sumakovic et al, 2009). A functional RAB-2 cycle is required to maintain specific (soluble and transmembrane) cargo in maturing DCVs. Due to the low intrinsic nucleotide exchange and hydrolysis rate of most of the Rab proteins, like RAB-2, additional proteins, GEFs and GAPs, are involved in regulation of Rab cycling (Segev, 2001; Zerial \& McBride, 2001). The identification of RAB-2 specific GAPs and GEFs would allow gaining insights into the spatio-temporal regulation of RAB-2 during DCV maturation. Therefore, we attempted to identify regulators for RAB-2, which are also involved in DCV maturation. In this study, we focused on the specific class of TBC-(Tre2/Bub2/Cdc16) domain containing Rab GAPs, because their catalytic domains are structurally conserved and functionally characterized (Pan et al, 2006). In addition, our previous study on RAB-2's role in DCV maturation was mainly conducted using dominant active alleles, rab-2 (n501) and rab-2 (n777). We hypothesized that eliminating a RAB-2 GAP, would render RAB-2 into a predominantly active state phenocopying the dominant active allele of rab-2. Therefore, using the previously established NLP-21-VENUS assay (Sieburth et al, 2007), we screened all TBC-domain containing RAB GAPs in C. elegans for a rab-2-like DCV maturation defect. From this screen, we identified TBC-8, an evolutionarily conserved RAB GAP. 
We demonstrated that the GAP activity of TBC-8 is crucial for its role in regulating DCV maturation because a mutation in its catalytic motif "IxxDxxR" within the TBC-domain (R697A), unlike the wild type variant, failed to rescue the DCV phenotype in $t b c-8$ deletion mutants. These data suggested that TBC-8 must act on a substrate Rab.

Expression pattern analysis of $t b c-8$ using a transcriptional reporter construct revealed that $t b c-8$ was specifically expressed in neurons. The strong neuronal expression along with the observed DCV maturation in $t b c-8$ deletion mutants, suggested that TBC-8 is a neuron specific GAP. Given the similarity in DCV maturation phenotypes of tbc-8 and $r a b-2$ mutants and the overlapping expression patterns of both genes, we postulated that TBC-8 may be the GAP for RAB-2.

RAB-2 has previously been reported to also function in several trafficking events within non-neuronal tissues of C. elegans (Chun et al, 2008; Lu et al, 2008; Mangahas et al, 2008). If TBC-8 were the only GAP for RAB-2, then $t b c-8$ mutants would have similar trafficking defects in these non-neuronal tissues. Analysis of the removal of apoptotic cell corpses in the germ line and post-endocytic trafficking in coelomocytes revealed that TBC-8 does not regulate $\mathrm{RAB}-2$ activity these tissues. These results indicate other GAPs exist that regulate RAB-2 activity in the germ line and in coelomocytes. In addition, we cannot exclude that in different subtypes of neurons, RAB-2 function may be regulated by GAPs other than TBC-8.

Unfortunately, due to the inability of obtaining soluble TBC-8 when expressed in bacteria or insect cells, we were unable to show GAP activity towards RAB-2 in vitro. Further efforts should be made to obtain soluble TBC-8 by expressing TBC-8 (or TBCdomain fragments) by using cell-free "in vitro translation systems”. Another possibility could be to purify TBC-8 from mammalian cell culture, since we could show that TBC-8 seems to be soluble (at least in some extent) in HEK293 cells during coimmunoprecipitation experiments (this study and our unpublished data). GAP activity assays could be performed with these TBC-8-containing cell lysates and with TBC-8-free cell lysates as control.

Despite the inability of performing a GAP assay in vitro at the moment, three findings indicate that TBC-8 act as a RAB-2 specific GAP in vivo. First, the analysis of the two double mutants of $t b c-8$ and $u n c-108 / \mathrm{rab}-2$ revealed that both proteins are 
involved in the same pathway. Second, in the yeast two-hybrid system, the GTP-bound form of RAB-2 interacted with TBC-8 but only if the TBC-domain is catalytically inactive. Previously, it was shown that an exchange of the catalytic arginine to alanine within the TBC-domain could be used to detect interaction of a GAP with its cognate Rab (Haas et al, 2005). Third, TBC-8 influences the membrane localization of its binding partner RAB-2 when over-expressed in neurons. It was shown that active GTP-bound RAB-2 localizes to discrete puncta at the Golgi apparatus while inactive GDP-bound RAB-2 is mainly cytosolic (Sumakovic et al, 2009). However, when TBC-8 was overexpressed, RAB-2 was redistributed to the cytosol. Such redistribution of a Rab upon expression of its GAP has been previously used as an indication to screen for functional Rab/GAP pairs (Ishibashi et al, 2009). All these results strongly suggest that TBC-8 is indeed a likely RAB-2 GAP in neurons.

\section{V.2 TBC-8 is conserved throughout evolution}

Via BLAST searches we could show that TBC-8 is conserved throughout evolution. There are two possible orthologs in mammals: SGSM1 (small G protein signaling modulator 1) also called RUTBC2 (RUN and TBC1 domain containing 2) (Yang et al, 2007) and SGSM2/RUTBC1 (Nottingham et al, 2011; Yang et al, 2007). Although TBC-8 shares domain structure with SGSM1 and SGSM2, it is more similar to SGSM1 based on sequence alignments. The TBC-domain of SGSM2 is about 162 aa longer than the TBC-domain of TBC-8, leading to a higher gap penalty when both proteins were aligned. However, multiple splice variants can be found for both SGSM1 and SGSM2. Thus, we cannot exclude the possibility that one of those variants is even more closely related to TBC-8.

Whereas SGSM2 and SGSM3 were ubiquitously expressed, SGSM1 was predominantly expressed in brain, heart, and testis (Yang et al, 2007). It was demonstrated that SGSM1 localized to the TGN in neuronal cell culture (Yang et al, 2007). Furthermore, SGSM1 weakly interacted with different Rab proteins in co-IP experiments; however, interactions with specific domains of SGSM1 were not studied 
(Yang et al, 2007). In addition, SGSM1 bound to members of the Rap GTPase family in co-IP experiments (Yang et al, 2007). The authors postulated a role of SGSM family proteins in neuronal, intracellular transport (Yang et al, 2007).

Recently, SGSM2/RUTBC1 has been found as a Rab9 effector (Nottingham et al, 2011). Furthermore, functional GAP activity of SGSM2 towards Rab32 and Rab33 has been demonstrated in vitro (Nottingham et al, 2011). However, the C. elegans genome does not encode a Rab9 homolog, nor could we detect any interaction between TBC-8 and RAB-33 or GLO-1, the C. elegans Rab32 homolog. Based on the fact that Drosophila contains two SGSM1/2 homologs, it is likely that C. elegans has lost the SGSM2 homolog along with Rab9 and Rab32 during evolution. Thus, we reason that SGSM1 is more closely related to TBC-8, consistent with their strong neuronal expression.

TBC-8 and its homologs SGSM1/2 also contain an N-terminal RUN (RPIP8/UNC-14/NESCA) domain, which consists of $\alpha$-helices (Callebaut et al, 2001; Kukimoto-Niino et al, 2006). It is proposed that RUN domains are required to facilitate protein-protein interactions, specifically with small GTPases of the Rab and Rap family (Janoueix-Lerosey et al, 1998; Recacha et al, 2009; Yang et al, 2007). Consistently, a SGSM1 fragment containing the RUN domain co-precipitated with both Rap1 and Rap2 in a co-IP experiment (Yang et al, 2007). We have also tested all C. elegans Rab and Rap proteins with TBC-8 and only RAB-19, besides RAB-2, interacted with TBC-8, whereas no binding of the RAP family with TBC-8 was observed within the yeast two-hybrid system, suggesting that the interaction between the RUN domain and Rap GTPases might not be conserved (Appendix, Figure XV.2).

\section{V.3 TBC-8 regulates DCV maturation}

By utilizing the NLP-21-VENUS DCV assay we could demonstrate that TBC-8 is involved in DCV maturation, since $t b c-8(t m 3802)$ deletion mutants had similar defects in NLP-21-VENUS trafficking as unc-108/rab-2 mutants. Similar to the defects seen with NLP-21-VENUS, the FRMF-like neuropeptide FLP-3-VENUS was also affected by $t b c-8$ 
deletion and showed decreased VENUS levels at the DNC in these mutants compared to wild type. As in the case of unc-108/rab-2 mutants (Edwards et al, 2009), we did not detect defects in the trafficking of the insulin-like neuropeptide, INS-22-VENUS. Unlike NLP-21 and FLP-3, INS-22 lacks proprotein convertase 2 (PC2) cleavage sites (Edwards et al, 2009; Pierce et al, 2001). Due to the presence of these PC2 sites, proneuropeptides are cleaved into shorter, bioactive fragments $(\mathrm{Li}, 2005)$ leading to the simultaneous aggregation of the processed neuropeptides and the liberation of the C-terminally tagged soluble YFP in the DCVs (Edwards et al, 2009) (Figure II.15). This is not the case with INS-22-VENUS because the VENUS-tag is not cleaved from the proneuropeptide INS22 during the maturation process within DCVs, so that the VENUS-tag is aggregated together with INS-22 and other neuropeptides within the insoluble core of DCVs (Pierce et al, 2001; Sieburth et al, 2007). These data suggest that it is mainly the soluble cargo that is lost in $t b c-8$ mutants, as observed also in the unc-108/rab-2 mutants. Since soluble VENUS cargo is lost in tbc-8 and unc-108/rab-2 mutants, the question arises whether neuropeptides are also affected by mutations in $t b c-8$ and unc-108/rab-2. Different data indicate that neuropeptides are per se not affected in unc-108/rab-2 mutants. Mass spectrometry analysis showed that all detected neuropeptides were fully processed in unc108/rab-2 mutants (Sumakovic et al, 2009). Furthermore, immunostaining of processed FRMF-amide neuropeptides in the DNC of unc-108/rab-2 mutants revealed that similar levels of these neuropeptides were present in the DNC in unc-108/rab-2 mutants compared to wild type worms (Edwards et al, 2009). This is consistent with EM analysis showing wild type numbers of DCVs at synapses in both, tbc-8 and unc-108/rab-2 mutants (Edwards et al, 2009; Sumakovic et al, 2009). Moreover, immunostaining of FMRF-amide neuropeptides also indicates that neuropeptide processing is unaffected in unc-108/rab-2 mutants (Edwards et al, 2009). It could be shown before that disturbed neuropeptide processing affected the binding of the antibody with FRMF-amide neuropeptides, resulting in low antibody signals (Jacob \& Kaplan, 2003).

Surprisingly, we did not detect any differences in the axonal fluorescence levels of the DCV integral membrane protein, IDA-1-GFP, in $t b c-8(t m 3802)$ mutants. This result indicates that transmembrane cargos might not be affected by tbc-8 deletion. However, in unc-108/rab-2 mutants, the axonal fluorescence of the transmembrane cargo IDA-1-GFP 
was strongly decreased (Edwards et al, 2009). This suggests that both soluble and transmembrane cargos are lost from DCVs in unc-108/rab-2 mutants (Edwards et al, 2009), unlike in $t b c-8$ mutants. This discrepancy in transmembrane cargos being affected in unc-108 mutants but not in tbc-8 mutants implies that in neurons, another GAP than TBC-8 may exist at the site where RAB-2 functions to retain transmembrane cargos. Despite this difference, the axonal DCV numbers were unchanged by rab-2/unc-108 or $t b c-8$ mutations compared to wild type worms as determined by electron microscopy [this study; (Edwards et al, 2009; Sumakovic et al, 2009)].

All these data demonstrate that TBC-8 contributes together with RAB-2 in the retention of specific (soluble) cargo in DCVs during the maturation process. Since a blockade of the endosomal delivery by over-expression of constitutively active GTPbound RAB-5 (Q79L) could rescue the loss of soluble NLP-21-derived VENUS, TBC-8 and RAB-2 are likely involved in cargo sorting processes during maturation at the Golgiendosomal interface.

\section{V.4 Unlike unc-108/rab-2 mutants, tbc-8 deletion mutant worms display no movement defects}

Despite the similarities observed in DCV maturation, there is an obvious difference in behavioral phenotype in both tbc-8(tm3802) and unc-108/rab-2(n501) mutants. tbc-8(tm3802) deletion mutants display no defects in locomotion behavior, whereas unc-108/rab-2 mutants are strongly impaired in movement (Edwards et al, 2009; Sumakovic et al, 2009).

There are several possible explanations why both mutants show different locomotion phenotypes.

First, $t b c-8$ may not be expressed in all subtypes of neurons that are necessary to coordinate locomotion, whereas unc-108/rab-2 is broadly expressed in (all) neurons and other tissues (Chun et al, 2008; Sumakovic et al, 2009). Interestingly, it was shown that the locomotory defect in unc-108/rab-2 mutants could only be rescued by expressing unc-108 under a pan-neuronal promoter (psnb-1), but not when unc-108 was expressed 
under the unc-17 promoter, which is expressed in a specific subtype of motoneurons, the cholinergic motoneurons (Chun et al, 2008). This indicates that unidentified subtypes of neurons exist, which are "master" regulators of locomotion. Therefore, we hypothesize that TBC-8 does not function in all the specific subtypes of neurons where locomotion is coordinated, resulting in wild-type-like movement observed in $t b c-8$ deletion mutants.

Secondly, in the absence of $t b c-8$ in some neurons, another GAP might redundantly substitute for TBC-8 function. This hypothesis is further supported by the fact that the transmembrane protein IDA- 1 is unaffected by $t b c-8$ deletion, but is affected by unc-108/rab-2 mutations, suggesting that another GAP must exist in neurons.

Thirdly, in the absence of TBC-8, it is possible that the low intrinsic activity of RAB-2 might partly rescue some of the phenotypes in $t b c-8$ mutants that are observed in unc-108/rab-2 mutants.

Fourthly, an unknown transmembrane factor, which is normally present on DCVs could be lost in unc-108/rab-2 mutants during DCV maturation and thus be responsible for the uncoordinated phenotype. This transmembrane factor could still be present in $t b c$ 8 mutants since no defects in IDA- 1 trafficking were observed in $t b c-8$ mutants.

Collectively, our results indicate that, despite differences in behavioral phenotypes, tbc-8(tm3802) and unc-108/rab-2(n501) dominant active mutants share similar defects in DCV maturation, reiterating the idea that TBC-8 is a putative GAP for RAB-2 in neurons (Figure V.1). 


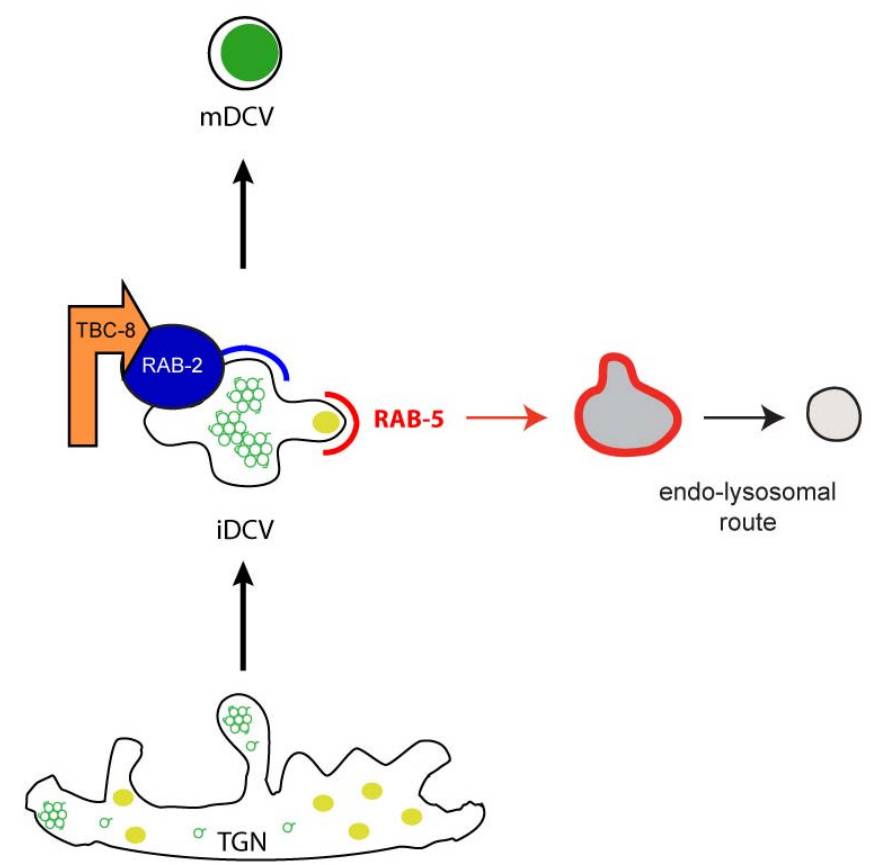

Figure V.1. Schematic representation of neuronal DCV maturation in C. elegans. The maturing DCV compartment might exhibit different domains on its surface, which are important for specific function ensured by a particular set of proteins (e.g. RAB-2 domains). The RAB-2 GAP, TBC-8, required for deactivation of RAB-2, is also involved in DCV maturation. From our previous work, we predict that RAB-5 might form a domain from which mis-sorted cargo is removed to the endo-lysosomal pathway [our unpublished data; (Sumakovic et al, 2009)]. Source: picture modified from (Tooze et al, 2001), with permission from Elsevier.

This study raises now additional questions. How is TBC-8 activity regulated? How do RAB-2 and TBC-8 play together in DCV maturation? Does TBC-8 have additional roles in neurons? Since TBC-8 interacted with active GTP-bound RAB-19 in a Y2H assay, but rab-19(ok1845) deletion mutants did not display defects in NLP-21VENUS trafficking, it is likely that TBC-8 together with RAB-19 might act in another DCV-independent pathway that has to be identified. Further studies have to be conducted to answer all these open questions.

The next chapters provide a deeper insight into DCV maturation by identifying new players involved in this process. Furthermore, we highlight on additional possible roles that TBC-8 might have during DCV maturation. 


\section{Chapter 2}

\section{Role of a RAB-2 effector complex during dense-core vesicle maturation}

\section{Specific Introduction}

\section{VI.1 Role of RAB-2 and its effector RIC-19 in DCV maturation in C. elegans}

Previously, our lab and others have demonstrated that the small GTPase RAB-2 is crucial for DCV maturation in C. elegans (Edwards et al, 2009; Sumakovic et al, 2009). These studies suggested a role of RAB-2 for the retention of soluble and transmembrane cargos within maturing DCVs. Loss of RAB-2 function resulted in mis-sorting of cargo into the endosomal-lysosomal pathway (Edwards et al, 2009; Sumakovic et al, 2009). Colocalization studies revealed that RAB-2 is localized to the Golgi complex and cannot be detected at synapses, indicating that RAB-2 function is mainly restricted to the neuronal cell body (Sumakovic et al, 2009). Furthermore, EM analysis discovered no changes in DCV numbers at synapses, indicating that RAB-2 might not be involved in the formation of DCV at the Golgi (Sumakovic et al, 2009).

It was shown that the C. elegans ICA69 homolog, RIC-19, is an effector of RAB2 (Sumakovic et al, 2009). The localization of RIC-19 is dependent on RAB-2. Only GTP-bound RAB-2 recruits RIC-19 to Golgi membranes, whereas GDP-bound RAB-2 causes cytosolic distribution of RIC-19 in neurons (Sumakovic et al, 2009). Interestingly, 
ric-19 mutants displayed similar DCV maturation defects to unc-108/rab-2 mutants. By utilizing their BAR (Bim/amphiphysin/Rvs) domains, RIC-19 and its human homolog ICA69 are predicted to bind to curved membranes found on vesicular structures (Gallop \& McMahon, 2005; Habermann, 2004; Zimmerberg \& McLaughlin, 2004). Therefore, RAB-2 together with RIC-19 are predicted to create and stabilize membrane domains on maturing DCVs that might be important to maintain specific cargo within these vesicular structures (Figure VI.1) (Sumakovic et al, 2009).

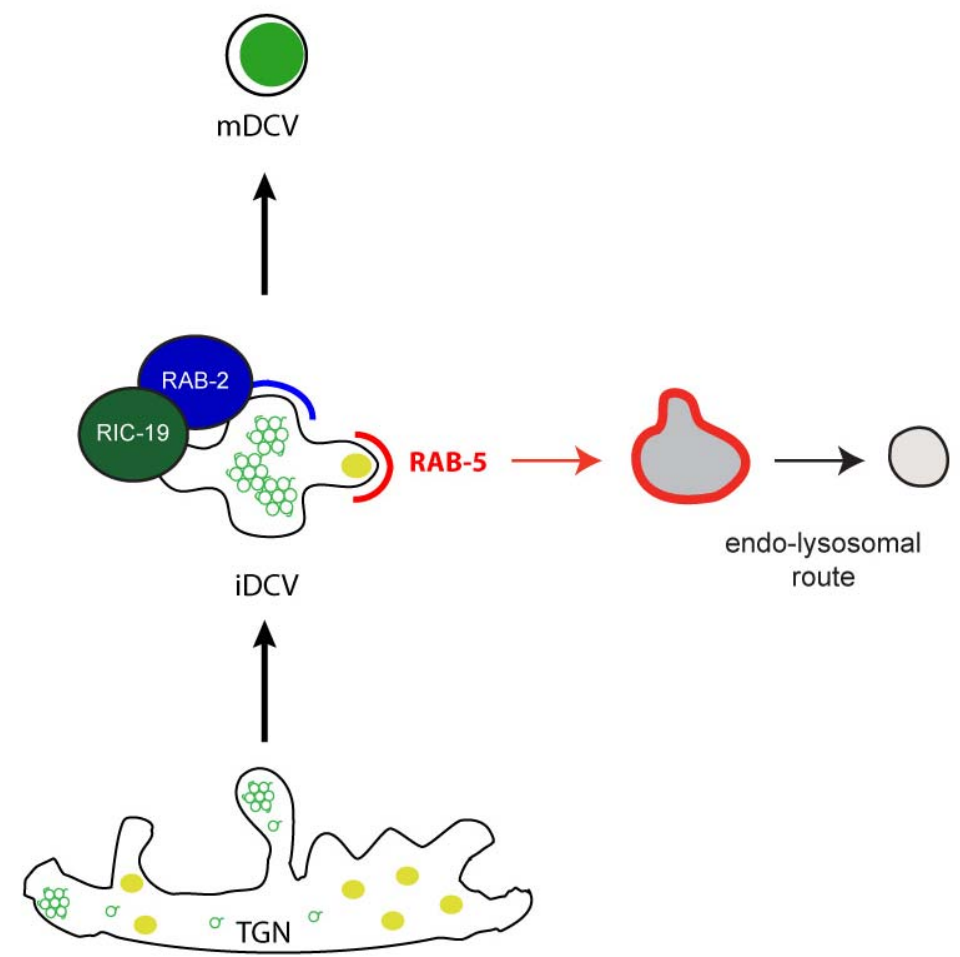

Figure VI.1. RAB-2 and its effector, RIC-19 are involved in DCV maturation. Activated, GTP-bound RAB-2 recruits its effector, RIC-19 to membranes. This interaction is required to prevent loss of cargo during DCV maturation into the endosomal-lysosomal pathway. Source: picture adapted from (Tooze et al, 2001), with permission from Elsevier. 


\section{VI.2 Aims of this study}

Based on the impaired locomotion, unc-108/rab-2 mutant alleles were isolated in C. elegans. When unstimulated, these alleles hardly moved and displayed an "unmotivated" movement phenotype, which is caused by disturbed DCV signaling (Edwards et al, 2009; Sumakovic et al, 2009). The characteristic "unmotivated" locomotion largely resembles that of unc-31/CAPS mutants, which is indicative to mutants in the DCV signaling pathway. To find new factors involved in RAB-2 dependent DCV maturation, our collaborator Michael Ailion (University of Washington, USA) conducted a screen for mutants that display an unmotivated locomotion. This screen identified mutants in 6 novel genes (M. Ailion, unpublished data). Two of these genes were characterized in this chapter. We investigated how these molecules function together with RAB-2 during DCV maturation. Moreover, we demonstrate a possible connection of the known RAB-2 effector, RIC-19, with the RAB-2 GAP, TBC-8 (identified in chapter 1). Furthermore, we provide possible explanations how all factors cooperate to either regulate the small GTPase, RAB-2 or to facilitate RAB-2 functions in neuronal DCV maturation in C. elegans.

This project was conducted in cooperation with Dr. Michael Ailion, (University of Washington, USA). The results presented in chapter 2 were performed by the author of this thesis, unless otherwise stated as “M. Ailion, unpublished data”. 


\section{Chapter 2}

\section{Results}

\section{VII.1 TBC-8 interacts with the RAB-2 effector RIC-19/ICA69}

Previously, it has been shown that the BAR-domain (Bin/amphiphysin/Rvs) containing protein, RIC-19/ICA69, is an effector of RAB-2 (Buffa et al, 2008; Sumakovic et al, 2009), since GTP-bound, activated RAB-2 recruited RIC-19 to Golgi membranes (Sumakovic et al, 2009). As highlighted in chapter 1, the TBC-domain containing protein, TBC-8, is likely to be a RAB-2 specific GAP in C. elegans neurons. To determine whether TBC-8 and RIC-19 act in the same pathway during RAB-2 dependent DCV maturation we constructed a tbc-8(tm3802); ric-19(ok833) double mutant and analyzed this mutant for loss of the DCV marker NLP-21-VENUS from maturing DCVs. As shown in Figure VII.1, NLP-21-derived VENUS fluorescence in the dorsal nerve cord (DNC) was decreased in $t b c-8$ mutants by $71.61 \pm 3.53 \%$, in $t b c$ 8(tm3802); ric-19(ok833) by $78.25 \pm 1.90 \%$, whereas ric-19(ok833) single mutants showed a weaker decrease in fluorescence by $57.44 \pm 4.55 \%$. Similar results were observed for the NLP-21-derived VENUS fluorescence in the coelomocytes (Figure VII.1B). These findings suggest that TBC-8 and RIC-19 act in the same pathway to prevent the loss of soluble cargo during DCV maturation. 
A NLP-21-VENUS fluorescence in dorsal nerve cord
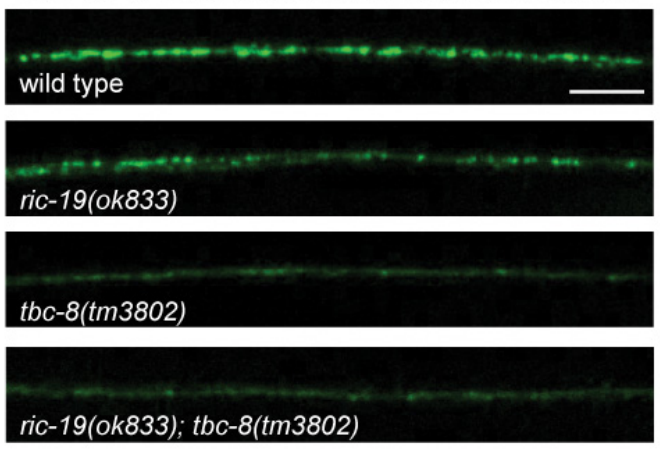

B

NLP-21-VENUS fluorescence

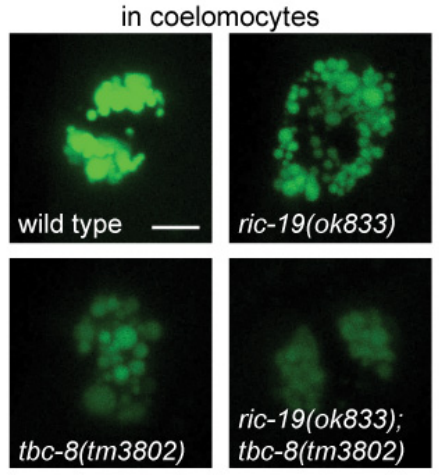

NLP-21-VENUS in dorsal nerve cord

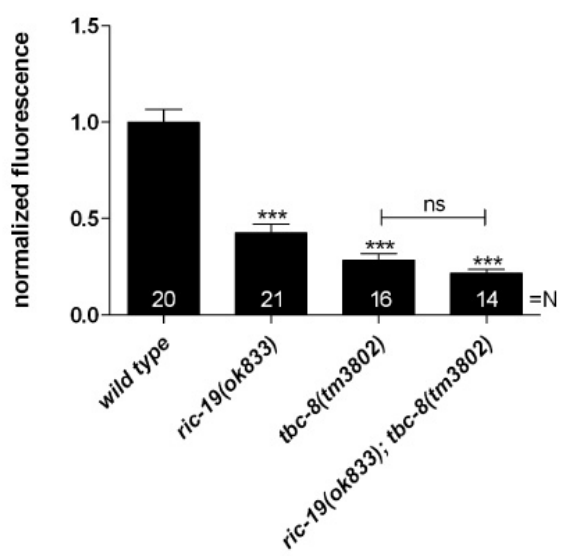

NLP-21-VENUS in coelomocytes

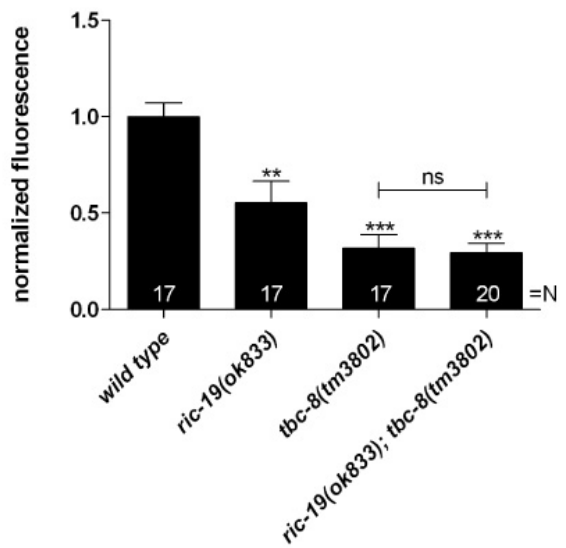

Figure VII.1. The RAB-2 effector, RIC-19/ICA69 genetically interacts with the putative RAB-2 GAP, TBC-8. NLP-21-VENUS analysis of the single mutants ric-19(ok833), tbc$8(t m 3802)$ and the double mutant of both in the DNC (A) and the coelomocytes (B) revealed that RIC-19 and TBC-8 act in the same genetic pathway. Scale bar represents $5 \mu \mathrm{m}$. Error bars $=$ S.E.M. (***, P $<0.0001 ; * *, \mathrm{P}<0.001$, Student's t-test). Source: (Hannemann et al, 2012).

We reasoned that overexpression of the GAP, TBC-8, should cause inactivation of RAB-2 in neurons, which should lead to a completely cytosolic localization of the effector RIC-19. Unexpectedly, when tagRFP-TBC-8 was co-expressed with RIC-19YFP in neurons, both proteins co-localized into huge cytosolic puncta in the cell bodies of motoneurons (Figure VII.2, upper panel). This co-localization of the putative RAB-2 GAP and the RAB-2 effector suggested that both proteins might interact and form a 
complex that localizes to intracellular membranes. The extent of co-localization and the size of the puncta indicate that TBC-8 and RIC-19 strongly drive the membrane recruitment of the other. Interestingly, this cooperative membrane localization of TBC-8 and RIC-19 forced by overexpression is independent of RAB-2, since it can also be observed in unc-108/rab-2(nu415) null mutant backgrounds (Figure VII.2, lower panel).

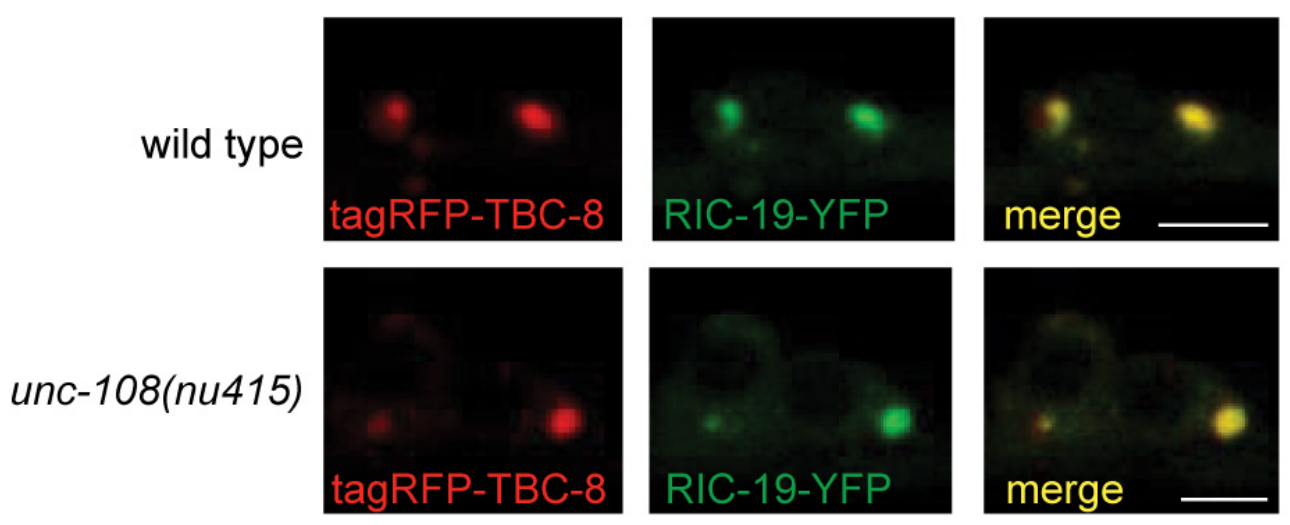

Figure VII.2. TBC-8 and RIC-19 co-localize in neurons. Overexpression of tagRFP-TBC-8 and RIC-19-YFP led to a full co-localization of both proteins in neurons. This co-localization of TBC-8 and RIC-19 is RAB-2-independent, since it was also detected in a unc-108/rab-2(nu415) null mutant background. Scale bar represents $3 \mu \mathrm{m}$. Source: (Hannemann et al, 2012).

The co-localization and the cooperative membrane recruitment strongly suggested that RIC-19 and TBC-8 might directly interact. We tested this idea by a coimmunoprecipitation (co-IP) experiment of RIC-19 and TBC-8 in HEK293 (Human Embryonic Kidney 293) cells as well as by a yeast two-hybrid analysis (Y2H) (Figure VII.3). Both experiments revealed that there is a direct interaction between the RAB-2 effector, RIC-19, and the putative RAB-2 GAP, TBC-8. Moreover, we confirmed by coIP and $\mathrm{Y} 2 \mathrm{H}$ analysis that an N-terminal fragment of TBC-8 (1-597 aa) containing the RUN-domain was sufficient for binding to RIC-19 (Figure VII.3). Furthermore, we observed interaction between TBC-8 and the human RIC-19 ortholog, ICA69, by Y2H analysis (Figure VII.3B). Thus, the interaction between TBC-8 and RIC-19 seems to be evolutionarily conserved. 
A

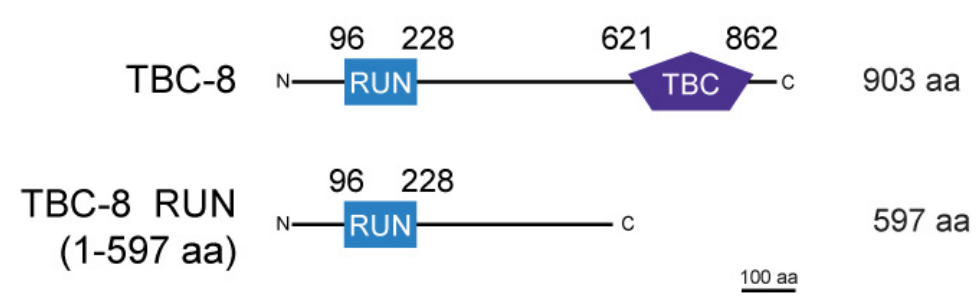

B

\section{yeast two-hybrid}

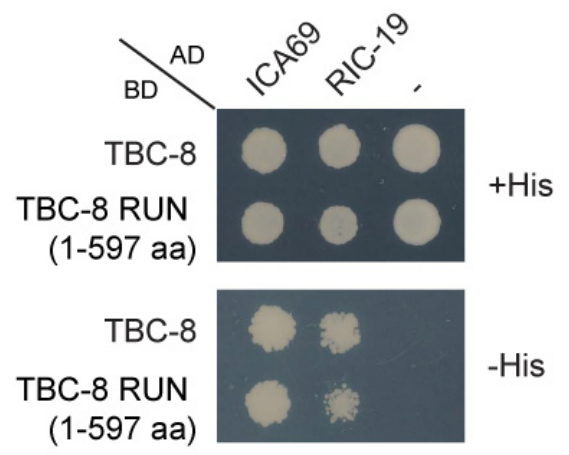

C co-immunoprecipitation

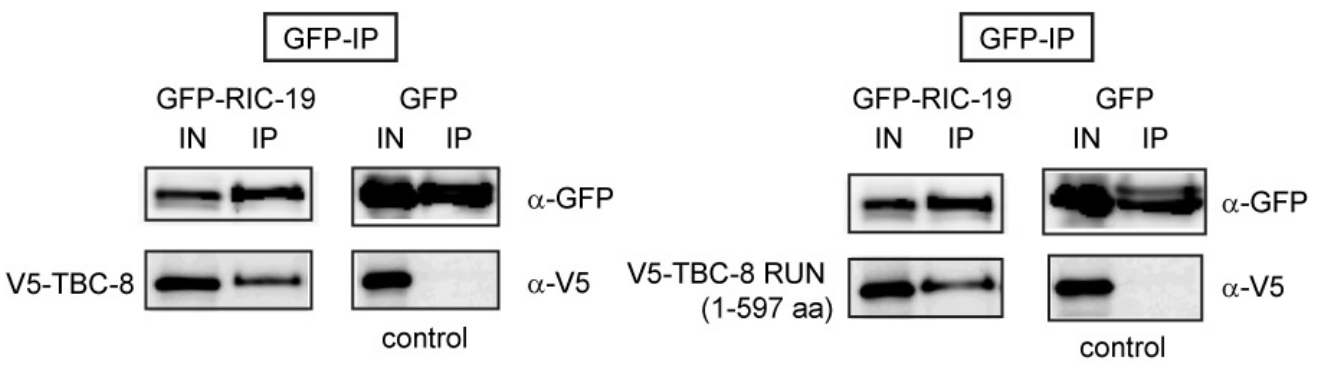

Figure VII.3. The RAB-2 effector, RIC-19, and the putative RAB-2 GAP, TBC-8, interact in yeast two-hybrid analysis (Y2H) and co-immunoprecipitation (co-IP) (A) Schematic representation of TBC-8 constructs: full-length and RUN domain (1-597 aa) used for Y2H (B) and co-IP (C). Y2H: RIC-19 and its human ortholog, ICA69, interacted with full length TBC-8 and TBC-8 RUN domain (1-597 aa) suggesting conservation of this interaction. Co-IP: HEK293 cells were co-transfected with constructs expressing GFP-tagged RIC-19 (or GFP alone as control) and V5-TBC-8 [full length or RUN domain (1-597 aa)]. An anti-GFP antibody was used to precipitate GFP-RIC-19 or GFP as control (GFP-IP). Interactions were visualized on Western blots. AD, Gal4p DNA activation domain fusion; $\mathrm{BD}$, Gal4p DNA binding domain fusion; His, histidine; IN, Input; IP, immunoprecipitation; “-“", empty vector pGADT7 was used for testing self-activation. Source: (Hannemann et al, 2012). 
Any attempt to shorten the TBC-8 RUN-domain construct resulted in a loss of the interaction with RIC-19 (Figure VII.4). Since the minimal interaction fragment of TBC-8 contains regions in addition to the RUN-domain, it is likely that the interdomain fragment from 329-597 aa provides additional binding motifs that are necessary along with the RUN-domain for RIC-19 binding.
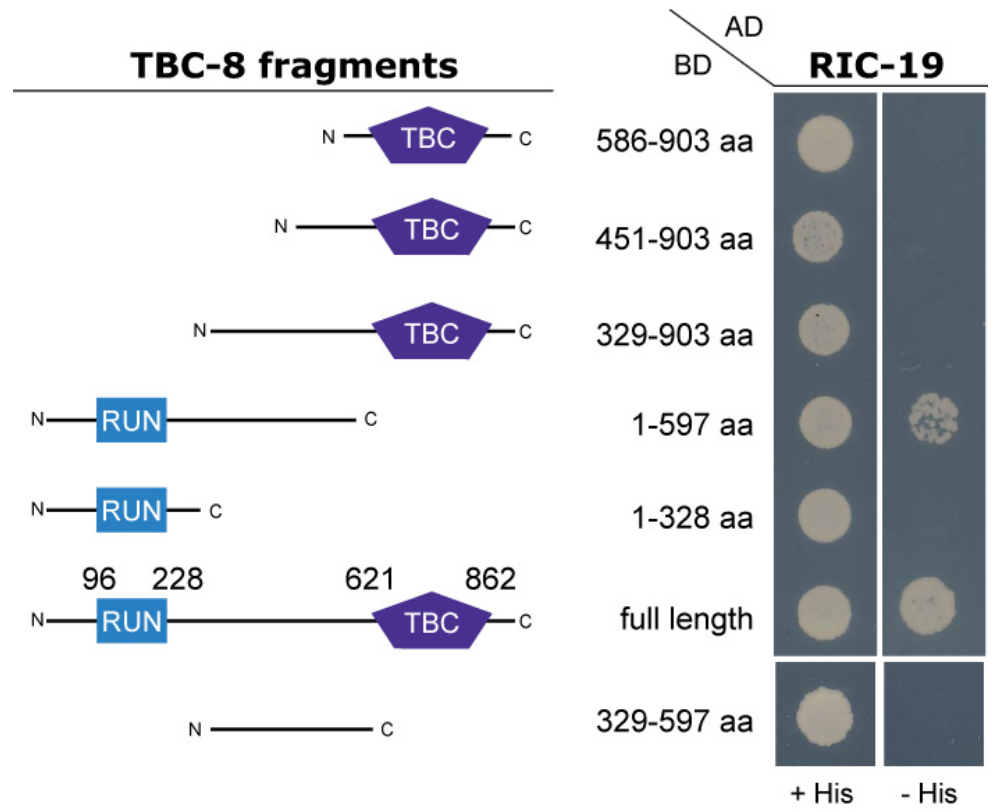

Figure VII.4. Domain mapping of TBC-8 for interaction with RIC-19. Full-length TBC-8 as well as a TBC-8 construct harboring the RUN-domain (1-597 aa) interacted with RIC-19 in a yeast two-hybrid analysis. Attempts to shorten the TBC-8 RUN-domain resulted in a loss of the interaction. AD, Gal4p DNA activation domain fusion; BD, Gal4p DNA binding domain fusion; His, histidine.

Our data suggest that active GTP-bound RAB-2 might recruit its own GAP via its effector RIC-19. However, this hypothesis has to be confirmed in further studies. 


\section{VII.2 The novel factor, RUND-1, involved in DCV function}

When unstimulated, unc-108/rab-2 mutant animals show a slow locomotion and hardly move. This characteristic "unmotivated" phenotype, which largely resembles that of unc-31/CAPS mutants, is indicative to mutants in the RAB-2 dependent DCV maturation pathway. To find new factors involved in RAB-2 dependent DCV maturation, our collaborator Michael Ailion (University of Washington, USA) conducted a screen for mutants that display an unmotivated locomotion. This screen identified mutants in 6 novel genes (M. Ailion, unpublished data). One of the identified genes was rund1/T19D7.4. Two point mutation rund-1 alleles were identified (M. Ailion, unpublished data). In addition, the Japanese knock out consortium had generated a deletion allele of rund-1, tm3622, which was mainly used in this study (www.wormbase.org). rund1(tm3622) represents a deletion allele removing exon 7,8 and the first part of exon 9 (Figure VII.5A). However, since the deletion starts within intron 6, the exact fate of the partially translated product is still unclear and has to be further studied.

A

rund-1

Length 3134 bases

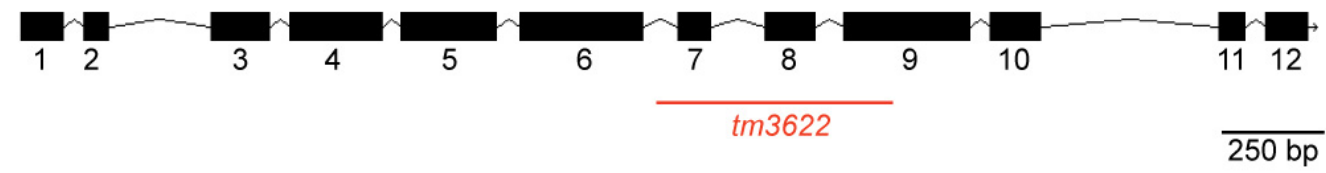

B

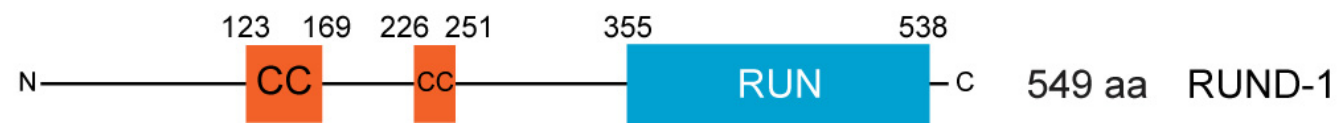

Figure VII.5. Schematic representation of the gene rund-1 and the protein domain structure of RUND-1. (A) The gene structure of rund-1. Exons are depicted as black boxes and introns as black lines. The deletion allele tm3622 is indicated by a red line. (B) RUND-1 contains two Nterminal located coiled-coil (CC)-domains and a C-terminal RUN-domain, predicted by the SMART program (http://smart.embl-heidelberg.de/). aa, amino acid; bp, base pairs. 
The protein RUND-1 consists of 549 amino acids and contains two N-terminal

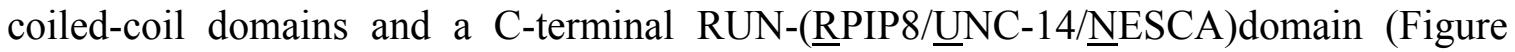
VII.5B).

Similar to unc-108/rab-2 mutants, rund-1 mutants exhibit movement defects, but to a lesser extent (M. Ailion, unpublished data). The overall speed of rund-1(tm3622) (15.20 \pm 1.52$)$ mutants was reduced by about $30 \%$ of wild type worms $(21.92 \pm 1.21)$ (Figure VII.6). Furthermore, rund-1 mutants showed little spontaneous movement on food (own observation, M. Ailion, unpublished data). Interestingly, these mutants displayed coordinated movement as a respond to stimuli like touches, which indicates that RUND-1 plays a role in regulating movement, rather than in executing it (own observation, M. Ailion, unpublished data).

Movement Assay

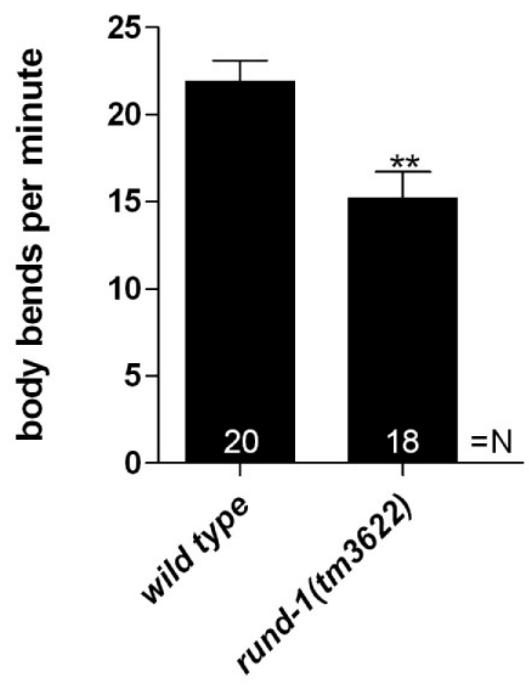

Figure VII.6. rund-1 mutants display movement defects. Young adult worms of each strain were transferred to non-seeded plates, adjusted for several minutes before the number of body bends per min of each worm was recorded. rund-1(tm3622) mutants displayed a lower rate of locomotion compared to wild type animals. Error bars $=$ s.e.m. ( ${ }^{* *}, \mathrm{P}<0.001$; Student's t-test). 
In order to test whether rund-1 mutants exhibit a slow movement phenotype due to defects in DCV trafficking, we subjected these mutants to the NLP-21-VENUS assay (Sieburth et al, 2007). Like unc-108/rab-2 mutants, a decreased NLP-21-derived VENUS fluorescence was observed in the DNC in rund-1(tm3622) mutants. Inactivation of RUND-1 led to a $77.99 \pm 2.42 \%$ decrease of VENUS fluorescence in the axons of the

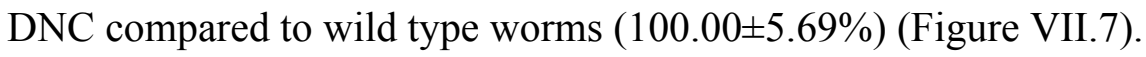

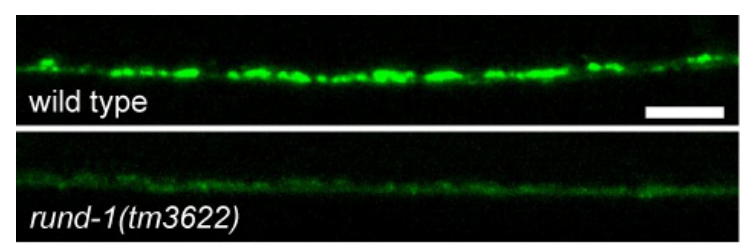

NLP-21-VENUS

in dorsal nerve cord

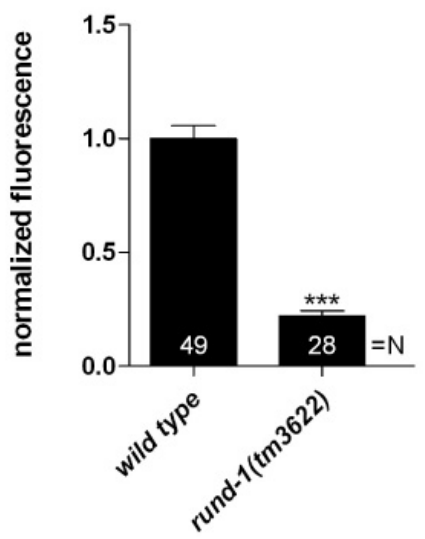

Figure VII.7. RUND-1 is involved in DCV trafficking. rund-1(tm3622) mutants display decreased NLP-21-derived VENUS fluorescence in the DNC by $77.99 \pm 2.42 \%$. Scale bar represent $5 \mu \mathrm{m}$. Error bars $=$ s.e.m. $(* * *, \mathrm{P}<0.0001$; Student's t-test).

These findings suggest that RUND-1 plays a role in DCV trafficking. Since unc108/rab-2 mutants showed comparable defects to rund-1 mutants, it was likely that both proteins act in the same pathway. The analysis of the locomotion and DCV maturation defects of unc-108, rund-1 double mutants revealed that indeed RUND-1 and RAB-2 are in the same genetic pathway (M. Ailion, unpublished data). 


\section{VII.3 RUND-1 interacts with RAB-2 in a yeast two-hybrid analysis}

Since RUND-1 and RAB-2 act in the same pathway, we attempted to test whether there might be also a direct interaction of active GTP-bound RAB-2 and RUND-1 using Y2H analysis. We could show that RUND-1 bound to RAB-2 in a GTP-dependent manner (Figure VII.8A). To determine whether the interaction between RUND-1 and RAB-2 is specific, we screened all C. elegans RAB proteins against RUND-1 in $\mathrm{Y} 2 \mathrm{H}$ (Figure VII.8B). We found that only RAB-2 in its constitutively GTP-bound form interacted with RUND-1. This result suggests that the interaction between RAB-2 and RUND-1 is highly specific and that RUND-1 is a novel RAB-2 effector.
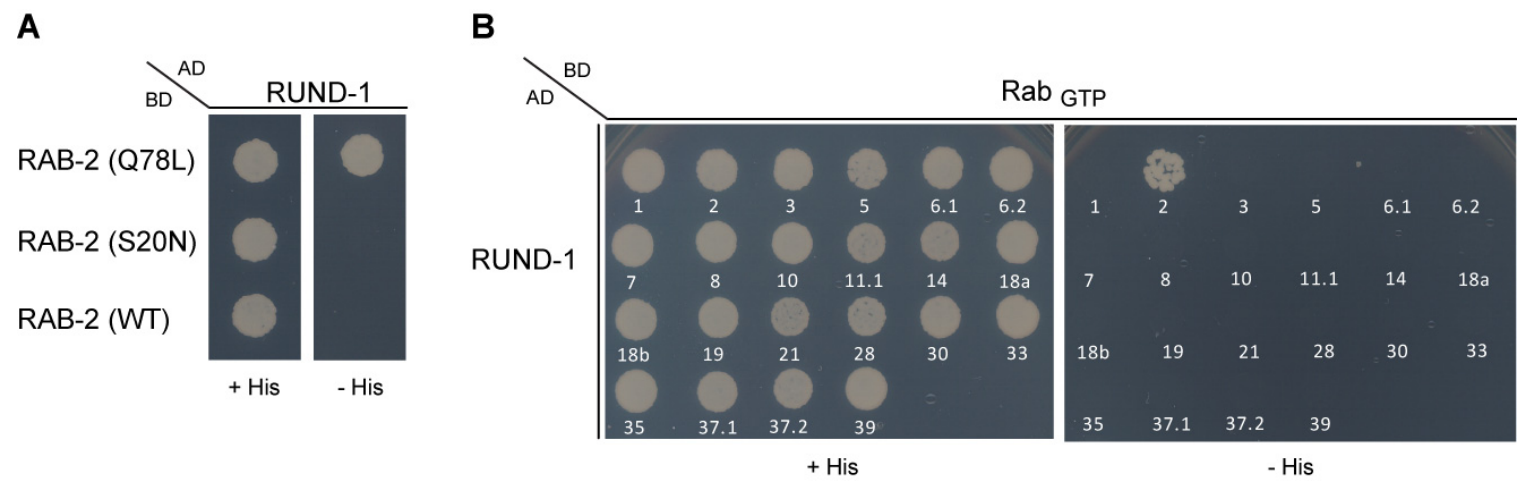

Figure VII.8. RUND-1 specifically interacts with constitutively active, GTP-bound RAB-2 in Y2H. (A) Interaction between RUND-1 and RAB-2 is GTP-dependent suggesting that RUND-1 is an effector of RAB-2. (B) All C. elegans RAB proteins in their constitutively GTP-bound form were tested against RUND-1. RUND-1 specifically interacted with RAB-2 (Q78L). AD, Gal4p DNA activation domain fusion; BD, Gal4p DNA binding domain fusion; His, histidine; $\mathrm{RAB}_{\mathrm{GTP}}$, constitutively GTP-bound RAB GTPase; WT, wild type.

We, then, determined the domain of RUND-1, which is required for the interaction with the small GTPase RAB-2. For this, we constructed different fragments of RUND-1 based on the domain structure of RUND-1 and tested for interaction in $\mathrm{Y} 2 \mathrm{H}$ analysis (Figure VII.9). A fragment containing only the two N-terminal coiled-coil (CC) domains did not interact with GTP-bound RAB-2. In contrast, for a RUND-1 fragment containing the RUN-domain (262-549 aa) we observed an interaction with GTP-bound RAB-2. Attempts to further shorten this fragment resulted in loss of interaction with RAB-2 (data not shown). Interestingly, it was previously shown that an upstream $\alpha$-helix 
is important for proper folding of RUN-domains (Kukimoto-Niino et al, 2006). This upstream $\alpha$-helix was missing in our shorter RUN-domain fragments, which might have resulted in the loss of interaction. These findings indicate that RUND-1 interacts via its RUN-domain with RAB-2.
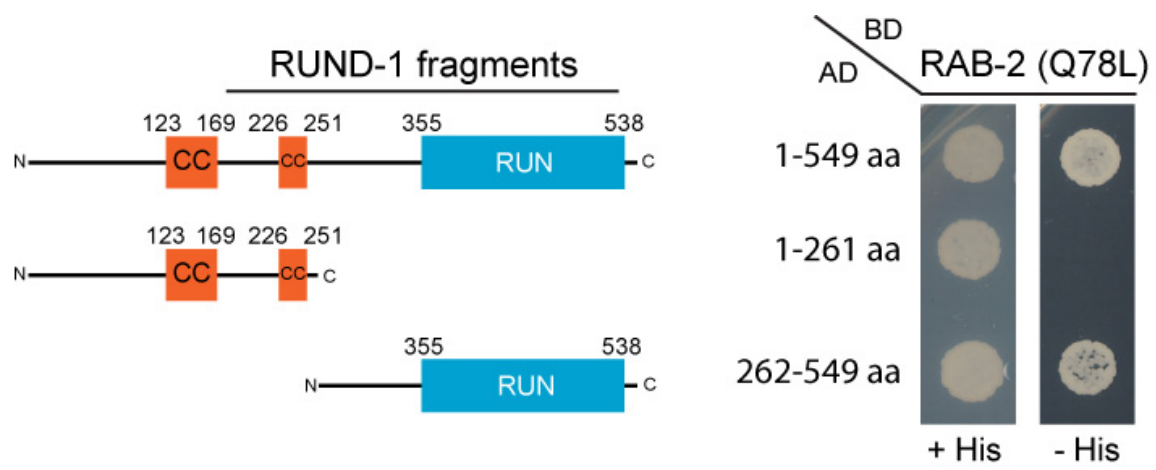

Figure VII.9. RUND-1 might interact with RAB-2 via its RUN-domain. Different fragments of RUND-1 were tested in $\mathrm{Y} 2 \mathrm{H}$ for interaction with constitutively GTP-bound form of RAB-2 (Q78L). A RUND-1 fragment containing both coiled-coil (CC)-domains did not interact with RAB-2 (Q78L), whereas a fragment harboring the RUN domain (262-549 aa) bound to RAB-2 (Q78L). AD, Gal4p DNA activation domain fusion; BD, Gal4p DNA binding domain fusion; His, histidine.

Previously, it has been shown that other RUN-domains served for interaction with small GTPases of the Rab and Rap families (Yang et al, 2007). Therefore, we tested possible interactions between RUND-1 and the C. elegans RAP proteins, as well as RAS1 and RAL-1 (Figure VII.10). However, no interaction between RUND-1 and these tested small GTPases in their wild type state as well as in their predicted constitutively GTPbound state (Chen et al, 2011; Frische et al, 2007; Lundquist, 2006) were observed. Thus, the RUN-domain of RUND-1 seems to specifically interact with active GTP-bound RAB2. 

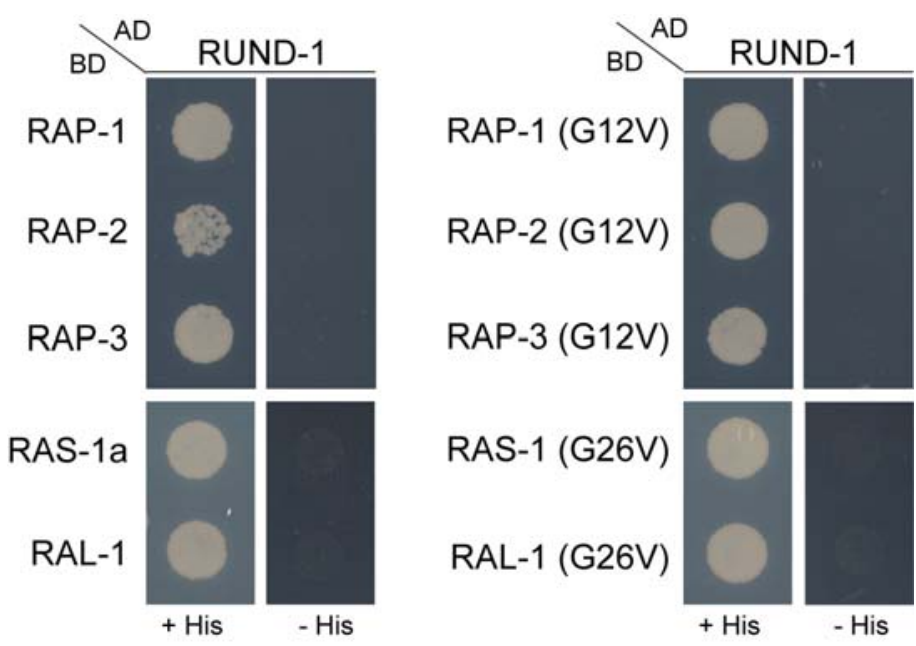

Figure VII.10. RUND-1 does not interact with other small GTPases in a Y2H analysis. Since RUN-domains were shown to contribute to interaction with Rap GTPases, we tested interaction with C. elegans RAP proteins, as well as with RAS-1a and RAL-1 in their wild type and their predicted constitutively GTP-bound state (GV mutation) (Chen et al, 2011; Frische et al, 2007; Lundquist, 2006). However, no interaction between tested proteins was detected in Y2H. AD, Gal4p DNA activation domain fusion; BD, Ga14p DNA binding domain fusion; His, histidine.

\section{VII.4. RUND-1 interacts with the putative RAB-2 GAP, TBC-8}

In section VII.1, we could show that the putative RAB-2 GAP, TBC-8, interacted with the RAB-2 effector, RIC-19, which was surprising. To determine whether RUND-1 also interacts with TBC-8, we tested possible interaction using $\mathrm{Y} 2 \mathrm{H}$ and coIP experiments in HEK293 cells (Figure VII.11). We detected interactions between TBC-8 and RUND-1 in both, Y2H (Figure VII.11A) and co-IP analysis (Figure VII.11B), whereas this binding seemed to be less strong than the interaction between TBC-8 and RIC-19. More V5-TBC-8 could be pulled down with GFP-RIC-19 (Figure VII.3C). 
A

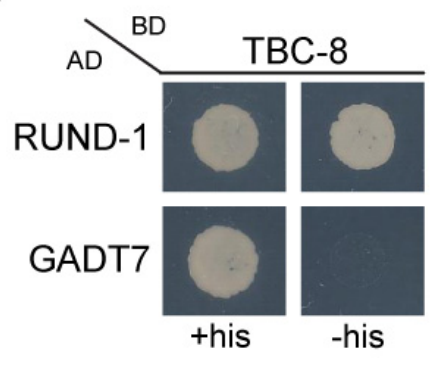

B

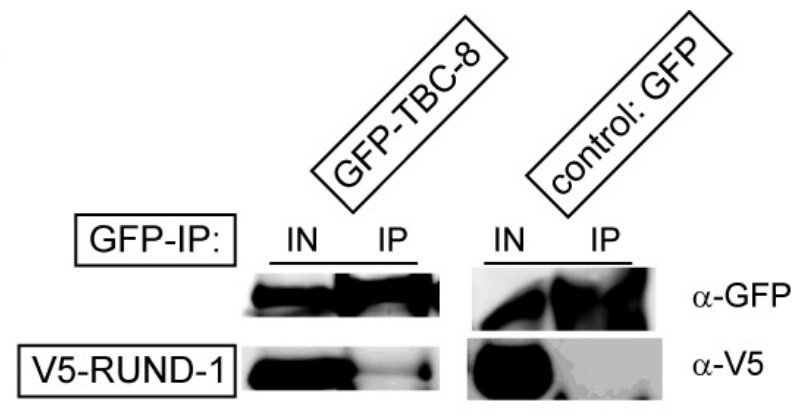

Figure VII.11. RUND-1 interacts with the putative RAB-2 GAP, TBC-8. (A) Interaction could be detected between RUND-1 and TBC-8 in Y2H. The empty vector pGADT7 was used for testing self-activation of TBC-8. (B) A co-IP experiment confirmed interaction between TBC8 and RUND-1. HEK293 cells were co-transfected with constructs expressing GFP-tagged TBC8 (or GFP alone as control) and V5-RUND-1. An anti-GFP antibody was used to precipitate GFPTBC-8 or GFP as control (GFP-IP). Interactions were visualized on Western blots. AD, Gal4p DNA activation domain fusion; $\mathrm{BD}$, Gal4p DNA binding domain fusion; His, histidine; IN, Input; IP, immunoprecipitation.

In order to study which domains of TBC-8 and RUND-1 contribute to the interaction, we performed a domain mapping analysis of these proteins using the $\mathrm{Y} 2 \mathrm{H}$ system. We observed that the RUN-domain of TBC-8 is required for interaction with RUND-1 (Figure VII.12), the same fragment, which was required for the interaction with RIC-19 (Figure VII.4). In contrast, the TBC-domain did not seem to be important for binding to RUND-1 (Figure VII.12A). The involvement of the RUN-domain of TBC-8 in the interaction with RUND-1 could be confirmed by co-IP experiments, however, the observed interaction was again rather of low affinity (Figure VII.12B). 
A

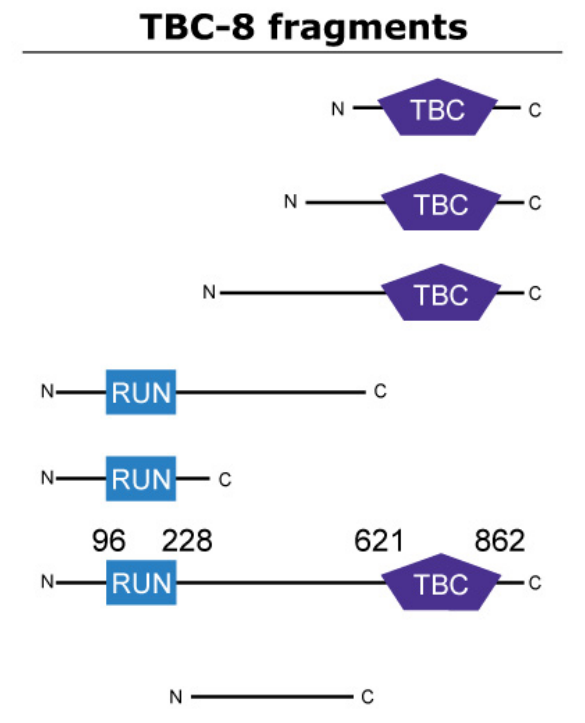

B

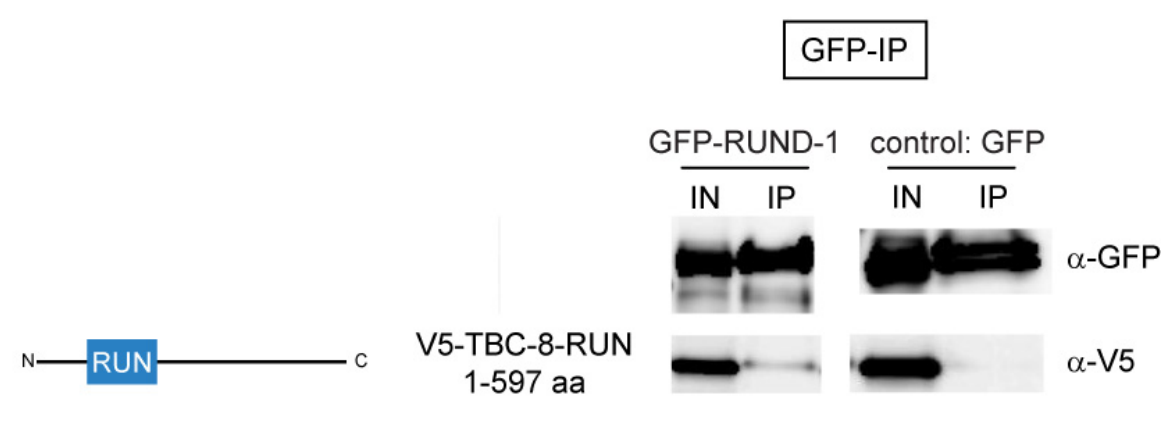

yeast two-hybrid

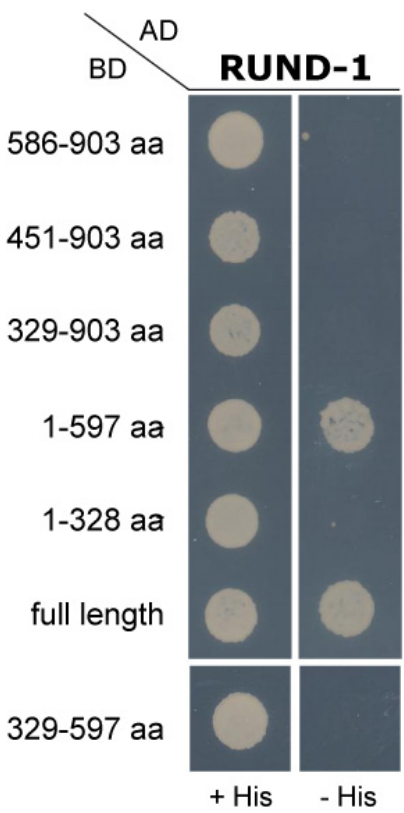

co-immunoprecipitation

Figure VII.12. Domain mapping of TBC-8 for interaction with RUND-1. (A) Full-length TBC-8 as well as a TBC-8 construct harboring the RUN-domain (1-597 aa) interacted with RUND-1 in a Y2H analysis, whereas no interaction was detected between RUND-1 and TBC-8 fragments harboring the TBC-domain. Attempts to shorten the TBC-8 RUN-domain resulted in a loss of the interaction. (B) A co-IP experiment confirmed interaction between the RUN-domain of TBC-8 and RUND-1. HEK293 cells were co-transfected with constructs expressing GFPtagged RUND-1 (or GFP alone as control) and V5-TBC-8-RUN (1-597 aa). An anti-GFP antibody was used to precipitate GFP-RUND-1 or GFP as control (GFP-IP). Interactions were visualized on Western blots. AD, Gal4p DNA activation domain fusion; $\mathrm{BD}$, Gal4p DNA binding domain fusion; His, histidine; IN, Input; IP, immunoprecipitation. 


\section{VII.5 RUND-1 interacts with the RAB-2 effector, RIC-19}

Since RUND-1 interacts with TBC-8 and RAB-2, and TBC- 8 binds to RIC-19, we wondered whether RUND-1 and RIC-19 might also bind to each other. Unfortunately, we could not test interaction of RUND-1 with RIC-19 in the Y2H system, because both proteins displayed strong self-activation when fused to the GAL4 DNA binding domain within the $\mathrm{Y} 2 \mathrm{H}$ system (data not shown). Therefore, we utilized the co-IP experiment to detect possible interaction of RUND-1 with RIC-19. GFP-tagged RIC-19 was able to pull-down V5-RUND-1 from transfected HEK293 cells (Figure VII.13). However, this interaction seemed also to be of low affinity.

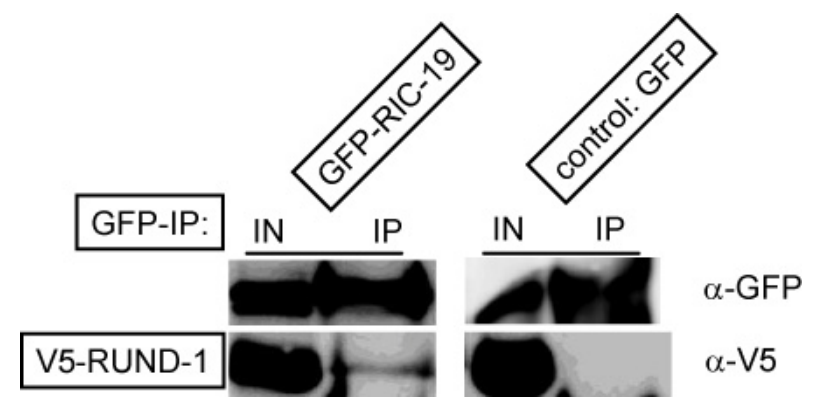

Figure VII.13. RUND-1 interacts with the RAB-2 effector, RIC-19 in a co-IP experiment. HEK293 cells were co-transfected with constructs expressing GFP-RIC-19 (GFP alone as control) and V5-RUND-1. Anti-GFP antibodies were used to precipitate GFP-RIC-19 or GFP alone as control (GFP-IP). Western blots were performed to detect protein interaction. Note: this experiment and the co-IP shown in Figure VII.11B were performed at the same time, therefore, the same GFP/V5-RUND-1-transfected cell-lysate served as the same control. IN, Input; IP, immunoprecipitation.

All these findings indicate that the studied proteins involved in DCV trafficking are able to interact with each other (Figure VII.14). It is thus likely that these proteins are forming a functional complex that is controlled by the activity of RAB-2. The question arises whether they also interact in vivo during DCV trafficking. Therefore, further studies have to be performed to study protein interactions in C. elegans. 


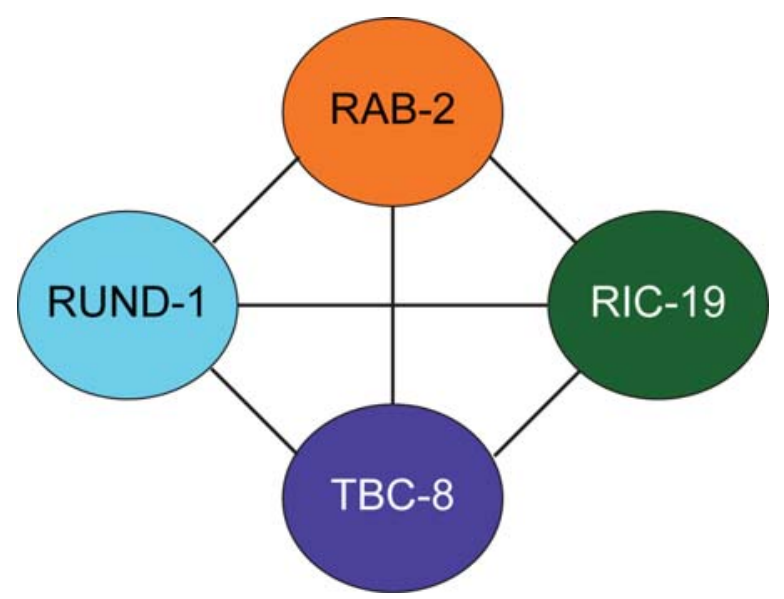

Figure VII.14. Schematic representation of detected interactions of proteins involved in DCV trafficking. We could show that the small GTPase, RAB-2, can interact with the two effectors, RUND-1 and RIC-19 (Sumakovic et al, 2009) and with the Rab GAP, TBC-8. TBC-8 in turn is able to bind to both RAB-2 effectors, RUND-1 and RIC-19. In addition, both effectors interact with each other.

To get a deeper insight into this process, we attempted to study possible genetic interactions between TBC-8, RIC-19 and RUND-1. Therefore, we constructed double mutants of these genes and subjected them to the NLP-21-VENUS assay. This assay revealed that TBC-8 and RIC-19 act in the same pathway (section VII.1), as well as TBC8 and RUND-1 (Figure VII.15). Interestingly, both RAB-2 effectors, RIC-19 and RUND1 showed additive defects in NLP-21-derived VENUS trafficking to the axons, when codepleted (Figure VII.15). These findings indicate that RIC-19 and RUND-1 are involved in parallel branches of the RAB-2 dependent DCV maturation pathway, whereby TBC-8 seems to operate bi-functionally in both pathways. 


\section{NLP-21-VENUS in dorsal nerve cord}

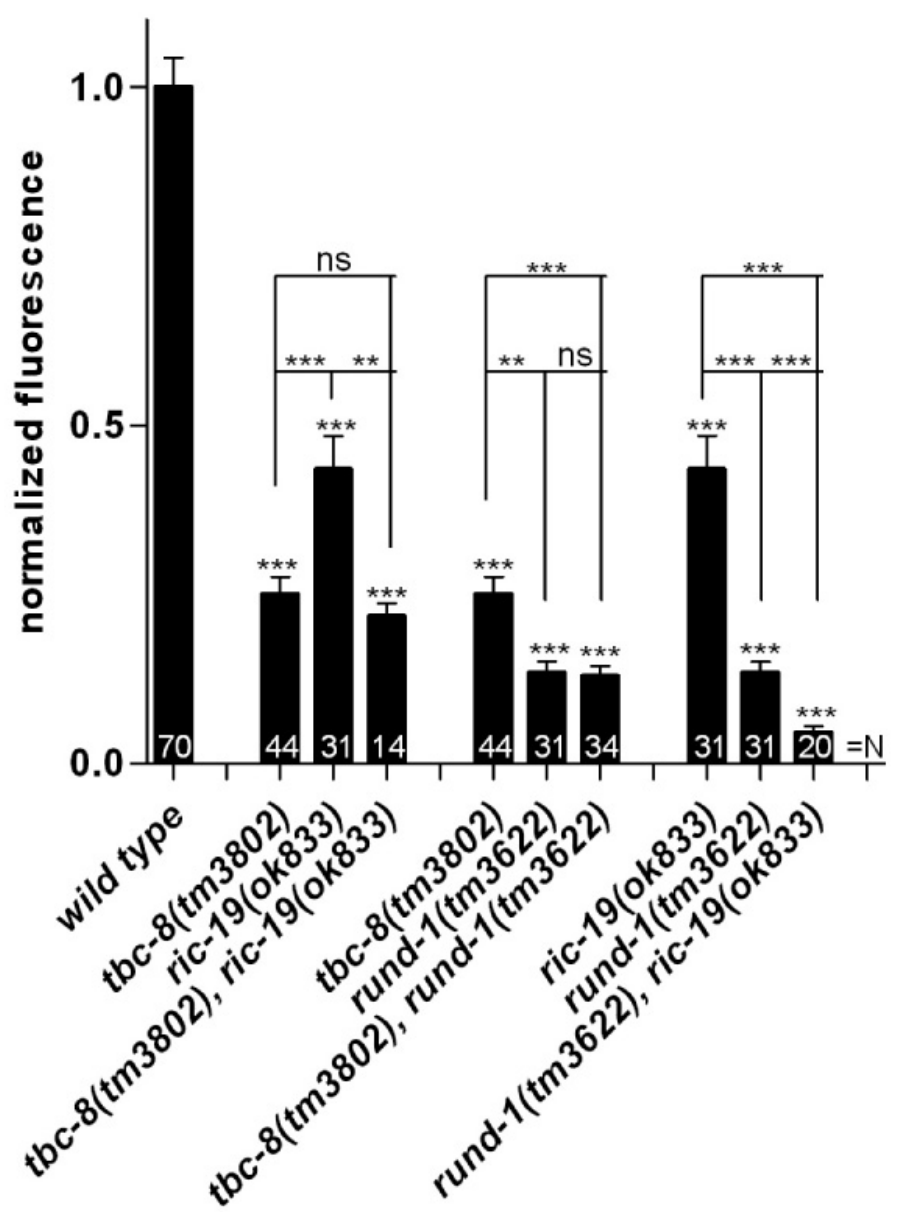

Figure VII.15. Double mutant analysis of TBC-8 and the RAB-2 effectors, RIC-19 and RUND-1 using the NLP-21-VENUS DCV assay. NLP-21-VENUS analysis of the single mutants ric-19(ok833), tbc-8(tm3802) and the double mutant of both in the DNC revealed that RIC-19 and TBC- 8 act in the same genetic pathway. Furthermore, TBC- 8 genetically interacts with RUND-1, since double mutants of both genes followed the phenotype of rund-1(tm3622) single mutants. Interestingly, both RAB-2 effectors, RIC-19 and RUND-1, function in different pathways within DCV maturation, because double mutants of rund-1(tm3622); ric-19(ok833) show additive effects in the NLP-21-VENUS analysis. Error bars $=$ S.E.M. $(* * *, P<0.0001$; Student's t-test). 


\section{VII.6 CCCP-1 is likely to be another RAB-2 effector important for DCV trafficking}

Another factor, which was identified in the screen for unmotivated mutants performed by Michael Ailion, was CCCP-1 (conserved coiled-coil protein). Mutant alleles of ссср-1 gene also showed defects in movement and DCV trafficking like rund-1 and unc-108/rab-2 mutants (M. Ailion, unpublished data).

Like RUND-1, CCCP-1 is also an evolutionarily conserved protein found in Drosophila melanogaster as well in humans (M. Ailion, unpublished data). CCCP-1 possesses three coiled-coil domains distributed along the protein (Figure VII.16).

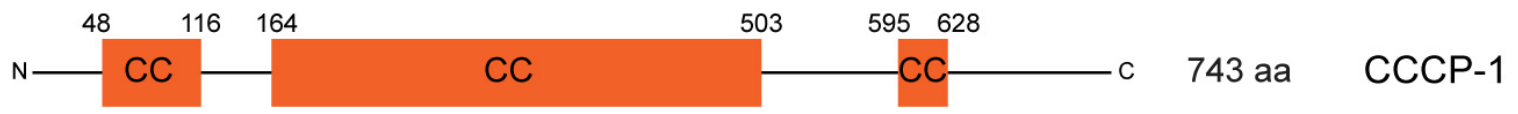

Figure VII.16. Schematic representation of protein domains detected in CCCP-1. The protein CCCP-1 possesses three coiled-coil (CC)-domains of different lengths predicted by the SMART program (http://smart.embl-heidelberg.de/).

In order to test whether CCCP-1 might also be an effector of RAB-2, we performed interaction studies using a $\mathrm{Y} 2 \mathrm{H}$ assay. CCCP-1 interacted with both wild type RAB-2 and constitutively GTP-bound RAB-2 (Q78L) in a Y2H analysis (Figure VII.17A). Unlike many Rab effectors, which only display $\mathrm{Y} 2 \mathrm{H}$ interactions towards the constitutively GTP-bound state of the Rab, CCCP-1 also bound to the wild type RAB-2. This suggests a strong binding between CCCP-1 and RAB-2 considering that only a subpopulation of wild type RAB-2 molecules exists in the GTP bound state. Interestingly, we did not observe an interaction between wild type RAB-2 and the effector RUND-1 in a $\mathrm{Y} 2 \mathrm{H}$ analysis, which would argue for a binding of low affinity (Figure VII.8A). Furthermore, we tested if other RAB GTPases bind to CCCP-1 in a Y2H assay. Figure VII.17 depicts the highly specific interaction between CCCP-1 and RAB-2, since other RAB GTPases in their constitutively, active state did not bind to CCCP-1 in Y2H. 
A

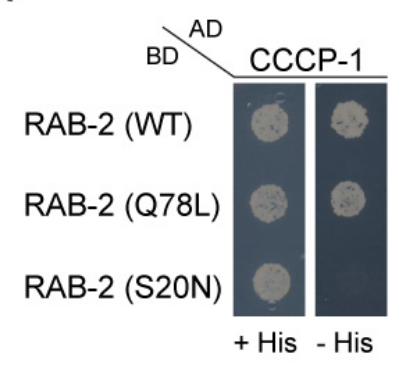

B

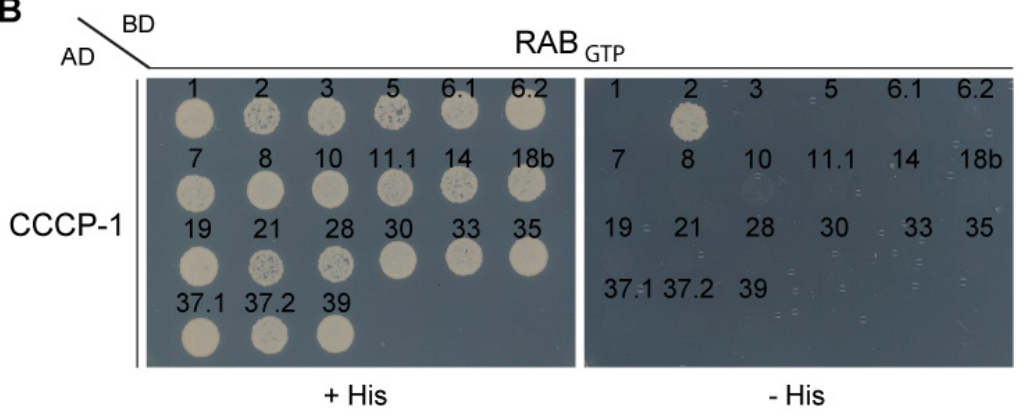

Figure VII.17. CCCP-1 interacts with RAB-2 in a GTP-dependent manner. (A) CCCP-1 interacts with wild type RAB-2 and constitutively GTP-bound RAB-2 (Q78L) in Y2H indicating a strong binding between CCCP-1 and RAB-2. (B) Interaction between CCCP-1 and RAB-2 is highly specific, since other RAB GTPases in their constitutively, active state did not bind to CCCP-1 in Y2H. AD, Gal4p DNA activation domain fusion; BD, Gal4p DNA binding domain fusion; His, histidine.

In order to determine whether CCCP-1 also interacts with the other players of DCV trafficking, we performed a $\mathrm{Y} 2 \mathrm{H}$ analysis among all RAB-2 interaction partners. Interestingly, CCCP-1 did not bind to TBC-8 or the other RAB-2 effectors, RIC-19 and RUND-1 in Y2H (Figure VII.18). This suggests that CCCP-1 is recruited separately to the RAB-2/RIC-19/RUND-1/TBC-8 complex during DCV trafficking. To support this idea, respective double mutants analysis of defects in movement and in the NLP-21VENUS assay would allow deeper insights into CCCP-1 function.

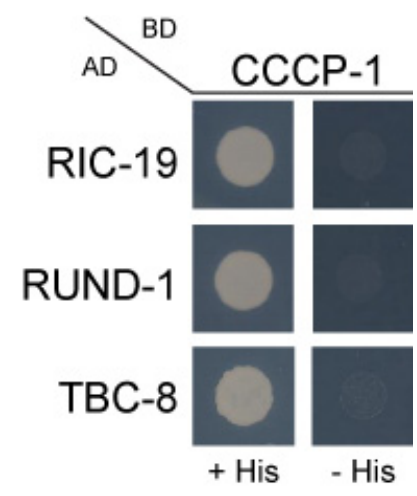

Figure VII.18. CCCP-1 does not interact with TBC-8 nor to the RAB-2 effectors, RIC-19 and RUND-1 in a Y2H analysis. AD, Gal4p DNA activation domain fusion; BD, Gal4p DNA binding domain fusion; His, histidine. 


\section{Chapter 2}

\section{Discussion}

\section{VIII.1 RUND-1 and CCCP-1 are novel effectors of the small GTPase RAB-2 for DCV maturation in C. elegans.}

In this study, we presented two novel factors involved in DCV maturation, RUND-1 and CCCP-1. These proteins were initially identified by a suppressor screen for mutants defective in DCV function in C. elegans (M. Ailion, unpublished data). rund-1 and ссср-1 mutant alleles exhibited similar defects in locomotion and DCV maturation like unc-108/rab-2 mutants (own observation, M. Ailion, unpublished data). At the molecular level, all three mutants showed defects in the sorting of soluble and transmembrane DCV cargo to the axons of the dorsal nerve cord, which is thought to cause the observed uncoordinated locomotion (M. Ailion, unpublished data) (Edwards et al, 2009; Sumakovic et al, 2009). Genetic interaction analysis revealed that rund-1 and cccp-1 act together with $r a b-2$ in the same pathway (M. Ailion, unpublished data). There is a direct interaction between the novel factors and RAB-2, which occurs in a GTPdependent manner. All these findings indicate that RUND-1 and CCCP-1 are novel effectors of the small GTPase RAB-2 functioning during DCV maturation along with RAB-2. Consistent with this hypothesis, RUND-1 and CCCP-1 co-localize with RAB-2 to the trans-Golgi network (TGN) (M. Ailion, unpublished data). However, the 
localization of both factors to the TGN is RAB-2-independent (M. Ailion, unpublished data). This suggests that RUND-1 and CCCP-1 are non-classical RAB-2 effectors.

The localization of classical RAB-2 effectors, such as RIC-19/ICA69, depends on the nucleotide-binding state of RAB-2 (Sumakovic et al, 2009). It has been shown that RIC-19 is recruited to RAB-2 positive compartments in a GTP-dependent manner, whereas RIC-19 is mainly localized in the cytosol when RAB-2 is in its inactive, GDPbound state (Sumakovic et al, 2009). This places RIC-19 together with other classical effectors that are recruited to their sites of action by binding to activated, GTP-bound Rab proteins (Stenmark, 2009). Noteworthy to mention is the existence of other non-classical Rab effectors, which affect the localization of their cognate Rab. An example of such an unconventional effector is the protein EHBP-1, which is an effector of RAB-10 (Shi et al, 2010). It has been shown that EHBP-1 acts upstream of RAB-10 by controlling RAB10's localization to the endosomal compartments in C. elegans (Shi et al, 2010). However, a similar scenario can be excluded for RAB-2 and the effector proteins RUND1 and CCCP-1, since RAB-2 was localized to the Golgi complex independently of its effectors (M. Ailion, unpublished data). Moreover, the localization of RUND-1 and CCCP-1 was also not dependent on each other (M. Ailion, unpublished data). Domain mapping of RUND-1 revealed that the RUN-domain was responsible for the localization of RUND-1 to Golgi membranes, whereas a RUND-1 fragment containing only the coiled-coil domains was mis-localized to the cytosol (M. Ailion, unpublished data). These findings indicate that RUND-1 and CCCP-1 are non-classical effectors of RAB-2.

\section{VIII.2 The function of different protein domains during DCV maturation}

In this study we identified novel factors involved in DCV trafficking, called TBC8, RUND-1 and CCCP-1. Former studies showed that the RAB-2 effector, RIC-19, also plays a role in this process (Edwards et al, 2009; Sumakovic et al, 2009). These four factors possess essential protein domains, such as a TBC-domain, RUN-domains, a BARdomain and coiled-coil (CC) domains (Figure VIII.1). 
TBC-8

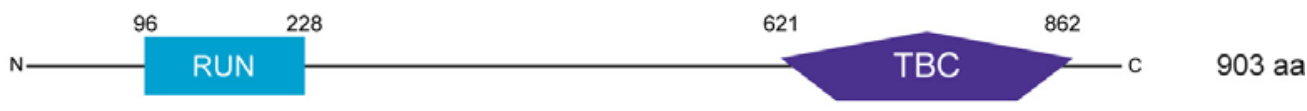

CCCP-1

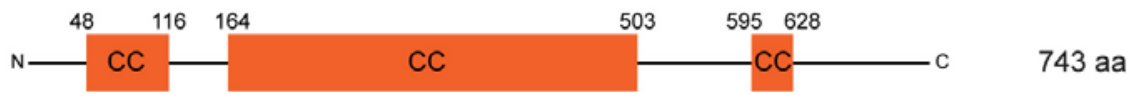

RUND-1

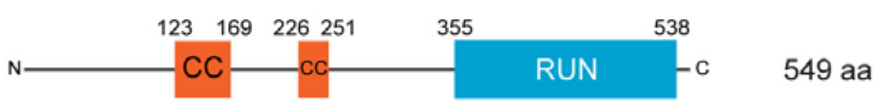

RIC-19

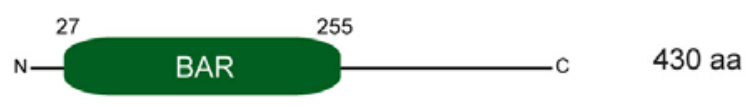

100 aa

Figure VIII.1. Schematic representation of protein domains found in DCV factors, TBC-8, CCCP-1, RUND-1 and RIC-19. Domain sizes are indicated. Protein domains were predicted by the SMART program (http://smart.embl-heidelberg.de/). CC, coiled-coil; TBC, Tre-

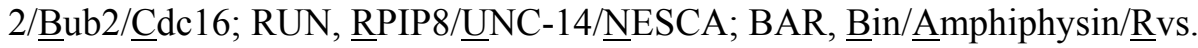

It is interesting to mention that two domains are mainly present in proteins of the RAB-2 effector complex, the RUN-domain and CC-domains. What might be the functions of these protein domains during DCV trafficking?

The crystal structures of two isolated RUN-domain containing proteins (referred to as RUN-domain proteins hereafter) were solved until now, which revealed that RUNdomains form a single globular fold consisting of $\alpha$-helices (Fernandes et al, 2009; Kukimoto-Niino et al, 2006; Recacha et al, 2009). In general, the exact cellular roles of RUN domains are not known yet, but they seem to have diverse and important roles in various cellular signaling processes (Yoshida et al, 2011). Originally, RUN domain proteins were predicted to function in Ras-like GTPase signaling, since it was shown that they act as specific Rab and Rap effectors (Callebaut et al, 2001; Janoueix-Lerosey et al, 1995; Janoueix-Lerosey et al, 1998). Consistent with this prediction, both RUN-domain proteins, RUND-1 and TBC-8, were also able to interact with Rab proteins: RUND-1 specifically bound to RAB-2 and TBC-8 interacted with RAB-2 and RAB-19 in a Y2H assay. In contrast, other RUN domain proteins could not be linked to GTPase function until now, such as UNC-14 and NESCA (Fukuda et al, 2011; MacDonald et al, 2004; 
Ogura et al, 1997). Furthermore, it has been shown that RUN domain proteins interact with motor proteins of actin-filaments or microtubules (Lee et al, 2004; Yoshida et al, 2011). The RUN-domain of Rabip4 was shown to be required for the localization of Rabip4 to endosomes, most likely by the association with a filamentous network, that is likely to be linked to the actin cytoskeleton (Mari et al, 2001). The RUN-domain protein, UNC-14, interacts with the kinesin light chain and might mediate kinesin-1-dependent transport (Sakamoto et al, 2005). Interestingly, a fragment of RUND-1 possessing the RUN-domain localized to the Golgi, whereas a fragment harboring the two CC-domains could not (M. Ailion, unpublished data). Therefore, it is tempting to speculate that the RUN-domain of RUND-1 might also associate with cytoskeletal elements leading to the proper localization of the protein in neurons. During our studies we observed that the RUN domains needed adjacent sequences for their interaction with GTPases or other binding partners [this study, (Recacha et al, 2009)]. It is therefore likely that RUN domains might switch conformation upon binding, inducing a higher order in its structure, which is then able to recruit other factors, because a new interaction surface has been formed.

Surprisingly, the C. elegans genome encodes only six RUN-domain proteins (Figure VIII.2) (www.wormbase.org). We have described two of these, TBC-8 and RUND-1, in this study. The other proteins are TBC-18, UNC-14, and the two uncharacterized proteins Y51H1A.2 and C52E12.4 (www.wormbase.org). The human homolog of TBC-18, called SGSM3, has been identified to be a Rab5 GAP (Haas et al, 2005), whereas UNC-14 is important for axonal elongation and guidance of many neurons by functioning as an adaptor protein of kinesin-1 (Lai \& Garriga, 2004; Ogura et al, 1997; Sakamoto et al, 2005). The human homolog of C52E12.4 has been shown to interact with Rab6 and is therefore called Rab6IP1 (Rab6-interacting protein 1). It contains a DENN-domain, a protein domain found in Rab GEFs (Marat et al, 2011). Since TBC- 8 and RUND-1 are involved in DCV maturation, it is tempting to speculate that RUN-domains might have a novel role in C. elegans. They all might interact with Rab proteins serving in DCV signaling, which has to be further studied. 


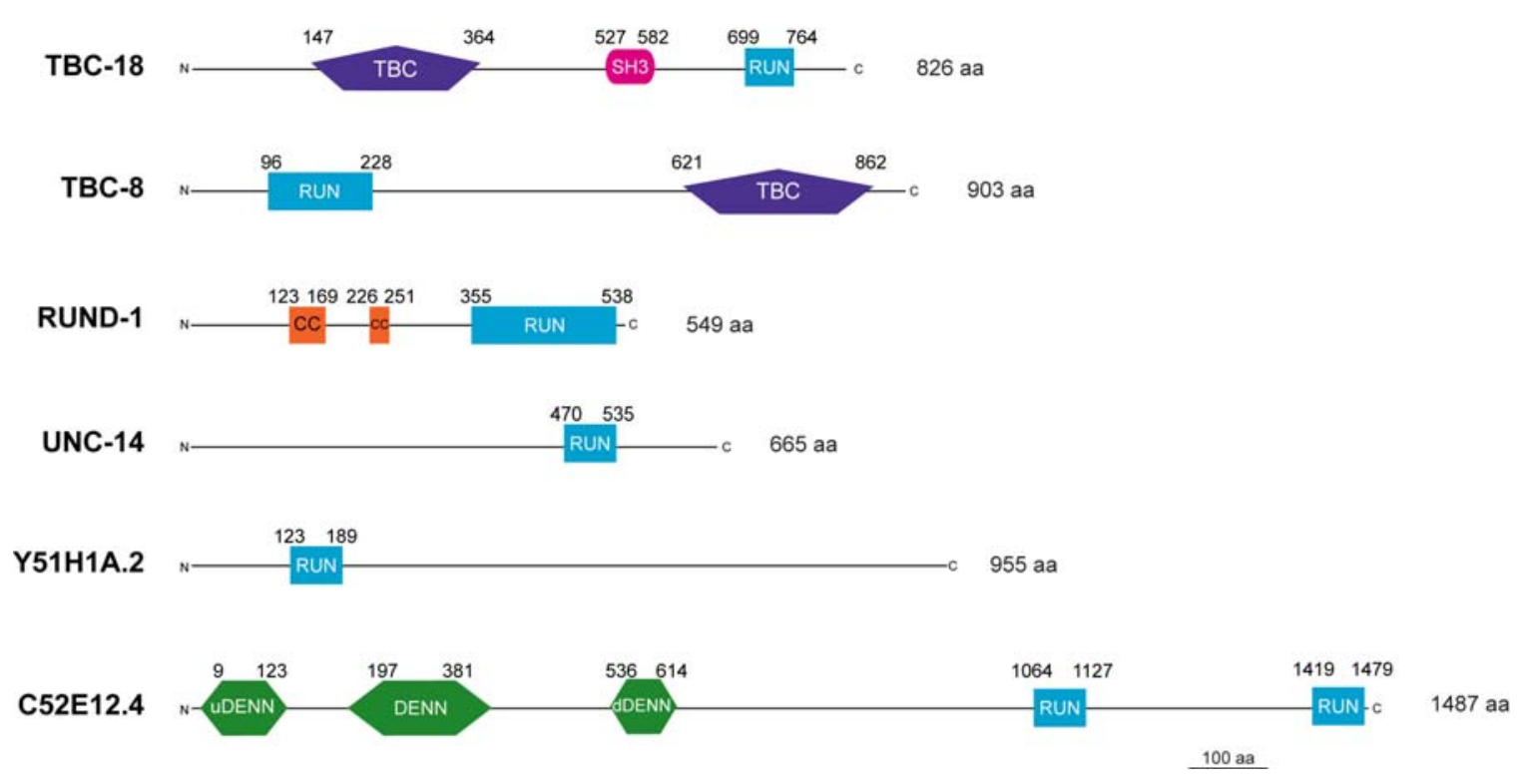

Figure VIII.2. The $C$. elegans genome encodes for six RUN-domain containing proteins. TBC-8 and TBC-18 are potential Rab GAPs, whereas C52E12.4 is likely to be a Rab GEF. RUND-1 was characterized in this study. UNC-14 was shown to be involved in axonal guidance (Ogura et al, 1997). Y51H1A.2 is an uncharacterized protein in C. elegans (www.wormbase.org). Protein domains were predicted by the SMART program (http://smart.embl-heidelberg.de/). CC, coiled-coil; DENN, differentially expressed in normal versus neoplastic cells; uDENN, upstream DENN; dDENN, downstream DENN; SH3, Src homology 3; TBC, Tre-2/ㅁub2/ddc16; RUN, RPIP8/ㄴNC-14/NESCA.

Interestingly, many RUN-domain proteins also possess additional CC-domains, such as RPIP8 (Janoueix-Lerosey et al, 1998), Rabip4 (Cormont et al, 2001; Mari et al, 2001), and RUFY1 (Yang et al, 2002). It is tempting to speculate that CC-domains facilitate the functions of RUN-domains. In general, CC-domains form extended rod like structures that are known to mediate multiple protein-protein interactions (Zhang et al, 2009). It has also been shown that CC-domains of Golgins assist the localization of Rab GTPases to Golgi membranes [reviewed in (Munro, 2011)]. In the case of RAB-2, none of the effectors, RUND-1 or CCCP-1, both possessing CC-domains were required for the localization of RAB-2 to Golgi membranes (M. Ailion, unpublished data). CC-domains consist of two or more $\alpha$-helices, which associate in a supercoiled bundle (Lupas \& Gruber, 2005; Zhang et al, 2009). These domains are able to fold and interact autonomously, independently of their remaining amino acid sequences (Lupas \& Gruber, 2005). Previously, a combined computational-experimental screen demonstrated that 
proteins necessary for vesicular trafficking are enriched in CC-domains mediating interactions (Zhang et al, 2009). For example, SNARE (soluble N-ethylmaleimidesensitive-factor attachment receptor) proteins promote membrane fusion by assembling into coiled-coil tetramers [reviewed in (Jahn \& Scheller, 2006; Ungar \& Hughson, 2003)]. Long coiled-coil proteins, called Golgins that localize to specific Golgi subdomains are important to bridge incoming transport vesicles to their target membrane prior to fusion (Gillingham \& Munro, 2003; Sinka et al, 2008). Other proteins, such as GTPase regulators, motor proteins, cytoskeleton regulators and multi-subunit tethering complexes possess coiled-coil domains important for exhibiting their function in vesicular transport (Zhang et al, 2009).

In summary, RUN-domains and CC-domains are found in many proteins involved in vesicular trafficking and might be important for the correct localization of these proteins. Moreover, they might also mediate interactions with other proteins in order to fulfill their functions within vesicular trafficking.

\section{VIII.3 Regulation of the putative RAB-2 GAP, TBC-8, during DCV maturation}

It is currently unclear how the activity of the putative RAB-2 GAP, TBC-8, is regulated. In general, GAP activity might be controlled in vivo either by their subcellular localization or by additional regulatory mechanism like phosphorylation (Barr \& Lambright, 2010). There are indications that GAP proteins contain regions important for regulating activity through interactions with other proteins (Albert et al, 1999).

In the case of TBC-8, we showed this potential Rab GAP binds to the RAB-2 effector, RIC-19, in $\mathrm{Y} 2 \mathrm{H}$ and co-IP experiments, suggesting that both proteins might form a complex when recruited to RAB-2-positive membranes. This interaction might be important for regulating TBC- 8 activity. On the one hand, TBC- 8 can be inactive when recruited by active RAB-2 via RIC-19 and then activated after recruitment. On the other hand, TBC- 8 can be constitutively active while bound to RIC-19, which then would lead to an immediate deactivation of RAB-2 after recruitment of the this complex. We mapped the binding region of RIC-19 to TBC-8 and showed that RIC-19 binds outside of the 
TBC-domain, to the N-terminal part of TBC-8 containing the RUN domain. Thus, it is currently unlikely that RIC-19 would mask the TBC-domain and inhibit GAP activity. However, the change in TBC-8 localization through RIC-19 might lead to specific interaction with RAB-2, negatively regulating this RAB during DCV maturation in $C$. elegans. Further studies should aim to reveal whether the recruitment of RIC-19 and TBC-8 to RAB-2-positive membranes occurs independently of each other or not. This would give further indications whether RIC-19 possesses regulating activity towards TBC-8.

Interestingly, the interaction between RIC-19 and TBC-8 seems to be conserved because the human homolog of RIC-19, the diabetes autoantigen ICA69, could also bind to TBC-8 in the Y2H system. This indicates that ICA69 might also play an important role in regulating insulin secretion from pancreatic $\beta$-cells since the human homolog of TBC8, SGSM1, is also expressed in the pancreas (Buffa et al, 2008).

TBC-8 activity might also be regulated by its interaction with active GTP-bound RAB-19. However, we did not detect any defects in DCV maturation in rab-19(ok1845) deletion mutant animals. This result suggests that RAB-19 might not be involved in DCV trafficking and that TBC-8 together with RAB-19 might act in another DCV-independent pathway.

\section{VIII.4 The RAB-2 effector complex might be highly dynamic}

The involvement of several Rab GTPases in Rab/GAP/GEF cascades during membrane trafficking has been demonstrated [reviewed in (Hutagalung \& Novick, 2011)]. During Rab GAP cascades, the downstream Rab recruits the Rab GAP for the upstream Rab via its effector complex (Stenmark, 2009). For example in yeast, in the Ypt1p/Ypt32p cascade Ypt1p associates with the Golgi and regulates ER to Golgi trafficking (Sclafani et al, 2010). The GAP for Ypt1, Gyplp, is recruited by interaction with activated Ypt32p, the downstream Rab (Rivera-Molina \& Novick, 2009a). It has been shown that this counter-current GAP cascade limits the overlap between activated Rab GTPases within the yeast secretory pathway (Barrowman et al, 2010; Rivera-Molina 
\& Novick, 2009b). Therefore, a GAP cascade sharpens the boundaries during the Rab conversion cascades supporting sorting of cargo into the downstream Rab domain and thus, enhancing the directionality of the transport process (Hutagalung \& Novick, 2011).

However, the situation might be different in the case of RAB- 2 and TBC- 8 where the active Rab GTPase would recruit its own GAP through its effector complex. This would suggest that RAB-2 might not participate in a traditional Rab conversion cascade. Further support for this view comes from the fact that we were unable to identify a second Rab GTPase in C. elegans showing the same DCV maturation phenotype despite screening all Rab GTPases in C. elegans (our unpublished data). It is therefore likely that RAB-2 participates in DCV maturation through a membrane trafficking mechanism distinct from the previously described Rab cascades. However, since we were unable to test RAB-2 specific GTPase activity of TBC- 8 due to protein insolubility, we cannot completely exclude that RAB-2 might be part of a Rab cascade. Interestingly, as far as we are aware, there is only one example in literature where a Rab effector binds to the respective Rab GAP to enhance deactivation of the Rab (Hokanson \& Bretscher, 2012). Therefore, such a negative feedback loop might represent a novel mechanism to regulate Rab function. We propose that the recruitment of the TBC- 8 GAP might ensure that the effector complex is short-lived and thus, highly dynamic. It is likely that the effector complex just exists to bring several components together important for DCV maturation and then has to be rapidly disassembled to allow another round of assembly.

\section{VIII.5 The role of the RAB-2 effector complex during DCV maturation}

How does the putative RAB-2 GAP, TBC- 8 , regulate RAB-2 activity and how do all the other identified factors contribute to DCV maturation? We have previously shown that RAB-2 dependent cargo retention is achieved by a dynamic ON/OFF cycle of RAB-2 at the Golgi-endosomal interface, since both constitutively active and constitutively inactive rab-2 mutants exhibited the same DCV phenotype (Edwards et al, 2009; Sumakovic et al, 2009). Thus, RAB-2 most likely undergoes several rounds of activation

and deactivation in order to fulfill its task during DCV maturation. The finding that the 
RAB-2 effector, RIC-19, interacts with the putative RAB-2 GAP, TBC-8, supports this view. Thus, once RAB-2 is locally activated at the Golgi-endosomal interface, it might recruit the RIC-19/TBC-8 complex, which in turn will lead to its deactivation and release from its membrane localization. This will also then cause a cytosolic redistribution of RIC-19 and TBC-8. The fact that over-expressed RIC-19 and TBC-8 showed remarkable membrane localization even in the absence of RAB-2 suggests that RIC-19 and TBC-8 might also bind to another factor that is membrane-localized, independent of RAB-2. This factor might be either RUND-1 or CCCP-1, because both proteins localize to the Golgi complex in a RAB-2-independent manner (M. Ailion, unpublished data). The observation that this massive membrane localization of RIC-19 and TBC-8 is only seen by over-expression of both proteins suggests that the interactions might be of low affinity in vivo. Thus, in a normal situation, the effective concentrations would be insufficient for a productive interaction and thus recruitment. In this case, the interaction would require activated RAB-2 for efficient recruitment of RIC-19 and TBC-8 to membranes. This would postulate that active GTP-bound RAB-2 would interact with the RIC-19/TBC-8 complex as well as with this Golgi-localized factor that might be RUND-1 or CCCP-1. Accordingly, an inactivation of RAB-2 by its GAP, TBC-8, would lead to a rapid disassembly of the complex and a release of its components for a new round of recruitment. This low-affinity hypothesis would allow a fast and transient interaction of the required players in order to guarantee a dynamic regulation of RAB-2, which in turn provides the basis for a series of remodeling events at the Golgi-endosomal interface. However, from our current set of data, we cannot exclude the possibility that RIC-19 and TBC- 8 are recruited separately from each other to the Golgi and start to interact at RAB-2 positive vesicles. Furthermore, it is possible that another RAB-2 effector is also able to recruit TBC-8 to the Golgi in the absence of RIC-19 explaining the less severe DCV phenotype of ric-19(ok833) mutants compared to tbc-8(tm3802) deletion mutants.

More efforts have to be made to solve the function of the TBC-8/RIC-19 interaction. One possibility to study the dependence of recruitment of TBC-8 and RIC-19 is to use different mutant background and study recruitment of the other factors. Since we favor the low-affinity hypothesis, it is necessary to use single-copy insertions of transgenes into the genome of C. elegans, since we demonstrated that over-expression of 
effectors led to autonomous membrane recruitment, which would make the recruitment study impossible.

What are the functions of CCCP-1 and RUND-1 during DCV maturation? We could show that these proteins are Golgi-localized independently of RAB-2 (M. Ailion, unpublished data). Since both proteins contain CC-domains, especially CCCP-1 is predicted to form coiled-coils along most of its entire length, it is possible that they have similar functions like the so-called Golgins. Golgins are long, coiled-coil proteins, which are resident in the Golgi membranes [reviewed in (Munro, 2011)]. They label the identity of specific Golgi regions and bind to vesicles through the interaction with various Rab proteins. It is believed that they form a tentacular matrix allowing them to capture vesicles in the vicinity of the Golgi stacks (Munro, 2011). In a similar way, RUND-1 and CCCP-1 could bind to RAB-2-positive dense-core vesicles in order to prevent them from diffusion away from the Golgi during DCV maturation. Accordingly, inactivation of RUND-1 and CCCP-1 would cause loss of these vesicles into the endosomal-lysosomal pathway resulting in less DCV cargo at the axons.

In addition, RUND-1 might be required to preserve structural integrity of the Golgi and generates certain membrane domains on the Golgi that are important for RAB2 functions. These RAB-2-labeled Golgi membranes might ensure rearrangement events induced by RAB-2 effectors, which are required for efficient maturation (Tooze et al, 2001). The recruitment of RIC-19 might help to create or stabilize these membrane domains by its BAR domain, which recognizes curved membranes found on vesicles [reviewed in (Frost et al, 2009; Rao \& Haucke, 2011)]. By recruitment of the negative regulator of RAB-2, TBC-8, a dynamic network is ensured to retain specific cargo in DCVs in order to prevent their mis-sorting to the endosomal-lysosomal pathway. In summary, all these players along with RAB-2 might be specifically required to stabilize membrane domains on DCVs to facilitate sorting and the retention of cargo during DCV maturation (Figure VIII.3). 


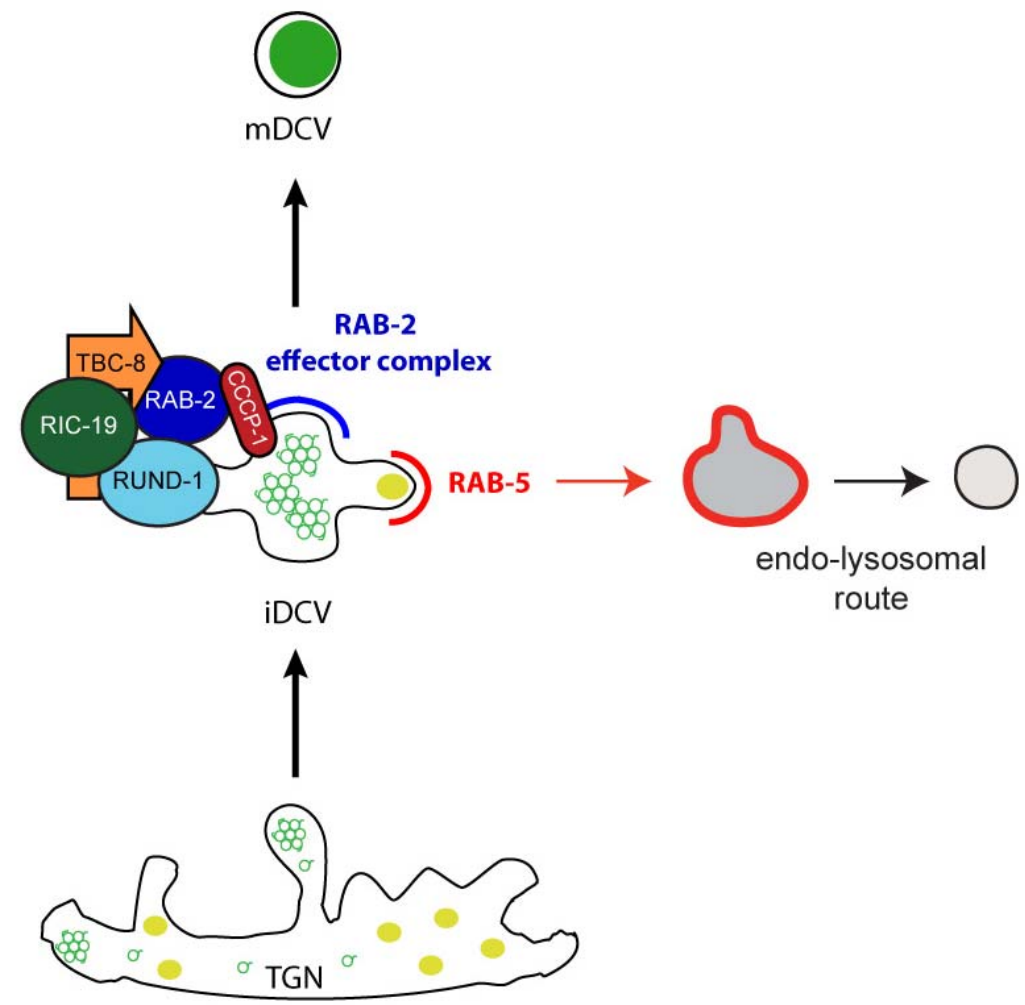

Figure VIII.3. Schematic representation of a novel RAB-2 effector required for DCV maturation in $\boldsymbol{C}$. elegans neurons. The maturing DCV compartment might exhibit different domains on its surface, which are important for specific function ensured by a particular set of proteins (e.g. RAB-2 domains). The recruitment of the RAB-2 effector complex (RIC-19/TBC-8) to RAB-2 positive membranes might be important for dynamic cycling of RAB-2. All these players along with RAB-2 might be specifically required to stabilize membrane domains on DCVs to facilitate sorting and the retention of cargo during DCV maturation. From our previous work, we predict that RAB-5 might form a domain from which mis-sorted cargo is removed to the endo-lysosomal pathway [our unpublished data; (Sumakovic et al, 2009)]. However, the specifics of this schematic representation are highly speculative, because the molecular mechanisms of interactions have not yet been elucidated. Source: picture modified from (Tooze et al, 2001), with permission from Elsevier.

It is conceivable that active RAB-2 would also help to accept retrograde trafficking carriers within the Golgi-endosomal interface to be delivered back to the maturing DCV compartment. This would explain why in rab-2 mutants, cargo from maturing DCVs is lost to the endosomal-lysosomal pathway (Edwards et al, 2009; Sumakovic et al, 2009). Thus, the next chapter of this study elucidates the function of the retrograde transport pathway in DCV maturation. It has to be highlighted again, that 
cargo which is retained within maturing DCVs by most likely binding to a cargo receptor is not affected in rab-2 mutants. Neuropeptides itself, for example that are aggregated within the dense-core are not removed in rab-2 mutants (Sumakovic et al, 2009). In contrast, soluble cargo (represented by soluble VENUS) and some of the transmembrane cargo (represented by the transmembrane protein IDA-1) are lost from maturing DCVs in rab-2 mutants. 


\section{Chapter 3}

\section{Role of the retrograde transport pathway in dense-core vesicle maturation}

\section{Specific Introduction}

\section{IX.1 Role of tethering factors in intracellular transport}

The transport of cargo molecules between organelles of the endomembrane system is organized by vesicular membrane carriers. Their transfer from the donor to the acceptor membrane is characterized by four essential steps: vesicle budding from a donor membrane/compartment, vesicle transport, vesicle tethering and vesicle fusion to a acceptor membrane/compartment (Figure IX.1). Each step requires the coordination of a cohort of proteins, such as small GTPases, coat proteins, motor proteins, soluble Nethylmaleimide-sensitive-factor attachment receptor proteins (SNAREs) and tethering factors to guarantee specificity and efficiency [reviewed in (Cai et al, 2007)]. 


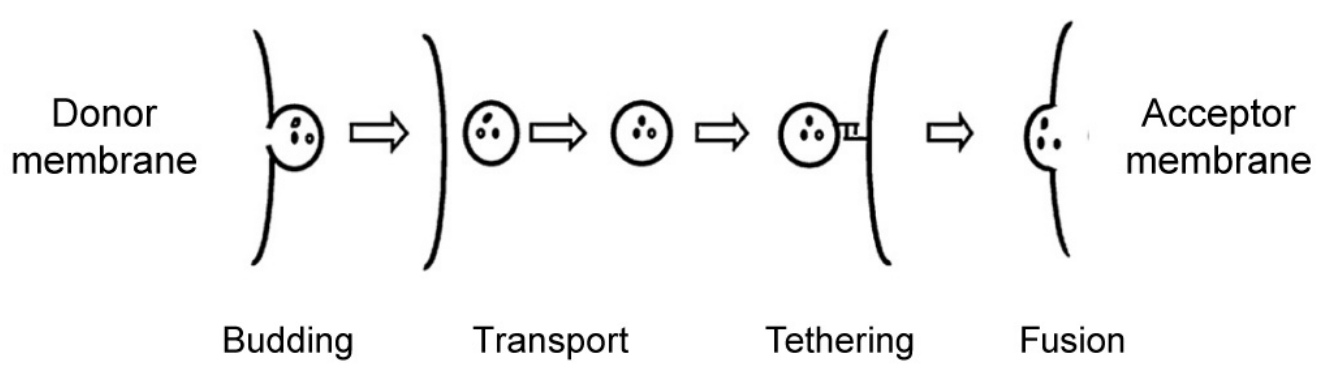

Figure IX.1. Transport of cargo-loaded vesicles between organelles occurs in four distinct steps. Vesicles bud from a donor compartment, are transported to the acceptor compartment, where they are tethered to the membrane and subsequently undergo fusion to release their cargo. Source: reprinted from (Segev, 2011), with permission from Elsevier.

During the last steps, SNARE proteins present on the vesicle and the acceptor compartment assemble into a tight bundle, which brings the two membranes into close proximity leading to fusion of the lipid bilayers (Jahn \& Scheller, 2006; Sudhof \& Rothman, 2009). Small GTPases and tethering factors cooperate with SNARE proteins during the tethering and fusion events (Cai et al, 2007; Stenmark, 2009; Yu \& Hughson, 2010). Tethering factors are large coiled-coil proteins or protein complexes that facilitate long-range interactions between the two distinct membranes, before SNARE proteins are able to interact (Cai et al, 2007; Yu \& Hughson, 2010).

Tethering factors are classified into two groups, homodimeric long coiled-coil proteins and heteromeric multi-subunit tethering complexes (MTCs) based on their protein structure (Figure IX.2) [reviewed in (Barr \& Short, 2003; Bonifacino \& Hierro, 2011; Cai et al, 2007; Gillingham \& Munro, 2003; Yu \& Hughson, 2010)]. Both types of tethering factors are structurally unrelated but share properties and functions, such as being Rab effectors (Yu \& Hughson, 2010). So far, the localization of long coiled-coil proteins is restricted to the Golgi complex (e.g. Golgins) and to endosomes (e.g. EEA1 [early endosome antigen 1]) (Figure IX.2). They are characterized by long stretches of heptad repeats and form extended rod-like structures (Sztul \& Lupashin, 2006). Many of these coiled-coil tethers exist as dimers (Sztul \& Lupashin, 2006). Besides their role in tethering vesicles to the target membrane, a function in maintaining the structural 
integrity of the Golgi matrix is described (Gillingham \& Munro, 2003). Furthermore, they might act as scaffold proteins for the assembly of other factors important for fusion (Gillingham \& Munro, 2003).

\section{IX.2 Multi-subunit tethering complexes}

Subgroups of multi-subunit tethering complexes (MTCs) are represented by TRAPP (transport protein particle), HOPS (homotypic fusion and vacuole protein sorting), CORVET (class C core vacuole/endosome tethering complex) and CATCHR (complex associated with tethering containing helical rods) complexes (Figure IX.2).

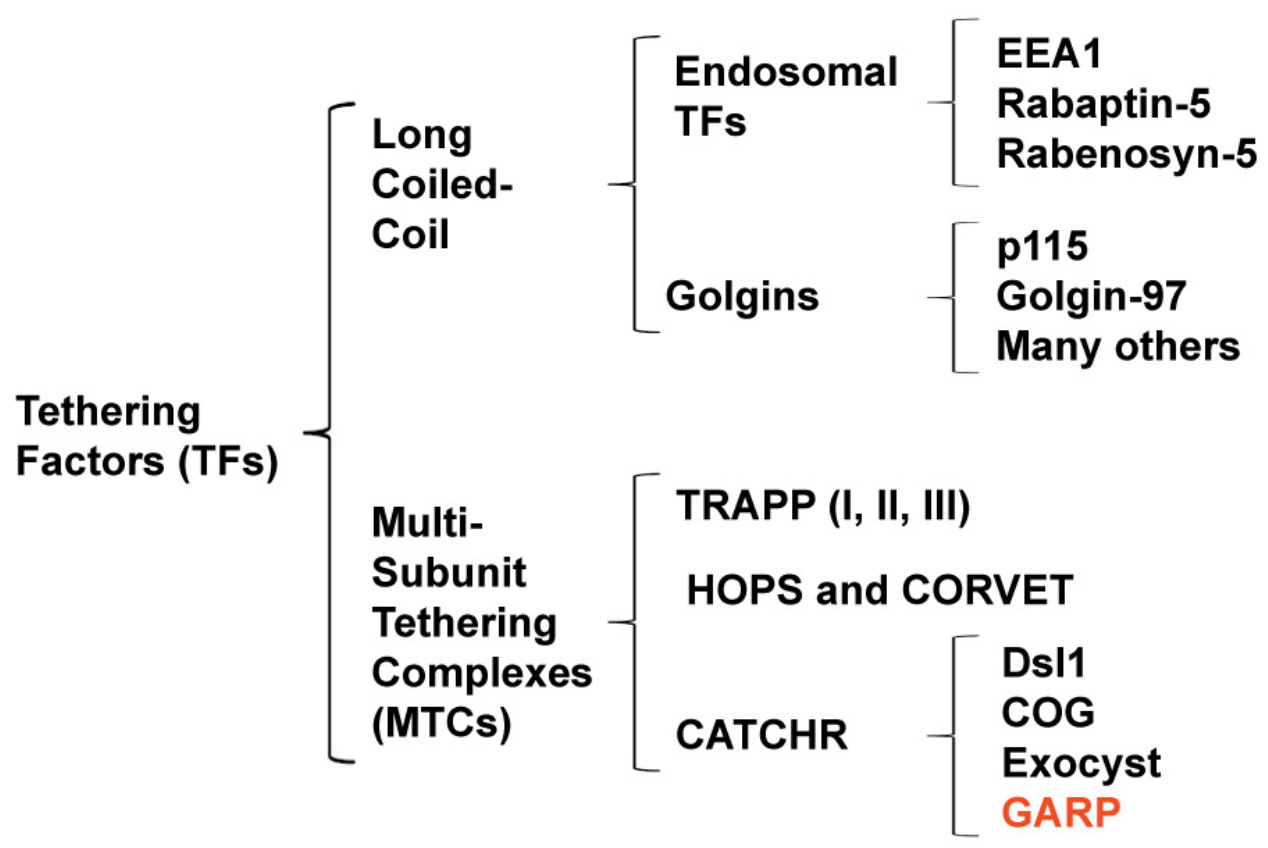

Figure IX.2. Classification of tethering factors based on their structure. This classification is based on (Cai et al, 2007; Yu \& Hughson, 2010). CATCHR, complex associated with tethering containing helical rods; COG, the conserved oligomeric Golgi; CORVET, class C core vacuole/endosome tethering complex; Ds11, dependence on SLY1-20; EEA-1, early endosome antigen-1; GARP, Golgi-associated retrograde protein complex; HOPS, homotypic fusion and vacuole protein sorting; MTC, multi-subunit tethering complex; TF, tethering factor; TRAPP, transport protein particle. Source: picture based on (Bonifacino \& Hierro, 2011), with permission from Elsevier. 
There are at least three forms of the TRAPP complex described, which comprise distinct and overlapping subunits (Lynch-Day et al, 2010; Sacher et al, 2008). In yeast, these complexes function in endoplasmic reticulum (ER) to Golgi transport and also act at the trans-Golgi network (TGN) and at phagosomes (Lynch-Day et al, 2010; Sacher et al, 2008). It has also been shown that yeast TRAPP complexes possess GEF activity towards the Rab proteins, Ypt1p and Ypt31/32p (Jones et al, 2000).

The HOPS and CORVET complexes possess four common subunits, whereby each complex contains two additional subunits (Peplowska et al, 2007; Wurmser et al, 2000). In yeast it has been demonstrated that the CORVET complex binds to the Rab5like GTPase Vps21 at the endosome, whereas the HOPS complex interacts with Ypt7/Rab7 at the late endosomes (Epp et al, 2011).

The CATCHR subgroup consists of structurally related complexes (Figure IX.3), such as Dsl1 (dependence on SLY1-20), the COG (conserved oligomeric Golgi) complex, the exocyst, and the GARP (Golgi-associated retrograde protein) complex (Yu \& Hughson, 2010).

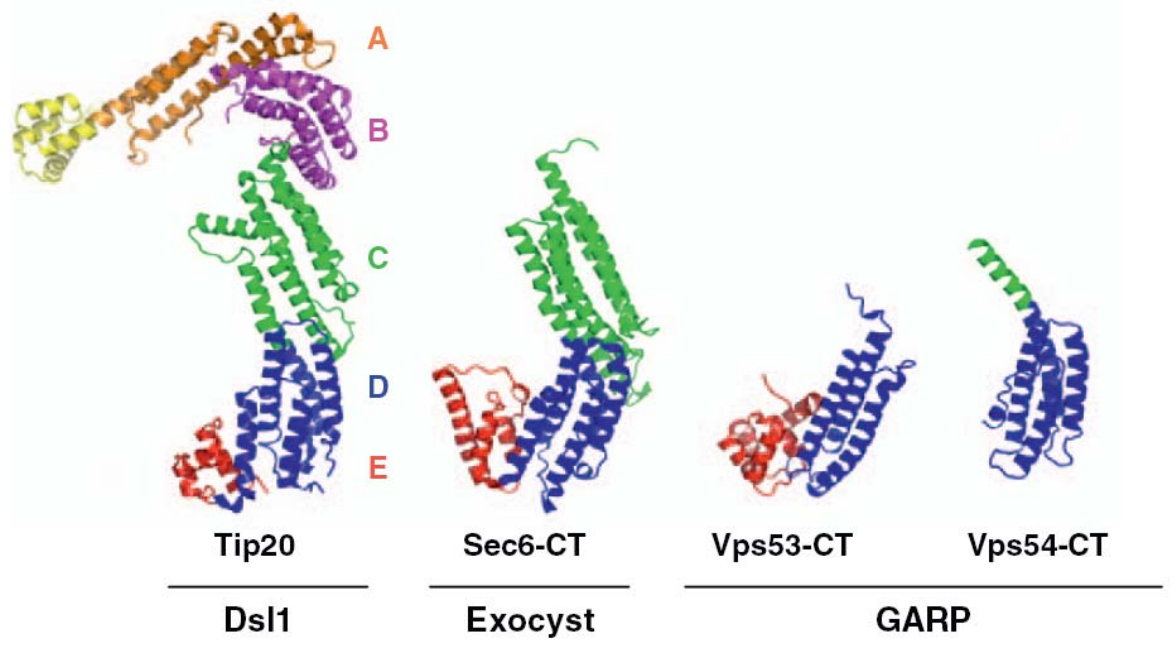

Figure IX.3. The CATCHR subgroup of multi-subunit tethering complexes is structurally related. Crystal structures of C-terminal fragments from yeast Vps53 (Vasan et al, 2010) and human Vps54 (Perez-Victoria et al, 2010a) and the yeast Sec6 subunit of the exocyst complex (Sivaram et al, 2006) and the full-length yeast Tip20 subunit of the Dsll complex (Tripathi et al, 2009) are shown. Structures are displayed as ribbon diagrams with the tandem a-helical-bundle domains (A-E) represented in different colors. Source: reprinted from (Bonifacino \& Hierro, 2011), with permission from Elsevier. 
The Dsl1 complex has only three subunits and is important for the retrograde transport from the Golgi complex to the ER (Kamena \& Spang, 2004; Kraynack et al, 2005). The exocyst is formed by eight subunits, which are required for trafficking of Golgi-derived vesicles to the plasma membrane (He \& Guo, 2009). The COG complex is formed by eight subunits exhibiting distinct functions (Ungar et al, 2002). It has been shown that the COG complex is involved in the retrograde transport from both endosome to Golgi and within the Golgi (Bruinsma et al, 2004; Ram et al, 2002; Whyte \& Munro, 2001). Furthermore, the COG complex also functions in the transport of ER-derived vesicles to the Golgi (VanRheenen et al, 1998; VanRheenen et al, 1999). The role of the GARP complex is described in the next section. Figure IX.4 summarizes the sites of action of the above mentioned MTCs.

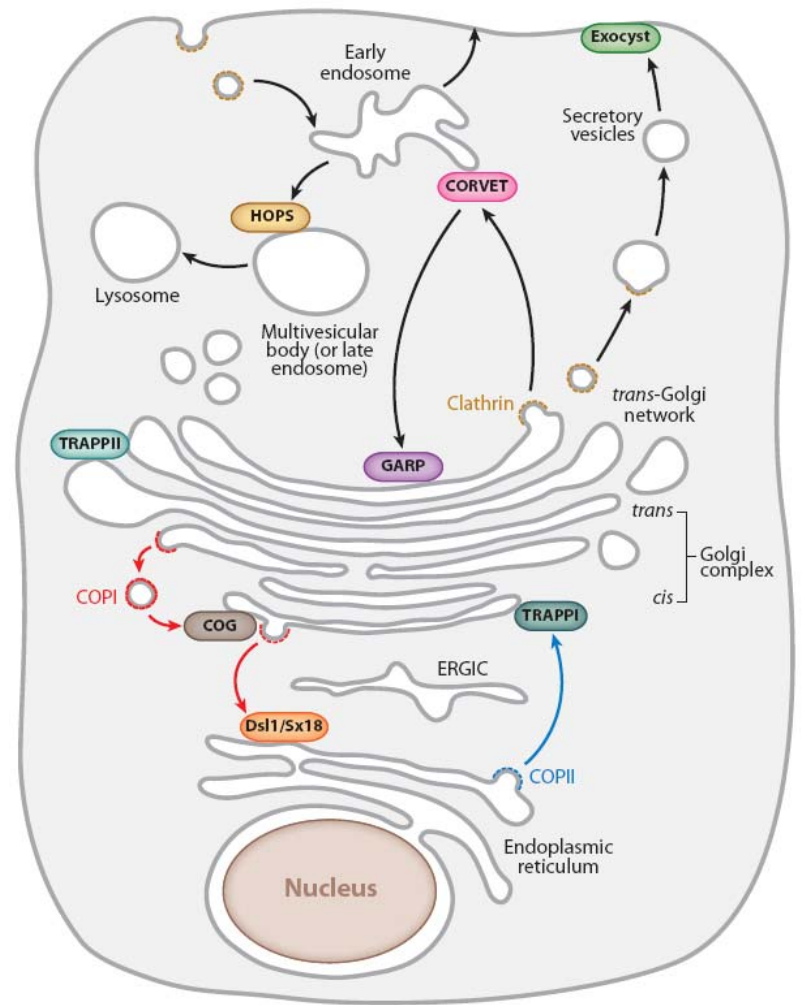

Figure IX.4. Different transport pathways are mediated by multi-subunit tethering complexes. COG, conserved oligomeric Golgi complex; CORVET, class C core vacuole/endosome tethering complex; ERGIC, endoplasmic reticulum-Golgi intermediate compartment; GARP, Golgi associated retrograde protein complex; HOPS, homotypic fusion and vacuole protein sorting; CORVET, class $\mathrm{C}$ vacuolar protein sorting; TRAPP, transport protein particle. Source: picture taken from ( $\mathrm{Yu} \&$ Hughson, 2010), with permission from ANNUAL REVIEW OF CELL AND DEVELOPMENTAL BIOLOGY. 


\section{IX.3 The GARP complex in retrograde trafficking}

The GARP tethering complex is evolutionarily conserved from yeast to mammals and consists of four subunits, termed Vps51, Vps52, Vps53, Vps54 (Luo et al, 2011; Perez-Victoria et al, 2010b; Siniossoglou \& Pelham, 2002). In yeast the GARP complex is localized to the TGN to tether retrograde vesicles derived from endosomes to the post Golgi (Conibear et al, 2003; Conibear \& Stevens, 2000; Siniossoglou \& Pelham, 2001; Siniossoglou \& Pelham, 2002). This retrograde transport facilitates the recycling of cargo material such as Vps10, a transmembrane sorting receptor for hydrolase precursors, and the v-SNARE Snc1 (Conibear \& Stevens, 2000; Quenneville et al, 2006; Siniossoglou \& Pelham, 2001; Siniossoglou \& Pelham, 2002). Like in yeast, mammalian and C. elegans GARP was found to be located to the TGN but also at endosomes (Ho et al, 2006; Liewen et al, 2005; Luo et al, 2011; Perez-Victoria et al, 2008; Perez-Victoria et al, 2010b). Human GARP is required for the retrograde transport of mannose-6-phosphate receptors as well as TGN-resident proteins and some SNAREs, supporting a general role in endosome to TGN transport (Perez-Victoria et al, 2008; Perez-Victoria et al, 2010b).

It is believed that the GARP complex simultaneously binds to the incoming vesicles and to the acceptor membrane achieved by interactions with SNARE proteins and small GTPases of both Rab and Arl families (Bonifacino \& Hierro, 2011) (Figure IX.5). In yeast, the subunit Vps52 interacts with Ypt6/Rab6 (Siniossoglou \& Pelham, 2001), and Vps53 with Arl1p (Panic et al, 2003). The yeast subunit Vps51 interacted with the t-SNARE, Tlg1 (Conibear et al, 2003; Siniossoglou \& Pelham, 2002). This binding seems to be conserved because human Vps51 also interacts with the human ortholog of Tlg1, Syntaxin-6 (Perez-Victoria et al, 2010b). Furthermore, Vps53 and Vps54 also bind to SNAREs, such as Syntaxin-16 and Vamp4, which are involved in endosome to TGN transport (Perez-Victoria \& Bonifacino, 2009). Interestingly, it has been shown that the loss of GARP caused reduced formation of TGN SNARE complexes (Perez-Victoria \& Bonifacino, 2009). Thus, GARP might contribute to the TGN SNARE assembly and/or stabilization (Perez-Victoria \& Bonifacino, 2009). In consistent with this, all C. elegans GARP subunits bound to a specific set of Golgi SNAREs by using the yeast two-hybrid 
system (Luo et al, 2011). Thus, it is likely that the GARP complex facilitates retrograde trafficking to the Golgi though various SNARE interactions.

A

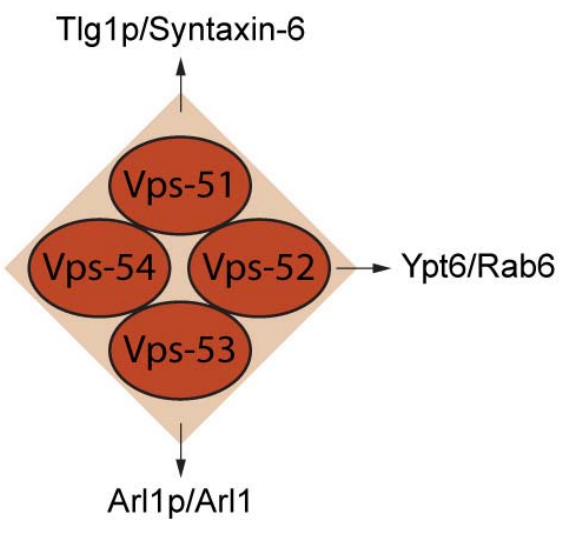

B

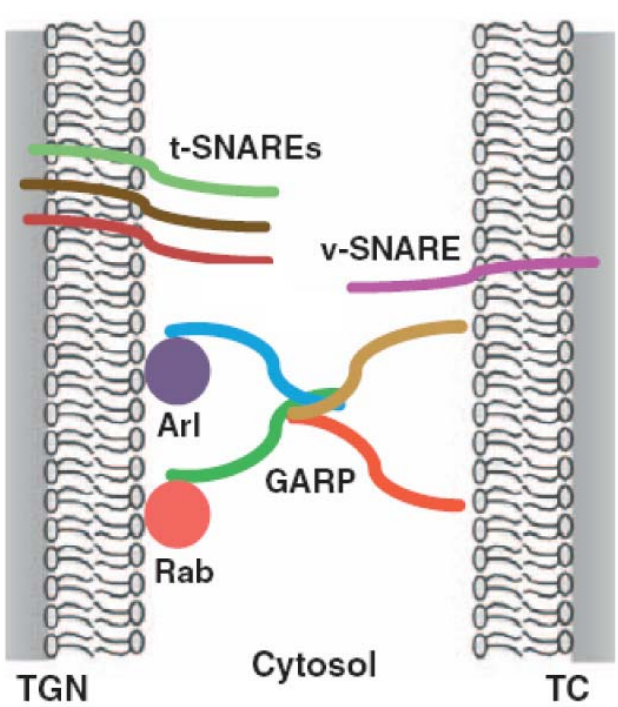

Figure IX.5. The GARP complex interacts with small GTPases and SNARE proteins during retrograde trafficking. (A) Schematic representation of the GARP complex. Subunits that interact with SNARE proteins and small GTPases are indicated. Names of interaction partners in yeast have been used. Source: picture based on (Oka \& Krieger, 2005). (B) Speculative representation of tethering mediated by the GARP complex. GARP is shown as a heterotetramer complex assembled through the N-terminal regions of its subunits. The interaction with small GTPases might contribute to GARP recruitment to the TGN, whereby binding to SNAREs might promote SNARE complex formation. Source: reprinted from (Bonifacino \& Hierro, 2011), with permission from Elsevier.

Besides the role in retrograde trafficking from endosomes to the TGN, it has been shown that the GARP complex is also involved in other cellular processes, such as autophagy, mitochondrial morphology and function, motor neuron survival, spermiogenesis as well as embryonic development and viability [reviewed in (Bonifacino \& Hierro, 2011)]. 


\section{IX.4 The role of the GARP subunit, Vps51, in C. elegans}

Previously, we have characterized the subunits of the GARP complex in $C$. elegans (Luo et al, 2011). Like in mammals (Liewen et al, 2005), orthologs of yeast Vps52, Vps53, Vps-54 were readily identified in C. elegans by sequence homology searches (www.wormbase.org). The identification of Vps51 in mammals and C. elegans turned out to be more difficult, since the metazoan Vps51 exhibits low sequence similarities and is much larger ( $\geq 700 \mathrm{aa})$ than its yeast counterpart ( $150 \mathrm{aa})$ (Ho et al, 2006; Luo et al, 2011; Perez-Victoria et al, 2010b). Remarkably, mutations in the subunit Vps51 in yeast and metazoans caused less severe defect phenotypes compared to mutations in the remaining subunits Vps52-54, e.g. changes in vacuolar/lysosomal morphology (Luo et al, 2011; Siniossoglou \& Pelham, 2002). Furthermore, the formation and stability of the yeast GARP complex in yeast did not depend on the subunit Vps51, but on the remaining core subunits (Conibear \& Stevens, 2000; Siniossoglou \& Pelham, 2002). All these findings suggest that Vps51 might be an auxiliary subunit. It might be required for more specialized functions of the GARP complex in multi-cellular organisms (Ho et al, 2006; Luo et al, 2011; Perez-Victoria et al, 2010b). In agreement with this, Vps51 seems to be the subunit that is most modified during evolution compared to the remaining GARP subunits in order to adapt to the newly specialized tasks. These functions might have been dispensable in yeast, which led to a shortened minimal Vps51 protein.

\section{IX.5 Aims of this study}

This chapter describes a novel role for the GARP complex in C. elegans. We studied its involvement in DCV maturation. We further investigated possible interactions with Rab GTPases in C. elegans. Moreover, we provide explanations how the GARP complex in cooperation with RAB-2 and its effectors might contribute to DCV maturation. 
The results presented in chapter 3 were performed by the author of this thesis with the help of a lab rotation student, Katharina Lindner. Experiments where the author received help are indicated in the figure legends. 


\section{Chapter 3}

\section{Results}

\section{X.1 The GARP complex is involved in DCV trafficking}

Through our previous work to characterize retrograde trafficking events within the Golgi-endosomal interface we characterized the subunits of the Golgi-associated retrograde protein (GARP) tethering complex (Luo et al, 2011). The GARP complex is known to be required for tethering of endosome-derived vesicles to the late Golgi during retrograde transport (Conibear \& Stevens, 2000). Similar to humans and yeast (PerezVictoria et al, 2010; Siniossoglou \& Pelham, 2002), it has been shown that the C. elegans GARP complex also consists of four subunits: VPS51, VPS52, VPS53, VPS54 (acuolar

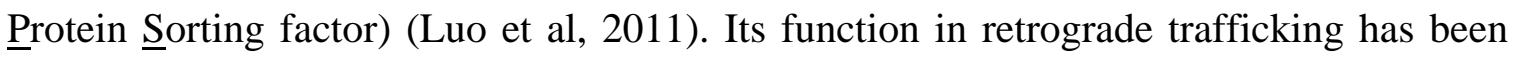
characterized in C. elegans and other organisms (Bonifacino \& Hierro, 2011; Luo et al, 2011). C. elegans mutant alleles for each subunit exist, such as vps-51(tm4275), vps52(ok853), vps-53(ok2864), and vps-54(ok1463), which were used in this study (www.wormbase.org) (Luo et al, 2011).

During our initial phenotypic characterization of mutants in the GARP complex subunits we realized that GARP mutants exhibited strongly decreased locomotion by about $50 \%$ as compared to wild type animals (Figure X.1). This suggests that the GARP complex functions in neurons and might be required for normal locomotion. Consistent with this hypothesis, vps genes are ubiquitously expressed with strong expression in 
neurons in C. elegans (Luo et al, 2011). Interestingly, the locomotion of mutants in the subunits of the GARP complex (vps-51, -52, -53 and -54) strongly resembled the slow, "unmotivated" locomotion of unc-108/rab-2 mutant animals. Mutations in the GARP subunits vps-52 and vps-53 were also recovered in screens for mutants displaying unc108/rab-2-like unmotivated locomotion phenotypes (M. Ailion, unpublished results).

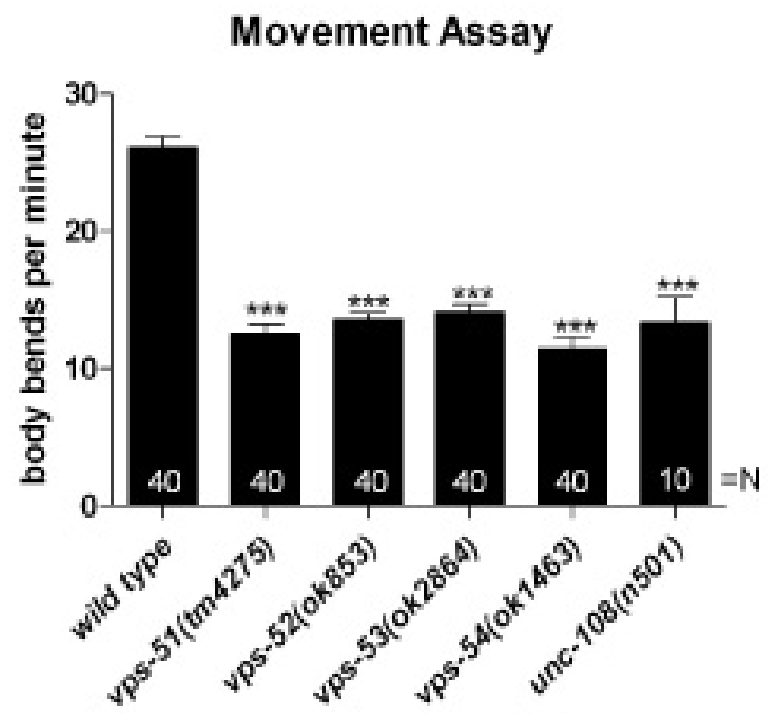

Figure X.1. GARP mutants display movement defects. Young adult worms of each strain were transferred to non-seeded plates, adjusted for several minutes before the number of body bends per min of each worm was recorded. Like unc-108/rab-2 mutants, movement rates of GARP mutants were decreased by about $50 \%$ to wild type animals: for vps-51(tm4275) mutants 12.54 \pm 0.69 ; for vps-52(ok853) mutants $13.57 \pm 0.51$; for vps-53(ok2864) mutants $14.14 \pm 0.51$; for vps-54(ok1463) mutants 11.41 \pm 0.85 ; for unc-108(n501) mutants $13.33 \pm 1.91$ and for wild type animals $26.08 \pm 0.78$ body bends per minute were counted. Error bars = s.e.m. ( ${ }^{* * *}, \mathrm{P}<0.0001$; Student's t-test). Source: this experiment was conducted by Suekyoung Jeon and Katharina Lindner in our lab. The original data was kindly provided by them.

Due to the observed slow locomotion rates and similarities to unc-108/rab-2 mutants we wondered whether the GARP complex might also be involved in DCV trafficking or maturation. We subjected the GARP mutants to the DCV assay and analyzed them for trafficking defects of the DCV marker NLP-21-VENUS (Sieburth et al, 2007). We observed decreased fluorescence levels of NLP-21-derived VENUS in the dorsal nerve cord (DNC) and in coelomocytes of all GARP subunits mutants (Figure 
X.2). vps-51(tm4275) mutants showed $12.66 \pm 2.36 \%$, vps-52(ok853) mutants $8.70 \pm 2.51 \%$ and vps-53(ok2864) mutants 9.87 $\pm 2.17 \%$ VENUS fluorescence in their axons compared to wild type animals $100 \pm 8.90 \%$ (Figure X.2). Surprisingly, unlike the other GARP subunits, vps-54(ok1463) mutants displayed a less severe defect in VENUS trafficking to the DNC, with $61.07 \pm 8.32 \%$ VENUS fluorescence in the DNC compared to wild type.

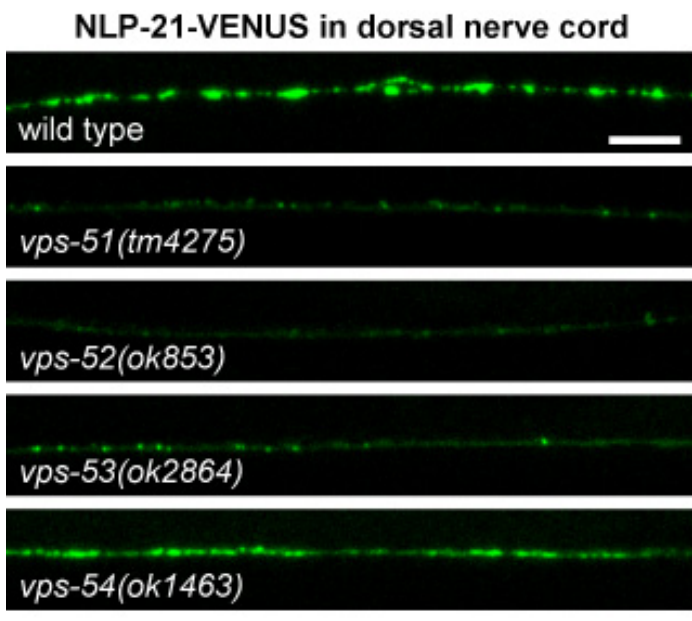

NLP-21-VENUS in dorsal nerve cord

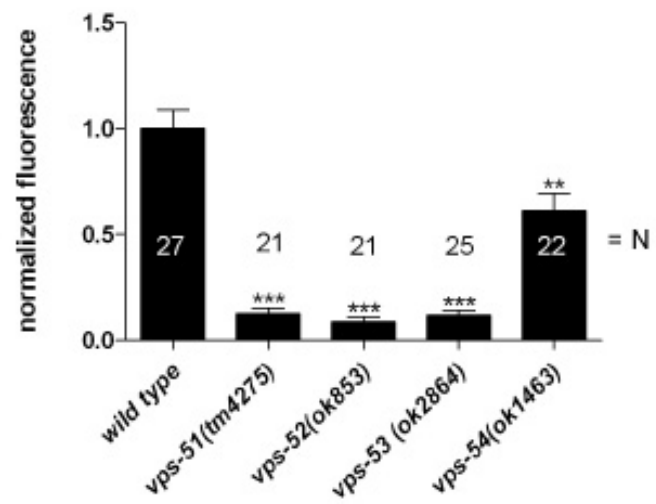

Figure X.2. GARP mutants show defects in DCV trafficking in the DNC. vps-51(tm4275), vps-52(ok853), and vps-53(ok2864) mutants displayed similar decreased NLP-21-derived VENUS fluorescence in the DNC, whereas vps-54(ok1463) mutants showed a less severe DCV phenotype. Scale bar represent $5 \mu \mathrm{m}$. Error bars = s.e.m. $(* *, \mathrm{P}<0.001$; $* * *, \mathrm{P}<0.0001$; Student's t-test).

Despite the morphological defects observed in lysosomal compartments of GARP mutants, the fluid phase uptake and the degradation of endocytosed material were shown not to be strongly affected by this (Fares \& Greenwald, 2001; Luo et al, 2011). Therefore, we can utilize the coelomocyte VENUS uptake to assay NLP-21-derived VENUS secretion from the DNC in these mutants. Consistent with the observed VENUS fluorescence in the DNC, vps-52 and vps-53 deletion mutants showed a decrease in VENUS fluorescence in their coelomocytes (Figure X.3). Surprisingly, in vps-51 mutants higher levels of endocytosed VENUS fluorescence in coelomocytes compared to the DNC were detected (Figure X.3), suggesting lower rates in VENUS degradation in coelomocytes, which would result in accumulation of VENUS within endocytic 
compartments. Furthermore, in vps-54 mutants almost no VENUS fluorescence in their coelomocytes could be detected (Figure X.3), whereas about 60\% VENUS fluorescence in the DNC compared to wild type animals were present (Figure X.2). This suggests that vps-54 mutants might harbor additional defects in the regulation of DCV secretion or defects in VENUS uptake within coelomocytes that have to be further studied.

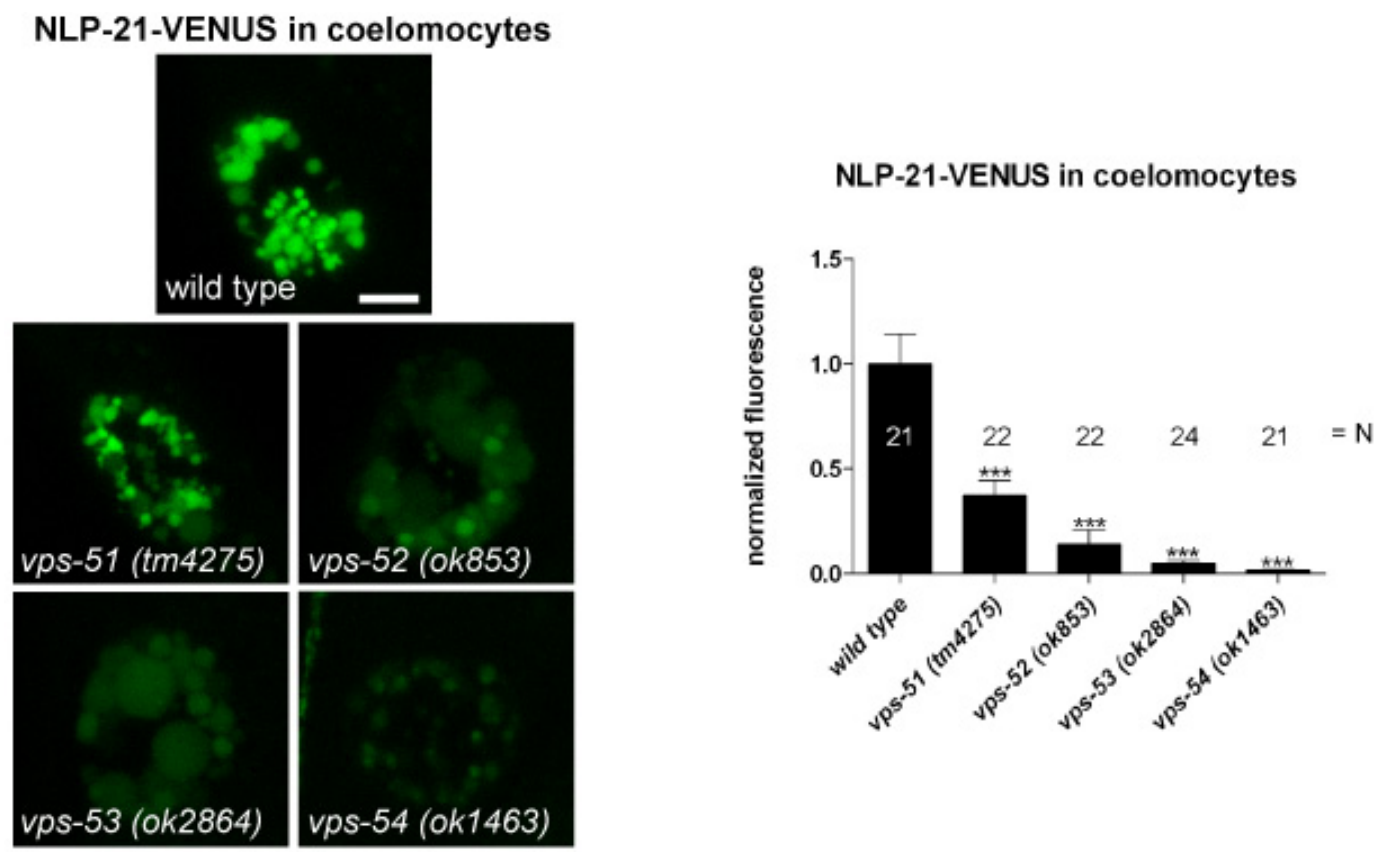

Figure X.3. GARP mutants show less fluorescence of endocytosed VENUS in the coelomocytes. Less VENUS fluorescence in coelomocytes was detected in all vps-mutants compared to wild type worms. However, vps-51 mutants displayed higher fluorescence levels of endocytosed VENUS in their coelomocytes compared to the VENUS present in their DNC, whereas vps-54 mutants displayed the opposite phenotype (Figure X.2). Scale bar represent $5 \mu \mathrm{m}$. Error bars $=$ s.e.m. $(* * *, \mathrm{P}<0.0001$; Student's t-test).

It is possible that GARP mutants display general membrane trafficking defects in neurons leading to decreased NLP-21-derived VENUS fluorescence in their axons. In order to test whether only DCV trafficking and not SV trafficking, is specifically affected in GARP mutants, we analyzed the localization of the synaptic marker proteins, RAB-3 and Synaptobrevin-1 (SNB-1), using integrated strains expressing YFP-RAB-3 and GFPSNB-1 in cholinergic motoneurons (Sieburth et al, 2005). The fluorescence of YFP-RAB-

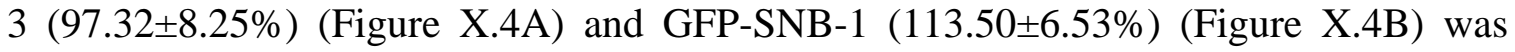


unchanged in the DNC of vps-53(ok2864) or vps-52(ok853) mutants, respectively, when compared to wild type axons. These findings indicate that SV trafficking might not be affected in GARP mutants. In order to detect more subtle changes in SV and DCV morphology and localization, vps-mutants are currently being investigated by highpressure freeze electron microscopy in our lab. In this way, we further attempt to analyze the synaptic ultrastructure of the GARP mutants.

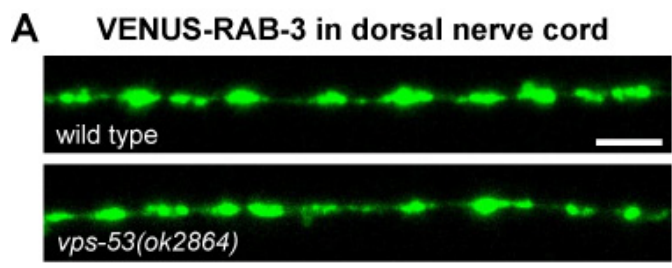

VENUS-RAB-3 in

dorsal nerve cord

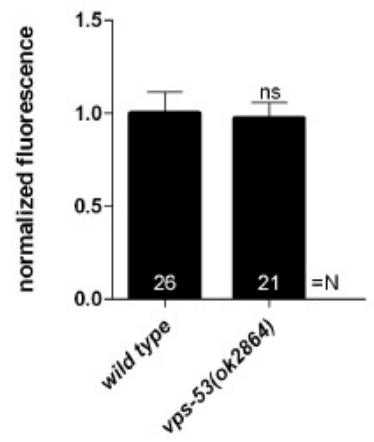

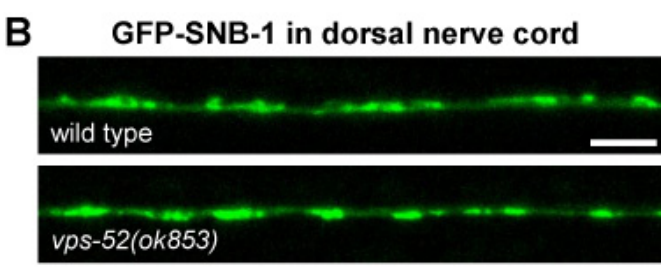

GFP-SNB-1 in dorsal nerve cord

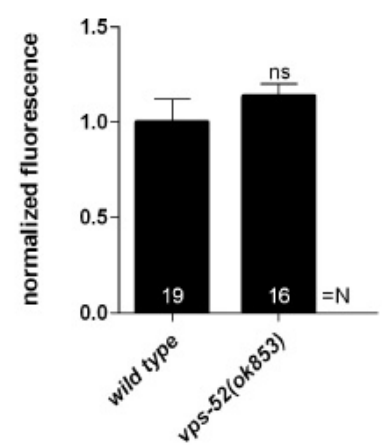

Figure X.4. GARP mutants display no obvious defects in synaptic vesicle trafficking. The transport of synaptic vesicles to the synapses in the DNC was visualized by VENUS-tagged RAB-3 (VENUS-RAB-3) (A) and GFP-tagged synaptobrevin (GFP-SNB-1) (B). No differences were observed in transport of these SV markers in vps-53(ok2864) or vps-52(ok853) mutants, respectively. Scale bar represents $5 \mu \mathrm{m}$. Error bars $=$ s.e.m. (ns, P $>0.05$; Student's t-test).

\section{X.2 GARP subunits interact with individual RAB GTPases in Y2H}

In order to regulate vesicle fusion to the donor membrane, tethering factors are known to function as effector proteins of Rab GTPases (Cai et al, 2007; Yu \& Hughson, 2010). To test whether the GARP complex acts in cooperation with these small GTPases in DCV trafficking, we attempted to identify possible GARP-RAB interactions in $C$. elegans. Therefore, we tested the four VPS proteins against all C. elegans Rab GTPases 
using the Y2H system (Figure X.5). We detected weak interaction between the GARP subunit VPS-51 with activated, GTP-bound RAB-8 and RAB-19, whereas VPS-53 specifically bound to activated RAB-30. Surprisingly, VPS-52 and VPS-54 did not bind to any analyzed $\mathrm{RAB}$ proteins within the $\mathrm{Y} 2 \mathrm{H}$ system. The identified RAB interaction partners of the GARP subunits VPS-51 and VPS-53 do not display defects in locomotion or DCV trafficking (Nikhil Sasidharan, unpublished data; chapter 1 this study). Therefore, these interactions might play a role in GARP functions besides DCV trafficking.
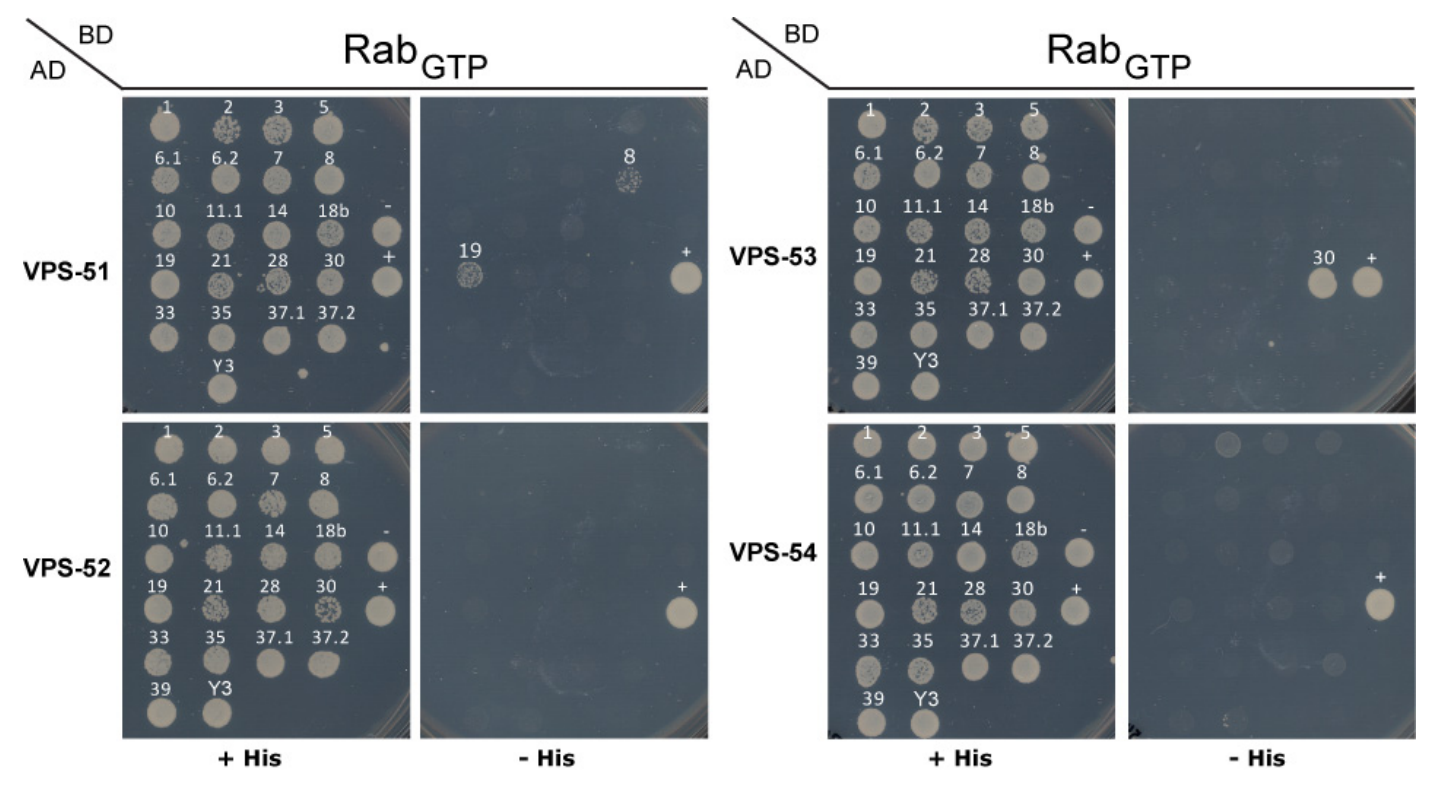

Figure X.5. The GARP subunits VPS-51 and VPS-53 interact with different RAB GTPases in a Y2H screen. All C. elegans RAB proteins in their constitutively GTP-bound form were tested for interaction with all four GARP subunits. VPS-51 weakly interacted with GTP-bound RAB-8 and RAB-19, whereas VPS-53 bound to activated RAB-30. No interactions between RAB GTPases and the subunits VPS-52 or VPS-54, respectively, were detected in this Y2H screen. $\mathrm{AD}$, Gal4p DNA activation domain fusion; BD, Gal4p DNA binding domain fusion; His, histidine; $\mathrm{RAB}_{\mathrm{GTP}}$, constitutively GTP-bound RAB GTPase; “+” represents internal positive control; "-” represents internal negative control. Note: VPS-51 was not tested against GTP-bound RAB-39, which still has to be done. Source: this experiment was conducted by the lab rotation student Katharina Lindner in our lab. 


\section{X.3 The GARP subunit VPS-51 interacts with RAB-2 effectors, RIC-19 and RUND- 1 as well as with the putative RAB-2 GAP, TBC-8}

Since we could show that the GARP subunits play a role in DCV trafficking, we analyzed interactions of VPS-51 with the novel DCV players, TBC-8, RIC-19 and RUND-1 via Y2H (Figure X.6A). Interestingly, all these three proteins interacted with VPS-51 in Y2H. In addition, we detected interaction between the GARP subunit VPS-53 and TBC-8. Due to self-activation properties of all three proteins, RIC-19, RUND-1 and VPS-53, when expressed as the DNA-binding domain-fusion (data not shown), these interactions could not be studied by Y2H. The binding affinity of VPS-51 to TBC-8, RIC-19 and RUND-1 were confirmed by co-IP experiments (Figure X6B).

A

yeast two-hybrid

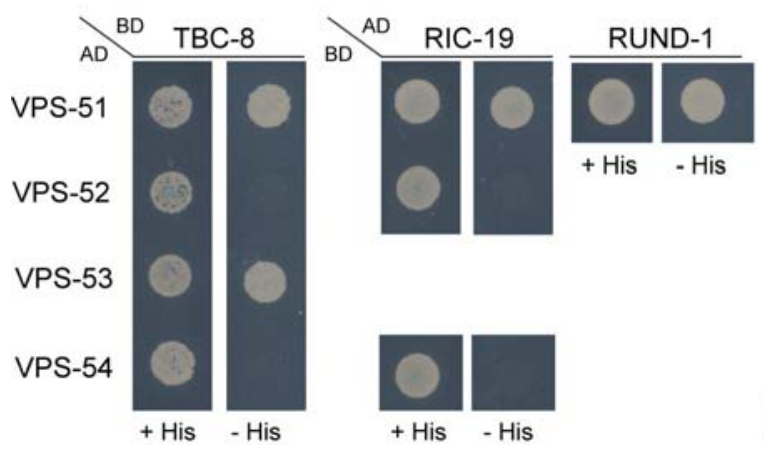

B

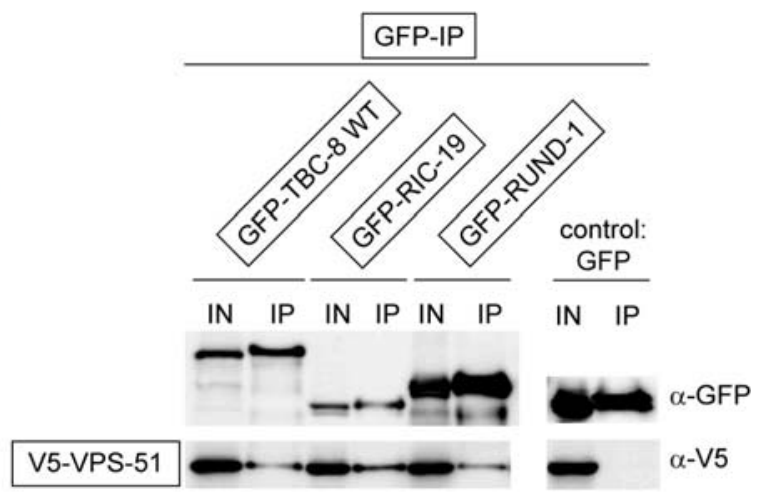

Figure X.6. The GARP subunit VPS-51 interacts with TBC-8, RIC-19 and RUND-1 in Y2H and in co-IP experiments. (A) Y2H analysis revealed that TBC-8 interacts with VPS-51 and VPS-53, whereas no interactions between TBC-8 with VPS-52 and VPS-54 were detected. Furthermore, VPS-51 also bound to the RAB-2 effectors, RIC-19 and RUND-1. Due to selfactivation properties of VPS-53 and RIC-19 when expressed as a DNA-binding domain-fusion, this interaction could not be performed. Interaction analysis between RUND-1 and VPS-52, -53, 54 were not conducted in this study. (B) HEK293 cells were co-transfected with constructs expressing GFP-tagged TBC-8, RIC-19 and RUND-1 (or GFP alone as control) and V5-VPS-51. An anti-GFP antibody was used to precipitate GFP-tagged fusion proteins or GFP as control (GFP-IP). Interactions were visualized on Western blots. V5-tagged VPS-51 bound to all three tested GFP-fusion proteins, GFP-TBC-8, GFP-RIC-19 and GFP-RUND-1. AD, Gal4p DNA activation domain fusion; BD, Gal4p DNA binding domain fusion; His, histidine; IN, Input; IP, immunoprecipitation. 
In order to test which domain of TBC-8 is required for the interaction with VPS51, we performed a domain mapping analysis. We studied the binding properties of VPS51 to the detectable protein domains of TBC-8 within the Y2H system (Figure X.7A). The interaction could be mapped to the RUN-domain of TBC-8 with VPS-51 determined by Y2H and co-IP (Figure X.7).

A

yeast two-hybrid

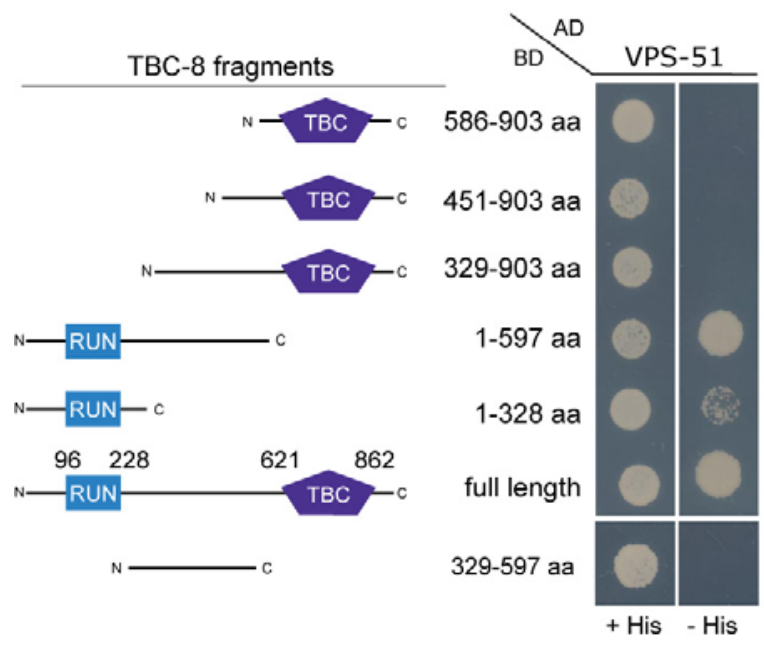

B co-immunoprecipitation

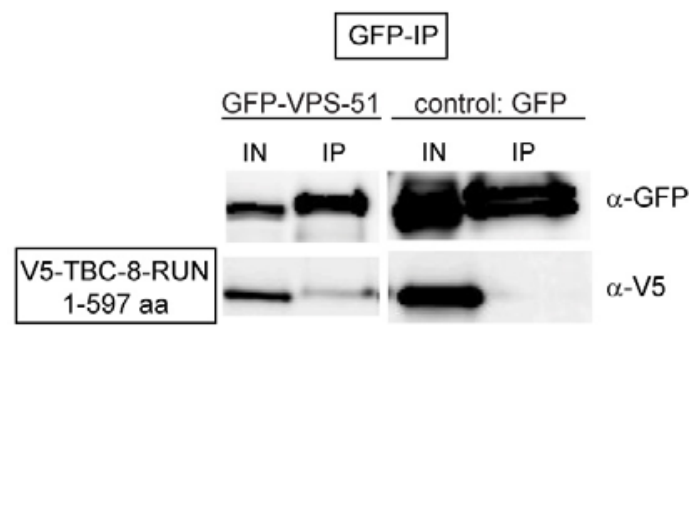

Figure X.7. VPS-51 interacts with the RUN-domain of TBC-8 in Y2H and in co-IP experiments. (A) Domain mapping experiment revealed that VPS-51 interacts with TBC-8 fragments harboring the RUN-domain, whereas VPS-51 did not bind to the TBC-domain of TBC8. (B) Interaction between VPS-51 and the RUN-domain of TBC-8 were confirmed by co-IP analysis. HEK293 cells were co-transfected with constructs expressing GFP-tagged VPS-51 (or GFP alone as control) and a V5-tagged RUN-domain of TBC-8 (1-597 aa). An anti-GFP antibody was used to precipitate GFP-VPS-51 or GFP as control (GFP-IP). Interactions were visualized on Western blots. AD, Gal4p DNA activation domain fusion; BD, Gal4p DNA binding domain fusion; His, histidine; IN, Input; IP, immunoprecipitation.

\section{X.4 The GARP subunit VPS-51 and RUND-1 act in the same pathway}

Interaction studies revealed that the GARP complex binds to RAB-2 effectors, RUND-1 and RIC-19 and to the putative RAB-2 GAP, TBC-8. In order to test whether VPS-51 acts together with these factors in the same pathway, we constructed double mutants of these players and subjected them to the NLP-21-VENUS assay. 
Unfortunately, we were not able to obtain double mutants for ric-19 and vps-51, because both genes are located on chromosome I, only separated by $2 \mathrm{Mb}$ (www.wormbase.org). Future RNAi experiments should overcome this genetic linkage issue.

Double mutants of $v p s-51$ and $t b c-8$ caused an additive defect (6.58 $\pm 1.34 \%)$ in the decrease of VENUS fluorescence in the dorsal nerve cord, compared to vps-51 (10.92 $\pm 1.30 \%)$ and $t b c-8(24.96 \pm 2.64 \%)$ single mutants (Figure X.8). This suggests that the GARP complex and the putative RAB-2 GAP act in parallel pathways during DCV maturation. In contrast, double mutants of vps-51 and rund-1 revealed that these proteins act in the same pathway, since double mutants displayed similar defects in the NLP-21derived VENUS fluorescence in the DNC (10.55 $\pm 1.53 \%)$ like vps-51 (10.92 $\pm 1.30 \%)$ and rund-1 (13.41士1.64\%) single mutants (Figure X.8).

\section{NLP-21-VENUS in dorsal nerve cord}

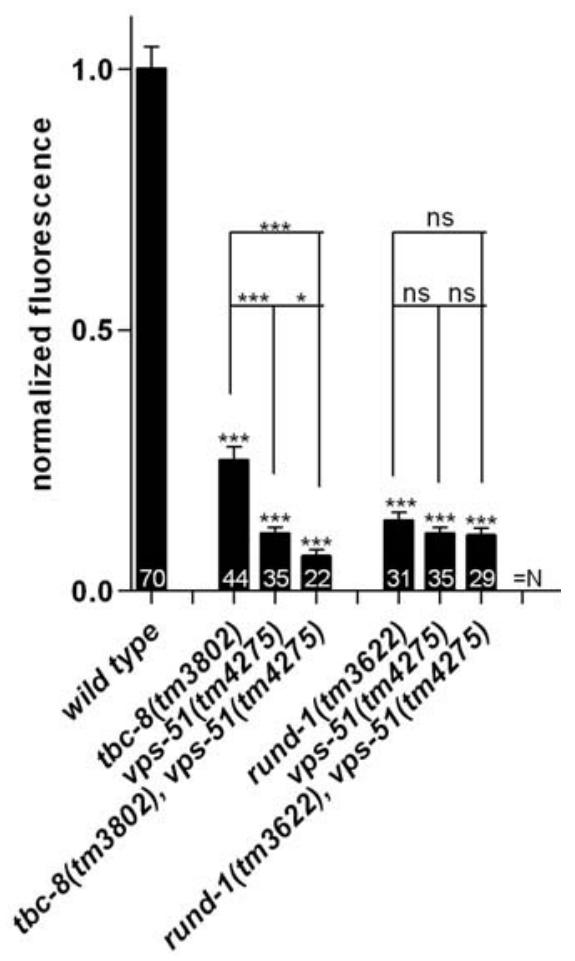

Figure X.8. VPS-51 and RUND-1 act in the same pathway during DCV trafficking, whereas VPS-51 and TBC-8 do not. Double mutant analysis of vps-51 with tbc-8 and rund-1 are depicted. Single mutants of $t b c-8(24.96 \pm 2.64 \%)$ and vps-51 (10.92 $\pm 1.30 \%)$ displayed a less severe NLP-21-VENUS phenotype than double mutants of both (6.58 $\pm 1.34 \%)$, suggesting that TBC-8 and VPS-51 act in parallel pathways contributing to DCV trafficking. RUND-1 and VPS51 act in the same pathway, since double mutants of both genes did not lead to additive effects of NLP-21-derived VENUS fluorescence at the DNC. Error bars = s.e.m. (ns, P > 0.05; *, P < 0.05; $* * *, \mathrm{P}<0.0001$; Student's t-test). 


\section{Chapter 3}

\section{Discussion}

\section{XI.1 Function of the retrograde transport during DCV maturation}

The involvement of the multisubunit Golgi-associated retrograde protein (GARP) tethering complex in retrograde trafficking between endosomes and post Golgi compartments has been characterized in C. elegans and other organisms (Bonifacino \& Hierro, 2011; Luo et al, 2011). Its function in tethering of endosome-derived vesicles to the trans-Golgi during retrograde transport is described (Conibear \& Stevens, 2000). Since the transport of TGN cargo receptors and the localization of resident TGN membrane proteins, such as TGN46, are impaired in GARP mutants, it was proposed that the GARP complex plays a general role in the delivery of retrograde cargo (Conibear \& Stevens, 2000; Perez-Victoria et al, 2008; Siniossoglou \& Pelham, 2002).

Previously, it has been shown that loss of the GARP complex caused alterations in lysosomal morphology (Conboy \& Cyert, 2000; Perez-Victoria et al, 2008). In C. elegans GARP mutants, severely enlarged lysosomes were observed (Luo et al, 2011). A reason for this phenotype might be the disturbed transport from endosomes to the Golgi resulting into an increased membrane flow into the lysosomal pathway (Luo et al, 2011). Despite the detected morphological changes of lysosomes, we could demonstrate that these compartments are functional in degrading endocytosed cargo (Luo et al, 2011).

Here, we identified an additional function of the GARP complex in C. elegans neurons. Mutants in the subunits of the GARP complex, vps-51, $-52,-53$ and -54 , 
displayed a slow unmotivated locomotion as compared to wild type animals. Surprisingly, this locomotion phenotype resembled the slow, unmotivated locomotion of unc-108/rab-2 mutant animals (Chun et al, 2008; Edwards et al, 2009; Sumakovic et al, 2009). Thus, we predict that the GARP complex might also be required for normal locomotion like RAB-2 in C. elegans (Edwards et al, 2009; Sumakovic et al, 2009). Interestingly, mutations in the GARP subunits vps-52 and vps-53 were also identified in a screen for mutants displaying unc-108/rab-2-like unmotivated locomotion phenotypes (M. Ailion, unpublished results). Using the NLP-21-VENUS assay, we studied the DCV trafficking effects in mutants of the GARP complex subunits vps-51, $-52,-53$ and -54 . This analysis revealed that the GARP complex mutants displayed the same RAB-2 like DCV maturation defects. The GARP complex is specifically involved in DCV trafficking, since GARP mutants did not display defects in the trafficking of SV-markers, RAB-3 and Synaptobrevin-1 (SNB-1) to axons, which suggest that SV trafficking might not be affected in GARP mutants. Future electron microscopy analysis of these mutants will reveal potential subtle defects in the morphology, number and size of SVs and DCVs at synaptic zones.

In this study we could show that the GARP complex exhibited defects in DCV trafficking, indicating that the retrograde transport of cargo from endosomes to the Golgi is essential for proper DCV maturation. To our knowledge, such a necessity of membrane cycling between the Golgi and endosomes for DCV maturation was not described before. However, it is conceivable that mis-sorted cargo and TGN-resident proteins, such as receptors have to be recycled back to the Golgi to ensure cycling of the pathway. Since the GARP mutants and unc-108/rab-2 mutants exhibited similar uncoordinated locomotion and similar decreased levels in VENUS cargo at the axons, it is tempting to speculate that active RAB-2 facilitates the acceptance of retrograde trafficking carriers at the maturing DCV compartment. This would explain why in unc-108/rab-2 mutants, soluble and transmembrane cargo from maturing DCVs is lost into the endosomallysosomal pathway (Edwards et al, 2009; Sumakovic et al, 2009).

The fact that $C$. elegans GARP mutants are viable indicates that a redundant pathway exists, which compensates for the loss of GARP function during retrograde trafficking of cargo to the Golgi (Luo et al, 2011). In consistent with this, it has been 
suggested that the COG and GARP complexes share overlapping functions (Luo et al, 2011). Therefore, further studies have to be performed to analyze the possible involvement of other multimeric tethering factors, such as COG complex and exocyst in DCV trafficking. This analysis would reveal whether the GARP complex is specifically required during this process.

Double mutant analysis of C. elegans mutants in rund- 1 and vps-51 revealed that the GARP complex and the RAB-2 effector, RUND-1, act in the same pathway. In addition, both proteins RUND-1 and VPS-51 interact in Y2H and co-IP experiments. It is tempting to speculate that RUND-1 might be recycled to the Golgi by the GARP complex. It is unlikely that RUND-1 represents a receptor, since no transmembrane domain was detected for RUND-1. However, by association with membrane-bound proteins, RUND-1 might label vesicles for the retrograde transport by binding to the GARP complex. Further studies should aim to identify RUND-1's exact role in DCV maturation.

Since CCCP-1 possesses three coiled-coil domains, it might form a tethering complex at the Golgi with the GARP complex to accept the incoming retrograde vesicles. In this way, regulated by RAB-2, CCCP-1 might confer specificity for the fusion process by contributing to the tethering of these vesicles to the acceptor membrane. Double mutant analysis and interaction studies would allow a deeper insight into CCCP-1's function.

In addition to RUND-1, we could demonstrate that the effector RIC-19 and the putative RAB-2 specific GAP, TBC-8, also bound to VPS-51 in Y2H and co-IP experiments. However, TBC-8 and the GARP complex seem to act in parallel pathways during DCV maturation, or they have additional functions, independent of the other protein. Unfortunately, the genetic interaction between RIC-19 and VPS-51 could not be tested in this study. Future attempts should be made to study the dependence of these factors. 


\section{XI.2 Specific role of VPS-51 in DCV maturation}

It has been shown that the GARP complex is involved in a broad range of cellular processes such as cell growth, fertility, transport and sorting of cargo proteins [reviewed in (Bonifacino \& Hierro, 2011)]. Loss of GARP function caused various defects in yeast, zebrafish, C. elegans and mammals (Conibear \& Stevens, 2000; Ho et al, 2006; Luo et al, 2011; Perez-Victoria et al, 2008; Perez-Victoria et al, 2010). Interestingly, the loss of Vps51 in yeast had a milder effect on growth compared to the other subunits (Siniossoglou \& Pelham, 2002). A similar phenotype was observed in C. elegans (Luo et al, 2011). Here, vps-51 mutant animals exhibited less severe defects in the brood sizes and in lysosomal morphology compared to mutants in the subunits vps-52-54 (Luo et al, 2011). Therefore, it has been proposed that the subunit VPS-51 might be an auxiliary subunit, whereas the subunits VPS-52/-53/-54 form the core of the GARP complex in yeast and in C. elegans (Conibear \& Stevens, 2000; Luo et al, 2011; Siniossoglou \& Pelham, 2002). However, the phenotypes observed in vps-51 mutants are similar but weaker compared to mutants the other GARP subunits. This demonstrates that the putative auxiliary VPS-51 subunit also contributes to GARP functions, but might have more specialized functions, which were not detected until now (Luo et al, 2011). DCV maturation might represent such a process. In this study, vps-51 mutants displayed the similar decreased NLP-21-derived VENUS fluorescence in the DNC axons like other vps mutants (except vps-54). Furthermore, inactivation of vps-51 had the same impact on the movement impairment like the loss of the remaining subunits. Moreover, VPS-51 interacted with other factors involved in DCV maturation such as TBC-8, RUND-1 and RIC-19. These data indicate that VPS-51 has additional or supporting function during DCV maturation and might be therefore essential for this process. Surprisingly, the VENUS fluorescence levels at the axons in vps-54 mutants were less decreased than those of the remaining vps mutants, although vps-54 mutants exhibited severe defects in other GARP functions (Luo et al, 2011). It has been shown that a missense mutation (L967Q) within mouse Vps54 causes spinal muscular atrophy and defective spermiogenesis summarized as the wobbler mouse phenotype (Boillee et al, 2003; Schmitt-John et al, 2005). The Vps54 (L967Q) still assembles with other GARP subunits 
but leads to a shortened half-life of the complex in vivo resulting in destabilization and reduced levels of the complex (Perez-Victoria et al, 2008). Therefore, it is surprising that mutations in vps-54 caused a milder phenotype in DCV maturation compared to the other subunits, when it might be responsible for stability of the complex. This suggests that VPS-54 might represent an auxiliary subunit of the GARP complex during its function in DCV maturation, whereas VPS-51 might be the main player in this function. Since VPS54 might contribute to DCV maturation by increasing complex stability during GARP function, it is unlikely that different GARP sub-complexes exist in vivo constituting of different assembled subunits.

\section{XI.3 An extended model for DCV maturation}

In this study we could show that the GARP complex exhibited defects in DCV trafficking, indicating that the retrograde transport of cargo from endosomes to the Golgi is essential for proper DCV maturation. Therefore, the existing model of DCV maturation has to be extended (Tooze et al, 2001), in which a retrograde transport of e.g. TGNresident proteins has to be included (Figure XI.1). It is conceiving that important proteins, such as cargo receptors, have to be recycled back to the Golgi-endosomal interface to ensure cycling of the pathway. We speculate that active RAB-2 might facilitate the acceptance of retrograde trafficking carriers at the maturing DCV compartment. Furthermore, we could show that neuronal DCV maturation requires the dynamic exchange and recycling of material within the Golgi-endosomal interface, emphasizing the fact that DCV maturation is a highly-regulated process. 


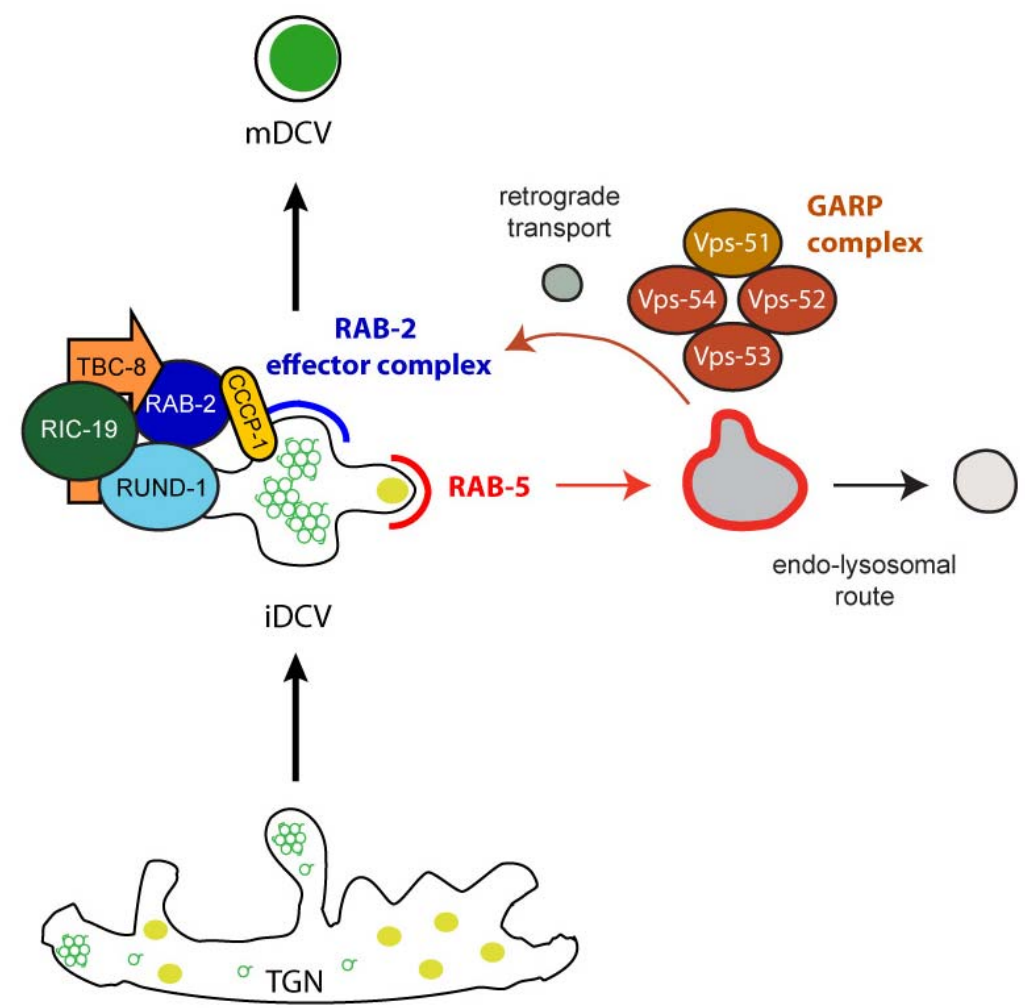

Figure XI.1. Graphic representation of neuronal DCV maturation in C. elegans. Our findings indicate that retrograde transport from endosome to the Golgi is required for DCV maturation. Thus, the existing model has to be extended. The maturing DCV compartment might exhibit different domains on its surface, which are important for specific function ensured by a particular set of proteins (e.g. RAB-2 domains). RAB-2 might be required to accept retrograde cargo vesicles to ensure dynamic remodeling of the maturing DCV. From our previous work, we predict that RAB-5 might form a domain from which mis-sorted cargo is removed to the endolysosomal pathway [our unpublished data; (Sumakovic et al, 2009)]. However, the specifics of this schematic representation are highly speculative, because the molecular mechanisms of interactions have not yet been elucidated. Source: picture modified from (Tooze et al, 2001), with permission from Elsevier. 


\section{Summary and Conclusion}

Despite the importance of dense-core vesicles (DCVs) for the modulation of neurotransmission by the regulated secretion of neuropeptides, hormones and trophic factors, DCV biogenesis in neurons is not well understood. Knowledge was mainly gained from studies in endocrine and neuroendocrine cells, which contain dense-core secretory granules (DCSGs).

Newly synthesized DCVs undergo several maturation steps at the Golgiendosomal interface to obtain mature, exocytosis-competent DCVs. During this maturation process clathrin-mediated remodeling occurs to remove mis-sorted proteins into the endosomal-lysosomal pathway. C. elegans offers a useful model system to study DCV maturation in neurons. Besides the simple nervous system and the possibility to study DCV biogenesis in vivo, the existence of established DCV assays in C. elegans enabled us to get deeper insights into neuronal DCV maturation.

Previously, we have shown that the small GTPase RAB-2 together with its effector, RIC-19, are involved in the retention of cargo within maturing DCVs in C. elegans. In the present study, we identified TBC-8, a neuronally expressed GTPase activating protein (GAP) regulating RAB-2 function during DCV maturation. Furthermore, in addition to RIC-19, we found two novel RAB-2 effectors involved in DCV maturation, such as RUND-1 and CCCP-1. Interestingly, RUND-1 and RIC-19 interacted with the negative regulator of RAB-2, the GAP TBC-8, in binding studies. This suggests a highly-dynamic regulation of RAB-2 during DCV maturation. Our results indicate that active RAB-2 might recruit its own GAP through its effector complex RIC- 
19/TBC-8 to enhance its own deactivation. Thus, this negative feedback loop might represent a novel mechanism to regulate Rab function.

Furthermore, we have shown, for the first time, that retrograde traffic from endosomes to the trans-Golgi is also required for proper DCV maturation. We identified the Golgi associated retrograde tethering complex GARP to cooperate with the RAB-2 effector complex for retention of soluble cargo in maturing DCVs. We propose that active RAB-2 might facilitate the retrieval of endosome-derived vesicles back to the maturing DCV at the post-Golgi compartment. This suggests that neuronal DCV maturation requires the dynamic exchange and recycling of material at the Golgiendosomal interface.

All these findings indicate that DCV maturation is a highly-regulated process and we are just beginning to understand the mechanistic role of all factors involved. 


\section{Materials and Methods}

\section{XIII.1 Material and Reagents}

A list of all chemicals, kits, reagents, enzymes, antibodies, oligonucleotides, plasmids, (E. coli, S. cerevisae, C. elegans) strains, cell lines and laboratory equipments are listed in the Appendix.

\section{XIII.2 Molecular biological methods}

\section{XIII.2.1 Maintenance of $E$. coli}

Liquid bacterial overnight cultures containing the appropriate antibiotic (Appendix) to maintain plasmids were grown to stationary phase at $37^{\circ} \mathrm{C}$ and $225 \mathrm{rpm}$ on an orbital shaker after inoculating a single isolated colony from a freshly grown agar plate into sterile Luria-Bertani (LB) medium (Appendix).

\section{XIII.2.2 Preparation of chemical competent $E$. coli}

An overnight culture of $E$. coli was subcultured 1:100 in LB media and incubated at $37^{\circ} \mathrm{C}$ with shaking at $220 \mathrm{rpm}$ until an $\mathrm{OD}_{600}$ of 0.5 was reached. The culture flask was chilled on ice for $20 \mathrm{~min}$ and cells were collected by centrifugation at $5000 \mathrm{rpm}$ for $15 \mathrm{~min}$ at $4{ }^{\circ} \mathrm{C}$. Cells were resuspended in $10 \mathrm{ml}$ ice-cold TSS solution (Appendix) and transferred as $100 \mu \mathrm{l}$ aliquots into $1.5 \mathrm{ml}$ Eppendorf tubes. These tubes were snap-frozen in liquid nitrogen and stored at $-80^{\circ} \mathrm{C}$. Protocol was taken from (Chung et al, 1989). 


\section{XIII.2.3 Molecular cloning}

In this study, molecular cloning was performed by using classical molecular biology techniques (Sambrook and Russel, 2001). List of all materials, equipments and reagents are shown in the Appendix.

\section{XIII.2.3.1 Polymerase Chain Reaction (PCR)}

PCR was conducted for the amplification of different genes (Appendix) using a cDNA library (ProQuest, Invitrogen) with specific primers (Appendix) and cloned into different vectors (Appendix). Genomic promoter loci was PCR amplified using purified C. elegans genomic DNA (provided by Dr. Nikhil Sasidharan). For cloning of PCR fragments the PfuUltra II Polymerase (Stratagene) was used. In general the

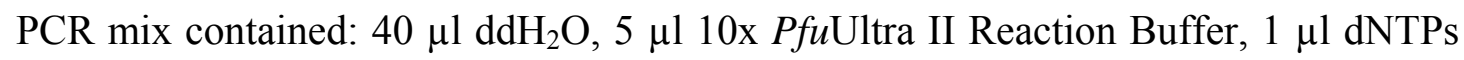
[12.5 mmol], $1 \mu 1$ forward primer $[10 \mu \mathrm{M}], 1 \mu 1$ reverse primer $[10 \mu \mathrm{M}], 1 \mu 1$ PfuUltra II Polymerase and $1 \mu 1$ template DNA ( $20 \mathrm{ng})$. PCR reactions were placed into a thermocycler (eppendorf) and run with following general parameters: $95^{\circ} \mathrm{C} 1 \mathrm{~min}$; $35 \mathrm{x}\left[95^{\circ} \mathrm{C} 20 \mathrm{sec} \rightarrow \mathrm{x}^{\circ} \mathrm{C} 20 \mathrm{sec} \rightarrow 72^{\circ} \mathrm{C} \mathrm{x} \mathrm{min}\right] ; 72^{\circ} \mathrm{C} 3 \mathrm{~min} ; 4^{\circ} \mathrm{C}$. To mutate the catalytic arginine of TBC-8 a site-directed mutagenesis approach was utilized. PCR products were analysed on 1\% agarose gels (section XIII.2.3.3). Positive DNA clones were verified by sequencing reaction (Qiagen).

\section{XIII.2.3.2 Digestion of DNA}

The backbone plasmids and the amplified DNA inserts were digested using the appropriate restriction enzymes (Appendix). In general, the $20 \mu 1$ reaction digest contained $1 \mu \mathrm{g}$ vector or insert DNA, $2 \mu \mathrm{l} 10 \mathrm{x}$ Buffer and $1 \mu \mathrm{l}$ of appropriate restriction enzymes. This mixture was incubated for $2-3$ hours at $37^{\circ} \mathrm{C}$. DNA was analysed on a $1 \%$ agarose gel (section XIII.2.3.3). Digestion was also used to find clones harbouring the correct plasmids after cloning.

\section{XIII.2.3.3 Agarose gel electrophoresis}

The success of PCR reactions and the size of restriction products were verified using agarose gel electrophoresis. Standard melting point agarose with 1x TBE buffer (Appendix) and $0.1 \mu \mathrm{l} / \mathrm{ml}$ ethidium bromide were used to prepare $1 \%$ agarose gels. Samples were mixed with 5x DNA loading buffer (Appendix) and run 30-40 min in 
1x TBE buffer at $140 \mathrm{~V}$ in a horizontal electrophoresis system unit. Desired DNA was cut out and extracted using commercial Gel Extraction Kits.

\section{XIII.2.3.4 Ligation}

Ligations of backbone plasmids with amplified DNA inserts were performed by mixing the following reagents: $14 \mu 1$ digested insert DNA, $4 \mu 1$ digested vector, 2 $\mu 1$ 10x ligation buffer containing $10 \mathrm{mM}$ ATP and $1 \mu 1$ ligase [5 U/ $\mu 1]$. Reaction mixes were incubated either overnight at $15^{\circ} \mathrm{C}$ or 3 hours at room temperature (RT) and transformed into E. coli DH5a (section XIII.2.3.5).

\section{XIII.2.3.5 Chemical transformation of $E$. coli}

One hundred microlitres of chemical transformation competent E. coli DH5 $\alpha$ were thawed on ice and placed into $1.5 \mathrm{ml}$ Eppendorf tubes. After the DNA was added and incubated on ice for $20 \mathrm{~min}$, the mixture was heat shocked for $2 \mathrm{~min}$ at $42^{\circ} \mathrm{C}$ and incubated for $2 \mathrm{~min}$ on ice. Nine hundred microlitre of LB medium was added, incubated for 1 hour with shaking at $225 \mathrm{rpm}$ at $37^{\circ} \mathrm{C}$ and plated on $\mathrm{LB}$ medium plates containing the appropriate antibiotic. The plates were then incubated at $37^{\circ} \mathrm{C}$ overnight.

DNA of promising bacterial colonies was purified by using a commercial MiniPrep Kit and control digested (section XIII.2.3.2). The product sizes were checked by agarose gel electrophoresis (section XIII.2.3.3). Finally, the sequences were verified by sequencing (Qiagen, Hilden). In order to purify high amounts of plasmid DNA, a commercial MidiPrep Kit (Appendix) was used.

\section{XIII.3 Protein biochemical methods}

\section{XIII.3.1 Expression and solubility test of recombinant proteins}

To test the expression and solubility of GST-tagged proteins TBC-8 full length and different fragments of TBC-8 (Table XIII.1) were transformed into E. coli BL21CodonPlus(DE3)-RIL and examined as follows. Overnight cultures were subcultured 1:50 into sterile flasks containing LB medium with the appropriate antibiotic, grown to an $\mathrm{OD}_{600}$ of 0.4 and were induced with a final concentration of $50 \mu \mathrm{M}$ isopropylbeta-D-thio-galactopyranoside (IPTG) overnight at $37^{\circ} \mathrm{C}$. The bacteria were harvested 
by centrifugation at $4000 \mathrm{rpm}$ for $10 \mathrm{~min}$ (Eppendorf $5810 \mathrm{R}$, rotor A-4-62). The pellet was resuspended in cleavage buffer (Appendix) and cells were disrupted by several rounds of sonication on ice ( $60 \%$ amplitude, 0.5 cycle). Cell debris and other insoluble material were removed by centrifugation at $10.000 \mathrm{rpm}$ (Sorval RC6, rotor SS-34) for $30 \mathrm{~min}$ at $4^{\circ} \mathrm{C}$. Both supernatant and pellet fractions were solubilised by boiling 5-10 $\mathrm{min}$ in 4x SDS sample buffer (Appendix) and subjected to SDS-10\% polyacrylamide gels using 1x SDS running buffer (Appendix) at $160 \mathrm{~V}$ for $60-90 \mathrm{~min}$ to verify whether GST-tagged TBC-8 full length and fragments were soluble. Gels were stained with Coomassie Brilliant Blue R250 stain (Appendix) by emersion in stain for $30 \mathrm{~min}$ at RT on a rocking platform. Gels were then destained by Coomassie destaining buffer (Appendix) until bands appeared.

Table XIII.1: Different constructs used for expression and solubility test of recombinant TBC-8

\begin{tabular}{|c|c|c|c|}
\hline Construct & $\begin{array}{c}\text { Amplified with } \\
\text { primers }\end{array}$ & fusion tag & $\begin{array}{c}\text { Predicted } \\
\text { GST-TBC-8 } \\
\text { size }\end{array}$ \\
\hline $\begin{array}{c}\text { pGST-tbc-8 full length } \\
\text { TBC-8 fragment: 1-903 aa }\end{array}$ & oGQ1622/oGQ1752 & GST & $\sim 130 \mathrm{kDa}$ \\
\hline $\begin{array}{c}\text { pGST-tbc-8 large } \\
\text { TBC-8 fragment: 329-903 aa }\end{array}$ & oGQ1801/oGQ1752 & GST & $\sim 94 \mathrm{kDa}$ \\
\hline $\begin{array}{c}\text { pGST-tbc-8 medium } \\
\text { TBC-8 fragment: 451-903 aa }\end{array}$ & oGQ1802/oGQ1752 & GST & $\sim 80 \mathrm{kDa}$ \\
\hline $\begin{array}{c}\text { pGST-tbc-8 small } \\
\text { TBC-8 fragment: } 587-903 \text { aa }\end{array}$ & oGQ1792/oGQ1752 & GST & $\sim 64 \mathrm{kDa}$ \\
\hline
\end{tabular}

\section{XIII.3.2 SDS PAGE and Western blot analysis}

Prepared samples were boiled for $5 \mathrm{~min}$ and run on SDS-polyacrylamide gels (Appendix) using a BioRad Mini-PROTEAN ${ }^{\circledR} 3$ Cell electrophoresis system and $1 \mathrm{x}$ SDS running buffer (Appendix) at $160 \mathrm{~V}$ for 60-90 min. Protein ladder (PageRuler ${ }^{\mathrm{TM}}$ Prestained Protein Ladder, Fermentas) was used as a marker. The proteins were transferred to a nitrocellulose membrane (Protran ${ }^{\circledR}$ Whatman ${ }^{\circledR}$ ) following standard protocols using 1x transfer buffer (Appendix) at $100 \mathrm{~mA}$ for $60 \mathrm{~min}$ in a semidryblotting device. Membranes were then blocked in Tris-buffered saline (TBS) (Appendix) containing 0.1\% (v/v) Tween $20(\mathrm{TBST})$ and 3\% (w/v) powdered fat-free skim milk for $30 \mathrm{~min}$ at RT and probed with the primary antibodies (Appendix) overnight at $4^{\circ} \mathrm{C}$. After washing $3 \times 10$ min with 1 x TBST, 1:10,000 diluted goat anti- 
mouse or anti-rabbit, respectively, horseradish peroxidase (HRP)-conjugated secondary antibody (Jacksons Laboratory, Bar Harbor, Me) was incubated for 45 min at RT. After washing with TBST as above, antibody complexes were detected using a mixture of luminol, parahydroxycoumarinacid and $\mathrm{H}_{2} \mathrm{O}_{2}$ (Appendix) and exposing the membrane for different lengths of time depending on the signal strength. A FujiFilm LAS 3000 processor was used to develop images which were edited using the ImageJ software (National Institutes of Health).

\section{XIII.4 C. elegans based methods}

\section{XIII.4.1 Maintenance of C. elegans strains}

C. elegans strains were maintained on nematode growth medium (NGM) agar plates seeded with E. coli OP50 bacteria and kept at $20^{\circ} \mathrm{C}$ (Brenner, 1974). General protocols of culturing worms, e.g. general maintenance instructions, NGM plates and seeding preparations, freezing and recovering of $C$. elegans stocks are described in wormbook (http://wormbook.org/). Mutant strains were obtained from the C. elegans Gene Knockout Consortium (CGC-Vancouver, Canada and Minnesota/Oklahoma, USA) and the National Bioresource Project for the Nematode C. elegans (Tokyo, Japan). All C. elegans strains used in this study are listed in Appendix. Descriptions of respective strains are listed in wormbase (www.wormbase.org).

\section{XIII.4.2 Freezing and recovery of $C$. elegans stocks}

C. elegans strains of six freshly starved $10 \mathrm{~cm}$ plates containing mainly L1-L2 larvae stages were washed with $\mathrm{S}$ buffer (Appendix) for $5 \mathrm{~min}$ at $800 \mathrm{rpm}$ at RT. Supernatant was removed and $3 \mathrm{ml}$ of worm suspension was mixed with the equal volume of freezing solution and aliquoted into 6 cryotubes, each filled with one millilitre worm solution. These tubes were placed into a Styrofoam box and slowly frozen at $-80^{\circ} \mathrm{C}$. After 24 hours, one cryotube was used to test whether the freezing was successful and the worms were able to recover.

For recovery of $C$. elegans strains, a frozen aliquot was thawed at RT and transferred onto a fresh NGM plate. On the next day, the plate was inspected for recovered worms and if required, those were transferred onto a fresh NGM plate. 


\section{XIII.4.3 Synchronization or decontamination of C. elegans (egg preparation)}

Worms grown on a $10 \mathrm{~cm}$ plate were washed in M9 buffer (Appendix) and concentrated to $3.5 \mathrm{ml}$. To this worm solution, $500 \mu \mathrm{l} 5 \mathrm{M} \mathrm{NaOH}$ and $1 \mathrm{ml}$ bleach solution (5\%) were added. Worms were resolved by vortexing for circa $10 \mathrm{~min}$. During vortexing, the success of egg release was assessed by light microscopy. This worm solution was centrifuged for $0.5 \mathrm{~min}$ at $1300 \mathrm{~g}$ and washed with $10 \mathrm{ml} \mathrm{H}_{2} \mathrm{O}$. The supernatant was removed and eggs were placed onto a fresh NGM plate.

\section{XIII.4.4 Crossing of C. elegans}

To cross deletion mutants of interest [e.g. tbc-8(tm3802)] into stable integration lines [e.g. nuIs183] following procedure was used: First males of the integrated line was generated by placing 3 to 4 hermaphrodites together with 10-15 males of ccIs4251, him-8 onto a new NGM plate for 3-5 days at $20^{\circ} \mathrm{C}$. From this plate 10-15 male F1 worms were picked that showed both markers, pmyo-3::mt-gfp; pmyo3::nls-gfp [ccIs4251; (Fire et al, 1998)] and pmyo-2::gfp [nuIs183; (Sieburth et al, 2007)] (confirming success of the cross) and were placed together with 3-4 hermaphrodites of the deletion strain [e.g. $t b c-8(t m 3802)]$. F1 progeny were singled onto new NGM plates and allowed to lay eggs. [When double mutants were generated, F1 progeny was tested for heterozygosity of both alleles by single worm PCR (section XIII.4.6)]. F2 progeny was selected for corresponding markers and singled again on NGM plates to lay eggs. By using single worm PCR (section XIII.4.6), F2 progeny was tested for homozygosity of the deletion allele and the presence of fluorescence markers was analysed by microscopy.

\section{XIII.4.5 Generation of transgenic C. elegans worm lines via microinjection}

For expression pattern analysis and co-localization studies of proteins of interest with different intracellular markers, transgenic worm strains carrying extrachromosomal arrays were created via microinjection. Young adult hermaphrodites were immobilized under oil on a pad of dried 2\%-agarose. Microinjection of DNA mixtures (Appendix) into the distal gonads was performed using a micromanipulator and a pump as described earlier (Mello \& Fire, 1995). After injection worms were rescued with M9 buffer (Appendix) and placed onto a new 
NGM plate seeded with E. coli OP50. Different co-injection markers were used to analyse successful transformation of C. elegans: the dominant mutant rol-6(su1006) allele of the collagen gene rol-6 (Kramer et al, 1990) which causes the so-called "roller phenotype" because the worms move in circles and rotate around their body axis or the fluorescence marker ttx-3::gfp which labels two head neurons, AIY left and right interneurons, in C. elegans (Hobert et al, 1997). These co-injections markers enable the identification of positive F1 progeny which were singled to identify positive F2 progeny carrying the extrachromosomal array with a good transmission rate.

\section{XIII.4.6 Single worm PCR / Worm PCR}

Worm PCR was used to test heterozygosis of deletion strains. At first, worms were lysed in lysis buffer (Appendix) by freezing worms for at least $10 \mathrm{~min}$ at $-80^{\circ} \mathrm{C}$ and incubating them at $65^{\circ} \mathrm{C}$ for 1 hour followed by a $95^{\circ} \mathrm{C}$ step for 20 min to inactivate proteinase $\mathrm{K}$. The $25 \mu \mathrm{l}$ PCR mix contained $19.25 \mu 1 \mathrm{ddH}_{2} \mathrm{O}, 2.5 \mu 110 \mathrm{x}$ worm PCR Reaction Buffer (Appendix), $0.5 \mu \mathrm{l}$ dNTPs [12.5 mmol], $0.25 \mu 1$ forward primer $[10 \mu \mathrm{M}], 0.25 \mu 1$ reverse primer $[10 \mu \mathrm{M}]$ (Appendix), $0.25 \mu 1$ Tag Polymerase and $2 \mu \mathrm{l}$ template DNA (worm lysis). The reaction was placed into a thermocycler and run with following parameters: $94^{\circ} \mathrm{C} 4 \mathrm{~min} ; 35 \mathrm{x}\left[94^{\circ} \mathrm{C} 15 \mathrm{sec} \rightarrow \mathrm{x}^{\circ} \mathrm{C} 30 \mathrm{sec} \rightarrow\right.$ $72^{\circ} \mathrm{C} \mathrm{x} \mathrm{sec}$; $72^{\circ} \mathrm{C} 5 \mathrm{~min} ; 4^{\circ} \mathrm{C}$. PCR products were analysed on $1 \%$ agarose gels (section XIII.2.3.3).

\section{XIII.4.7 Movement assay}

To analyse the locomotion behaviour of C. elegans strains, worms were transferred without any E. coli OP50 traces to a non-seeded NGM plate. After an initial adaptation period of $30 \mathrm{~min}$, the body bends of every worm were counted for 3 min. Ten to thirty non-starved young adult worms per strain were analysed, and the total body bends (corresponding to a whole $360^{\circ}$ sine wave) were divided by three to obtain the body bends per min.

\section{XIII.4.8 Co-immunoprecipitation of proteins from C. elegans extracts}

Two big plates with mixed staged worms were harvested and washed once with M9 buffer (Appendix). Supernatant was removed and $200 \mu 1$ M9 buffer mixed with worms were transferred to an eppendorf tube and snap-frozen in liquid nitrogen 
before storing the tube in $-80^{\circ} \mathrm{C}$ freezer. The frozen worm pellet was grounded with a mortar and pestle in the presence of liquid nitrogen to keep the protein extract lower than $4^{\circ} \mathrm{C}$. While thawing about $1 \mathrm{ml}$ homogenization buffer (Appendix) was added. After well-grounding the worms, the protein extract was transferred to an eppendorf tube and centrifuged for $10 \mathrm{~min}$ at $500 \mathrm{~g}$ at $4^{\circ} \mathrm{C}$ to remove cell debris. From here, 100 $\mu 1$ "input" sample were taken and mixed with $25 \mu 14 x$ Laemmli buffer (Appendix). The supernatant was then incubated with $4 \mu \mathrm{g}$ antibodies (Appendix) for at least 3 hours at $4{ }^{\circ} \mathrm{C}$. Thirty $\mu 1$ of equilibrated protein G-sepharose were added and incubated for 2-3 hours at $4^{\circ} \mathrm{C}$. Beads were washed 3 times with $1 \mathrm{ml}$ cooled homogenization buffer and centrifuged for $0.5 \mathrm{~min}$ at $10.000 \mathrm{rpm}$ at $4^{\circ} \mathrm{C}$. Supernatant was removed and protein complexes were eluted from the beads by adding 1x Laemmli buffer (Appendix). Prepared samples were subjected to Western blot analysis (section XIII.3.2).

\section{XIII.4.9 RNA interference (RNAi) by feeding}

RNAi by feeding was performed essentially as described in (Kamath et al, 2001). Briefly, HT115 (DE3) bacteria were transformed with L4440 feeding vector [L4440 empty vector (negative control) or L4440-gene-of-interest, respectively] (Figure XIII.1) (Appendix) and cultured on LB plates with $100 \mu \mathrm{g} / \mathrm{ml}$ ampicillin. A single colony was inoculated into $20 \mathrm{ml} \mathrm{LB}$ with ampicillin and grown overnight. These cultures were used for seeding NGM plates containing $100 \mu \mathrm{g} / \mathrm{ml}$ ampicillin and $1 \mathrm{mM}$ IPTG.

Ten L4 eri-1(mg366), nuIs183 hermaphrodites were put on each plate (seeded with the E. coli HT115 strain containing the respective L4440 vector) to allow egg laying for about 12 hours and were transferred onto a new RNAi plate. Usually, the progeny (in their young adult worm stage) of the third plate was used to analyse the VENUS-fluorescence level in the dorsal nerve cord by confocal microscopy (section XIII.4.10). Fluorescence of NLP-21-derived VENUS in the dorsal nerve cord was normalized to the VENUS fluorescence of mock RNAi (L4440) worms. 


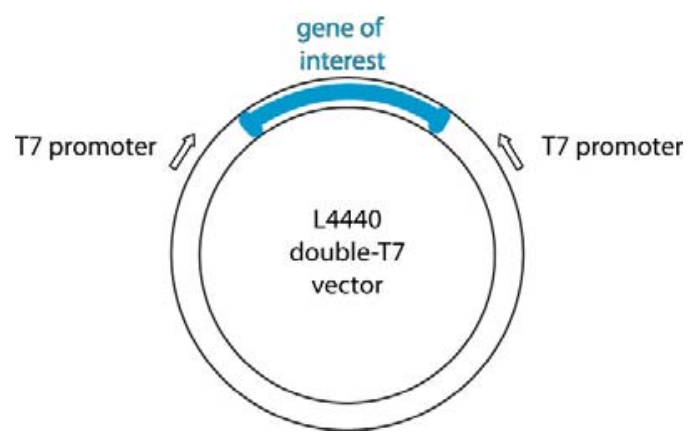

Figure XIII.1. RNAi feeding vector L4440. The vector L4440 harbouring the gene of interest were transformed into E. coli HT115. These cultures were used for seeding NGM plates containing IPTG to induce expression of T7 polymerase that is encoded by HT115 to produce double-stranded RNA of the gene of interest.

\section{XIII.4.10 Microscopic analysis of $C$. elegans}

For confocal microscopy, live worms were paralyzed with $50 \mathrm{mM} \mathrm{NaN}_{3}$ on 2\% agarose pads. An inverted Confocal Laser Scanning Microscope (SP2, Leica) with a $100 \mathrm{x}$ oil objective $(\mathrm{NA}=1.4)$ (co-localization studies) or a $63 \mathrm{x}$ oil objective $(\mathrm{NA}=$ 1.32) (DCV assay, expression pattern studies) was used. GFP was excited with a laser at $488 \mathrm{~nm}$, YFP at $514 \mathrm{~nm}$ and tagRFP as well as mCherry at $561 \mathrm{~nm}$. The scan was performed with a resolution of $1024 \times 1024$ pixels, and the pinhole was set to 1 airy unit. For co-localization studies, the scan was performed sequentially to prevent excitation of RFP through the GFP or YFP excitation light. Here, images of neuronal cell bodies from the ventral nerve cord were taken. Image stacks were captured and average intensity projections were obtained using the Leica software. These images were then edited using ImageJ software (National Institutes of Health).

Furthermore, images of expression pattern were taken using a Perkin Elmer Spinning Disc Confocal Microscope. These images were edited using Adobe Photoshop $^{\circledR}$ software.

For quantification studies from DNC, neuronal cell bodies and coelomocytes (DCV assay, imaging of endocytosed ssGFP in coelomocytes secreted from muscle cells), young adult worms were imaged as described previously (Sieburth et al, 2007). For cell body and DNC imaging, the neuronal cell bodies and DNC were oriented toward the objective, whereas for coelomocytes imaging, the posterior coelomocytes were oriented laterally. Image stacks of the regions of interest were captured and maximum intensity projections were obtained using the Leica software. For all 
obtained images, the same settings were used. These projections were thresholded and quantified using the ImageJ software (National Institutes of Health). All these data were normalized to wild type.

For quantification of vesicle sizes in neuronal cell bodies in nuIs183 background strains, obtained images of cell bodies (explained above) were analyzed using the ImageJ software (National Institutes of Health).

In order to image apoptotic cell corpses, image stacks of the distal gonad arms were captured and projections were obtained using the Perkin Elmer Spinning Disc Confocal Microscope. From these pictures the number of cell corpses in the distal gonad arm and in the gonad loop was counted manually for each strain.

\section{XIII.4.11 High pressure freezing / Freeze substitution / Electron microscopy}

A $100 \mu \mathrm{m}$ deep aluminum platelet (Microscopy Services, Flintbek) was filled with E. coli OP50 suspension. About 20 young adult worms were transferred into the chamber and immediately frozen using a BalTec HPM 10. Freeze substitution was carried out in a Leica AFS2. Incubations were performed at $-90^{\circ} \mathrm{C}$ for $100 \mathrm{~h}$ in $0.1 \%$ tannic acid, $7 \mathrm{~h}$ in $2 \% \mathrm{OsO}_{4}$, and at $-20^{\circ} \mathrm{C}$ for $16 \mathrm{~h}$ in $2 \% \mathrm{OsO}_{4}$, followed by embedding in EPON at RT (Rostaing et al, 2004) (all solutions w/v in dry acetone). Fifty nanometer sections were mounted on copper slot grids and placed for $10 \mathrm{~min}$ on drops of $4 \%(\mathrm{w} / \mathrm{v})$ uranyl acetate in $75 \%$ methanol and then washed in distilled water. After air drying, the grids were placed on lead citrate (Reynolds, 1963) for $2 \mathrm{~min}$ in a $\mathrm{CO}_{2}$-free chamber, and rinsed in distilled water. Micrographs were taken with a 1024 $\times 1024$ CCD detector (Proscan CCD HSS 512/1024; Proscan Electronic Systems, Scheuring, Germany) in a Zeiss EM 902A, operated in the bright field mode. The SV and DCV diameter and distribution at the synapse of motoneurons were analyzed by a semi-automated analysis software XtraCount (manuscript in preparation).

For the analysis of presynaptic terminals and SV and DCV distributions, cross sections of young adult animals were used to image cholinergic neuro-muscular junction (NMJ) synapses in the ventral nerve cord, posterior to the nerve ring. These cholinergic NMJ synapses were defined as polyadic synapses projecting onto muscle arms as well as other neurons according to the standard convention in the C. elegans EM field. Axons showing a clearly visible presynaptic density and synaptic vesicles 
were defined as synapse. The mean area of presynaptic terminal was measured in cross sections using the surrounding axonal membrane as border.

For the morphological analysis of neurons, ten motoneuronal cell bodies localized in the ventral nerve cord in wild type worms were compared with seven cell bodies in tbc-8(tm3802) worms.

\section{XIII.4.12 Texas red-conjugated BSA endocytosis assay}

For the analysis of postendocytic trafficking within coelomocytes of the fluidphase endocytosis marker TR-BSA, the integrated strain bIs34[prme-8::rme-8-GFP] was crossed into $t b c-8(t m 3802)$ to label RME- 8 positive endosomes. TR-BSA (1 $\mathrm{mg} / \mathrm{ml}$ ) was injected into the body cavity in the pharyngeal region of young adult worms as described previously (Zhang et al, 2001). Uptake and postendocytosis was analyzed after 10, 30 and 50 min after injection by confocal microscopy. At least five animals were injected for each time point. Single fluorescence images at the middle plane of each coelomocyte were taken and line-averaged. Images were edited using ImageJ software (National Institutes of Health).

\section{XIII.4.13 ssGFP endocytosis assay}

To analyze the kinetics of endocytosis and degradation of ssGFP, the strain arIs36[phsp::ssGFP] (Fares \& Greenwald, 2001) was crossed into tbc-8(tm3802). Young adult worms were grown at $20^{\circ} \mathrm{C}$ before a heat-shock at $33^{\circ} \mathrm{C}$ for 30 min was performed. Afterwards, worms were placed back to $20^{\circ} \mathrm{C}$ to recover until they were used for imaging. The uptake of ssGFP into coelomocytes and degradation of endocytosed GFP was monitored after 3.5, 6 and 28 hours after heat-shock. All fluorescence pictures were taken with the same settings. At the time points where fluorescence was hard to detect during imaging, Differential Interference Contrast (DIC) Microscopy images of the respective specimen were taken. Later, these images were used to outline the cell boundaries of coelomocytes. Images were edited using the ImageJ software (National Institutes of Health).

To analyze the steady-state endocytosis of ssGFP within coelomocytes, the strain arIs37[pmyo3::ssGFP] (Fares \& Greenwald, 2001) was crossed into tbc8(tm3802). Endocytosed GFP was analyzed by confocal microscopy described in section XIII.4.10. 


\section{XIII.5 Tissue culture based methods}

\section{XIII.5.1 Maintenance of cell lines}

HEK293 cells were grown in high glucose (4.5 g/l) DMEM supplemented with $10 \% \mathrm{FBS}, 110 \mathrm{mg} / 1$ sodium pyruvate, $2 \mathrm{mM}$ glutamine, $100 \mathrm{U} / \mathrm{ml}$ penicillin, and 10 $\mu \mathrm{g} / \mathrm{ml}$ streptomycin in a $5 \% \mathrm{CO}_{2}$ incubator at $37^{\circ} \mathrm{C}$. When reaching a confluence of about $90 \%$, cells were split by washing them once in $8 \mathrm{ml}$ PBS and incubating them for 5-10 min in $4 \mathrm{ml}$ Trypsin-EDTA (1:20 dilution in PBS). After stopping the trypsin digestion with the addition of media, the cells were appropriately diluted for further maintenance or transfection.

\section{XIII.5.2 Transfection of cell lines}

For co-immunoprecipitation, $1.6 \times 10^{6}$ HEK293 cells were plated onto $10 \mathrm{~cm}$ Petri dishes 24 hours before transfection, which was performed using TurboFect $^{\mathrm{TM}}$ in vitro Transfection Reagent according to the manufacturer's protocol (Fermentas). Per transfection two $10 \mathrm{~cm}$ Petri dishes were prepared. For each Petri dish the following transfection mixture was prepared: $1.6 \mathrm{ml}$ serum-free media, $8 \mu \mathrm{g}$ of each plasmid, 24 $\mu 1$ TurboFect $^{\mathrm{TM}}$ in vitro Transfection Reagent. Mix immediately by vortexing and incubate the mixture for 15-20 $\mathrm{min}$ at RT. HEK293 cells were taken out of the incubator and were not subjected to any further washing or other treatment before the transfection suspension was slowly dropped onto the cells. Petri dishes were carefully shaken to evenly distribute the transfection mixture within the media and placed back into the incubator for 24 hours at $37^{\circ} \mathrm{C}$.

\section{XIII.5.3 Co-immunoprecipitation of proteins from transfected HEK293 cells}

Twenty four hours after transfection HEK293 cells were washed with PBS and harvested in lysis buffer (Appendix) for $30 \mathrm{~min}$ at $4^{\circ} \mathrm{C}$. Lysates were pre-cleared by centrifugation at $4{ }^{\circ} \mathrm{C}$ before supernatant was incubated with $2 \mu \mathrm{g}$ monoclonal antiGFP antibody (clone 3E6, invitrogen) for $3 \mathrm{~h}$ at $4^{\circ} \mathrm{C}$. Protein $\mathrm{G}$ Plus-sepharose (Pierce) beads were added. After an another incubation time of 2 hours, the beads were washed three times with washing buffer (Appendix) and resuspended in Laemmli loading buffer. Prepared samples were subjected to Western blot analysis to detect co-precipitated proteins (section XIII.3.2). 


\section{XIII.6 Yeast based methods}

\section{XIII.6.1 Maintenance of yeast strains}

Liquid yeast overnight cultures were grown to stationary phase at $30^{\circ} \mathrm{C}$ and $180 \mathrm{rpm}$ on an orbital shaker (New Brunswick Scientific, Innova40) after inoculating a single isolated colony from an agar plate into sterile yeast full media (YAPD) (Appendix).

\section{XIII.6.2 Yeast tansformation}

Short transformation protocol

Seven hundred microlitres of a yeast overnight culture (section XIII.6.1) were spun down for $2 \mathrm{~min}$ at $2000 \mathrm{rpm}$ in a bench centrifuge. The supernatant was removed and the pellet was resuspended with $100 \mu$ l One-Step-Buffer (Appendix). Two $\mu \mathrm{g}$ of each plasmid was added and mixed by vortexing. This mixture was incubated for 15 min at RT. A heat-shock for $30 \mathrm{~min}$ at $45^{\circ} \mathrm{C}$ followed before the suspension was plated onto selection plates with glass beads. Plates were incubated at $30^{\circ} \mathrm{C}$ for $3-4$ days.

\section{Long transformation protocol}

An overnight yeast culture was subcultured 1:100 to an $\mathrm{OD}_{600}$ of 0.1 and incubated at $30^{\circ} \mathrm{C}$ in a shaker until an $\mathrm{OD}_{600}$ of 0.8 was reached. One millilitre culture was used per transformation reaction. Yeast was pelleted for $2 \mathrm{~min}$ at $8000 \mathrm{rpm}$ and cells were washed in $1 \mathrm{ml} 100 \mathrm{mM}$ lithium acetate before resuspended in $240 \mu 150 \%$ PEG 3350 (Appendix), $36 \mu \mathrm{l} 1 \mathrm{M}$ lithium acetate, $10 \mu \mathrm{l}$ carrier DNA $(10 \mathrm{mg} / \mathrm{ml}$ salmon sperm DNA), $1 \mu \mathrm{g}$ of each plasmid DNA and $70 \mu \mathrm{H}_{2} \mathrm{O}$. After a heat-shock at $42^{\circ} \mathrm{C}$ for $40 \mathrm{~min}$, the yeast/DNA suspension was directly centrifuged for $2 \mathrm{~min}$ at $8000 \mathrm{rpm}$ and washed in $1 \mathrm{ml} \mathrm{H}_{2} \mathrm{O}$. The cells were resuspended in $1 \mathrm{ml}$ YAPD media (Appendix) and recovered for 1 hour at $30^{\circ} \mathrm{C}$ in a shaker. Subsequently, the cells were washed again in $1 \mathrm{ml} \mathrm{H}_{2} \mathrm{O}$ and plated with glass beads onto yeast selection plates.

\section{XIII.6.3 Yeast-Two Hybrid system}

The Matchmaker yeast two-hybrid assay was performed according to the manufacturer's protocol (Clontech). The appropriate plasmid (Appendix) combinations were transformed (section XIII.6.2) into the yeast strain AH109 
(Clontech) and spread onto selective growth media lacking leucine and tryptophane for plasmid selection (Appendix). Protein interactions were tested as follows: several clones of transformants were mixed and diluted to $\mathrm{OD}_{600}$ of 0.4 . Five microlitre of this yeast dilution was spotted onto selective plates lacking leucine, tryptophan and histidine. Interactions were identified by growth after 3-4 days. All interacting proteins were tested for self-activation as described above using the appropriate empty vector pGBKT7 or pGADT7, respectively.

\section{XIII.7 Bioinformatics}

The molecular weight and the isoelectric point of proteins were calculated using the expasy program (http://web.expasy.org/compute_pi/). Transmembrane domain predictions were done using the TMHMM program (http://www.cbs.dtu.dk/services/TMHMM-2.0/). Scans for known protein domains were performed using the SMART program (http://smart.embl-heidelberg.de/)

In order to find orthologs of TBC-8 sequence comparisons in non-redundant databases was carried out using the Blastp algorithm (http://www.ncbi.nlm.nih.gov/BLAST/). Sequence alignments were performed with the MUSCLE program (Edgar, 2004) (http://www.ebi.ac.uk/Tools/msa/muscle/) using default parameters and exhibited with Boxshade 3.2.1 (http://www.ch.embnet.org/software/BOX_form.html). 


\section{Bibliography}

Aivazian D, Serrano RL, Pfeffer S (2006) TIP47 is a key effector for Rab9 localization. $J$ Cell Biol 173: 917-926

Albert S, Gallwitz D (1999) Two new members of a family of Ypt/Rab GTPase activating proteins. Promiscuity of substrate recognition. J Biol Chem 274: 33186-33189

Albert S, Will E, Gallwitz D (1999) Identification of the catalytic domains and their functionally critical arginine residues of two yeast GTPase-activating proteins specific for Ypt/Rab transport GTPases. EMBO J 18: 5216-5225

Aligianis IA, Johnson CA, Gissen P, Chen D, Hampshire D, Hoffmann K, Maina EN, Morgan NV, Tee L, Morton J, Ainsworth JR, Horn D, Rosser E, Cole TR, Stolte-Dijkstra I, Fieggen K, Clayton-Smith J, Megarbane A, Shield JP, Newbury-Ecob R, Dobyns WB, Graham JM, Jr., Kjaer KW, Warburg M, Bond J, Trembath RC, Harris LW, Takai Y, Mundlos S, Tannahill D, Woods CG, Maher ER (2005) Mutations of the catalytic subunit of RAB3GAP cause Warburg Micro syndrome. Nat Genet 37: 221-223

Aligianis IA, Morgan NV, Mione M, Johnson CA, Rosser E, Hennekam RC, Adams G, Trembath RC, Pilz DT, Stoodley N, Moore AT, Wilson S, Maher ER (2006) Mutation in Rab3 GTPase-activating protein (RAB3GAP) noncatalytic subunit in a kindred with Martsolf syndrome. Am J Hum Genet 78: 702-707

Anelli T, Sitia R (2008) Protein quality control in the early secretory pathway. EMBO $J$ 27: 315-327

Artalejo CR, Elhamdani A, Palfrey HC (1998) Secretion: dense-core vesicles can kissand-run too. Curr Biol 8: R62-65

Arvan P, Castle D (1998) Sorting and storage during secretory granule biogenesis: looking backward and looking forward. Biochem J 332 ( Pt 3): 593-610

Bahadoran P, Aberdam E, Mantoux F, Busca R, Bille K, Yalman N, de Saint-Basile G, Casaroli-Marano R, Ortonne JP, Ballotti R (2001) Rab27a: A key to melanosome transport in human melanocytes. J Cell Biol 152: 843-850 
Balkowiec A, Katz DM (2000) Activity-dependent release of endogenous brain-derived neurotrophic factor from primary sensory neurons detected by ELISA in situ. $J$ Neurosci 20: $7417-7423$

Barg S, Olofsson CS, Schriever-Abeln J, Wendt A, Gebre-Medhin S, Renstrom E, Rorsman P (2002) Delay between fusion pore opening and peptide release from large dense-core vesicles in neuroendocrine cells. Neuron 33: 287-299

Barr F, Lambright DG (2010) Rab GEFs and GAPs. Curr Opin Cell Biol 22: 461-470

Barr FA, Short B (2003) Golgins in the structure and dynamics of the Golgi apparatus. Curr Opin Cell Biol 15: 405-413

Barrowman J, Bhandari D, Reinisch K, Ferro-Novick S (2010) TRAPP complexes in membrane traffic: convergence through a common Rab. Nat Rev Mol Cell Biol 11: 759763

Behrends C, Sowa ME, Gygi SP, Harper JW (2010) Network organization of the human autophagy system. Nature 466: 68-76

Boillee S, Peschanski M, Junier MP (2003) The wobbler mouse: a neurodegeneration jigsaw puzzle. Mol Neurobiol 28: 65-106

Bonifacino JS, Hierro A (2011) Transport according to GARP: receiving retrograde cargo at the trans-Golgi network. Trends Cell Biol 21: 159-167

Borgonovo B, Ouwendijk J, Solimena M (2006) Biogenesis of secretory granules. Curr Opin Cell Biol 18: 365-370

Brenner S (1974) The genetics of Caenorhabditis elegans. Genetics 77: 71-94

Bruinsma P, Spelbrink RG, Nothwehr SF (2004) Retrograde transport of the mannosyltransferase Och1p to the early Golgi requires a component of the COG transport complex. J Biol Chem 279: 39814-39823

Bruns D, Jahn R (1995) Real-time measurement of transmitter release from single synaptic vesicles. Nature 377: 62-65

Buffa L, Fuchs E, Pietropaolo M, Barr F, Solimena M (2008) ICA69 is a novel Rab2 effector regulating ER-Golgi trafficking in insulinoma cells. Eur J Cell Biol 87: 197-209

Buma P (1988) Synaptic and nonsynaptic release of neuromediators in the central nervous system. Acta Morphol Neerl Scand 26: 81-113 
Cai H, Reinisch K, Ferro-Novick S (2007) Coats, tethers, Rabs, and SNAREs work together to mediate the intracellular destination of a transport vesicle. Dev Cell 12: 671682

Callebaut I, de Gunzburg J, Goud B, Mornon JP (2001) RUN domains: a new family of domains involved in Ras-like GTPase signaling. Trends Biochem Sci 26: 79-83

Carroll KS, Hanna J, Simon I, Krise J, Barbero P, Pfeffer SR (2001) Role of Rab9 GTPase in facilitating receptor recruitment by TIP47. Science 292: 1373-1376

Chen L, Fu Y, Ren M, Xiao B, Rubin CS A RasGRP, C. elegans RGEF-1b, couples external stimuli to behavior by activating LET-60 (Ras) in sensory neurons. Neuron 70: 51-65

Chen L, Fu Y, Ren M, Xiao B, Rubin CS (2011) A RasGRP, C. elegans RGEF-1b, couples external stimuli to behavior by activating LET-60 (Ras) in sensory neurons. Neuron 70: 51-65

Chotard L, Mishra AK, Sylvain MA, Tuck S, Lambright DG, Rocheleau CE (2010) TBC2 regulates RAB-5/RAB-7-mediated endosomal trafficking in Caenorhabditis elegans. Mol Biol Cell 21: 2285-2296

Chun DK, McEwen JM, Burbea M, Kaplan JM (2008) UNC-108/Rab2 regulates postendocytic trafficking in Caenorhabditis elegans. Mol Biol Cell 19: 2682-2695

Chung CT, Niemela SL, Miller RH (1989) One-step preparation of competent Escherichia coli: transformation and storage of bacterial cells in the same solution. Proc Natl Acad Sci U S A 86: 2172-2175

Clabecq A, Henry JP, Darchen F (2000) Biochemical characterization of Rab3-GTPaseactivating protein reveals a mechanism similar to that of Ras-GAP. J Biol Chem 275: 31786-31791

Colomer V, Kicska GA, Rindler MJ (1996) Secretory granule content proteins and the luminal domains of granule membrane proteins aggregate in vitro at mildly acidic $\mathrm{pH}$. $J$ Biol Chem 271: 48-55

Conboy MJ, Cyert MS (2000) Luv1p/Rki1p/Tcs3p/Vps54p, a yeast protein that localizes to the late Golgi and early endosome, is required for normal vacuolar morphology. Mol Biol Cell 11: 2429-2443

Conibear E, Cleck JN, Stevens TH (2003) Vps51p mediates the association of the GARP (Vps52/53/54) complex with the late Golgi t-SNARE Tlg1p. Mol Biol Cell 14: 16101623 
Conibear E, Stevens TH (2000) Vps52p, Vps53p, and Vps54p form a novel multisubunit complex required for protein sorting at the yeast late Golgi. Mol Biol Cell 11: 305-323

Cool DR, Fenger M, Snell CR, Loh YP (1995) Identification of the sorting signal motif within pro-opiomelanocortin for the regulated secretory pathway. J Biol Chem 270: 8723-8729

Corda D, Hidalgo Carcedo C, Bonazzi M, Luini A, Spano S (2002) Molecular aspects of membrane fission in the secretory pathway. Cell Mol Life Sci 59: 1819-1832

Cormont M, Mari M, Galmiche A, Hofman P, Le Marchand-Brustel Y (2001) A FYVEfinger-containing protein, Rabip4, is a Rab4 effector involved in early endosomal traffic. Proc Natl Acad Sci U S A 98: 1637-1642

De Antoni A, Schmitzova J, Trepte HH, Gallwitz D, Albert S (2002) Significance of GTP hydrolysis in Ypt1p-regulated endoplasmic reticulum to Golgi transport revealed by the analysis of two novel Ypt1-GAPs. J Biol Chem 277: 41023-41031

De Camilli P, Jahn R (1990) Pathways to regulated exocytosis in neurons. Annu Rev Physiol 52: 625-645

Dhanvantari S, Loh YP (2000) Lipid raft association of carboxypeptidase E is necessary for its function as a regulated secretory pathway sorting receptor. $J$ Biol Chem 275: 29887-29893

Diaz E, Pfeffer SR (1998) TIP47: a cargo selection device for mannose 6-phosphate receptor trafficking. Cell 93: 433-443

Dittie AS, Hajibagheri N, Tooze SA (1996) The AP-1 adaptor complex binds to immature secretory granules from PC12 cells, and is regulated by ADP-ribosylation factor. J Cell Biol 132: 523-536

Dittie AS, Thomas L, Thomas G, Tooze SA (1997) Interaction of furin in immature secretory granules from neuroendocrine cells with the AP-1 adaptor complex is modulated by casein kinase II phosphorylation. EMBO J 16: 4859-4870

Du LL, Novick P (2001) Yeast rab GTPase-activating protein Gyp1p localizes to the Golgi apparatus and is a negative regulator of Ypt1p. Mol Biol Cell 12: 1215-1226

Dumas JJ, Zhu Z, Connolly JL, Lambright DG (1999) Structural basis of activation and GTP hydrolysis in Rab proteins. Structure 7: 413-423

Eaton BA, Haugwitz M, Lau D, Moore HP (2000) Biogenesis of regulated exocytotic carriers in neuroendocrine cells. J Neurosci 20: 7334-7344 
Echard A, Jollivet F, Martinez O, Lacapere JJ, Rousselet A, Janoueix-Lerosey I, Goud B (1998) Interaction of a Golgi-associated kinesin-like protein with Rab6. Science 279: 580-585

Edgar RC (2004) MUSCLE: multiple sequence alignment with high accuracy and high throughput. Nucleic Acids Res 32: 1792-1797

Edwards SL, Charlie NK, Richmond JE, Hegermann J, Eimer S, Miller KG (2009) Impaired dense core vesicle maturation in Caenorhabditis elegans mutants lacking Rab2. J Cell Biol 186: 881-895

Eiden LE, Giraud P, Dave JR, Hotchkiss AJ, Affolter HU (1984) Nicotinic receptor stimulation activates enkephalin release and biosynthesis in adrenal chromaffin cells. Nature 312: 661-663

Epp N, Rethmeier R, Kramer L, Ungermann C (2011) Membrane dynamics and fusion at late endosomes and vacuoles--Rab regulation, multisubunit tethering complexes and SNAREs. Eur J Cell Biol 90: 779-785

Etienne-Manneville S, Hall A (2002) Rho GTPases in cell biology. Nature 420: 629-635

Faitar SL, Dabbeekeh JT, Ranalli TA, Cowell JK (2005) EVI5 is a novel centrosomal protein that binds to alpha- and gamma-tubulin. Genomics 86: 594-605

Fares H, Greenwald I (2001a) Genetic analysis of endocytosis in Caenorhabditis elegans: coelomocyte uptake defective mutants. Genetics 159: 133-145

Fares H, Greenwald I (2001b) Regulation of endocytosis by CUP-5, the Caenorhabditis elegans mucolipin-1 homolog. Nat Genet 28: 64-68

Fernandes H, Franklin E, Recacha R, Houdusse A, Goud B, Khan AR (2009) Structural aspects of Rab6-effector complexes. Biochem Soc Trans 37: 1037-1041

Fire A, Xu S, Montgomery MK, Kostas SA, Driver SE, Mello CC (1998) Potent and specific genetic interference by double-stranded RNA in Caenorhabditis elegans. Nature 391: 806-811

Frasa MA, Koessmeier KT, Ahmadian MR, Braga VM (2012) Illuminating the functional and structural repertoire of human TBC/RABGAPs. Nat Rev Mol Cell Biol 13: 67-73

Fricker LD (1988) Activation and membrane binding of carboxypeptidase E. J Cell Biochem 38: 279-289

Fried G, Terenius L, Hokfelt T, Goldstein M (1985) Evidence for differential localization of noradrenaline and neuropeptide $\mathrm{Y}$ in neuronal storage vesicles isolated from rat vas deferens. J Neurosci 5: 450-458 
Frische EW, Pellis-van Berkel W, van Haaften G, Cuppen E, Plasterk RH, Tijsterman M, Bos JL, Zwartkruis FJ (2007) RAP-1 and the RAL-1/exocyst pathway coordinate hypodermal cell organization in Caenorhabditis elegans. EMBO J 26: 5083-5092

Frittoli E, Palamidessi A, Pizzigoni A, Lanzetti L, Garre M, Troglio F, Troilo A, Fukuda M, Di Fiore PP, Scita G, Confalonieri S (2008) The primate-specific protein TBC1D3 is required for optimal macropinocytosis in a novel ARF6-dependent pathway. Mol Biol Cell 19: 1304-1316

Frost A, Unger VM, De Camilli P (2009) The BAR domain superfamily: membranemolding macromolecules. Cell 137: 191-196

Fukuda M (2011) TBC proteins: GAPs for mammalian small GTPase Rab? Biosci Rep 31: $159-168$

Fukuda M, Kanno E, Ishibashi K, Itoh T (2008) Large scale screening for novel rab effectors reveals unexpected broad Rab binding specificity. Mol Cell Proteomics 7: 10311042

Fukuda M, Kobayashi H, Ishibashi K, Ohbayashi N (2011) Genome-wide investigation of the Rab binding activity of RUN domains: development of a novel tool that specifically traps GTP-Rab35. Cell Struct Funct 36: 155-170

Fukui K, Sasaki T, Imazumi K, Matsuura Y, Nakanishi H, Takai Y (1997) Isolation and characterization of a GTPase activating protein specific for the Rab3 subfamily of small G proteins. J Biol Chem 272: 4655-4658

Gallop JL, McMahon HT (2005) BAR domains and membrane curvature: bringing your curves to the BAR. Biochem Soc Symp: 223-231

Geppert M, Bolshakov VY, Siegelbaum SA, Takei K, De Camilli P, Hammer RE, Sudhof TC (1994) The role of Rab3A in neurotransmitter release. Nature 369: 493-497

Gillingham AK, Munro S (2003) Long coiled-coil proteins and membrane traffic. Biochim Biophys Acta 1641: 71-85

Gillingham AK, Munro S (2007) The small G proteins of the Arf family and their regulators. Annu Rev Cell Dev Biol 23: 579-611

Glombik MM, Gerdes HH (2000) Signal-mediated sorting of neuropeptides and prohormones: secretory granule biogenesis revisited. Biochimie 82: 315-326

Glowinski J, Kemel ML, Desban M, Gauchy C, Lavielle S, Chassaing G, Beaujouan JC, Tremblay L (1993) Distinct presynaptic control of dopamine release in striosomal- and matrix-enriched areas of the rat striatum by selective agonists of NK1, NK2 and NK3 tachykinin receptors. Regul Pept 46: 124-128 
Godi A, Di Campli A, Konstantakopoulos A, Di Tullio G, Alessi DR, Kular GS, Daniele T, Marra P, Lucocq JM, De Matteis MA (2004) FAPPs control Golgi-to-cell-surface membrane traffic by binding to ARF and PtdIns(4)P. Nat Cell Biol 6: 393-404

Gracheva EO, Burdina AO, Touroutine D, Berthelot-Grosjean M, Parekh H, Richmond JE (2007) Tomosyn negatively regulates CAPS-dependent peptide release at Caenorhabditis elegans synapses. J Neurosci 27: 10176-10184

Gracheva EO, Hadwiger G, Nonet ML, Richmond JE (2008) Direct interactions between C. elegans RAB-3 and Rim provide a mechanism to target vesicles to the presynaptic density. Neurosci Lett 444: 137-142

Haas AK, Fuchs E, Kopajtich R, Barr FA (2005) A GTPase-activating protein controls Rab5 function in endocytic trafficking. Nat Cell Biol 7: 887-893

Haas AK, Yoshimura S, Stephens DJ, Preisinger C, Fuchs E, Barr FA (2007) Analysis of GTPase-activating proteins: Rab1 and Rab43 are key Rabs required to maintain a functional Golgi complex in human cells. J Cell Sci 120: 2997-3010

Habermann B (2004) The BAR-domain family of proteins: a case of bending and binding? EMBO Rep 5: 250-255

Hagan S, Niswender KD (2012) Neuroendocrine regulation of food intake. Pediatr Blood Cancer 58: 149-153

Halban PA, Irminger JC (1994) Sorting and processing of secretory proteins. Biochem J 299 ( Pt 1): 1-18

Hales CM, Vaerman JP, Goldenring JR (2002) Rab11 family interacting protein 2 associates with Myosin $\mathrm{Vb}$ and regulates plasma membrane recycling. $J$ Biol Chem 277: 50415-50421

Hannemann M, Sasidharan N, Hegermann J, Kutscher LM, Koenig S, Eimer S (2012) TBC-8, a Putative RAB-2 GAP, Regulates Dense Core Vesicle Maturation in Caenorhabditis elegans. PLoS Genet 8: e1002722

Harashima S, Clark A, Christie MR, Notkins AL (2005) The dense core transmembrane vesicle protein IA-2 is a regulator of vesicle number and insulin secretion. Proc Natl Acad Sci U S A 102: 8704-8709

Harata N, Pyle JL, Aravanis AM, Mozhayeva M, Kavalali ET, Tsien RW (2001) Limited numbers of recycling vesicles in small CNS nerve terminals: implications for neural signaling and vesicular cycling. Trends Neurosci 24: 637-643

He B, Guo W (2009) The exocyst complex in polarized exocytosis. Curr Opin Cell Biol 21: $537-542$ 
Ho SY, Lorent K, Pack M, Farber SA (2006) Zebrafish fat-free is required for intestinal lipid absorption and Golgi apparatus structure. Cell Metab 3: 289-300

Hobert O, Mori I, Yamashita Y, Honda H, Ohshima Y, Liu Y, Ruvkun G (1997) Regulation of interneuron function in the C. elegans thermoregulatory pathway by the ttx-3 LIM homeobox gene. Neuron 19: 345-357

Hokanson DE, Bretscher AP (2012) EPI64 interacts with Slp1/JFC1 to coordinate Rab8a and Arf6 membrane trafficking. Mol Biol Cell 23: 701-715

Hokfelt T (1991) Neuropeptides in perspective: the last ten years. Neuron 7: 867-879

Hopkins SJ, Rothwell NJ (1995) Cytokines and the nervous system. I: Expression and recognition. Trends Neurosci 18: $83-88$

Hosaka M, Watanabe T (2010) Secretogranin III: a bridge between core hormone aggregates and the secretory granule membrane. Endocr J 57: 275-286

Hosaka M, Watanabe T, Sakai Y, Kato T, Takeuchi T (2005) Interaction between secretogranin III and carboxypeptidase $\mathrm{E}$ facilitates prohormone sorting within secretory granules. J Cell Sci 118: 4785-4795

Hume AN, Collinson LM, Rapak A, Gomes AQ, Hopkins CR, Seabra MC (2001) Rab27a regulates the peripheral distribution of melanosomes in melanocytes. J Cell Biol 152: $795-808$

Husson SJ, Clynen E, Baggerman G, Janssen T, Schoofs L (2006) Defective processing of neuropeptide precursors in Caenorhabditis elegans lacking proprotein convertase 2 (KPC-2/EGL-3): mutant analysis by mass spectrometry. J Neurochem 98: 1999-2012

Hutagalung AH, Novick PJ (2011) Role of rab GTPases in membrane traffic and cell physiology. Physiol Rev 91: 119-149

Huttner WB, Gerdes HH, Rosa P (1991) The granin (chromogranin/secretogranin) family. Trends Biochem Sci 16: 27-30

Ishibashi K, Kanno E, Itoh T, Fukuda M (2009) Identification and characterization of a novel Tre-2/Bub2/Cdc16 (TBC) protein that possesses Rab3A-GAP activity. Genes Cells 14: $41-52$

Itoh T, Kanno E, Uemura T, Waguri S, Fukuda M (2011) OATL1, a novel autophagosome-resident Rab33B-GAP, regulates autophagosomal maturation. J Cell Biol 192: $839-853$

Itzen A, Goody RS (2011) GTPases involved in vesicular trafficking: structures and mechanisms. Semin Cell Dev Biol 22: 48-56 
Jacob TC, Kaplan JM (2003) The EGL-21 carboxypeptidase E facilitates acetylcholine release at Caenorhabditis elegans neuromuscular junctions. J Neurosci 23: 2122-2130 Jahn R, Scheller RH (2006) SNAREs--engines for membrane fusion. Nat Rev Mol Cell Biol 7: 631-643

Janoueix-Lerosey I, Jollivet F, Camonis J, Marche PN, Goud B (1995) Two-hybrid system screen with the small GTP-binding protein Rab6. Identification of a novel mouse GDP dissociation inhibitor isoform and two other potential partners of Rab6. J Biol Chem 270: $14801-14808$

Janoueix-Lerosey I, Pasheva E, de Tand MF, Tavitian A, de Gunzburg J (1998) Identification of a specific effector of the small GTP-binding protein Rap2. Eur $J$ Biochem 252: 290-298

Jones S, Newman C, Liu F, Segev N (2000) The TRAPP complex is a nucleotide exchanger for Ypt1 and Ypt31/32. Mol Biol Cell 11: 4403-4411

Kakhlon O, Sakya P, Larijani B, Watson R, Tooze SA (2006) GGA function is required for maturation of neuroendocrine secretory granules. EMBO J 25: 1590-1602

Kamath RS, Fraser AG, Dong Y, Poulin G, Durbin R, Gotta M, Kanapin A, Le Bot N, Moreno S, Sohrmann M, Welchman DP, Zipperlen P, Ahringer J (2003) Systematic functional analysis of the Caenorhabditis elegans genome using RNAi. Nature 421: 231237

Kamath RS, Martinez-Campos M, Zipperlen P, Fraser AG, Ahringer J (2001) Effectiveness of specific RNA-mediated interference through ingested double-stranded RNA in Caenorhabditis elegans. Genome Biol 2: RESEARCH0002

Kamena F, Spang A (2004) Tip20p prohibits back-fusion of COPII vesicles with the endoplasmic reticulum. Science 304: 286-289

Kanno E, Ishibashi K, Kobayashi H, Matsui T, Ohbayashi N, Fukuda M (2010) Comprehensive screening for novel rab-binding proteins by GST pull-down assay using 60 different mammalian Rabs. Traffic 11: 491-507

Karhunen T, Vilim FS, Alexeeva V, Weiss KR, Church PJ (2001) Targeting of peptidergic vesicles in cotransmitting terminals. J Neurosci 21: RC127

Kass J, Jacob TC, Kim P, Kaplan JM (2001) The EGL-3 proprotein convertase regulates mechanosensory responses of Caenorhabditis elegans. J Neurosci 21: 9265-9272

Kim T, Gondre-Lewis MC, Arnaoutova I, Loh YP (2006) Dense-core secretory granule biogenesis. Physiology (Bethesda) 21: 124-133 
Kim T, Loh YP (2006) Protease nexin-1 promotes secretory granule biogenesis by preventing granule protein degradation. Mol Biol Cell 17: 789-798

Kim T, Zhang CF, Sun Z, Wu H, Loh YP (2005) Chromogranin A deficiency in transgenic mice leads to aberrant chromaffin granule biogenesis. J Neurosci 25: 69586961

Knoch KP, Bergert H, Borgonovo B, Saeger HD, Altkruger A, Verkade P, Solimena M (2004) Polypyrimidine tract-binding protein promotes insulin secretory granule biogenesis. Nat Cell Biol 6: 207-214

Kramer JM, French RP, Park EC, Johnson JJ (1990) The Caenorhabditis elegans rol-6 gene, which interacts with the sqt- 1 collagen gene to determine organismal morphology, encodes a collagen. Mol Cell Biol 10: 2081-2089

Kraynack BA, Chan A, Rosenthal E, Essid M, Umansky B, Waters MG, Schmitt HD (2005) Dsl1p, Tip20p, and the novel Dsl3(Sec39) protein are required for the stability of the Q/t-SNARE complex at the endoplasmic reticulum in yeast. Mol Biol Cell 16: 39633977

Kukimoto-Niino M, Takagi T, Akasaka R, Murayama K, Uchikubo-Kamo T, Terada T, Inoue M, Watanabe S, Tanaka A, Hayashizaki Y, Kigawa T, Shirouzu M, Yokoyama S (2006) Crystal structure of the RUN domain of the RAP2-interacting protein x. J Biol Chem 281: 31843-31853

Kumar U (2011) Cross-talk and modulation of signaling between somatostatin and growth factor receptors. Endocrine 40: 168-180

Kupfermann I (1991) Functional studies of cotransmission. Physiol Rev 71: 683-732

Lai T, Garriga G (2004) The conserved kinase UNC-51 acts with VAB-8 and UNC-14 to regulate axon outgrowth in C. elegans. Development 131: 5991-6000

Lee IK, Kim KS, Kim H, Lee JY, Ryu CH, Chun HJ, Lee KU, Lim Y, Kim YH, Huh PW, Lee KH, Han SI, Jun TY, Rha HK (2004) MAP, a protein interacting with a tumor suppressor, merlin, through the run domain. Biochem Biophys Res Commun 325: 774-783

Lee MT, Mishra A, Lambright DG (2009) Structural mechanisms for regulation of membrane traffic by rab GTPases. Traffic 10: 1377-1389

Li C (2005) The ever-expanding neuropeptide gene families in the nematode Caenorhabditis elegans. Parasitology 131 Suppl: S109-127

Li C, Takei K, Geppert M, Daniell L, Stenius K, Chapman ER, Jahn R, De Camilli P, Sudhof TC (1994) Synaptic targeting of rabphilin-3A, a synaptic vesicle Ca2+/phospholipid-binding protein, depends on rab3A/3C. Neuron 13: 885-898 
Li HY, Cao K, Zheng Y (2003) Ran in the spindle checkpoint: a new function for a versatile GTPase. Trends Cell Biol 13: 553-557

Lian JP, Stone S, Jiang Y, Lyons P, Ferro-Novick S (1994) Ypt1p implicated in vSNARE activation. Nature 372: 698-701

Liewen H, Meinhold-Heerlein I, Oliveira V, Schwarzenbacher R, Luo G, Wadle A, Jung M, Pfreundschuh M, Stenner-Liewen F (2005) Characterization of the human GARP (Golgi associated retrograde protein) complex. Exp Cell Res 306: 24-34

Loh YP, Maldonado A, Zhang C, Tam WH, Cawley N (2002) Mechanism of sorting proopiomelanocortin and proenkephalin to the regulated secretory pathway of neuroendocrine cells. Ann N Y Acad Sci 971: 416-425

Lombardi D, Soldati T, Riederer MA, Goda Y, Zerial M, Pfeffer SR (1993) Rab9 functions in transport between late endosomes and the trans Golgi network. EMBO J 12: $677-682$

Lu J, Li Q, Xie H, Chen ZJ, Borovitskaya AE, Maclaren NK, Notkins AL, Lan MS (1996) Identification of a second transmembrane protein tyrosine phosphatase, IA-2beta, as an autoantigen in insulin-dependent diabetes mellitus: precursor of the 37-kDa tryptic fragment. Proc Natl Acad Sci U S A 93: 2307-2311

Lu Q, Zhang Y, Hu T, Guo P, Li W, Wang X (2008) C. elegans Rab GTPase 2 is required for the degradation of apoptotic cells. Development 135: 1069-1080

Lundberg JM (1996) Pharmacology of cotransmission in the autonomic nervous system: integrative aspects on amines, neuropeptides, adenosine triphosphate, amino acids and nitric oxide. Pharmacol Rev 48: 113-178

Lundquist EA (2006) Small GTPases. WormBook: 1-18

Luo L, Hannemann M, Koenig S, Hegermann J, Ailion M, Cho MK, Sasidharan N, Zweckstetter M, Rensing SA, Eimer S (2011) The Caenorhabditis elegans GARP complex contains the conserved Vps51 subunit and is required to maintain lysosomal morphology. Mol Biol Cell 22: 2564-2578

Lupas AN, Gruber M (2005) The structure of alpha-helical coiled coils. Adv Protein Chem 70: 37-78

Lynch-Day MA, Bhandari D, Menon S, Huang J, Cai H, Bartholomew CR, Brumell JH, Ferro-Novick S, Klionsky DJ (2010) Trs85 directs a Ypt1 GEF, TRAPPIII, to the phagophore to promote autophagy. Proc Natl Acad Sci U S A 107: 7811-7816

MacDonald JI, Kubu CJ, Meakin SO (2004) Nesca, a novel adapter, translocates to the nuclear envelope and regulates neurotrophin-induced neurite outgrowth. J Cell Biol 164: 851-862 
Mahapatra NR, Mahata M, O'Connor DT, Mahata SK (2003) Secretin activation of chromogranin A gene transcription. Identification of the signaling pathways in cis and in trans. J Biol Chem 278: 19986-19994

Mahapatra NR, O'Connor DT, Vaingankar SM, Hikim AP, Mahata M, Ray S, Staite E, Wu H, Gu Y, Dalton N, Kennedy BP, Ziegler MG, Ross J, Mahata SK (2005) Hypertension from targeted ablation of chromogranin A can be rescued by the human ortholog. J Clin Invest 115: 1942-1952

Mahata SK, Mahapatra NR, Mahata M, Wang TC, Kennedy BP, Ziegler MG, O'Connor DT (2003) Catecholamine secretory vesicle stimulus-transcription coupling in vivo. Demonstration by a novel transgenic promoter/photoprotein reporter and inhibition of secretion and transcription by the chromogranin A fragment catestatin. J Biol Chem 278: 32058-32067

Mahoney TR, Liu Q, Itoh T, Luo S, Hadwiger G, Vincent R, Wang ZW, Fukuda M, Nonet ML (2006) Regulation of synaptic transmission by RAB-3 and RAB-27 in Caenorhabditis elegans. Mol Biol Cell 17: 2617-2625

Malcangio M, Bowery NG (1999) Peptide autoreceptors: does an autoreceptor for substance P exist? Trends Pharmacol Sci 20: 405-407

Mangahas PM, Yu X, Miller KG, Zhou Z (2008) The small GTPase Rab2 functions in the removal of apoptotic cells in Caenorhabditis elegans. J Cell Biol 180: 357-373

Marat AL, Dokainish H, McPherson PS (2011) DENN domain proteins: regulators of Rab GTPases. J Biol Chem 286: 13791-13800

Mari M, Macia E, Le Marchand-Brustel Y, Cormont M (2001) Role of the FYVE finger and the RUN domain for the subcellular localization of Rabip4. J Biol Chem 276: 4250142508

Mello C, Fire A (1995) DNA transformation. Methods Cell Biol 48: 451-482

Mens WB, Witter A, van Wimersma Greidanus TB (1983) Penetration of neurohypophyseal hormones from plasma into cerebrospinal fluid (CSF): half-times of disappearance of these neuropeptides from CSF. Brain Res 262: 143-149

Merighi A (2002) Costorage and coexistence of neuropeptides in the mammalian CNS. Prog Neurobiol 66: 161-190

Messinis IE, Messini CI, Dafopoulos K (2010) The role of gonadotropins in the follicular phase. Ann N Y Acad Sci 1205: 5-11 
Milburn MV, Tong L, deVos AM, Brunger A, Yamaizumi Z, Nishimura S, Kim SH (1990) Molecular switch for signal transduction: structural differences between active and inactive forms of protooncogenic ras proteins. Science 247: 939-945

Milgram SL, Kho ST, Martin GV, Mains RE, Eipper BA (1997) Localization of integral membrane peptidylglycine alpha-amidating monooxygenase in neuroendocrine cells. $J$ Cell Sci 110 ( Pt 6): 695-706

Molloy SS, Thomas L, VanSlyke JK, Stenberg PE, Thomas G (1994) Intracellular trafficking and activation of the furin proprotein convertase: localization to the TGN and recycling from the cell surface. $E M B O J$ 13: 18-33

Morvan J, Tooze SA (2008) Discovery and progress in our understanding of the regulated secretory pathway in neuroendocrine cells. Histochem Cell Biol 129: 243-252

Moyer BD, Allan BB, Balch WE (2001) Rab1 interaction with a GM130 effector complex regulates COPII vesicle cis--Golgi tethering. Traffic 2: 268-276

Mukhopadhyay A, Deplancke B, Walhout AJ, Tissenbaum HA (2005) C. elegans tubby regulates life span and fat storage by two independent mechanisms. Cell Metab 2: 35-42

Munro S (2011) The golgin coiled-coil proteins of the Golgi apparatus. Cold Spring Harb Perspect Biol 3

Nagano F, Sasaki T, Fukui K, Asakura T, Imazumi K, Takai Y (1998) Molecular cloning and characterization of the noncatalytic subunit of the Rab3 subfamily-specific GTPaseactivating protein. $J$ Biol Chem 273: 24781-24785

Neuwald AF (1997) A shared domain between a spindle assembly checkpoint protein and Ypt/Rab-specific GTPase-activators. Trends Biochem Sci 22: 243-244

Ng EL, Tang BL (2008) Rab GTPases and their roles in brain neurons and glia. Brain Res Rev 58: 236-246

Nottingham RM, Ganley IG, Barr FA, Lambright DG, Pfeffer SR (2011) RUTBC1 protein, a Rab9A effector that activates GTP hydrolysis by Rab32 and Rab33B proteins. J Biol Chem 286: 33213-33222

Ogura K, Shirakawa M, Barnes TM, Hekimi S, Ohshima Y (1997) The UNC-14 protein required for axonal elongation and guidance in Caenorhabditis elegans interacts with the serine/threonine kinase UNC-51. Genes Dev 11: 1801-1811

Oka T, Krieger M (2005) Multi-component protein complexes and Golgi membrane trafficking. J Biochem 137: 109-114 
Pan X, Eathiraj S, Munson M, Lambright DG (2006) TBC-domain GAPs for Rab GTPases accelerate GTP hydrolysis by a dual-finger mechanism. Nature 442: 303-306

Panic B, Whyte JR, Munro S (2003) The ARF-like GTPases Arl1p and Arl3p act in a pathway that interacts with vesicle-tethering factors at the Golgi apparatus. Curr Biol 13: 405-410

Park JJ, Koshimizu H, Loh YP (2009) Biogenesis and transport of secretory granules to release site in neuroendocrine cells. J Mol Neurosci 37: 151-159

Pavlos NJ, Gronborg M, Riedel D, Chua JJ, Boyken J, Kloepper TH, Urlaub H, Rizzoli SO, Jahn R (2010) Quantitative analysis of synaptic vesicle Rabs uncovers distinct yet overlapping roles for Rab3a and Rab27b in Ca2+-triggered exocytosis. J Neurosci 30: 13441-13453

Pelletier G, Steinbusch HW, Verhofstad AA (1981) Immunoreactive substance P and serotonin present in the same dense-core vesicles. Nature 293: 71-72

Peplowska K, Markgraf DF, Ostrowicz CW, Bange G, Ungermann C (2007) The CORVET tethering complex interacts with the yeast Rab5 homolog Vps21 and is involved in endo-lysosomal biogenesis. Dev Cell 12: 739-750

Pereira-Leal JB, Seabra MC (2000) The mammalian Rab family of small GTPases: definition of family and subfamily sequence motifs suggests a mechanism for functional specificity in the Ras superfamily. J Mol Biol 301: 1077-1087

Perez-Victoria FJ, Abascal-Palacios G, Tascon I, Kajava A, Magadan JG, Pioro EP, Bonifacino JS, Hierro A (2010a) Structural basis for the wobbler mouse neurodegenerative disorder caused by mutation in the Vps54 subunit of the GARP complex. Proc Natl Acad Sci U S A 107: 12860-12865

Perez-Victoria FJ, Bonifacino JS (2009) Dual roles of the mammalian GARP complex in tethering and SNARE complex assembly at the trans-golgi network. Mol Cell Biol 29: 5251-5263

Perez-Victoria FJ, Mardones GA, Bonifacino JS (2008) Requirement of the human GARP complex for mannose 6-phosphate-receptor-dependent sorting of cathepsin D to lysosomes. Mol Biol Cell 19: 2350-2362

Perez-Victoria FJ, Schindler C, Magadan JG, Mardones GA, Delevoye C, Romao M, Raposo G, Bonifacino JS (2010b) Ang2/fat-free is a conserved subunit of the Golgiassociated retrograde protein complex. Mol Biol Cell 21: 3386-3395

Pfeffer S (2005) Filling the Rab GAP. Nat Cell Biol 7: 856-857 
Pierce SB, Costa M, Wisotzkey R, Devadhar S, Homburger SA, Buchman AR, Ferguson KC, Heller J, Platt DM, Pasquinelli AA, Liu LX, Doberstein SK, Ruvkun G (2001) Regulation of DAF-2 receptor signaling by human insulin and ins-1, a member of the unusually large and diverse C. elegans insulin gene family. Genes Dev 15: 672-686

Quenneville NR, Chao TY, McCaffery JM, Conibear E (2006) Domains within the GARP subunit Vps54 confer separate functions in complex assembly and early endosome recognition. Mol Biol Cell 17: 1859-1870

Ram RJ, Li B, Kaiser CA (2002) Identification of Sec36p, Sec37p, and Sec38p: components of yeast complex that contains Sec34p and Sec35p. Mol Biol Cell 13: 14841500

Rao Y, Haucke V (2011) Membrane shaping by the Bin/amphiphysin/Rvs (BAR) domain protein superfamily. Cell Mol Life Sci 68: 3983-3993

Recacha R, Boulet A, Jollivet F, Monier S, Houdusse A, Goud B, Khan AR (2009) Structural basis for recruitment of Rab6-interacting protein 1 to Golgi via a RUN domain. Structure 17: 21-30

Reynolds ES (1963) The use of lead citrate at high $\mathrm{pH}$ as an electron-opaque stain in electron microscopy. J Cell Biol 17: 208-212

Richmond J (2005) Synaptic function. WormBook: 1-14

Riedel D, Antonin W, Fernandez-Chacon R, Alvarez de Toledo G, Jo T, Geppert M, Valentijn JA, Valentijn K, Jamieson JD, Sudhof TC, Jahn R (2002) Rab3D is not required for exocrine exocytosis but for maintenance of normally sized secretory granules. Mol Cell Biol 22: 6487-6497

Rivera-Molina FE, Novick PJ (2009a) A Rab GAP cascade defines the boundary between two Rab GTPases on the secretory pathway. Proc Natl Acad Sci USA 106: 14408-14413

Rivera-Molina FE, Novick PJ (2009b) A Rab GAP cascade defines the boundary between two Rab GTPases on the secretory pathway. Proc Natl Acad Sci U S A 106: 14408-14413

Rostaing P, Weimer RM, Jorgensen EM, Triller A, Bessereau JL (2004) Preservation of immunoreactivity and fine structure of adult C. elegans tissues using high-pressure freezing. J Histochem Cytochem 52: 1-12

Rothwell NJ, Hopkins SJ (1995) Cytokines and the nervous system II: Actions and mechanisms of action. Trends Neurosci 18: 130-136 
Rybin V, Ullrich O, Rubino M, Alexandrov K, Simon I, Seabra MC, Goody R, Zerial M (1996) GTPase activity of Rab5 acts as a timer for endocytic membrane fusion. Nature 383: 266-269

Sacher M, Kim YG, Lavie A, Oh BH, Segev N (2008) The TRAPP complex: insights into its architecture and function. Traffic 9: 2032-2042

Sakamoto R, Byrd DT, Brown HM, Hisamoto N, Matsumoto K, Jin Y (2005) The Caenorhabditis elegans UNC-14 RUN domain protein binds to the kinesin-1 and UNC-16 complex and regulates synaptic vesicle localization. Mol Biol Cell 16: 483-496

Sakane A, Manabe S, Ishizaki H, Tanaka-Okamoto M, Kiyokage E, Toida K, Yoshida T, Miyoshi J, Kamiya H, Takai Y, Sasaki T (2006) Rab3 GTPase-activating protein regulates synaptic transmission and plasticity through the inactivation of Rab3. Proc Natl Acad Sci U S A 103: 10029-10034

Salio C, Lossi L, Ferrini F, Merighi A (2006) Neuropeptides as synaptic transmitters. Cell Tissue Res 326: 583-598

Sambrook, J., and Russel, D.W. (2001). Molecular Cloning: A laboratory manual. Cold Spring Harbor Laboratory Press 1.

Schardt A, Brinkmann BG, Mitkovski M, Sereda MW, Werner HB, Nave KA (2009) The SNARE protein SNAP-29 interacts with the GTPase Rab3A: Implications for membrane trafficking in myelinating glia. J Neurosci Res 87: 3465-3479

Schlichting I, Almo SC, Rapp G, Wilson K, Petratos K, Lentfer A, Wittinghofer A, Kabsch W, Pai EF, Petsko GA, et al. (1990) Time-resolved X-ray crystallographic study of the conformational change in Ha-Ras p21 protein on GTP hydrolysis. Nature 345: 309-315

Schmitt-John T, Drepper C, Mussmann A, Hahn P, Kuhlmann M, Thiel C, Hafner M, Lengeling A, Heimann P, Jones JM, Meisler MH, Jockusch H (2005) Mutation of Vps54 causes motor neuron disease and defective spermiogenesis in the wobbler mouse. Nat Genet 37: 1213-1215

Schoonderwoert VT, Martens GJ (2001) Proton pumping in the secretory pathway. $J$ Membr Biol 182: 159-169

Schumacher B, Schertel C, Wittenburg N, Tuck S, Mitani S, Gartner A, Conradt B, Shaham S (2005) C. elegans ced-13 can promote apoptosis and is induced in response to DNA damage. Cell Death Differ 12: 153-161

Sclafani A, Chen S, Rivera-Molina F, Reinisch K, Novick P, Ferro-Novick S (2010) Establishing a Role for the GTPase Ypt1p at the Late Golgi. Traffic 11: 520-532

Segev N (2001) Ypt/rab gtpases: regulators of protein trafficking. Sci STKE 2001: re11 
Seidah NG, Chretien M (1999) Proprotein and prohormone convertases: a family of subtilases generating diverse bioactive polypeptides. Brain Res 848: 45-62

Segev N (2011) GTPases in intracellular trafficking: an overview. Semin Cell Dev Biol 22: $1-2$

Sheffield P, Garrard S, Derewenda Z (1999) Overcoming expression and purification problems of RhoGDI using a family of "parallel" expression vectors. Protein Expr Purif 15: $34-39$

Shemesh T, Luini A, Malhotra V, Burger KN, Kozlov MM (2003) Prefission constriction of Golgi tubular carriers driven by local lipid metabolism: a theoretical model. Biophys $J$ 85: 3813-3827

Shi A, Chen CC, Banerjee R, Glodowski D, Audhya A, Rongo C, Grant BD (2010) EHBP-1 functions with RAB-10 during endocytic recycling in Caenorhabditis elegans. Mol Biol Cell 21: 2930-2943

Sieburth D, Ch'ng Q, Dybbs M, Tavazoie M, Kennedy S, Wang D, Dupuy D, Rual JF, Hill DE, Vidal M, Ruvkun G, Kaplan JM (2005) Systematic analysis of genes required for synapse structure and function. Nature 436: 510-517

Sieburth D, Madison JM, Kaplan JM (2007) PKC-1 regulates secretion of neuropeptides. Nat Neurosci 10: 49-57

Siniossoglou S, Pelham HR (2001) An effector of Ypt6p binds the SNARE Tlg1p and mediates selective fusion of vesicles with late Golgi membranes. EMBO J 20: 5991-5998

Siniossoglou S, Pelham HR (2002) Vps51p links the VFT complex to the SNARE Tlg1p. J Biol Chem 277: 48318-48324

Sinka R, Gillingham AK, Kondylis V, Munro S (2008) Golgi coiled-coil proteins contain multiple binding sites for Rab family G proteins. J Cell Biol 183: 607-615

Sivaram MV, Furgason ML, Brewer DN, Munson M (2006) The structure of the exocyst subunit Sec6p defines a conserved architecture with diverse roles. Nat Struct Mol Biol 13: $555-556$

Soldati T, Shapiro AD, Svejstrup AB, Pfeffer SR (1994) Membrane targeting of the small GTPase Rab9 is accompanied by nucleotide exchange. Nature 369: 76-78

Stenmark H (2009) Rab GTPases as coordinators of vesicle traffic. Nat Rev Mol Cell Biol 10: $513-525$ 
Strom M, Hume AN, Tarafder AK, Barkagianni E, Seabra MC (2002) A family of Rab27-binding proteins. Melanophilin links Rab27a and myosin Va function in melanosome transport. J Biol Chem 277: 25423-25430

Sudhof TC, Rothman JE (2009) Membrane fusion: grappling with SNARE and SM proteins. Science 323: 474-477

Sumakovic M, Hegermann J, Luo L, Husson SJ, Schwarze K, Olendrowitz C, Schoofs L, Richmond J, Eimer S (2009) UNC-108/RAB-2 and its effector RIC-19 are involved in dense core vesicle maturation in Caenorhabditis elegans. J Cell Biol 186: 897-914

Sztul E, Lupashin V (2006) Role of tethering factors in secretory membrane traffic. Am $J$ Physiol Cell Physiol 290: C11-26

Tanaka S, Yora T, Nakayama K, Inoue K, Kurosumi K (1997) Proteolytic processing of pro-opiomelanocortin occurs in acidifying secretory granules of AtT-20 cells. $J$ Histochem Cytochem 45: 425-436

Tang K, Wu H, Mahata SK, Mahata M, Gill BM, Parmer RJ, O'Connor DT (1997) Stimulus coupling to transcription versus secretion in pheochromocytoma cells. Convergent and divergent signal transduction pathways and the crucial roles for route of cytosolic calcium entry and protein kinase C. J Clin Invest 100: 1180-1192

Taupenot L, Harper KL, Mahapatra NR, Parmer RJ, Mahata SK, O'Connor DT (2002) Identification of a novel sorting determinant for the regulated pathway in the secretory protein chromogranin A. J Cell Sci 115: 4827-4841

Taupenot L, Harper KL, O'Connor DT (2005) Role of H+-ATPase-mediated acidification in sorting and release of the regulated secretory protein chromogranin A: evidence for a vesiculogenic function. J Biol Chem 280: 3885-3897

Theodosis DT, Montagnese C, Rodriguez F, Vincent JD, Poulain DA (1986) Oxytocin induces morphological plasticity in the adult hypothalamo-neurohypophysial system. Nature 322: 738-740

Timmons L, Court DL, Fire A (2001) Ingestion of bacterially expressed dsRNAs can produce specific and potent genetic interference in Caenorhabditis elegans. Gene 263: 103-112

Tooze SA (1991) Biogenesis of secretory granules. Implications arising from the immature secretory granule in the regulated pathway of secretion. FEBS Lett 285: 220224

Tooze SA (1998) Biogenesis of secretory granules in the trans-Golgi network of neuroendocrine and endocrine cells. Biochim Biophys Acta 1404: 231-244 
Tooze SA, Flatmark T, Tooze J, Huttner WB (1991) Characterization of the immature secretory granule, an intermediate in granule biogenesis. J Cell Biol 115: 1491-1503

Tooze SA, Martens GJ, Huttner WB (2001) Secretory granule biogenesis: rafting to the SNARE. Trends Cell Biol 11: 116-122

Trent C, Tsuing N, Horvitz HR (1983) Egg-laying defective mutants of the nematode Caenorhabditis elegans. Genetics 104: 619-647

Tripathi A, Ren Y, Jeffrey PD, Hughson FM (2009) Structural characterization of Tip20p and Dsl1p, subunits of the Dsl1p vesicle tethering complex. Nat Struct Mol Biol 16: 114123

Tsuboi T, Rutter GA (2003) Insulin secretion by 'kiss-and-run' exocytosis in clonal pancreatic islet beta-cells. Biochem Soc Trans 31: 833-836

Ullrich O, Horiuchi H, Bucci C, Zerial M (1994) Membrane association of Rab5 mediated by GDP-dissociation inhibitor and accompanied by GDP/GTP exchange. Nature 368: 157-160

Ullrich O, Stenmark H, Alexandrov K, Huber LA, Kaibuchi K, Sasaki T, Takai Y, Zerial M (1993) Rab GDP dissociation inhibitor as a general regulator for the membrane association of rab proteins. J Biol Chem 268: 18143-18150

Ungar D, Hughson FM (2003) SNARE protein structure and function. Annu Rev Cell Dev Biol 19: 493-517

Ungar D, Oka T, Brittle EE, Vasile E, Lupashin VV, Chatterton JE, Heuser JE, Krieger M, Waters MG (2002) Characterization of a mammalian Golgi-localized protein complex, COG, that is required for normal Golgi morphology and function. J Cell Biol 157: $405-415$

VanRheenen SM, Cao X, Lupashin VV, Barlowe C, Waters MG (1998) Sec35p, a novel peripheral membrane protein, is required for ER to Golgi vesicle docking. J Cell Biol 141: $1107-1119$

VanRheenen SM, Cao X, Sapperstein SK, Chiang EC, Lupashin VV, Barlowe C, Waters MG (1999) Sec34p, a protein required for vesicle tethering to the yeast Golgi apparatus, is in a complex with Sec35p. J Cell Biol 147: 729-742

Vasan N, Hutagalung A, Novick P, Reinisch KM (2010) Structure of a C-terminal fragment of its Vps53 subunit suggests similarity of Golgi-associated retrograde protein (GARP) complex to a family of tethering complexes. Proc Natl Acad Sci U S A 107: 14176-14181 
Verhage M, McMahon HT, Ghijsen WE, Boomsma F, Scholten G, Wiegant VM, Nicholls DG (1991) Differential release of amino acids, neuropeptides, and catecholamines from isolated nerve terminals. Neuron 6: 517-524

Wasmeier C, Hutton JC (1996) Molecular cloning of phogrin, a protein-tyrosine phosphatase homologue localized to insulin secretory granule membranes. J Biol Chem 271: $18161-18170$

Weis K (2003) Regulating access to the genome: nucleocytoplasmic transport throughout the cell cycle. Cell 112: 441-451

Wennerberg K, Rossman KL, Der CJ (2005) The Ras superfamily at a glance. J Cell Sci 118: $843-846$

Whyte JR, Munro S (2001) The Sec34/35 Golgi transport complex is related to the exocyst, defining a family of complexes involved in multiple steps of membrane traffic. Dev Cell 1: 527-537

Wilson R, Ainscough R, Anderson K, Baynes C, Berks M, Bonfield J, Burton J, Connell $\mathrm{M}$, Copsey T, Cooper J, et al. (1994) $2.2 \mathrm{Mb}$ of contiguous nucleotide sequence from chromosome III of C. elegans. Nature 368: 32-38

Wu MM, Grabe M, Adams S, Tsien RY, Moore HP, Machen TE (2001a) Mechanisms of pH regulation in the regulated secretory pathway. J Biol Chem 276: 33027-33035

Wu X, Rao K, Bowers MB, Copeland NG, Jenkins NA, Hammer JA, 3rd (2001b) Rab27a enables myosin Va-dependent melanosome capture by recruiting the myosin to the organelle. J Cell Sci 114: 1091-1100

Wurmser AE, Sato TK, Emr SD (2000) New component of the vacuolar class C-Vps complex couples nucleotide exchange on the Ypt7 GTPase to SNARE-dependent docking and fusion. J Cell Biol 151: 551-562

Xu X, Kim SK (2011) The early bird catches the worm: new technologies for the Caenorhabditis elegans toolkit. Nat Rev Genet 12: 793-801

Yang H, Sasaki T, Minoshima S, Shimizu N (2007) Identification of three novel proteins (SGSM1, 2, 3) which modulate small G protein (RAP and RAB)-mediated signaling pathway. Genomics 90: 249-260

Yang J, Kim O, Wu J, Qiu Y (2002) Interaction between tyrosine kinase Etk and a RUN domain- and FYVE domain-containing protein RUFY1. A possible role of ETK in regulation of vesicle trafficking. J Biol Chem 277: 30219-30226

Yang L, Thomas ND, Helke CJ (1996) Characterization of substance P release from the intermediate area of rat thoracic spinal cord. Synapse 23: 265-273 
Yoshida H, Kitagishi Y, Okumura N, Murakami M, Nishimura Y, Matsuda S (2011) How do you RUN on? FEBS Lett 585: 1707-1710

Yu IM, Hughson FM (2010) Tethering factors as organizers of intracellular vesicular traffic. Annu Rev Cell Dev Biol 26: 137-156

Zerial M, McBride H (2001) Rab proteins as membrane organizers. Nat Rev Mol Cell Biol 2: 107-117

Zhang H, Chen J, Wang Y, Peng L, Dong X, Lu Y, Keating AE, Jiang T (2009) A computationally guided protein-interaction screen uncovers coiled-coil interactions involved in vesicular trafficking. J Mol Biol 392: 228-241

Zhang Y, Grant B, Hirsh D (2001) RME-8, a conserved J-domain protein, is required for endocytosis in Caenorhabditis elegans. Mol Biol Cell 12: 2011-2021

Zhu PC, Thureson-Klein A, Klein RL (1986) Exocytosis from large dense cored vesicles outside the active synaptic zones of terminals within the trigeminal subnucleus caudalis: a possible mechanism for neuropeptide release. Neuroscience 19: 43-54

Zimmerberg J, McLaughlin S (2004) Membrane curvature: how BAR domains bend bilayers. Curr Biol 14: R250-252 


\section{Appendix}

\section{XV.1 Materials and Reagents}

\section{XV.1.1 Laboratory equipment}

Table XV.1. Laboratory equipment used in this study

\begin{tabular}{|c|c|c|c|}
\hline Equipment & Manufacturer & Equipment & Manufacturer \\
\hline \multicolumn{2}{|c|}{ Centrifuges } & \multicolumn{2}{|c|}{ Microscopes } \\
\hline Mini-centrifuge & eppendorf & $\begin{array}{c}\text { Stereo Microscope } \\
\text { system } \\
\text { Olympus SZX9 } \\
\text { Fluorescence } \\
\text { Stereomicroscope } \\
\text { Leica MZ16 FA } \\
\text { Light source: KL2500 } \\
\text { LCD } \\
\end{array}$ & Olympus \\
\hline $\begin{array}{c}\text { Refrigerated } \\
\text { Microcentrifuge } \\
\text { Model } 5417\end{array}$ & eppendorf & \multicolumn{2}{|c|}{ Plate pouring machines } \\
\hline $\begin{array}{c}\text { Eppendorf } 5810 \mathrm{R}, \\
\text { rotor A-4-62 }\end{array}$ & eppendorf & $\begin{array}{c}\text { Plate pouring pump } \\
\text { MCP }\end{array}$ & ISMATIC \\
\hline $\begin{array}{c}\text { Sorval RC6, rotor SS- } \\
34\end{array}$ & Thermo Scientific & $\begin{array}{c}\text { Plate pouring machine } \\
\text { PourMatic MP-1000 }\end{array}$ & $\begin{array}{c}\text { New Brunswick } \\
\text { Scientific, N.Y., USA }\end{array}$ \\
\hline \multicolumn{2}{|c|}{ Electrophoresis systems } & \multicolumn{2}{|c|}{ Power supplies } \\
\hline \multirow{2}{*}{$\begin{array}{c}\text { Mini-PROTEAN }^{\circledR} 3 \\
\text { Cell electrophoresis } \\
\text { system } \\
\text { horizontal } \\
\text { electrophoresis system } \\
\text { unit }\end{array}$} & BioRad & Power supply EV231 & Consort \\
\hline & $\begin{array}{c}\text { PEQLAB } \\
\text { Biotechnologie GmbH }\end{array}$ & Power supply & Invitrogen \\
\hline Semi-Dry-Blotter & Phase & $\begin{array}{c}\text { Power supply Consort } \\
\text { EV213 } \\
\end{array}$ & Consort \\
\hline \multicolumn{2}{|c|}{ Incubators } & \multicolumn{2}{|c|}{ Shakers } \\
\hline \multirow{4}{*}{$\begin{array}{c}\text { worm incubator } \\
\text { MPR-1410 } \\
\text { Incubators } \\
\text { for } 30^{\circ} \mathrm{C} \text { and } 37^{\circ} \mathrm{C} \\
\text { Block heater SBH130 } \\
\text { Lauda EcoLine } 003 \\
\text { E100 waterbath }\end{array}$} & \multirow[t]{2}{*}{ Sanyo } & \multirow{2}{*}{$\begin{array}{c}\text { Orbital shaker for } 37^{\circ} \mathrm{C} \\
\text { Orbital shaker } \\
\text { Rotamax } 120\end{array}$} & $\begin{array}{c}\text { New Brunswick } \\
\text { Scientific, Innova40 }\end{array}$ \\
\hline & & & Heidolph, Germany \\
\hline & stuart & Thermomixer comfort & eppendorf \\
\hline & Gemini BV Laboratory & & \\
\hline
\end{tabular}




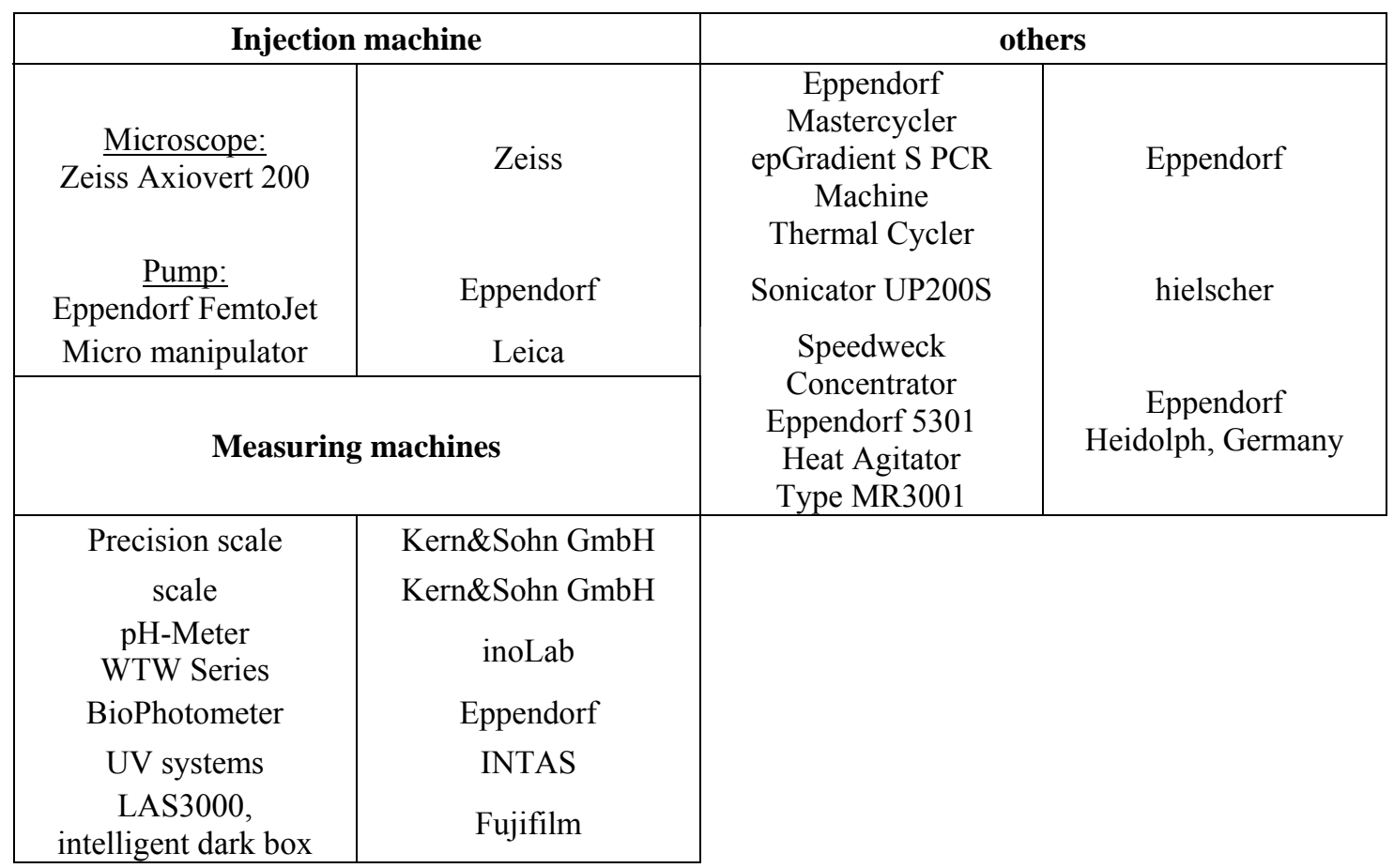

\section{XV.1.2 Chemicals}

Table XV.2. Chemicals used in this study

\begin{tabular}{|c|c|c|c|}
\hline Chemical & Manufacturer & Chemical & Manufacturer \\
\hline Adeninsulfate & AppliChem & Lithium acetate & Roth \\
\hline Agar Kobe I & AppliChem & L-Lysine & AppliChem \\
\hline Agarose & Invitrogen & Luminol & Sigma \\
\hline Alanine & AppliChem & Milk powder low fat & Rewe \\
\hline Ampicillin & Roth & Mercaptoethanol & Sigma \\
\hline Arginine & AppliChem & Methionine & AppliChem \\
\hline Asparagine & AppliChem & salmon sperm DNA & Invitrogen \\
\hline Aldicarb & Sigma & Sodium azide & Sigma \\
\hline Boric acid & Roth & Sodium chloride & Roth \\
\hline Bromphenol blue & Roth & $\begin{array}{l}\text { Sodium } \\
\text { dihydrogenphosphate }\end{array}$ & Roth \\
\hline Chloramphenicol & Roth & $\begin{array}{l}\text { di-sodium } \\
\text { hydrogenphosphate }\end{array}$ & Roth \\
\hline Cholesterol & Roth & Sodium hydroxide & Roth \\
\hline $\begin{array}{l}\text { Coomassie Brilliant } \\
\text { Blue R250 stain }\end{array}$ & BioRad & $\begin{array}{l}\text { Sodium hypochloride } \\
12 \%\end{array}$ & Roth \\
\hline Dithiothreit (DTT) & Roth & Sodium sulfate & Roth \\
\hline DMEM & PAA & $\begin{array}{l}\text { Sodium hydroxide } \\
32 \%\end{array}$ & Roth \\
\hline DMSO & Roth & Nystatine & AppliChem \\
\hline EDTA & Roth & $\begin{array}{l}\text { Prefabloc SC-Protease } \\
\text { Inhibitor }\end{array}$ & Roth \\
\hline
\end{tabular}




\begin{tabular}{|ll|ll|}
\hline Ethanol absolute $100 \%$ & Roth & Peptone & Roth \\
Ethidium bromide 1\% & Roth & Polyethylene glycol & Roth \\
Glucose & Roth & Phenylalanine & AppliChem \\
Glutamine & AppliChem & Proline & AppliChem \\
Glutamic Acid & AppliChem & Rotiphorese gel & Roth \\
Glycerol & Roth & Hydrochloric acid 32\% & Roth \\
Glutathione & Roth & Select Agar & Invitrogen \\
Yeast extract & Roth & Serine & AppliChem \\
Histidine & Sigma & Threonine & AppliChem \\
Monohydrochloride & Roth & Tris & Roth \\
IPTG & Roth & Triton X 100 & Roth \\
Imidazole & Roth & Tryptophan & Sigma \\
Immersion oil & AppliChem & Tween 20 & Roth \\
Isoleucine & Roth & Tyrosine & AppliChem \\
Isopropanol & Roth & Valine & AppliChem \\
Potassium chloride & Roth & Hydrogen peroxide & Roth \\
Kanamycin & Roth & Yeast Nitrogen Base & Invitrogen \\
LB media & Sigma & YPD Media & Roth \\
Leucine & & & \\
\hline
\end{tabular}

\section{XV.1.3 Kits/Enzymes/Reagents}

Table XV.3. Enzymes and kits used in this study

\begin{tabular}{|c|c|c|c|}
\hline \\
\hline Enzyme/Kits & Supplier & Enzyme/Kits & Supplier \\
\hline \multicolumn{2}{|c|}{ Cloning enzymes, reagents } & \multicolumn{2}{|c|}{ Markers } \\
\hline \multirow{3}{*}{$\begin{array}{l}\text { dNTP Set, } 100 \mathrm{mM} \\
\text { Solutions } \\
\text { Restriction Enzymes } \\
\text { T4 DNA Ligase }\end{array}$} & \multirow{3}{*}{$\begin{array}{l}\text { Fermentas } \\
\text { Fermentas } \\
\text { Fermentas }\end{array}$} & $\begin{array}{l}\text { GeneRuler }^{\mathrm{TM}} 1 \mathrm{~kb} \\
\text { DNA ladder } \\
\text { PageRuler }^{\mathrm{TM}}\end{array}$ & \multirow{2}{*}{$\begin{array}{l}\text { Fermentas } \\
\text { Fermentas }\end{array}$} \\
\hline & & $\begin{array}{l}\text { Prestained Protein } \\
\text { Ladder }\end{array}$ & \\
\hline & & \multicolumn{2}{|c|}{ Polymerases } \\
\hline \multicolumn{2}{|c|}{ Kits } & TaqA Polymerase & self-made, recombinant \\
\hline \multirow{2}{*}{$\begin{array}{l}\text { Mini Kit } \\
\text { Qiagen Plasmid Plus } \\
\text { Midi Kit } \\
\text { TurboFect }^{\mathrm{TM}} \text { in vitro } \\
\text { Transfection Reagent }^{\text {Transen }}\end{array}$} & \multirow{2}{*}{$\begin{array}{l}\text { Qiagen } \\
\text { Fermentas }\end{array}$} & $\begin{array}{l}\text { pTC Polymerase } \\
\text { PfuUltra II Fusion HS } \\
\text { DNA Polymerase }\end{array}$ & $\begin{array}{l}\text { self-made, recombinant } \\
\text { Stratagene }\end{array}$ \\
\hline & & \multicolumn{2}{|c|}{ Protein inhibitors } \\
\hline \multirow{2}{*}{$\begin{array}{l}\text { Gel Extraction Kits } \\
\text { PCR purification Kits }\end{array}$} & \multirow{2}{*}{$\begin{array}{l}\text { Fermentas } \\
\text { Fermentas }\end{array}$} & $\begin{array}{l}\text { Complete Mini } \\
\text { Protease Inhibitor } \\
\text { (EDTA-free) }\end{array}$ & Roche \\
\hline & & \multicolumn{2}{|c|}{ others } \\
\hline & & Proteinase K & Roth \\
\hline
\end{tabular}


XV.1.4 Antibodies

Table XV.4. Antibodies used in this study

\begin{tabular}{|c|c|c|c|c|c|}
\hline Antibody & Host & Company & $\begin{array}{l}\text { Catalog } \\
\text { Number }\end{array}$ & Application & Dilution \\
\hline \multicolumn{6}{|l|}{$\begin{array}{l}\text { Primary } \\
\text { antibodies }\end{array}$} \\
\hline $\begin{array}{l}\text { anti-gfp } \\
0.2 \mathrm{mg} / \mathrm{ml}\end{array}$ & $\begin{array}{l}\text { mouse, } \\
\text { monoclonal, } \\
\text { clone 3E6 }\end{array}$ & Invitrogen & A11120 & IP & $3 \mu \mathrm{g}$ per IP \\
\hline $\begin{array}{l}\text { anti-gfp } \\
0.4 \mathrm{mg} / \mathrm{ml}\end{array}$ & $\begin{array}{l}\text { mouse, } \\
\text { monoclonal, } \\
\text { clones } 7.1 \text { and } \\
13.1\end{array}$ & Roche & 11814460001 & WB & $1: 1000$ \\
\hline anti-V5 & $\begin{array}{l}\text { mouse, } \\
\text { monoclonal }\end{array}$ & Invitrogen & R960-25 & IP, WB & $\begin{array}{l}4 \mu \mathrm{g} \text { per IP, } \\
1: 5000\end{array}$ \\
\hline \multicolumn{6}{|l|}{$\begin{array}{l}\text { Secondary } \\
\text { antibodies }\end{array}$} \\
\hline $\begin{array}{l}\text { Peroxidase- } \\
\text { conjugated } \\
\text { AffiniPure } \\
\text { anti-mouse } \\
\text { IgG } \\
0.8 \mathrm{mg} / \mathrm{ml}\end{array}$ & goat & Jackson & & WB & $1: 10,000$ \\
\hline $\begin{array}{l}\text { Peroxidase- } \\
\text { conjugated } \\
\text { AffiniPure } \\
\text { anti- rat IgG }\end{array}$ & goat & Jackson & & WB & $1: 10,000$ \\
\hline $\begin{array}{l}\text { Peroxidase- } \\
\text { conjugated } \\
\text { AffiniPure } \\
\text { anti-rabbit }\end{array}$ & goat & Jackson & & WB & $1: 10,000$ \\
\hline
\end{tabular}

\section{XV.2 Strains and cell lines}

Table XV.5. Strains and cell lines used in this study

\begin{tabular}{|l|l|l|}
\hline Strains & Description & Source \\
\hline Bacterial strains & strain used for general molecular cloning & Invitrogen \\
\hline E. coli DH5 $\alpha$ & $\begin{array}{l}\text { strain used for molecular cloning of vectors } \\
\text { containing the } c c d \text { B gene. It contains the gyrA462 allele, } \\
\text { which confers resistance to } c c d \text { B. For gateway cloning }\end{array}$ & Invitrogen \\
\hline E. coli B121-Star (DE3) & $\begin{array}{l}\text { strain for protein expression. It contains DE3 lysogen: } \\
\text { expression of T7 polymerase is inducible by IPTG. It } \\
\text { contains additional mutation in } r n e 131 \text {, an RNAase. }\end{array}$ & Invitrogen \\
\hline $\begin{array}{l}\text { E. coli } \text { BL21- } \\
\text { CodonPlus(DE3)-RIL }\end{array}$ & $\begin{array}{l}\text { Cm } \text { r strain for protein expression; contains DE3 lysogen: } \\
\text { IPTG-inducible expression of T7 promoter controlled } \\
\text { genes; contains extra copies of the } \text { argU, ileY and leuW } \\
\text { tRNA genes }\end{array}$ & Stratagene \\
\hline
\end{tabular}




\begin{tabular}{|c|c|c|}
\hline E. coli HT115 (DE3) & $\begin{array}{l}\text { strain for feeding induced RNAi in C. elegans. } \\
\text { IPTG-inducible T7 polymerase expression, lacking the } \\
\text { dsRNA-specific RNase III }\end{array}$ & $\begin{array}{l}\text { (Kamath et } \\
\text { al, 2003; } \\
\text { Timmons et } \\
\text { al, 2001) }\end{array}$ \\
\hline E. coli $\mathrm{OP} 50$ & $\begin{array}{l}\text { strain for feeding C. elegans } \\
\text { uracil auxotroph }\end{array}$ & $\begin{array}{l}\text { (Brenner, } \\
1974)\end{array}$ \\
\hline \multicolumn{3}{|l|}{ Yeast strains } \\
\hline S. cerevisae AH109 & $\begin{array}{l}\text { strain used for yeast two-hybrid } \\
\text { MATa, trp1-901, leu2-3, 112, ura3-52, his3-200, gal4A, } \\
\text { gal80A,LYS2::GAL1 }_{\text {UAS }}-G A L 1_{T A T A}-H I S 3, \text { GAL2 } \\
\text { GALA } 2_{\text {TATA }}-A D E 2, U R A 3:: M E L 1_{U A S}-M E L 1_{\text {TATA }}-\text { lacZ }\end{array}$ & Clontech \\
\hline \multicolumn{3}{|l|}{ Cell lines } \\
\hline HEK293 & human embryonic kidney cells & ATCC \\
\hline
\end{tabular}

Table XV.6. C. elegans strains used in this study

\begin{tabular}{|c|c|}
\hline Strain name & Genotype \\
\hline CB5600 & ccIs 4251; him-8 \\
\hline GQ669 & $t b c-8(\operatorname{tm} 3802)$ \\
\hline MT1093 & unc-108(n501) \\
\hline KG1900 & unc-108(nu415) \\
\hline VC461 & $e g l-3(g k 238)$ \\
\hline RB946 & ric-19(ok833) \\
\hline RB1537 & rab-19(ok1845) \\
\hline GQ688 & rab-19(ok1845);nuIs 183[punc-129::nlp-21-venus] \\
\hline GQ584 & nuIs183[punc-129::nlp-21-venus] \\
\hline GQ026 & unc-108(n501);nuIs 183[punc-129::nlp-21-venus] \\
\hline GQ142 & unc-108(nu415);nuIs 183[punc-129::nlp-21-venus] \\
\hline GQ143 & egl-3(gk238);nuIs183[punc-129::nlp-21-venus] \\
\hline GQ641 & tbc-8(tm3802);nuls 183[punc-129::nlp-21-venus] \\
\hline GQ640 & ric-19(ok833);nuIs183[punc-129::nlp-21-venus] \\
\hline GQ642 & tbc-8(tm3802);unc-108(n501);nuIs 183[punc-129::nlp-21-venus] \\
\hline GQ643 & tbc-8(tm3802);unc-108(nu415);nuIs 183[punc-129::nlp-21-venus] \\
\hline GQ644 & tbc-8(tm3802);egl-3(gk238);nuIs183[punc-129::nlp-21-venus] \\
\hline GQ645 & tbc-8(tm3802);ric-19(ok833);nuIs183[punc-129::nlp-21-venus] \\
\hline GQ646 & tbc-8(tm3802);nuIs 183;gzEx195[prab-3::tagRFPt-tbc-8] \\
\hline GQ647 & tbc-8(tm3802);nuIs 183;gzEx196[punc-129::tagRFPt-tbc-8] \\
\hline GQ648 & tbc-8(tm3802);nuIs 183;gzEx197[punc-129::tagRFPt-tbc-8(R697A)] \\
\hline GQ649 & tbc-8(tm3802); nuIs183;gzEx198[prab-3::mcherry-rab-5(Q78L)] \\
\hline GQ585 & nuIs195[punc-129::ins-22-venus] \\
\hline GQ650 & tbc-8(tm3802);nuIs195[punc-129::ins-22-venus] \\
\hline GQ586 & ceIs $72[$ punc-129::ida-1-gfp] \\
\hline GQ651 & $t b c-8($ tm 3802);ceIs 72 [punc-129::ida-1-gfp] \\
\hline KG1645 & ceIs61[punc-129::flp-3-venus] \\
\hline GQ652 & tbc-8(tm3802);ceIs61[punc-129::flp-3-venus] \\
\hline KP3292 & nuIs 152[punc-129::gfp-snb-1] \\
\hline GQ653 & tbc-8(tm3802);nuIs 152[punc-129::gfp-snb-1] \\
\hline KP3931 & nuIs168[punc-129::venus-rab-3] \\
\hline GQ654 & tbc-8(tm3802);nuIs168[punc-129::venus-rab-3] \\
\hline Bristol & N2 (wild type) \\
\hline GQ655 & $N 2 ; g z E x 199[p t b c-8:: g f p]$ \\
\hline GQ661 & $N 2 ; g z E x 205[p r a b-3:: y f p-t b c-8 ;$ prab-3::mcherry-rab-2] \\
\hline GQ662 & N2;gzEx206[prab-3::tagRFPt-tbc-8; prab-3::manns-yfp] \\
\hline GQ663 & N2;gzEx207[prab-3::yfp-tbc-8; prab-3::mcherry-apt-9] \\
\hline
\end{tabular}




\begin{tabular}{|c|c|}
\hline GQ664 & N2;gzEx208[prab-3::yfp-tbc-8; prab-3::mcherry-rab-5] \\
\hline GQ665 & N2;gzEx209[prab-3::yfp-tbc-8; prab-3::mcherry-rab-7] \\
\hline GQ666 & N2;gzEx210[prab-3::ric-19-yfp] \\
\hline DH1336 & bIs34[prme-8::rme-8-gfp; rol-6(su1006)] \\
\hline GQ672 & tbc-8(tm3802); bIs34[prme-8::rme-8-gfp; rol-6(su1006)] \\
\hline GS1826 & 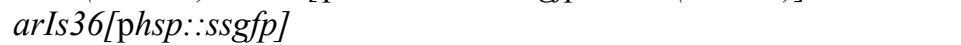 \\
\hline GQ673 & $t b c-8(t m 3802) ; \operatorname{arls} 36$ [phsp::ssgfp] \\
\hline GS2526 & $\operatorname{arIs} 37[\mathrm{pmyo-} 3: \because s s g f p]$ \\
\hline GQ674 & tbc-8(tm3802); arIs37[pmyo-3::ssgfp] \\
\hline MD701 & bcIs39[plim-7::ced-1-gfp] \\
\hline GQ675 & $t b c-8(t m 3802) ; b c I s 39[\mathrm{plim}-7: \because c e d-1-g f p]$ \\
\hline GQ676 & unc-108(nut415); bcIs39[plim-7::ced-1-gfp] \\
\hline GQ677 & eri-1(mg366);nuIs183[punc-129::nlp-21-venus] \\
\hline GQ678 & $t b c-1(\operatorname{tm} 2282)$ \\
\hline GQ679 & tbc-1(tm2282);nuIs183[punc-129::nlp-21-venus] \\
\hline XW1069 & $t b c-2(q \times 20)$ \\
\hline GQ680 & $t b c-2(q \times 20) ; n u I s 183$ [punc-129::nlp-21-venus] \\
\hline GQ681 & $t b c-4(t m 3255)$ \\
\hline GQ682 & tbc-4(tm3255);nuIs183[punc-129::nlp-21-venus] \\
\hline RB1959 & $t b c-11(o k 2576)$ \\
\hline GQ683 & tbc-11(ok2576);nuIs183[punc-129::nlp-21-venus] \\
\hline VC821 & $t b c-12(g k 362)$ \\
\hline GQ684 & tbc-12(gk362);nuIs 183[punc-129::nlp-21-venus] \\
\hline RB1516 & $t b c-13(o k 1812)$ \\
\hline GQ685 & tbc-13(ok1812);nuIs183[punc-129::nlp-21-venus] \\
\hline GQ686 & $t b c-18($ ok2374) \\
\hline GQ687 & tbc-18(ok2374);nuIs 183[punc-129::nlp-21-venus] \\
\hline GQ700 & $v p s-51(\operatorname{tm} 4275)$ \\
\hline VC625 & vps-52(ok853) \\
\hline GQ701 & vps-53(ok2864) \\
\hline VC985 & vps-54(ok1463) \\
\hline GQ689 & vps-51(tm 4275);nuIs183[punc-129::nlp-21-venus] \\
\hline GQ690 & vps-52(ok853);nuIs183[punc-129::nlp-21-venus] \\
\hline GQ691 & vps-53(ok2864);nuIs183[punc-129::nlp-21-venus] \\
\hline GQ692 & vps-54(ok1463);nuIs183[punc-129::nlp-21-venus] \\
\hline GQ702 & rund-1 $(\operatorname{tm} 3622)$ \\
\hline GQ693 & rund-1(tm3622);nuIs183[punc-129::nlp-21-venus] \\
\hline GQ694 & vps-53(ok2864);nuIs 168[punc-129::venus-rab-3] \\
\hline GQ695 & vps-52(ok853);nuIs152[punc-129::gfp-snb-1] \\
\hline GQ696 & vps-51(tm 4275);tbc-8(tm3802);nuIs 183[punc-129::nlp-21-venus] \\
\hline GQ697 & vps-51(tm 4275);rund-1(tm3622);nuIs 183[punc-129::nlp-21-venus] \\
\hline GQ698 & rund-1(tm3622);ric-19(ok833);nuIs183[punc-129::nlp-21-venus] \\
\hline GQ699 & rund-1(tm3622);tbc-8(tm3802);nuIs183[punc-129::nlp-21-venus] \\
\hline GQ145 & unc-108(n501);egl-3(gk238);nuIs183[punc-129::nlp-21-venus] \\
\hline
\end{tabular}


Table XV.7. Transgenic arrays used in this assay

\begin{tabular}{|c|c|c|}
\hline Array name & Plasmid & Markers \\
\hline$g z E x 195$ & $20 \mathrm{ng} / \mu 1 \mathrm{prab}-3: \because \operatorname{tagRFPt}-t b c-8$ & $20 \mathrm{ng} / \mu 1 p t t x-3: \because g f p$ \\
\hline$g z E x 196$ & $20 \mathrm{ng} / \mu 1$ punc-129::tagRFPt-tbc-8 & $20 \mathrm{ng} / \mu \mathrm{l} p t t x-3: \because g f p$ \\
\hline$g z E x 197$ & $20 \mathrm{ng} / \mu \mathrm{l}$ punc-129::tagRFPt-tbc-8(R697A) & $20 \mathrm{ng} / \mu \mathrm{l} p t t x-3: \because g f p$ \\
\hline$g z \operatorname{Ex} 198$ & $5 \mathrm{ng} / \mu 1$ prab-3::mcherry-rab-5(Q78L) & $20 \mathrm{ng} / \mu \mathrm{l} p t t x-3: \because g f p$ \\
\hline gzEx199 & $15 \mathrm{ng} / \mu \mathrm{l} p t b c-8:: g f p$ & $40 \mathrm{ng} / \mu 1$ pRF4-rol-6(su1006) \\
\hline$g z E x 205$ & $\begin{array}{l}20 \mathrm{ng} / \mu \mathrm{l} \text { prab-3::yfp-tbc- } 8 \\
5 \mathrm{ng} / \mu \mathrm{l} \text { prab-3::mcherry-rab-2}\end{array}$ & $\begin{array}{l}40 \mathrm{ng} / \mu \mathrm{l} \text { pRF4-rol-6(su1006) } \\
20 \mathrm{ng} / \mu \mathrm{l} \text { pttx-3:.gfp }\end{array}$ \\
\hline$g z \operatorname{Ex} 206$ & $\begin{array}{l}20 \mathrm{ng} / \mu 1 \text { prab-3::tagRFPt-tbc-8; } \\
10 \mathrm{ng} / \mu \mathrm{l} \text { prab-3::manns-yfp }\end{array}$ & $\begin{array}{l}40 \mathrm{ng} / \mu \mathrm{l} \text { pRF4-rol-6(su1006) } \\
20 \mathrm{ng} / \mu \mathrm{l} \text { pttx-3::gfp }\end{array}$ \\
\hline$g z E x 207$ & 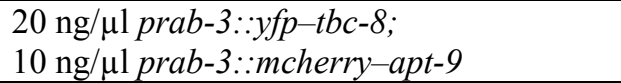 & $\begin{array}{l}40 \mathrm{ng} / \mu 1 \text { pRF4-rol-6(su1006) } \\
20 \mathrm{ng} / \mu 1 \text { pttx-3::gfp }\end{array}$ \\
\hline$g z \operatorname{Ex} 208$ & $\begin{array}{l}20 \mathrm{ng} / \mu 1 \text { prab-3::yfp-tbc-8; } \\
5 \mathrm{ng} / \mu \mathrm{l} \text { prab-3::mcherry-rab-5}\end{array}$ & $\begin{array}{l}40 \mathrm{ng} / \mu \mathrm{l} \text { pRF4-rol-6(su1006) } \\
20 \mathrm{ng} / \mu \mathrm{l} \text { pttx-3::gfp }\end{array}$ \\
\hline$g z E x 209$ & $\begin{array}{l}20 \mathrm{ng} / \mu 1 \text { prab-3::yfp-tbc- } 8 ; \\
5 \mathrm{ng} / \mu 1 \text { prab-3::mcherry-rab-7 }\end{array}$ & $\begin{array}{l}40 \mathrm{ng} / \mu \mathrm{l} \text { pRF4-rol-6(su1006) } \\
20 \mathrm{ng} / \mu \mathrm{pttx}-3: \because g f p\end{array}$ \\
\hline$g z \operatorname{Ex} 210$ & $20 \mathrm{ng} / \mu \mathrm{l}$ prab-3::ric-19-yfp & $\begin{array}{l}40 \mathrm{ng} / \mu 1 \text { pRF4-rol-6(su1006) } \\
20 \mathrm{ng} / \mu 1 \text { pttx-3::gfp } \\
20 \mathrm{ng} / \mu \mathrm{l} \text { punc-129::tagRFP }\end{array}$ \\
\hline
\end{tabular}

\section{XV.3 Oligonucleotides}

Table XV.8. DNA sequences of primers used in this study

\begin{tabular}{|c|c|}
\hline Primer number & Primer sequence \\
\hline oGQ315 & ggatgtgttcacagttcctgccaatgatgattt \\
\hline oGQ316 & ctattcaaacataatgtcattaagcgactcgag \\
\hline oGQ318 & gagtggaataccatccttccttgtcacacttgg \\
\hline oGQ591 & cccgaattctcaagtgtactgacaacatcg \\
\hline oGQ592 & gggggtaccgccatggctgcacagttttacgaaaggaacaca \\
\hline oGQ601 & gttttgcccaacgattgtct \\
\hline oGQ983 & gctggaccggtaggatcccatatgtctagtgttttggacgtc \\
\hline oGQ1036 & aatacgactcactataggttcacgtttcacttcgcgacgggcttccagat \\
\hline oGQ1037 & gtgccactgggttgcgaattc \\
\hline oGQ1249 & ggtatttcgaattcaggcaaccacgtg \\
\hline oGQ1250 & aagtcttccaggccattgccaacatga \\
\hline oGQ1251 & aattatctgtttacagtagatttatga \\
\hline oGQ1278 & tcgtaacttccttcactacgetc \\
\hline oGQ1280 & ccatcctcgacgaaacgetc \\
\hline oGQ1283 & cttgattccgtttgcctcattg \\
\hline oGQ1295 & gggcacactactttcaacactc \\
\hline oGQ1297 & gtgtactgacaacatcgttgc \\
\hline oGQ1298 & gcagctccgaatgttgtgaag \\
\hline oGQ1542 & gaactgttggaacgtctttatactgcgat \\
\hline oGQ1543 & gaaatgaaatctctcagccgaagtatgtcg \\
\hline oGQ1544 & cttcaaaaccgtaataagatcttctcgttt \\
\hline oGQ1560 & tgatcctccactttgggttg \\
\hline oGQ1561 & cctctccagtagacccgttt \\
\hline oGQ1562 & gtttggcagagagacgctgaa \\
\hline oGQ1622 & ttcccgggttaccggttatgtggagggcgaagaagccaaca \\
\hline oGQ1623 & ggggcggccgccetcgagctacttgaggtgttgcacaaggtt \\
\hline oGQ1654 & ccaagaaagtatcaaaaacactccaaatag \\
\hline
\end{tabular}


oGQ1656

oGQ1657

oGQ1666

oGQ1667

oGQ1698

oGQ1699

oGQ1735

oGQ1791

oGQ1793

oGQ1800

oGQ1801

oGQ1802

oGQ1843

oGQ2055

oGQ2144

oGQ2145

oGQ2230

oGQ2231

oGQ2462

oGQ2353

oSE227

oSE228

oSE270 atcaatgcatcaggttcactgctcaacttg

cgtatcgtagataccttcataatagagtta

tgaagctagcacggtacccatggaaagatctatgatgaacgagttggaa

cctactcgagtcagcggecgctaaatgcgtctttgatttg

gacgtggaggcatgcgatagaaatttgatgttc

tctatcgcatgectccacgtcettgtcaattct

cccaccatggatccgatgagcatgggatcaacgecgetgtctggt

cccgcggtaccggtaatgattaatacaaaagaagtccgaaga

cttaagcttctgcaggaacttttccatctg

gggctcgagctagcggcegccgggtacccattttagaga

ttccegggttaccggttatgaatcaacttatgcacgcgag

ttccegggttaccggttatgegtttggtaagaacttctggtg

cccetcgagctagcggecgccatcttgegtgagtcg

agtaaccggtgccetccacatatctgccgatgaatgccg

ttccegggttaccggtttccatggaagaagacgtagtg

ggggcggcegecctcgagtcaattatcctgagttgt

ttaagtggaatcatcgggtgc

cttgegtgagtcgagatgatg

getttcggacaatagttaccac

gggctcgagctagcggccgectcttcggacttctttgtattaat

cagcttttgagcacaactgac

tggctcagctcgactctaact

cccaccggtatccatgggaatgcctcgaacacgtgtcaac

Table XV.9. Deletion alleles with respective worm PCR primers used in this study

\begin{tabular}{|c|c|c|c|c|c|c|}
\hline $\begin{array}{l}\text { Deletion } \\
\text { strain }\end{array}$ & $\begin{array}{l}\text { Size of } \\
\text { deletion } \\
\text { (bp) }\end{array}$ & $\begin{array}{l}\text { Outside deletion } \\
\text { primer pair }\end{array}$ & $\begin{array}{l}\text { PCR } \\
\text { band } \\
\text { size } \\
\text { in } \\
\text { WT } \\
\text { (bp) } \\
\end{array}$ & $\begin{array}{l}\text { Inside deletion } \\
\text { primer pair }\end{array}$ & $\begin{array}{l}\text { PCR } \\
\text { band } \\
\text { size } \\
\text { in } \\
\text { WT } \\
\text { (bp) }\end{array}$ & $\begin{array}{l}\text { Anneal. } \\
\text { temp/ } \\
\text { Extens. } \\
\text { time }\end{array}$ \\
\hline $\begin{array}{l}t b c-8 \\
(t m 3802)\end{array}$ & 378 & oGQ1560/oGQ1561 & 1100 & oGQ1562/oGQ1560 & 666 & $\begin{array}{l}51^{\circ} \mathrm{C} / \\
1: 10 \mathrm{~min}\end{array}$ \\
\hline $\begin{array}{l}\text { unc-108 } \\
\text { (nu415) }\end{array}$ & 201 & oGQ1278/oGQ1280 & 1755 & oGQ1278/oGQ1283 & 1179 & $\begin{array}{l}58^{\circ} \mathrm{C} / \\
2 \mathrm{~min}\end{array}$ \\
\hline $\begin{array}{l}\text { egl-3 } \\
(g k 238)\end{array}$ & 1460 & oGQ1249/oGQ1250 & 2030 & oGQ1251/oGQ1250 & 1000 & $\begin{array}{l}55^{\circ} \mathrm{C} / \\
2: 10 \mathrm{~min}\end{array}$ \\
\hline $\begin{array}{l}r a b-19 \\
(o k 1845)\end{array}$ & 1257 & oGQ1295/oGQ591 & 2000 & oGQ1298/oGQ1297 & 1300 & $\begin{array}{l}52^{\circ} \mathrm{C} / \\
2: 10 \mathrm{~min}\end{array}$ \\
\hline $\begin{array}{l}\text { ric-19 } \\
\text { (ok833) }\end{array}$ & 1671 & oGQ592/oGQ601 & 3300 & oGQ592/oGQ603 & 2000 & $\begin{array}{l}57^{\circ} \mathrm{C} / \\
3: 30 \mathrm{~min}\end{array}$ \\
\hline $\begin{array}{l}\text { rund-1 } \\
\text { (tm3622) }\end{array}$ & 577 & oGQ2230/oGQ2231 & 1140 & oGQ2230/oGQ2462 & 600 & $\begin{array}{l}55^{\circ} \mathrm{C} / \\
1: 30 \mathrm{~min}\end{array}$ \\
\hline $\begin{array}{l}\text { vps-51 } \\
(\operatorname{tm} 4275)\end{array}$ & 267 & oGQ1544/oGQ983 & 980 & oGQ1543/oGQ1542 & 315 & $\begin{array}{l}45^{\circ} \mathrm{C} / \\
1: 30 \mathrm{~min}\end{array}$ \\
\hline $\begin{array}{l}v p s-52 \\
\text { (ok853) }\end{array}$ & 902 & oSE270/oSE227 & 1600 & oSE270/oSE228 & 560 & $\begin{array}{l}47^{\circ} \mathrm{C} / \\
2: 30 \mathrm{~min}\end{array}$ \\
\hline $\begin{array}{l}\text { vps- } \\
53(\text { ok2864) }\end{array}$ & 373 & oGQ1656/oGQ1657 & 1060 & oGQ1654/oGQ1657 & 855 & $\begin{array}{l}51^{\circ} \mathrm{C} / \\
1: 30 \mathrm{~min}\end{array}$ \\
\hline $\begin{array}{l}p s- \\
54(\text { ok1463) }\end{array}$ & 739 & oGQ315/oGQ316 & 2600 & oGQ315/oGQ318 & 1400 & $\begin{array}{l}54^{\circ} \mathrm{C} / \\
3: 00 \mathrm{~min}\end{array}$ \\
\hline $\begin{array}{l}\text { unc- } \\
108(n 501)\end{array}$ & $\mathrm{g} \rightarrow \mathrm{a}$ & oGQ1036/oGQ1037 & \multicolumn{3}{|c|}{ 2x PCR, digestion with Sau3AI } & $\begin{array}{l}54^{\circ} \mathrm{C} / \\
0.5 \mathrm{~min}\end{array}$ \\
\hline
\end{tabular}




\section{XV.4 Plasmids}

Table XV.10. Plasmids used in this study

\begin{tabular}{|c|c|c|c|}
\hline Backbone Vectors & Resistance & Description & Source \\
\hline $\begin{array}{l}\text { pPD115.62(pmyo- } \\
3: \because g f p)\end{array}$ & $A m p^{r}$ & $\begin{array}{l}\text { Muscle expression vector for } C \text {. } \\
\text { elegans. Contains } u n c-543 U ́ T R\end{array}$ & $\begin{array}{l}\text { Andrew Fire } \\
\text { vector kit }\end{array}$ \\
\hline prab-3::mcherry & $\mathrm{Amp}^{\mathrm{r}}$ & $\begin{array}{l}\text { Neuronal expression vector } \\
\text { for C. elegans. Based on pPD } 115.62\end{array}$ & $\begin{array}{l}\text { Kindly provided by } \\
\text { Marija Sumakovic }\end{array}$ \\
\hline prab-3::tagRFPt & $\mathrm{Amp}^{\mathrm{r}}$ & $\begin{array}{l}\text { Neuronal expression vector } \\
\text { for C. elegans. Based on pPD115.62 }\end{array}$ & Cloned in this study \\
\hline prab-3::yfp & $\mathrm{Amp}^{\mathrm{r}}$ & $\begin{array}{l}\text { Neuronal expression vector } \\
\text { for C. elegans. Based on pPD1 } 15.62\end{array}$ & $\begin{array}{l}\text { Kindly provided by } \\
\text { Marija Sumakovic }\end{array}$ \\
\hline punc-129::tagRFPt & $A m p^{r}$ & $\begin{array}{l}\text { DA and DB motoneuron expression } \\
\text { vector for } C \text {. elegans. } \\
\text { Based on prab-3::mcherry }\end{array}$ & Cloned in this study \\
\hline L4440 & $\mathrm{Amp}^{\mathrm{r}}$ & Entry vector for feeding RNAi & $\begin{array}{l}\text { Kindly provided by } \\
\text { Julie Ahringer }\end{array}$ \\
\hline pFASTBAC m30b & $\mathrm{Amp}^{\mathrm{r}}$ & $\begin{array}{l}\text { GST-Protein expression vector in } \\
\text { insect cells }\end{array}$ & Invitrogen \\
\hline pFASTBAC HTc & $A m p^{r}$ & 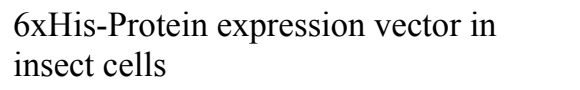 & Invitrogen \\
\hline pGADT7 & $\mathrm{Amp}^{\mathrm{r}}$ & $\begin{array}{l}\text { Yeast-2-hybrid vector containing } \\
\text { DNA activating domain }\end{array}$ & Clontech \\
\hline pGBKT7 & $\mathrm{Kan}^{\mathrm{r}}$ & $\begin{array}{l}\text { Yeast-2-hybrid vector containing } \\
\text { DNA binding domain }\end{array}$ & Clontech \\
\hline pGST parallel 2 & $A m p^{r}$ & $\begin{array}{l}\text { GST-Protein expression vector based } \\
\text { on pGEX } 4 \mathrm{~T} 1\end{array}$ & (Sheffield et al, 1999) \\
\hline pHis parallel 2 & $A m p^{r}$ & $\begin{array}{l}\text { His-tag protein expression vector based } \\
\text { on pGEX4T1 }\end{array}$ & (Sheffield et al, 1999) \\
\hline $\begin{array}{l}\text { pcDNA3.1 nV5- } \\
\text { DEST }\end{array}$ & $\mathrm{Amp}^{\mathrm{r}}$ & $\begin{array}{l}\text { V5-Protein expression vector in } \\
\text { (mammalian) cell culture }\end{array}$ & $\begin{array}{l}\text { Kindly provided by } \\
\text { Dr. John Chua }\end{array}$ \\
\hline $\begin{array}{l}\text { pcDNA6.2 N- } \\
\text { EmGFP-DEST }\end{array}$ & $\mathrm{Amp}^{\mathrm{r}}$ & $\begin{array}{l}\text { N-terminal EmGFP expression vector } \\
\text { in (mammalian) cell culture }\end{array}$ & $\begin{array}{l}\text { Kindly provided by } \\
\text { Dr. John Chua }\end{array}$ \\
\hline $\begin{array}{l}\text { Genes of interest } \\
\text { in RNAi vectors }\end{array}$ & Resistance & Cloning Strategy & Source \\
\hline L4440 tbc- 8 & $A m p^{r}$ & $\begin{array}{l}\text { PCR with oGQ1622/oGQ1623 } \\
\text { Digested with AgeI/XhoI }\end{array}$ & Cloned in this study \\
\hline $\begin{array}{l}\text { Genes of interest } \\
\text { in protein } \\
\text { expression vectors }\end{array}$ & Resistance & Cloning Strategy & Source \\
\hline $\begin{array}{l}\text { pGST parallel } 2 t b c- \\
8 \text { small } 587-903 \text { aa }\end{array}$ & $A m p^{r}$ & $\begin{array}{l}\text { Subcloned from pGADT7 tbc-8 small } \\
\text { Digested with AgeI/XhoI }\end{array}$ & Cloned in this study \\
\hline $\begin{array}{l}\text { pGST parallel } 2 \text { tbc- } \\
8 \text { medium } \\
451-903 \text { aa }\end{array}$ & $\mathrm{Amp}^{\mathrm{r}}$ & $\begin{array}{l}\text { Subcloned from pGADT } 7 t b c-8 \\
\text { medium; Digested with AgeI/XhoI }\end{array}$ & Cloned in this study \\
\hline $\begin{array}{l}\text { pGST parallel } 2 t b c- \\
8 \text { large } 329-903 \text { aa }\end{array}$ & $A m p^{r}$ & $\begin{array}{l}\text { Subcloned from pGADT } 7 b c-8 \text { large } \\
\text { Digested with AgeI/XhoI }\end{array}$ & Cloned in this study \\
\hline $\begin{array}{l}\text { pGST parallel } 2 t b c- \\
8 \text { full length } \\
1-903 \text { aa }\end{array}$ & $\mathrm{Amp}^{\mathrm{r}}$ & $\begin{array}{l}\text { Subcloned from pGADT7 tbc- } 8 \\
\text { Digested with AgeI/XhoI }\end{array}$ & Cloned in this study \\
\hline $\begin{array}{l}\text { pFASTBAC m30b } \\
t b c-8\end{array}$ & $A m p^{r}$ & $\begin{array}{l}\text { Subcloned from GST parallel } t b c-8 \\
\text { Digested with NcoI/XhoI }\end{array}$ & Cloned in this study \\
\hline $\begin{array}{l}\text { pFASTBAC m30b } \\
\text { tbc- } 8 \text { small }\end{array}$ & $A m p^{r}$ & $\begin{array}{l}\text { Subcloned from GST parallel } t b c-8 \\
\text { small; Digested with NcoI/NotI }\end{array}$ & Cloned in this study \\
\hline
\end{tabular}




\begin{tabular}{|c|c|c|c|}
\hline $\begin{array}{l}\text { pFASTBAC m } 30 \mathrm{~b} \\
\text { tbc- } 8 \text { codon } \\
\text { optimized }\end{array}$ & $A m p^{r}$ & $\begin{array}{l}\text { Subcloned from Clontech vector } \\
\text { Digested with NcoI/XhoI }\end{array}$ & Cloned in this study \\
\hline $\begin{array}{l}\text { pFASTBAC HTc } \\
\text { tbc- } 8\end{array}$ & $A m p^{r}$ & $\begin{array}{l}\text { Subcloned from GST parallel } t b c-8 \\
\text { Digested with NcoI/XhoI }\end{array}$ & Cloned in this study \\
\hline $\begin{array}{l}\text { pFASTBAC HTc } \\
\text { tbc- } 8 \text { small }\end{array}$ & $A m p^{r}$ & $\begin{array}{l}\text { Subcloned from GST parallel } t b c-8 \\
\text { small; Digested with NcoI/NotI }\end{array}$ & Cloned in this study \\
\hline $\begin{array}{l}\text { pFASTBAC HTc } \\
\text { tbc- } 8 \text { codon } \\
\text { optimized }\end{array}$ & $A m p^{r}$ & $\begin{array}{l}\text { Subcloned from Clontech vector } \\
\text { Digested with NcoI/XhoI }\end{array}$ & Cloned in this study \\
\hline $\begin{array}{l}\text { Genes of interest } \\
\text { in mammalian } \\
\text { expression vectors }\end{array}$ & Resistance & Cloning Strategy & Source \\
\hline $\begin{array}{l}\text { pcDNA3.1 } \\
n V 5-t b c-8\end{array}$ & $A m p^{r}$ & Gateway cloning & Cloned in this study \\
\hline $\begin{array}{l}\text { pcDNA3.1 } \\
n V 5-t b c-81-597 \mathrm{aa}\end{array}$ & $\mathrm{Amp}^{\mathrm{r}}$ & Gateway cloning & Cloned in this study \\
\hline $\begin{array}{l}\text { pcDNA3.1 } \\
\text { nV5-rund-1 }\end{array}$ & $\mathrm{Amp}^{\mathrm{r}}$ & Gateway cloning & Cloned in this study \\
\hline $\begin{array}{l}\text { pcDNA3.1 } \\
n V 5-v p s-51\end{array}$ & $A m p^{r}$ & Gateway cloning & Cloned in this study \\
\hline $\begin{array}{l}\text { pcDNA6.2 N- } \\
\text { EmGFP-ric-19 }\end{array}$ & $A m p^{r}$ & Gateway cloning & Cloned in this study \\
\hline $\begin{array}{l}\text { pcDNA6.2 N- } \\
\text { EmGFP-tbc-8 }\end{array}$ & $A m p^{r}$ & Gateway cloning & Cloned in this study \\
\hline $\begin{array}{l}\text { pcDNA6.2 N- } \\
\text { EmGFP-rund-1 }\end{array}$ & $\mathrm{Amp}^{\mathrm{r}}$ & Gateway cloning & Cloned in this study \\
\hline $\begin{array}{l}\text { pcDNA6.2 N- } \\
\text { EmGFP-vps-51 }\end{array}$ & $A m p^{r}$ & Gateway cloning & Cloned in this study \\
\hline $\begin{array}{l}\text { Genes of interest } \\
\text { in Y2H vectors }\end{array}$ & Resistance & Cloning Strategy & Source \\
\hline $\begin{array}{l}\text { pGBKT7 rab-1 } \\
(Q 70 L)\end{array}$ & $\operatorname{Kan}^{\mathrm{r}}$ & -- & $\begin{array}{l}\text { Kindly provided by } \\
\text { Katrin Schwarze }\end{array}$ \\
\hline $\begin{array}{l}\text { pGBKT7 } r a b-2 \\
(Q 65 L)\end{array}$ & $\operatorname{Kan}^{\mathrm{r}}$ & -- & $\begin{array}{l}\text { Kindly provided by } \\
\text { Marija Sumakovic }\end{array}$ \\
\hline $\begin{array}{l}\text { pGBKT7 } r a b-3 \\
(Q 81 L)\end{array}$ & $\operatorname{Kan}^{\mathrm{r}}$ & -- & $\begin{array}{l}\text { Kindly provided by } \\
\text { Nikhil Sasidharan }\end{array}$ \\
\hline $\begin{array}{l}\text { pGBKT7 rab-5 } \\
(Q 78 L)\end{array}$ & $\operatorname{Kan}^{\mathrm{r}}$ & -- & $\begin{array}{l}\text { Kindly provided by } \\
\text { Nikhil Sasidharan }\end{array}$ \\
\hline $\begin{array}{l}\text { pGBKT7 rab-6.1 } \\
(Q 70 L)\end{array}$ & $\operatorname{Kan}^{\mathrm{r}}$ & -- & $\begin{array}{l}\text { Kindly provided by } \\
\text { Katrin Schwarze }\end{array}$ \\
\hline $\begin{array}{l}\text { pGBKT7 rab-6.2 } \\
(Q 69 L)\end{array}$ & $\operatorname{Kan}^{\mathrm{r}}$ & -- & $\begin{array}{l}\text { Kindly provided by } \\
\text { Katrin Schwarze }\end{array}$ \\
\hline $\begin{array}{l}\text { pGBKT7 } r a b-7 \\
(Q 68 L)\end{array}$ & $\operatorname{Kan}^{\mathrm{r}}$ & -- & $\begin{array}{l}\text { Kindly provided by } \\
\text { Nikhil Sasidharan }\end{array}$ \\
\hline $\begin{array}{l}\text { pGBKT7 } r a b-8 \\
(Q 67 L)\end{array}$ & $\operatorname{Kan}^{\mathrm{r}}$ & -- & $\begin{array}{l}\text { Kindly provided by } \\
\text { Nikhil Sasidharan }\end{array}$ \\
\hline $\begin{array}{l}\text { pGBKT7 } r a b-10 \\
(Q 68 L)\end{array}$ & $\mathrm{Kan}^{\mathrm{r}}$ & -- & $\begin{array}{l}\text { Kindly provided by } \\
\text { Nikhil Sasidharan }\end{array}$ \\
\hline $\begin{array}{l}\text { pGBKT7 } r a b-11.1 \\
(Q 70 L)\end{array}$ & $\operatorname{Kan}^{r}$ & -- & $\begin{array}{l}\text { Kindly provided by } \\
\text { Nikhil Sasidharan }\end{array}$ \\
\hline $\begin{array}{l}\text { pGBKT7 } r a b-14 \\
(Q 70 L)\end{array}$ & $\operatorname{Kan}^{\mathrm{r}}$ & -- & $\begin{array}{l}\text { Kindly provided by } \\
\text { Nikhil Sasidharan }\end{array}$ \\
\hline $\begin{array}{l}\text { pGBKT7 rab-18 } \\
(Q 70 L)\end{array}$ & $\operatorname{Kan}^{r}$ & -- & $\begin{array}{l}\text { Kindly provided by } \\
\text { Nikhil Sasidharan }\end{array}$ \\
\hline $\begin{array}{l}\text { pGBKT7 rab-19 } \\
(Q 69 L)\end{array}$ & $\operatorname{Kan}^{\mathrm{r}}$ & -- & $\begin{array}{l}\text { Kindly provided by } \\
\text { Nikhil Sasidharan }\end{array}$ \\
\hline
\end{tabular}




\begin{tabular}{|c|c|}
\hline $\begin{array}{l}\text { pGBKT7 rab-21 } \\
(Q 71 L)\end{array}$ & $\operatorname{Kan}^{r}$ \\
\hline $\begin{array}{l}\text { pGBKT7 rab-28 } \\
(Q 95 L)\end{array}$ & $\operatorname{Kan}^{\mathrm{r}}$ \\
\hline $\begin{array}{l}\text { pGBKT7 rab-30 } \\
(Q 66 L)\end{array}$ & $\operatorname{Kan}^{r}$ \\
\hline $\begin{array}{l}\text { pGBKT7 rab-33 } \\
(Q 159 L)\end{array}$ & $\operatorname{Kan}^{r}$ \\
\hline $\begin{array}{l}\text { pGBKT7 rab-35 } \\
(Q 69 L)\end{array}$ & $\operatorname{Kan}^{r}$ \\
\hline $\begin{array}{l}\text { pGBKT7 rab-37.1 } \\
(Q 37 L)\end{array}$ & $\operatorname{Kan}^{r}$ \\
\hline $\begin{array}{l}\text { pGBKT7 rab-37.2 } \\
(Q 89 L)\end{array}$ & $\operatorname{Kan}^{r}$ \\
\hline $\begin{array}{l}\text { pGBKT7 } r a b-39 \\
(Q 77 L)\end{array}$ & $\operatorname{Kan}^{r}$ \\
\hline $\begin{array}{l}\text { pGBKT7 } r a b-2 \\
(S 20 N)\end{array}$ & $\operatorname{Kan}^{r}$ \\
\hline $\begin{array}{l}\text { pGBKT7 rab-14 } \\
(S 25 N)\end{array}$ & $\operatorname{Kan}^{\mathrm{r}}$ \\
\hline $\begin{array}{l}\text { pGBKT7 rab-19 } \\
(T 24 N)\end{array}$ & $\operatorname{Kan}^{r}$ \\
\hline GBKT7 $r a b-2 \mathrm{wt}$ & $\operatorname{Kan}^{\mathrm{r}}$ \\
\hline
\end{tabular}

\begin{tabular}{|c|c|c|}
\hline pGBKT7 tbc-8 & $\operatorname{Kan}^{r}$ & $\begin{array}{l}\text { PCR with oGQ1622/oGQ1623 } \\
\text { Digested with AgeI/XhoI }\end{array}$ \\
\hline $\begin{array}{l}\mathrm{pGBKT} 7 t b c-8 \\
(R 697 A)\end{array}$ & $\operatorname{Kan}^{r}$ & $\begin{array}{l}\text { Mutagenesis PCR with } \\
\text { oGQ1698/oGQ1699 }\end{array}$ \\
\hline $\begin{array}{l}\text { pGBKT7 tbc-8 } \\
1-597 \text { aa }\end{array}$ & $\operatorname{Kan}^{r}$ & $\begin{array}{l}\text { PCR with oGQ1622/oGQ2353 } \\
\text { Digested with AgeI/XhoI }\end{array}$ \\
\hline $\begin{array}{l}\text { pGBKT7 tbc-8 } \\
586-903 \text { aа }\end{array}$ & $\operatorname{Kan}^{r}$ & $\begin{array}{l}\text { PCR with oGQ1791/oGQ1623 } \\
\text { Digested with AgeI/XhoI }\end{array}$ \\
\hline $\begin{array}{l}\text { pGBKT7 tbc-8 } \\
451-903 \text { aa }\end{array}$ & $\operatorname{Kan}^{r}$ & $\begin{array}{l}\text { PCR with oGQ1802/oGQ1623 } \\
\text { Digested with AgeI/XhoI }\end{array}$ \\
\hline $\begin{array}{l}\text { pGBKT7 tbc-8 } \\
329-903 \text { aа }\end{array}$ & $\operatorname{Kan}^{r}$ & $\begin{array}{l}\text { PCR with oGQ1801/oGQ1623 } \\
\text { Digested with AgeI/XhoI }\end{array}$ \\
\hline $\begin{array}{l}\text { pGBKT7 tbc-8 } \\
1-328 \text { aа }\end{array}$ & $\operatorname{Kan}^{r}$ & $\begin{array}{l}\text { PCR with oGQ1622/oGQ2353 } \\
\text { Digested with AgeI/XhoI }\end{array}$ \\
\hline $\begin{array}{l}\text { pGBKT7 tbc-8 } \\
329-597 \text { aa }\end{array}$ & $\operatorname{Kan}^{r}$ & $\begin{array}{l}\text { PCR with oGQ1801/oGQ1800 } \\
\text { Digested with AgeI/XhoI }\end{array}$ \\
\hline pGBKT7 rap-1 & $\operatorname{Kan}^{\mathrm{r}}$ & -- \\
\hline pGBKT7 rap-2 & $\mathrm{Kan}^{\mathrm{r}}$ & -- \\
\hline pGBKT7 rap-3 & $\operatorname{Kan}^{r}$ & -- \\
\hline pGBKT7 ral-1 & $\operatorname{Kan}^{\mathrm{r}}$ & -- \\
\hline pGBKT7 ras-1 & $\operatorname{Kan}^{\mathrm{r}}$ & -- \\
\hline $\begin{array}{l}\text { pGBKT7 rap-1 } \\
(G 12 \mathrm{~V})\end{array}$ & $\operatorname{Kan}^{r}$ & -- \\
\hline $\begin{array}{l}\text { pGBKT7 rap }-2 \\
(G 12 \mathrm{~V})\end{array}$ & $\operatorname{Kan}^{r}$ & -- \\
\hline
\end{tabular}

Kindly provided by Nikhil Sasidharan

Kindly provided by Nikhil Sasidharan

Kindly provided by Nikhil Sasidharan

Kindly provided by Nikhil Sasidharan Kindly provided by Nikhil Sasidharan

Kindly provided by Nikhil Sasidharan

Kindly provided by Nikhil Sasidharan

Kindly provided by Nikhil Sasidharan

Kindly provided by Marija Sumakovic

Kindly provided by Nikhil Sasidharan

Kindly provided by Nikhil Sasidharan

Kindly provided by Marija Sumakovic

Cloned in this study

Cloned in this study

Cloned in this study

Cloned in this study

Cloned in this study

Cloned in this study

Cloned in this study

Cloned in this study

Kindly provided by Sabine König

Kindly provided by Sabine König

Kindly provided by Sabine König

Kindly provided by Sabine König

Kindly provided by Sabine König

Kindly provided by Sabine König

Kindly provided by Sabine König 


\begin{tabular}{|c|c|c|c|}
\hline $\begin{array}{l}\text { pGBKT7 rap-3 } \\
(G 12 \mathrm{~V})\end{array}$ & $\operatorname{Kan}^{r}$ & -- & $\begin{array}{l}\text { Kindly provided by } \\
\text { Sabine König }\end{array}$ \\
\hline $\begin{array}{l}\text { pGBKT7 ral-1 } \\
(G 26 V)\end{array}$ & $\operatorname{Kan}^{r}$ & -- & $\begin{array}{l}\text { Kindly provided by } \\
\text { Sabine König }\end{array}$ \\
\hline $\begin{array}{l}\text { pGBKT7 ras-1 } \\
(G 26 V)\end{array}$ & $\operatorname{Kan}^{\mathrm{r}}$ & -- & $\begin{array}{l}\text { Kindly provided by } \\
\text { Sabine König }\end{array}$ \\
\hline pGBKT7 vps-51 & $\operatorname{Kan}^{\mathrm{r}}$ & -- & (Luo et al, 2011) \\
\hline pGBKT7 vps-52 & $\operatorname{Kan}^{\mathrm{r}}$ & -- & (Luo et al, 2011) \\
\hline pGBKT7 vps-53 & $\operatorname{Kan}^{\mathrm{r}}$ & -- & (Luo et al, 2011) \\
\hline pGBKT7 vps-54 & $\operatorname{Kan}^{\mathrm{r}}$ & -- & (Luo et al, 2011) \\
\hline pGADT7 tbc-8 & $\mathrm{Amp}^{\mathrm{r}}$ & $\begin{array}{l}\text { PCR with oGQ1622/oGQ1623 } \\
\text { Digested with AgeI/XhoI }\end{array}$ & Cloned in this study \\
\hline $\begin{array}{l}\text { pGADT7 tbc-8 } \\
(\text { R697A) }\end{array}$ & $A m p^{r}$ & $\begin{array}{l}\text { Mutagenesis PCR with } \\
\text { oGQ1698/oGQ1699 }\end{array}$ & Cloned in this study \\
\hline $\begin{array}{l}\text { pGADT7 tbc-8 } \\
1-597 \text { aa }\end{array}$ & $A m p^{r}$ & $\begin{array}{l}\text { PCR with oGQ1622/oGQ2353 } \\
\text { Digested with AgeI/XhoI }\end{array}$ & Cloned in this study \\
\hline pGADT7 ric-19 & $A m p^{r}$ & -- & $\begin{array}{l}\text { Kindly provided by } \\
\text { Marija Sumakovic }\end{array}$ \\
\hline pGADT7 ica69 & $A m p^{r}$ & -- & $\begin{array}{l}\text { Kindly provided by } \\
\text { Marija Sumakovic }\end{array}$ \\
\hline $\begin{array}{l}\text { pGADT7 } t b c-8 \\
586-903 \text { aа }\end{array}$ & $A m p^{r}$ & $\begin{array}{l}\text { PCR with oGQ1791/oGQ1623 } \\
\text { Digested with AgeI/XhoI }\end{array}$ & Cloned in this study \\
\hline $\begin{array}{l}\text { pGADT7 } t b c-8 \\
451-903 \text { aа }\end{array}$ & $\mathrm{Amp}^{\mathrm{r}}$ & $\begin{array}{l}\text { PCR with oGQ1802/oGQ1623 } \\
\text { Digested with AgeI/XhoI }\end{array}$ & Cloned in this study \\
\hline $\begin{array}{l}\text { pGADT7 } t b c-8 \\
329-903 \text { aa }\end{array}$ & $A m p^{r}$ & $\begin{array}{l}\text { PCR with oGQ1801/oGQ1623 } \\
\text { Digested with AgeI/XhoI }\end{array}$ & Cloned in this study \\
\hline $\begin{array}{l}\text { pGADT7 tbc-8 } \\
1-328 \text { aa }\end{array}$ & $A m p^{r}$ & $\begin{array}{l}\text { PCR with oGQ1622/oGQ2353 } \\
\text { Digested with AgeI/XhoI }\end{array}$ & Cloned in this study \\
\hline $\begin{array}{l}\text { pGADT7 tbc-8 } \\
329-597 \text { aa }\end{array}$ & $A m p^{r}$ & $\begin{array}{l}\text { PCR with oGQ1801/oGQ1800 } \\
\text { Digested with AgeI/XhoI }\end{array}$ & Cloned in this study \\
\hline pGADT7 rap-1 & $A m p^{r}$ & -- & $\begin{array}{l}\text { Kindly provided by } \\
\text { Sabine König }\end{array}$ \\
\hline pGADT7 rap-2 & $A m p^{r}$ & -- & $\begin{array}{l}\text { Kindly provided by } \\
\text { Sabine König }\end{array}$ \\
\hline pGADT7 rap-3 & $A m p^{r}$ & -- & $\begin{array}{l}\text { Kindly provided by } \\
\text { Sabine König }\end{array}$ \\
\hline pGADT7 ral-1 & $A m p^{r}$ & -- & $\begin{array}{l}\text { Kindly provided by } \\
\text { Sabine König }\end{array}$ \\
\hline pGADT7 ras-1 & $A m p^{r}$ & -- & $\begin{array}{l}\text { Kindly provided by } \\
\text { Sabine König }\end{array}$ \\
\hline $\begin{array}{l}\text { pGADT7 rap-1 } \\
(G 12 \mathrm{~V})\end{array}$ & $A m p^{r}$ & -- & $\begin{array}{l}\text { Kindly provided by } \\
\text { Sabine König }\end{array}$ \\
\hline $\begin{array}{l}\text { pGADT7 rap }-2 \\
(G 12 \mathrm{~V})\end{array}$ & $A m p^{r}$ & -- & $\begin{array}{l}\text { Kindly provided by } \\
\text { Sabine König }\end{array}$ \\
\hline $\begin{array}{l}\text { pGADT7 rap-3 } \\
(G 12 \mathrm{~V})\end{array}$ & $A m p^{r}$ & -- & $\begin{array}{l}\text { Kindly provided by } \\
\text { Sabine König }\end{array}$ \\
\hline $\begin{array}{l}\text { pGADT7 ral-1 } \\
(G 26 \mathrm{~V})\end{array}$ & $A m p^{r}$ & -- & $\begin{array}{l}\text { Kindly provided by } \\
\text { Sabine König }\end{array}$ \\
\hline $\begin{array}{l}\text { pGADT7 ras-1 } \\
(G 26 \mathrm{~V})\end{array}$ & $A m p^{r}$ & -- & $\begin{array}{l}\text { Kindly provided by } \\
\text { Sabine König }\end{array}$ \\
\hline
\end{tabular}




\begin{tabular}{|c|c|c|c|}
\hline pGADT7 rund-1 & $A m p^{r}$ & $\begin{array}{l}\text { PCR with oGQ1666/oGQ1667 } \\
\text { Digested with NcoI/XhoI }\end{array}$ & Cloned in this study \\
\hline $\begin{array}{l}\text { pGADT7 rund-1 } \\
1-261 \text { aa }\end{array}$ & $A m p^{r}$ & $\begin{array}{l}\text { PCR with oGQ1666/oGQ1843 } \\
\text { Digested with NcoI/XhoI }\end{array}$ & Cloned in this study \\
\hline $\begin{array}{l}\text { pGADT7 rund-1 } \\
262-549 \text { aa }\end{array}$ & $A m p^{r}$ & $\begin{array}{l}\text { PCR with oGQ1735/oGQ1667 } \\
\text { Digested with NcoI/XhoI }\end{array}$ & Cloned in this study \\
\hline pGADT7 $c c c p-1$ & $A m p^{r}$ & $\begin{array}{l}\text { PCR with oGQ2144/oGQ2145 } \\
\text { Digested with AgeI/XhoI }\end{array}$ & Cloned in this study \\
\hline pGADT7 vps-51 & $A m p^{r}$ & -- & (Luo et al, 2011) \\
\hline pGADT7 vps-52 & $A m p^{r}$ & -- & (Luo et al, 2011) \\
\hline pGADT7 vps-53 & $A m p^{r}$ & -- & (Luo et al, 2011) \\
\hline pGADT7 vps-54 & $A m p^{r}$ & -- & (Luo et al, 2011) \\
\hline $\begin{array}{l}\text { Genes of interest } \\
\text { in injection vectors }\end{array}$ & Resistance & Cloning strategy & Source \\
\hline $\begin{array}{l}\text { pPD115.62 ptbc- } \\
8:: g f p\end{array}$ & $A m p^{r}$ & $\begin{array}{l}\text { PCR with oGQ1793/oGQ2055 } \\
\text { Digested with Pst1/AgeI }\end{array}$ & Cloned in this study \\
\hline $\begin{array}{l}\text { prab-3::tagRFP- } \\
\text { tbc- } 8\end{array}$ & $A m p^{r}$ & $\begin{array}{l}\text { Subcloned from L4440-tbc- } 8 \\
\text { Digested with AgeI/XhoI }\end{array}$ & Cloned in this study \\
\hline $\begin{array}{l}\text { prab-3::tagRFP- } \\
\text { tbc-8 }(\text { R697A })\end{array}$ & $A m p^{r}$ & $\begin{array}{l}\text { Subcloned from pGADT7-tbc- } 8 \\
(R 697 A) ; \text { Digested with AgeI/XhoI }\end{array}$ & Cloned in this study \\
\hline prab-3::yfp-tbc-8 & $A m p^{r}$ & $\begin{array}{l}\text { Subcloned from L4440-tbc- } 8 \\
\text { Digested with AgeI/XhoI }\end{array}$ & Cloned in this study \\
\hline $\begin{array}{l}\text { prab-3::mcherry- } \\
\text { rab2 }\end{array}$ & $A m p^{r}$ & -- & $\begin{array}{l}\text { Kindly provided by } \\
\text { Marija Sumakovic }\end{array}$ \\
\hline prab-3::mannsII-yfp & $A m p^{r}$ & -- & $\begin{array}{l}\text { Kindly provided by } \\
\text { Marija Sumakovic }\end{array}$ \\
\hline prab-3::gfp-2xfyve & $A m p^{r}$ & -- & $\begin{array}{l}\text { Kindly provided by } \\
\text { Marija Sumakovic }\end{array}$ \\
\hline $\begin{array}{l}\text { prab-3::mcherry- } \\
\text { apt-9 }\end{array}$ & $\mathrm{Amp}^{\mathrm{r}}$ & -- & $\begin{array}{l}\text { Kindly provided by } \\
\text { Marija Sumakovic }\end{array}$ \\
\hline $\begin{array}{l}\text { prab-3::mcherry- } \\
\text { rab-5 }(Q 78 L)\end{array}$ & $A m p^{r}$ & -- & $\begin{array}{l}\text { Kindly provided by } \\
\text { Marija Sumakovic }\end{array}$ \\
\hline $\begin{array}{l}\text { prab-3::mcherry- } \\
\text { rab-5 }\end{array}$ & $A m p^{r}$ & -- & $\begin{array}{l}\text { Kindly provided by } \\
\text { Marija Sumakovic }\end{array}$ \\
\hline $\begin{array}{l}\text { prab-3::mcherry- } \\
\text { rab-7 }\end{array}$ & $A m p^{r}$ & -- & $\begin{array}{l}\text { Kindly provided by } \\
\text { Marija Sumakovic }\end{array}$ \\
\hline prab-3::ric-19-yfp & $A m p^{r}$ & -- & $\begin{array}{l}\text { Kindly provided by } \\
\text { Marija Sumakovic }\end{array}$ \\
\hline $\begin{array}{l}\text { punc-129::tagRFP- } \\
\text { tbc-8 }\end{array}$ & $A m p^{r}$ & $\begin{array}{l}\text { Subcloned from L4440-tbc- } 8 \\
\text { Digested with AgeI/XhoI }\end{array}$ & Cloned in this study \\
\hline $\begin{array}{l}\text { punc-129::tagRFP- } \\
\text { tbc-8 (R697A) }\end{array}$ & $A m p^{r}$ & $\begin{array}{l}\text { Subcloned from pGADT7-tbc-8 } \\
(R 697 A) ; \text { Digested with AgeI/XhoI }\end{array}$ & Cloned in this study \\
\hline pRF4-rol-6 & $A m p^{r}$ & co-injection marker, rol-6(su1006) & (Mello \& Fire, 1995) \\
\hline $\mathrm{p} t t x-3:: g f p$ & $A m p^{r}$ & $\begin{array}{l}\text { co-injection marker, } g f p \text { expression in } \\
\text { head neurons (AIY left and right } \\
\text { interneurons) }\end{array}$ & (Hobert et al, 1997) \\
\hline
\end{tabular}




\section{XV.5 Media}

\section{Luria-Bertani (LB) Medium}

$\begin{array}{ll}5 \mathrm{~g} / 1 & \text { Peptone (Roth) } \\ 2.5 \mathrm{~g} / 1 & \text { Yeast extract (Roth) } \\ 5 \mathrm{~g} / 1 & \mathrm{NaCl} \text { (Roth) } \\ 7.5 \mathrm{~g} / 1 & \text { Agar (Roth), for plates }\end{array}$

\section{Nematode Growth Medium (NGM)}

\begin{tabular}{|c|c|}
\hline $16 \mathrm{~g} / 1$ & Agar \\
\hline $3 \mathrm{~g} / 1$ & Peptone \\
\hline $3 \mathrm{~g} / 1$ & $\mathrm{NaCl}$ \\
\hline $25 \mathrm{ml} / 1$ & $1 \mathrm{M} \mathrm{KPO}_{4}$ Solution (Roth) \\
\hline $1 \mathrm{ml} / 1$ & $1 \mathrm{M} \mathrm{MgSO}_{4}$ Solution (Roth) \\
\hline $1 \mathrm{ml} / 1$ & $1 \mathrm{M} \mathrm{CaCl}_{2}$ Solution (Roth) \\
\hline $1 \mathrm{ml} / 1$ & 10 mg/ml Nystatin (Sigma) \\
\hline $1 \mathrm{ml} / 1$ & $5 \mathrm{mg} / \mathrm{ml}$ Cholesterol (Roth) \\
\hline
\end{tabular}

\section{Complex (YAPD) yeast media}

$\begin{array}{ll}10 \mathrm{~g} / 1 & \text { Yeast extract } \\ 20 \mathrm{~g} / 1 & \text { Peptone } \\ 20 \mathrm{~g} / 1 & \text { Glucose (Roth) } \\ 1 \mathrm{~g} / 1 & \text { Adenine Sulfate (Roth) } \\ 20 \mathrm{~g} / 1 & \text { Select agar (Invitrogen) }\end{array}$

\section{Yeast minimal growth medium}
$6.8 \mathrm{~g} / 1$
Yeast nitrogen base (Invitrogen)
$1.0 \mathrm{~g} / 1$ amino acid mix (-leu. -trp, -his, -ura)
$20 \mathrm{~g} / 1$
Select agar, for plates
pH 6-7

After autoclaving:

\begin{tabular}{lll}
\hline $10 \mathrm{ml} / 1$ & amino acid solution & $\begin{array}{l}\text { (+his } 480 \mathrm{mg} / 200 \mathrm{ml}) \\
\text { (+ura } 480 \mathrm{mg} / 200 \mathrm{ml})\end{array}$ \\
$40 \mathrm{ml} / 1$ & $50 \%$ Glucose &
\end{tabular}




\section{XV.6 Buffers and Solutions}

$10 \%$ APS solution

$\begin{array}{ll}1 \mathrm{~g} & \text { APS (Roth) } \\ 10 \mathrm{ml} & \mathrm{ddH}_{2} \mathrm{O}\end{array}$

Blot buffer, 2 liter

$\begin{array}{ll}11.6 \mathrm{~g} & \text { Tris } \\ 5.8 \mathrm{~g} & \text { Glycine } \\ 0.74 \mathrm{~g} & \text { SDS } \\ 400 \mathrm{ml} & \text { Methanol } \\ \text { pH } 8.9 & \end{array}$

Coomassie Blue Staining buffer

$\begin{aligned} 0.25 \% & \text { Coomassie Brilliant Blue R250 (BioRad) } \\ 50 \% & \text { Methanol (Roth) } \\ 10 \% & \text { Acetic acid (Roth) }\end{aligned}$

\section{Coomassie destaining buffer}

$\begin{array}{ll}5 \% & \text { Methanol (Roth) } \\ 7.5 \% & \text { Acetic acid (Roth) }\end{array}$

\section{Egg Prep Buffer}

$\begin{array}{ll}3.5 \mathrm{ml} & \text { worm solution } \\ 0.5 \mathrm{ml} & 5 \mathrm{M} \mathrm{NaOH} \\ 1 \mathrm{ml} & \text { Bleach solution }(5 \%)\end{array}$

\section{GST cleavage buffer}

$\begin{array}{ll}50 \mathrm{mM} & \text { Tris-HCl pH } 7.0-7.3 \text { (Roth) } \\ 150 \mathrm{mM} & \text { NaCl } \\ 1 \mathrm{mM} & \text { EDTA } \\ 2 \mathrm{mM} & \text { DTT (Roth) } \\ 100 \mu \mathrm{M} & \text { Pefabloc protease inhibitor cocktail (Roche) }\end{array}$

\section{GST elution buffer}

$\begin{array}{ll}50 \mathrm{mM} & \text { Tris- } \mathrm{HCl} \mathrm{pH} 8.0 \\ 150 \mathrm{mM} & \mathrm{NaCl} \\ 20 \mathrm{mM} & \text { reduced glutathione (Roth) }\end{array}$




\section{Homogenization buffer for CoIP in C. elegans}

$\begin{array}{ll}50 \mathrm{mM} & \text { HEPES } \\ 7.5 \% & \text { Glycerol } \\ 10 \mathrm{mM} & \mathrm{NaCl} \\ 1 \mathrm{mM} & \text { EDTA } \\ 1 \mathrm{Tbl} & \text { Complete Mini protease inhibitor (EDTA-free) (Roche) }\end{array}$

\section{Lysis buffer for CoIP in HEK293 cells}

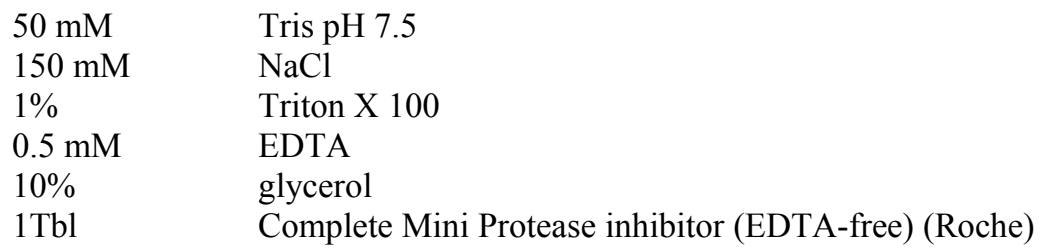

\section{M9 buffer}

$\begin{array}{ll}3 \mathrm{~g} / 1 & \mathrm{KH}_{2} \mathrm{PO}_{4} \text { (Roth) } \\ 6 \mathrm{~g} / 1 & \mathrm{Na}_{2} \mathrm{HPO}_{4} \text { (Roth) } \\ 5 \mathrm{~g} / 1 & \mathrm{NaCl} \text { (Roth) } \\ 1 \mathrm{ml} & 1 \mathrm{M} \mathrm{MgSO}_{4} \text { (Roth) }\end{array}$

PBS buffer (1x)

$\begin{array}{ll}8 \mathrm{~g} / 1 & \mathrm{NaCl} \\ 0.2 \mathrm{~g} / 1 & \mathrm{KCl} \\ 1.44 \mathrm{~g} / 1 & \mathrm{Na}_{2} \mathrm{HPO}_{4} \\ 0.24 \mathrm{~g} / 1 & \mathrm{KH}_{2} \mathrm{PO}_{4}\end{array}$

$50 \%$ PEG

$50 \mathrm{~g} \quad$ PEG 3350

Transfer $50 \mathrm{~g}$ PEG in a $150 \mathrm{ml}$ glass beaker with $35 \mathrm{ml} \mathrm{H}_{2} \mathrm{O}$ and stir until dissolving by warming up the solution for circa $30 \mathrm{~min}$. Add $\mathrm{H}_{2} \mathrm{O}$ up to $100 \mathrm{ml}$ and filter sterilize the suspension ( $0.45 \mu \mathrm{m}$ filter $)$.

10 \% Resolving Gel - 15ml

$\begin{array}{ll}5 \mathrm{ml} & 30 \% \text { Acrylamide/Bis } \\ 5.9 \mathrm{ml} & \mathrm{ddH}_{2} \mathrm{O} \\ 3.8 \mathrm{ml} & 1.5 \mathrm{M} \text { Tris, } \mathrm{pH} 8.8 \\ 150 \mu \mathrm{l} & 10 \% \text { SDS } \\ 150 \mu \mathrm{l} & 10 \% \text { APS } \\ 6 \mu \mathrm{l} & \text { TEMED }\end{array}$


Sample buffer for agarose gel loading (10x)

$\begin{array}{ll}30 \mathrm{ml} & 100 \% \text { Glycerol } \\ 20 \mathrm{ml} & \text { EDTA pH } 8.0 \\ 0.5 \mathrm{ml} & 1 \mathrm{M} \text { Tris pH 7.5 } \\ \text { A bit of } & \text { bromphenolblue } \\ \mathrm{H}_{2} \mathrm{O} \text { up to } 50 \mathrm{ml} & \end{array}$

\section{S buffer}

$\begin{array}{ll}129 \mathrm{ml} & 0.05 \mathrm{M} \mathrm{K}_{2} \mathrm{HPO}_{4} \\ 871 \mathrm{ml} & 0.05 \mathrm{M} \mathrm{KH}_{2} \mathrm{PO}_{4} \\ 5.85 \mathrm{~g} & \mathrm{NaCl} \\ \text { pH } 6.0 & \end{array}$

Autoclave or filter sterilize!

\section{SDS running buffer (10x)}

$\begin{array}{ll}30.3 \mathrm{~g} / 1 & \text { Tris } \\ 144 \mathrm{~g} / 1 & \text { Glycine (Roth) } \\ 10 \mathrm{~g} / 1 & \text { SDS (Roth) } \\ & \text { pH } 8.3\end{array}$

\section{2x SDS sample buffer}

$\begin{array}{ll}121.1 \mathrm{~g} / \mathrm{l} & \text { Tris pH } 6.8 \\ 20 \% & \text { Glycerol }(\mathrm{v} / \mathrm{v}) \\ 100 \mathrm{~g} / 1 & \text { SDS } \\ 0.002 \% & \text { Bromophenol blue }(\mathrm{w} / \mathrm{v}) \\ 200 \mathrm{ml} & \text { 2-Mercaptoethanol }\end{array}$

\section{Solutions for developing Western blots}

Solution A (store in refrigerator)

$\begin{array}{ll}200 \mathrm{ml} & 0.1 \mathrm{M} \text { Tris } \mathrm{HCl}(\mathrm{pH} 8.6) \\ 50 \mathrm{mg} & \text { Luminol (Sigma) }\end{array}$

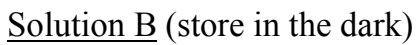
$11 \mathrm{mg}$ para-hydroxycoumarin acid (dilute in $10 \mathrm{ml}$ DMSO)
$35 \%$ $\mathrm{H}_{2} \mathrm{O}_{2}$

Procedure:

Mix $1 \mathrm{ml}$ Solution A, $100 \mu \mathrm{l}$ Solution B and $3 \mu \mathrm{l} \mathrm{H}_{2} \mathrm{O}_{2}$ (enough for one Western blot).

Distribute solution mix onto the blot and incubate for $2 \mathrm{~min}$ in the dark before imaging the blot.

\section{$5 \%$ Stacking gel $-5 \mathrm{ml}$}

$\begin{array}{ll}0.83 \mathrm{ml} & 30 \% \text { Acrylamide/Bis (Roth) } \\ 3.4 \mathrm{ml} & \mathrm{ddH}_{2} \mathrm{O} \\ 0.63 \mathrm{ml} & 1.0 \mathrm{M} \text { Tris, } \mathrm{pH} 6.8 \\ 50 \mu \mathrm{l} & 10 \% \text { SDS } \\ 50 \mu \mathrm{l} & 10 \% \text { APS (Roth) } \\ 5 \mu \mathrm{l} & \text { TEMED (Roth) }\end{array}$




\section{TBE buffer (10x)}

$\begin{array}{ll}108 \mathrm{~g} / 1 & \text { Tris } \mathrm{NaOH} \\ 55 \mathrm{~g} / 1 & \text { Boric acid (Roth) } \\ 9.3 \mathrm{~g} / 1 & \text { EDTA (pH 8.0) }\end{array}$

TE buffer

$\begin{array}{ll}10 \mathrm{mM} & \text { Tris-HCl } \mathrm{pH} 8.0 \\ 1 \mathrm{mM} & \text { EDTA (Roth) }\end{array}$

Transformation and Storage Solution for chemical transformation (TSS)

$\begin{array}{ll}85 \% & \text { LB medium }(42.5 \mathrm{ml}) \\ 10 \% & \text { PEG (wt/vol, MW 8000) }(5 \mathrm{~g}) \\ 5 \% & \text { DMSO (vol/vol) }(2.5 \mathrm{ml}) \\ 50 \mathrm{mM} & \mathrm{MgCl}_{2}(\mathrm{pH} 6.5)(0.51 \mathrm{~g})\end{array}$

Autoclave or filter sterilize! Store at $4^{\circ} \mathrm{C}$. Always prepare a fresh solution before preparing competent cells!

\section{Washing buffer for CoIP in HEK293 cells}

$\begin{array}{ll}50 \mathrm{mM} & \text { Tris pH 7.5 } \\ 500 \mathrm{mM} & \text { NaCl } \\ 0.1 \% & \text { Triton X 100 } \\ 0.5 \mathrm{mM} & \text { EDTA } \\ 10 \% & \text { glycerol } \\ 1 \mathrm{Tbl} & \text { Complete Mini Protease inhibitor (EDTA-free) (Roche) }\end{array}$

\section{Worm freezing solution}

S buffer $+30 \%$ glycerol

\section{Worm lysis/PCR buffer (1x)}

$\begin{array}{ll}100 \mathrm{mM} & \text { Tris pH 8.3 } \\ 500 \mathrm{mM} & \mathrm{KCl} \text { (Roth) } \\ 20 \mathrm{mM} & \mathrm{MgCl}_{2} \text { (Roth) } \\ 20 \mathrm{mg} / \mathrm{ml} & \text { Proteinase K added fresh prior to lysis (Roth) }\end{array}$

\section{Worm lysis/PCR buffer (10x)}

$\begin{array}{ll}1 \mathrm{M} & \text { Tris pH } 8.3 \\ 5 \mathrm{M} & \mathrm{KCl} \\ 200 \mathrm{mM} & \mathrm{MgCl}_{2}\end{array}$

Yeast transformation one-step buffer
$0.2 \mathrm{M}$
Lithium Acetate (0.4g) (Roth)
$40 \%$
PEG 4000 (12-15g) (Sigma)
$100 \mathrm{mM}$
DTT $(0.46 \mathrm{~g})$

Fill up to $30 \mathrm{ml}$ with $\mathrm{ddH}_{2} \mathrm{O}$ and filtrate 
Amino acid 100x solutions

\begin{tabular}{cl}
{$[100 \mathrm{x}] \mathrm{in} \mathrm{mg} / \mathrm{ml}$} & Component \\
\hline 4.8 & Tryptophane \\
2.4 & Histidine \\
7.2 & Leucine \\
2.0 & Uracil
\end{tabular}

Amino acid mix minus 4 (without uracil, leucine, tryptophan, histidine)

\begin{tabular}{cl} 
Mass in $g$ & Amino acid \\
\hline 2 & adenine \\
2 & L-alanine \\
2 & L-arginine \\
2 & L-asparagine \\
2 & L-aspartic acid \\
2 & L-cysteine \\
2 & L-glutamine \\
2 & L-glutamic acid \\
2 & L-glycine \\
2 & L-isoleucine \\
2 & L-lysine \\
2 & L-methionine \\
2 & L-phenylalanine \\
2 & L-proline \\
2 & L-serine \\
2 & L-threonine \\
2 & L-tyrosine \\
2 & L-valine \\
0.2 & Para- \\
& aminobenzoic \\
& acid
\end{tabular}

Pestle mix and store in the dark at RT

Table XV.11. Antibiotic concentrations used in this study

\begin{tabular}{|c|c|}
\hline $\begin{array}{c}\text { Working } \\
\text { concentration }\end{array}$ & Antibiotic \\
\hline $100 \mu \mathrm{g} / \mathrm{ml}$ & Ampicillin \\
$15 \mu \mathrm{g} / \mathrm{ml}$ & Chloramphenicol \\
$25 \mu \mathrm{g} / \mathrm{ml}$ & Kanamycin \\
\hline
\end{tabular}




\section{XV.7 Supplementary Figures}

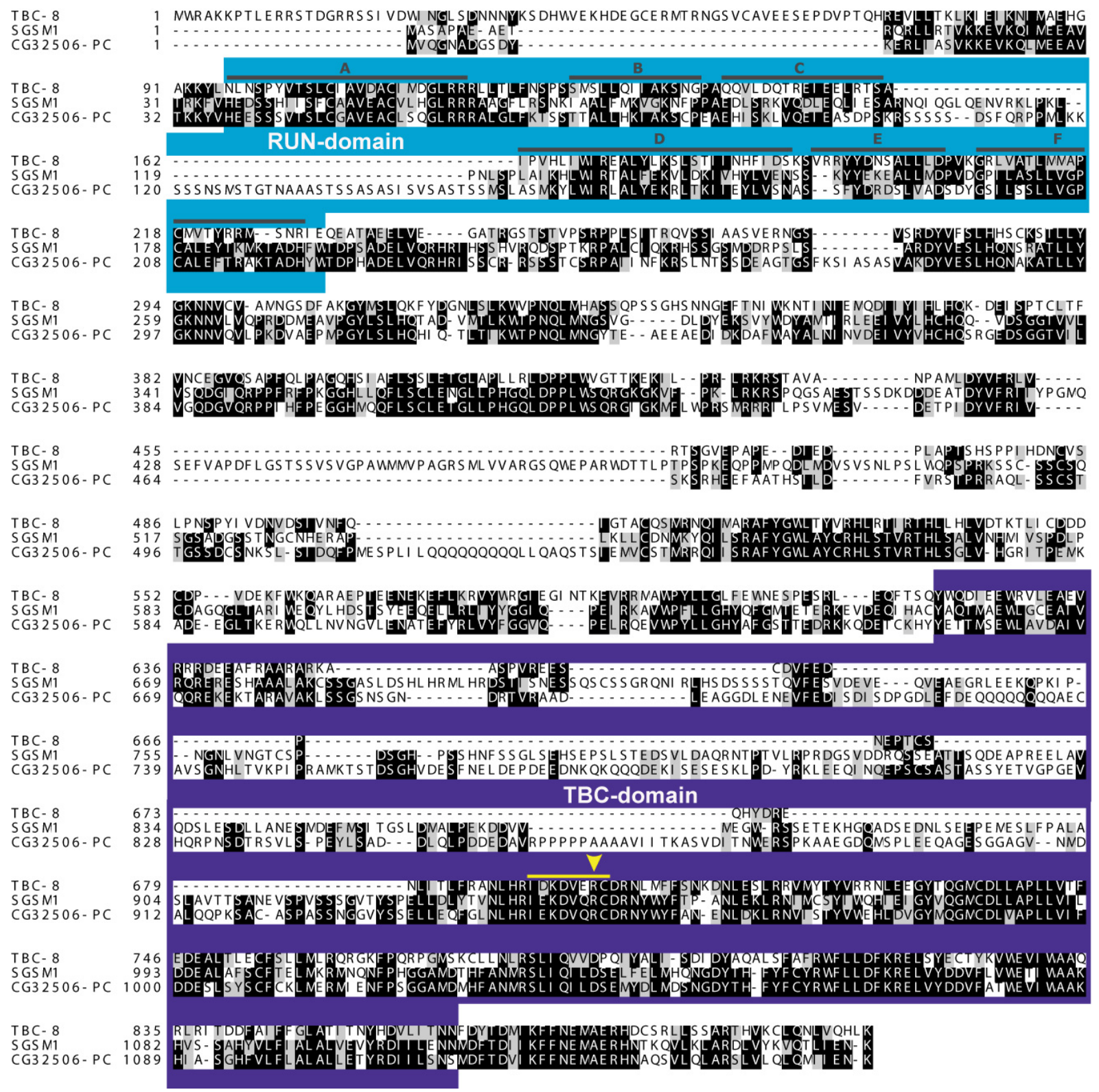

Figure XV.1. Protein sequence alignment of TBC-8 with its orthologs SGSM1 (H. sapiens) and CG32506-PC (D. melanogaster). Multiple sequence alignment was performed using the program MUSCLE and displayed using the program BOXSHADE. The predicted domains are color-coded. The RUN domain is shown in blue and the conserved blocks forming the 'core' of the RUN domain (A-F) are highlighted in black lines (Callebaut et al, 2001). The TBC-domain (prediction made by the SMART program) is depicted in purple. The catalytic arginine residue, R697, is marked by a yellow arrowhead within its catalytic motif (yellow line). Note: domain lengths predicted for TBC-8 are shown. Accession number of SGSM1: NP_001035037; FlyBase ID of CG32506-PC: FBpp0300194. Source: (Hannemann et al, 2012). 


\section{yeast two-hybrid}
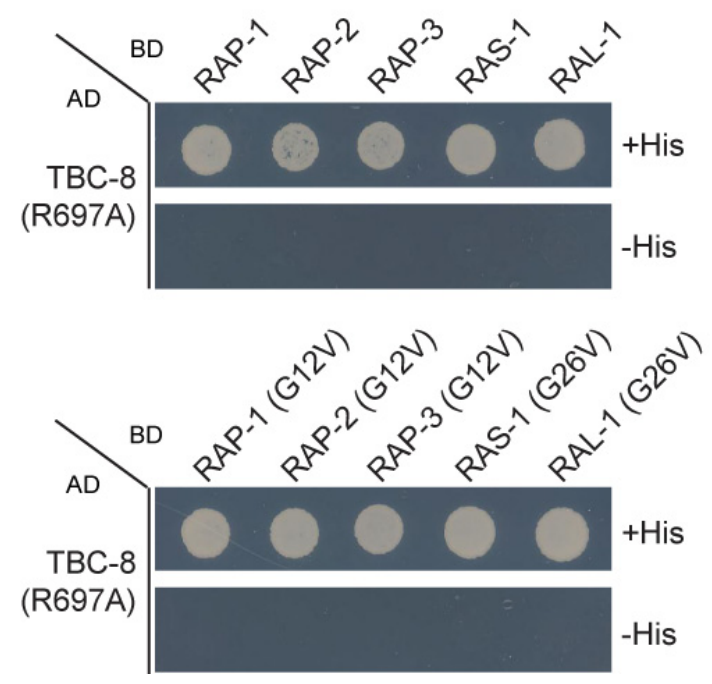

Figure XV.2. TBC-8 does not interact with RAP proteins in a yeast two-hybrid analysis. All three C. elegans RAP proteins, RAS-1 and RAL-1 in their native (upper panel) and their predicted activated state (lower panel) (Chen et al; Frische et al, 2007; Lundquist, 2006) were tested for interaction with TBC-8(R697A) in a yeast two-hybrid analysis. No growth on histidine-lacking plates was observed after 3 to 4 days. AD: Gal4p DNA activation domain fusion, BD: Gal4p DNA binding domain fusion, His: histidine. Source: (Hannemann et al, 2012). 


\section{CURRICULUM VITAE}

$\begin{array}{ll}\text { Name } & \text { Mandy Hannemann } \\ \text { Date of Birth } & \text { 12 November 1978 } \\ \text { Place of Birth } & \text { Lauchhammer } \\ \text { Address } & \text { Christophorusweg 12 } \\ & \text { 37075 Göttingen } \\ \text { Nationality } & \text { German } \\ \text { Email } & \text { mhannem@gwdg.de }\end{array}$

EDUCATION

04/2009 to date PhD Thesis

European Neuroscience Institute, Göttingen, Germany

09/2007 to 03/2009 Master of Science in Molecular Biology

$\mathrm{MSc} / \mathrm{PhD}$ Program in Molecular Biology

International Max Planck Research School

Georg-August-University

09/2003 to 02/2007 Bachelor of Science in Biotechnology

Lausitz University of Applied Sciences, Senftenberg, Germany

10/1998 to 09/2001 Professional Training as a Nurse

Carl-Thiem-Klinikum, Cottbus, Germany

08/1991 to 07/1998 Abitur

Gymnasium Senftenberg, Germany

WORK EXPERIENCE

04/2009 to date PhD Thesis

European Neuroscience Institute, Göttingen, Germany

10/2008 to 03/2009 Master Thesis

European Neuroscience Institute, Göttingen, Germany

04/2007 to 08/2007 Graduate Assistant

Lausitz University of Applied Sciences, Senftenberg, Germany

08/2006 to 01/2007 Bachelor Thesis

McMaster University, Hamilton, Canada

08/2005 to 01/2006 Practical Semester

Queensland Institute of Medical Research, Brisbane, Australia

10/2001 to 05/2003 Nurse in a Medical Department

Kantonsspital Aarau, Aarau, Switzerland 


\section{CONFERENCES ATTENDED}

May 2010

"Prof. Alvin M. Kaye student-hosted meetings: Intracellular trafficking processes”, Rehovot, Israel

Talk: "Regulation of Dense Core Vesicle maturation at the Golgi-endosomal interface in C. elegans"

May 2010

International Symposium "Protein trafficking in health and disease”, Hamburg, Germany

Talk: "Regulation of Dense Core Vesicle maturation at the Golgi-endosomal interface in C. elegans”

June 2011 18 ${ }^{\text {th }}$ International C. elegans Meeting, University of California, Los Angeles

Poster: "Regulation of the RAB-2 GTPase involved in dense core vesicle maturation"

\section{AWARDS}

June $2011 \quad$ Poster prize: "Regulation of the RAB-2 GTPase involved in dense core vesicle maturation" at $18^{\text {th }}$ International C. elegans Meeting, University of California, Los Angeles

\section{SCHOLARSHIPS}

$2007-2008$

$10 / 2007$ to date

$08 / 2006-12 / 2006$

09/2005-02/2006
International Max Planck Research School support

Scholarship from e-fellows

Scholarship from the German Academic Exchange Service (DAAD)

Scholarship from the Internationale Weiterbildung und Entwicklung gGmbH (InWEnt)

\section{LANGUAGES}

German native speaker English fluent (written and spoken)

Russian basic knowledge

\section{FURTHER ACTIVITIES}

Member of the Organization Team of the $7^{\text {th }} \mathrm{PhD}$ Symposium "Horizon in Molecular Biology” 2010

Member of the Organization Team of the $6^{\text {th }} \mathrm{PhD}$ Symposium "Horizon in Molecular Biology” 2009 


\section{List of Publications}

Sasidharan N, Sumakovic M, Hannemann M, Hegermann J, Liewald J, Olendrowitz C, Koenig S, Grant B, Gottschalk A, Eimer S. Rab5 and Rab10 cooperate to regulate neuropeptide release in Caenorhabditis elegans. PNAS. Manuscript in Revision.

Ailion M, Hannemann M, Dalton S, Pappas A, Watanabe S, Hegermann J, Han HF, Gu M, Schuske K, Hullett P, Eimer S, Jorgensen EM. A Rab2 effector complex mediates dense-core vesicle maturation. Neuron. Manuscript in Revision.

Hannemann M, Sasidharan N, Hegermann J, Kutscher LM, Koenig S, Eimer S. 2012. TBC-8, a Putative RAB-2 GAP, Regulates Dense Core Vesicle Maturation in Caenorhabditis elegans. PLoS Genet. 8: e10027222.

Luo L, Hannemann M, Koenig S, Hegermann J, Ailion M, Cho MK, Sasidharan N, Zweckstetter M, Rensing SA, Eimer S. 2011. The Caenorhabditis elegans GARP complex contains the conserved Vps51 subunit and is required to maintain lysosomal morphology. Mol Biol Cell. 22(14):2564-78.

Cooper CA, Zhang K, Andres SN, Fang Y, Kaniuk NA, Hannemann M, Brumell JH, Foster LJ, Junop MS, Coombes BK. 2010. Structural and biochemical characterization of SrcA, a multi-cargo type III secretion chaperone in Salmonella required for pathogenic association with a host. PLoS Pathog. 6(2):e1000751.

Spielmann T, Dixon MW, Hernandez-Valladares M, Hannemann M, Trenholme KR, Gardiner DL. 2006. Reliable transfection of Plasmodium falciparum using noncommercial plasmid mini preparations. Int J Parasitol. 36(12):1245-8.

Spielmann T, Hawthorne PL, Dixon MW, Hannemann M, Klotz K, Kemp DJ, Klonis N, Tilley L, Trenholme KR, Gardiner DL. 2006. A cluster of ring stage-specific genes linked to a locus implicated in cytoadherence in Plasmodium falciparum codes for PEXEL-negative and PEXEL-positive proteins exported into the host cell. Mol Biol Cell. 17(8):3613-24. 